\title{
RAFT-Polymerisation an Oberflächen
}

\author{
Dissertation \\ zur Erlangung des Doktorgrades \\ der Mathematisch-Naturwissenschaftlichen Fakultäten \\ der Georg-August-Universität zu Göttingen
}

\author{
vorgelegt von \\ Duc Hung Nguyen \\ aus \\ Saigon / Vietnam
}

Göttingen 2007 
D 7

Referent:

Prof. Dr. M. Buback

Korreferent:

Prof. Dr. H. U. Krebs

Tag der mündlichen Prüfung:

03.07.2007 
Meiner Familie 
Auf vorbestimmten Pfaden suche ich nach Erkenntnis.

(E Nomine) 


\section{Inhaltsverzeichnis}

1 Zusammenfassung 1

2 Einleitung und Grundlagen $\quad 3$

2.1 Einführung . . . . . . . . . . . . . . . . . . . . . . 4

2.2 Lebende Polymerisation . . . . . . . . . . . . . . . . . 6

2.3 Freie radikalische Polymerisation _. . . . . . . . . . . . 7

2.3.1 Kettenstart ...................... 8

2.3 .2 Kettenwachstum . . . . . . . . . . . . . 9

2.3.3 Kettenabbruch . . . . . . . . . . . . . . 9

2.3.4 Kettenübertragung . . . . . . . . . . . . . . . 10

2.3.5 Gesamtreaktion .................. . 11

2.3.6 Polymerisationsgrad . . . . . . . . . . . . . . 11

2.4 Kontrollierte radikalische Polymerisation . . . . . . . . . . . . . . 12

2.4.1 Allgemeines Prinzip . . . . . . . . . . . . . . . 12

2.4.2 Kombination und Homolyse : NMP . . . . . . . . . . . 16

2.4.3 Übergangsmetall-katalysierter Atomtransfer : ATRP . . . . . . 18

2.4.4 Degenerative Kettenübertragung : RAFT . . . . . . . . . . . . 19

2.4.5 Vergleich der kontrollierten Polymerisationsmethoden . . . . . 20

2.5 Grundlagen der RAFT-Polymerisation . . . . . . . . . . . . . . . . . 22

2.5.1 Kinetik der RAFT-Polymerisation . . . . . . . . . . . . . . 22

2.5.2 Retardierung der Polymerisationsgeschwindigkeit . . . . . . 24

2.5.3 Das RAFT-Agens und das Design seiner chemischen Struktur 27

2.6 Motivation und Zielsetzung . . . . . . . . . . . . . . . 29

2.6.1 Heterogene Polymerisations-Katalyse . . . . . . . . . . . 30

2.6.2 Oberflächenmodifizierung . . . . . . . . . . . 32 
2.6.3 Weitere Motivationen . . . . . . . . . . . . . . . 34

Teil A: Heterogene RAFT-Polymerisation 35

3 An Silica gebundenes Dithiobenzoat $\quad 37$

3.1 Einführung . . . . . . . . . . . . . . . 37

3.2 Experiment . . . . . . . . . . . . . . . . . . 43

3.2.1 Synthese von gebundenem Cumyldithiobenzoat . . . . . . . 43

3.2.2 Polymerisation . . . . . . . . . . . . . . . 46

3.2.3 Abspaltung des gebundenen Polymers . . . . . . . . . . . . . . 47

3.3 Ergebnisse und Diskussion . . . . . . . . . . . . . . . 47

3.3.1 Charakterisierung von Silica-CDB . . . . . . . . . . 47

3.3.2 Simulation der RAFT-Polymerisation . . . . . . . . . . . . . 48

3.3.3 Einfluss der Partikelkonzentration und der Belegungsdichte des RAFT-Agens . . . . . . . . . . . . . 50

3.3.4 Molmassen-Entwicklung des toten Polymers mit dem Monomerumsatz . . . . . . . . . . . . . . 53

3.3.5 Abspaltung und Analyse des lebenden Polymers . . . . . . . . 55

3.3.6 An Silica gebundene Dithiobenzoesäure . . . . . . . . . . . 58

3.4 Schlussfolgerung . . . . . . . . . . . . . . . . . . . . . . . . . . 61

4 An Silica gebundenes Trithiocarbonat 63

4.1 Einführung . . . . . . . . . . . . . . . . . . 63

4.2 Experiment . . . . . . . . . . . . . . . . 64

4.2.1 Synthese von an Silica gebundenem Trithiocarbonat . . . . . . 64

4.2.2 Polymerisation . . . . . . . . . . . . . . 67

4.2 .3 Blockcopolymerisation . . . . . . . . . . . . . 67

4.2.4 Abspaltung des an Silica gebundenen Polymers . . . . . . . . 68

4.3 Ergebnisse und Diskussion . . . . . . . . . . . . . . . . 69

4.3.1 Die chemische Verankerung über Trimethoxysilan . . . . . . . 69

4.3.2 Homogene RAFT-Polymerisation . . . . . . . . . . . . 70

4.3.3 Heterogene RAFT-Polymerisation . . . . . . . . . . . . . . . . 71

4.3.4 Kombination von homogener und heterogener RAFT-Polymerisation . . . . . . . . . . . . . 75 
4.3.5 Charakterisierung . . . . . . . . . . . . . 78

4.3.6 Glasübergangstemperatur . . . . . . . . . . . . 82

4.3.7 Synthese des gebundenen Blockcopolymers . . . . . . . . . . 86

4.4 Schlußfolgerung . . . . . . . . . . . . . . . . . . . . . 88

5 An Wang-Harz gebundenes Xanthat $\quad 91$

5.1 Einführung . . . . . . . . . . . . . . . . . . . . . . . 91

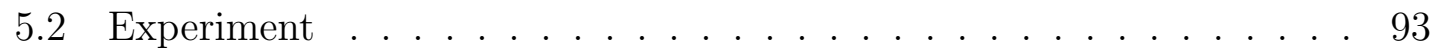

5.2.1 Synthese von an Wang-Harz gebundenem Xanthat . . . . . . . 93

5.2 .2 Polymerisation . . . . . . . . . . . . . . 96

5.2.3 Abspaltung des gebundenen Polymers . . . . . . . . . . . 97

5.3 Ergebnisse und Diskussion . . . . . . . . . . . . . . . . 97

5.3.1 Lösungsmitteleinfluss . . . . . . . . . . . . . . . . . . . 97

5.3.2 Einfluß der Partikelkonzentration von Wang-Harz . . . . . . . 100

5.3.3 Bestimmungsmethoden für den Monomermsatz . . . . . . . . 102

5.3.4 Abspaltung des gebundenen Polyvinylacetats . . . . . . . . . . 105

5.3.5 Einfluss von zusätzlichem freiem Xanthat . . . . . . . . . . . 108

5.3.6 Direkte Charakterisierung des gebundenen Polyvinylacetats . . 112

5.4 Schlussfolgerung . . . . . . . . . . . . . . . . . . . . 117

Teil B: Homogene RAFT-Polymerisation $\quad 119$

6 Polymerisation mit Dithiobenzoesäure 121

6.1 Einführung . . . . . . . . . . . . . . . . . . . . 121

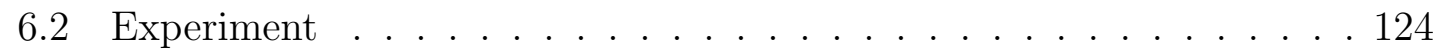

6.2.1 Chemikalien . . . . . . . . . . . . . . . . 124

6.2.2 Polymerisation . . . . . . . . . . . . . . . 125

6.3 Ergebnisse und Diskussion . . . . . . . . . . . . . . . 126

6.3.1 Induktion und Retardierung . . . . . . . . . . . . 126

6.3.2 Kontrollierte radikalische Polymerisation . . . . . . . . . . 129

6.3.3 Effektive RAFT-Agens-Konzentration . . . . . . . . . . . . 131

6.3.4 Endgruppenanalyse . . . . . . . . . . . . . . . 133

6.3.5 Vorgeschlagener Mechanismus in der Induktionsphase . . . . . 141

6.3.6 Modellierung . . . . . . . . . . . . . . . . . . . . . 144 
6.3 .7 Initiator-Cocktail . . . . . . . . . . . . . . . . . 150

6.3.8 Retardierungseffekt . . . . . . . . . . . . . . 152

6.4 Schlussfolgerung . . . . . . . . . . . . . . . . . . . . 154

7 Block-, Stern-, Kamm- und Netzwerk-Polymere 157

7.1 Einführung . . . . . . . . . . . . . . . . 157

7.2 Experiment . . . . . . . . . . . . . . . . 158

7.2.1 Synthese von RAFT-Agenzien . . . . . . . . . . . . . . 158

7.2 .2 Polymerisation . . . . . . . . . . . . . 166

7.2.3 Blockcopolymerisation . . . . . . . . . . . 167

7.2 .4 Kondensation . . . . . . . . . . . . . . . 167

7.3 Ergebnisse und Diskussion . . . . . . . . . . . . . . . 167

7.3.1 Homopolymer . . . . . . . . . . . . . . . . . 167

7.3 .2 Copolymer . . . . . . . . . . . . . . . . . . 172

7.3.3 Unsterbliche RAFT-Polymerisation . . . . . . . . . . . . . 178

7.3.4 Stern- und Kammpolymere . . . . . . . . . . . . . . . 180

7.3.5 Polymernetzwerk . . . . . . . . . . . . . . . . 187

7.3.6 Immobilisierung des Polymers über "grafting-to“ . . . . . . . . 189

7.4 Schlussfolgerung . . . . . . . . . . . . . . . . . . . . . . . . 189

8 Ausblick 191

$\begin{array}{ll}\text { Anhang } & 193\end{array}$

A Experimenteller Teil 193

A.1 Chemikalien . . . . . . . . . . . . . . . . . . . 193

A.1.1 Substrate . . . . . . . . . . . . . . . . 193

A.1.2 RAFT-Agens . . . . . . . . . . . . . . 195

A.1.3 Monomere . . . . . . . . . . . . . . . . . 195

A.1.4 Initiator . . . . . . . . . . . . . . . . . . 195

A.2 Charakterisierung und Analytik . . . . . . . . . . . . 195

A.2.1 GPC-Analyse . . . . . . . . . . . . . . 195

A.2.2 FTIR-Spektroskopie . . . . . . . . . . . . . . . . . . 197

A.2.3 Raman-Spektroskopie . . . . . . . . . . . . . . . . . . 197 
A.2.4 NMR-Spektroskopie . . . . . . . . . . . . . . . 198

A.2.5 Massenspektrometrie . . . . . . . . . . . . . . 198

A.2.6 Thermische Analyse . . . . . . . . . . . . . . . . . . . 198

A.2.7 Elementaranalyse . . . . . . . . . . . . . . . . 199

A.3 Simulation . . . . . . . . . . . . . . . . . . . . . . 199

$\begin{array}{ll}\text { Verzeichnis der Abkürzungen } & 201\end{array}$

$\begin{array}{ll}\text { Literaturverzeichnis } & 203\end{array}$

$\begin{array}{ll}\text { Danksagung } & 215\end{array}$

$\begin{array}{ll}\text { Lebenslauf } & 217\end{array}$ 


\section{Kapitel 1}

\section{Zusammenfassung}

Im Mittelpunkt dieser Arbeit steht die heterogene RAFT-Polymerisation an festen Oberflächen. Dabei verfolgt die Arbeit zwei Hauptziele, die durch eine Immobilisierung des RAFT-Agens über dessen stabilisierende Z-Gruppe gleichzeitig erreicht werden konnten. Zum einen wird ein heterogenes Katalysatorsystem für die RAFT-Polymerisation entwickelt, wodurch das wertvolle RAFT-Agens nach der Polymerisation abgetrennt und wiedergewonnen werden kann. Zum anderen wird die Oberfläche eines festen Substrats mit einem Polymer modifiziert, das durch die kontrollierte Polymerisationstechnik eine wohldefinierte Architektur besitzt.

Mit der vorliegenden Arbeit ist es gelungen, heterogene RAFT-Polymerisationen mit gebundenen Dithiobenzoaten (Kapitel 3), mit Trithiocarbonaten (Kapitel 4) und mit Xanthaten (Kapitel 5) erfolgreich durchzuführen. Als Substrat wurden sowohl Silica-Nanopartikel als auch vernetzte polymere Trägermaterialien eingesetzt.

Die Immobilisierung des RAFT-Agens über die Z-Gruppe erlaubt die Herstellung der wohldefinierten gebundenen Polymerketten von bis zu $15 \mathrm{~kg} / \mathrm{mol}$. Das gebundene Polymer (Polystyrol, Polymethylmethacrylat, Polymethylacrylat, Polyvinylacetat und Blockcopolymer PMA-b-PSty) konnte für die Charakterisierung vom festen Substrat abgespalten werden. Darüber hinaus ist es dadurch gelungen, das immobilisierte RAFT-Agens von dem polymeren Material zu trennen und wiederzugewinnen.

Zur effizienten Modifizierung der Oberflächen von festen Substraten mit Polymeren wurde die Möglichkeit erforscht, zusätzlich zu dem gebundenen RAFT-Agens der Polymerisationsmischung ein freies RAFT-Agens zuzusetzen, um eine engere und definiertere Molmassenverteilung des immobilisierten Polymers zu erhalten. 
Die modifizierten Silica-Nanopartikel, die durch die heterogene RAFT-Polymerisation Polymerketten an der Oberfläche tragen, sind bezüglich deren Eigenschaften als Füllstoffe für Polymermaterialien untersucht worden. Es hat sich dabei gezeigt, dass die für die Bearbeitung und Anwendung wichtige Glastemperatur des Materials durch Zugabe der modifizierten Silicapartikel variiert werden konnte.

Einen Beitrag zur Aufklärung der komplexen Kinetik der kontrollierten Polymerisation mit Dithiobenzoesäure konnte die Kombination aus Experiment und Computersimulationen im Rahmen dieser Arbeit liefern (Kapitel 6). Dabei wurde ein detaillierter Mechanismus für die in situ Umwandlung von Dithiobenzoesäure in ein RAFT-Agens vorgeschlagen. Auf der Grundlage dieses Mechanismus wurde ein Protokoll für die Verwendung eines Initiator-Cocktails entwickelt, um die Induktionszeit zu verkürzen und um die Menge des in situ gebildeten RAFT-Agens deutlich zu erhöhen. Dieses Protokoll stellt eine attraktive Alternative zur klassischen RAFTPolymerisation dar, weil die Dithiobenzoesäure viel einfacher und kostengünstiger zu synthetisieren ist als konventionelle RAFT-Agenzien.

Außerdem wurden im Rahmen dieser Arbeit die neuentwickelten RAFT-Agenzien in Hinblick auf deren Verwendung zur Bildung neuartiger Polymere getestet (Kapitel 7). Unterschiedliche Strategien zur Herstellung statistischer Copolymere sowie Di- und Triblockcopolymere sind mit Hilfe der neu synthetisierten RAFTAgenzien erprobt worden. So konnte durch eine Kombination von RAFT-Polymerisation und konventioneller Polymerisation mit einmaliger Transferreaktion zu einem thiol-haltigen RAFT-Agens der Einbau von Polymerblöcken ermöglicht werden, die durch die herkömmliche RAFT-Technik nicht zugänglich sind. Um die Bildung des toten Polymers zu unterdrücken, wurde das Konzept der sogenannten „unsterblichen“ RAFT-Polymerisation erfolgreich entwickelt, bei der bifunktionelle RAFT-Agenzien zum Einsatz kommen. Verschiedene polymere Architekturen wie Stern, Kamm und Netzwerk konnten durch die Kondensation von vorgefertigten reaktiven Polymeren synthetisiert werden.

Mit den vorgestellten Ergebnissen konnte eine Brücke, ausgehend von Homopolymeren über Block-, Stern-, Kamm- und Netzwerk-Polymere bis hin zu gebundenen Polymeren an festen Oberflächen durch kontrollierte RAFT-Polymerisation geschlagen werden. 


\section{Kapitel 2}

\section{Einleitung und Grundlagen}

Die Natur ist ein Meister der Polymerisation. Jedes Jahr produziert (und zerstört!) die Natur ca. 300·10 ${ }^{9}$ Tonnen Biopolymer, davon $95 \%$ in Pflanzen und $5 \%$ in Tieren [1]. Zum Vergleich beträgt die Weltproduktion aller synthetischen Polymere im gleichen Zeitraum im Schnitt nur ca. $0.23 \cdot 10^{9}$ Tonnen. Für den menschlichen Verbrauch werden jährlich ca. $7 \cdot 10^{9}$ Tonnen Biomasse in Form von Holz und Nahrung entnommen. Im Prinzip könnte man für die menschliche Versorgung auf einen beträchtlichen Teil des ungenutzten Restes von 293·10 Tonnen Biomasse zurückgreifen und so den ökologisch bedenklichen Verbrauch der fossilen Rohstoffe Erdöl, Erdgas und Kohle einschränken ${ }^{1}$. Tatsächlich ist das aus landwirtschaftlichen, politischen, chemischen und nicht zuletzt wirtschaftlichen Gründen nicht möglich. Nachwachsende Rohstoffe sind vorteilhaft, wenn sie direkt zu verbrauchernahen Produkten verarbeitet werden. Die Eigenschaften solcher Produkte aus Biopolymeren können aber nur in einem engen Bereich variiert werden. Vollsynthetische Polymere können dagegen sozusagen „nach Maß“ für den vorgesehenen Verwendungszweck hergestellt werden. Damit schlug die Geburtsstunde der vollsynthetischen Kunststoffe zu Beginn des letzten Jahrhunderts. Im Jahr 2006 erwirtschaftete die in Deutschland hoch entwickelten Kunststoff-Industrie mit einem Umsatz von etwa 80 Milliarden Euro knapp $7 \%$ des Bruttoinlandsprodukts [3].

\footnotetext{
${ }^{1}$ Die chemische Industrie verbraucht etwa $9 \%$ der gesamten Förderung von fossilen Brennstoffen, wovon $5 \%$ in die Kunststoffherstellung gehen [2].
} 


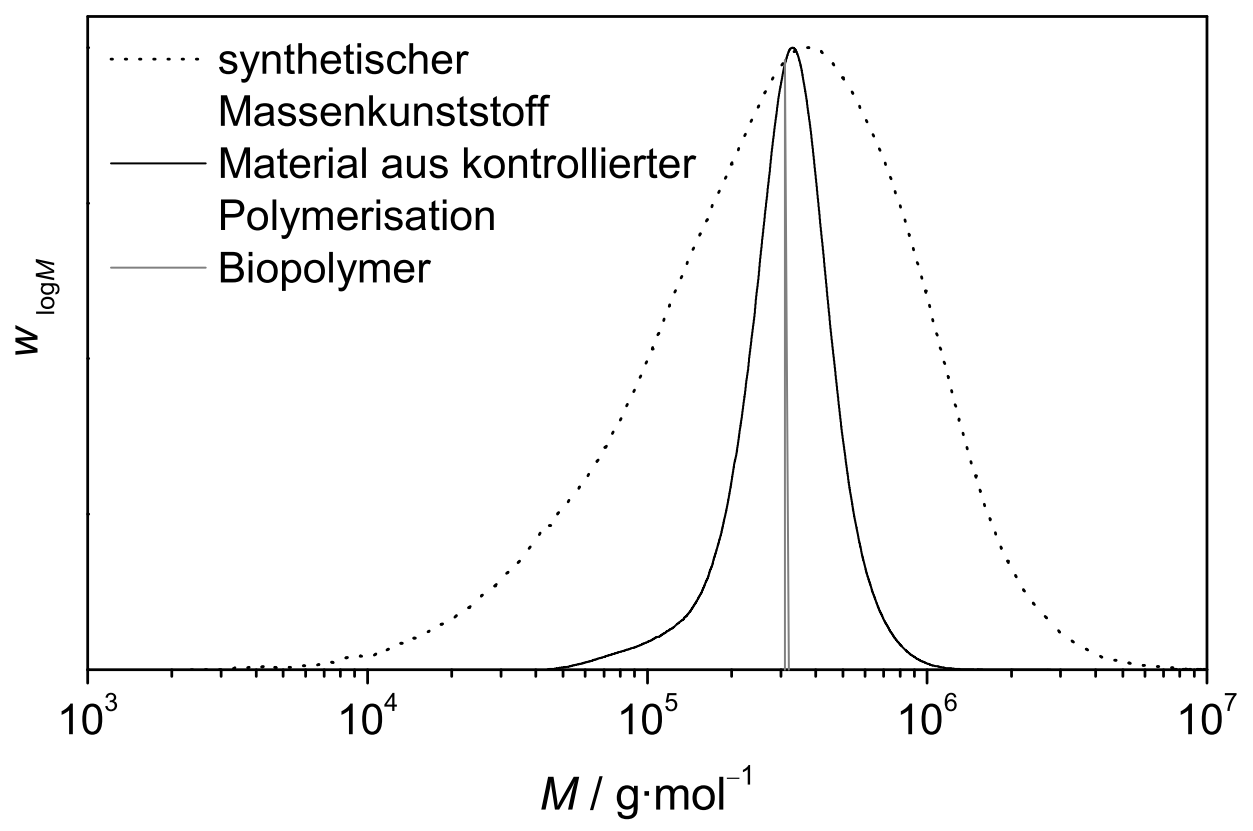

Abbildung 2.1: Schematische Darstellung der Molekulargewichtsverteilungen eines synthetischen Massenkunststoffes und eines Polymers aus einem kontrollierten radikalischen Polymerisationsprozess im Vergleich mit der definierten Molmasse eines Proteins.

\section{$2.1 \quad$ Einführung}

Unter den zahlreichen Polyreaktionen zur Herstellung von Kunststoffen erlauben besonders die kontrollierten Polymerisationen einen gezielten Aufbau von Strukturen und eine exakte Kontrolle der molekularen Architektur. Mit ihnen ist es z. B. möglich, enge Molekulargewichtsverteilungen zu erhalten, wie man in Abbildung 2.1 erkennen kann. Synthetische Polymere haben keine einheitliche Molmasse, sie besitzen eine Molekulargewichtsverteilung, die mehr oder weniger breit ist. Synthetische Massenkunststoffe wie Polyethylen, Polystyrol oder Polyvinylchlorid haben eine sehr breite Verteilung, während die Materialien aus kontrollierter Polymerisation relativ enge Molmassenverteilungen aufweisen. Biopolymere wie Enzyme oder Proteine haben eine exakte Molmasse und eine definierte dreidimensionale Struktur. Allein bei Betrachtung der Molekulargewichtsverteilung muss man wieder respektvoll anerkennen, dass die Natur nicht nur der größte Polymerhersteller ist, sondern auch ein Meister der Konstruktion von makromolekularem Material ist. 


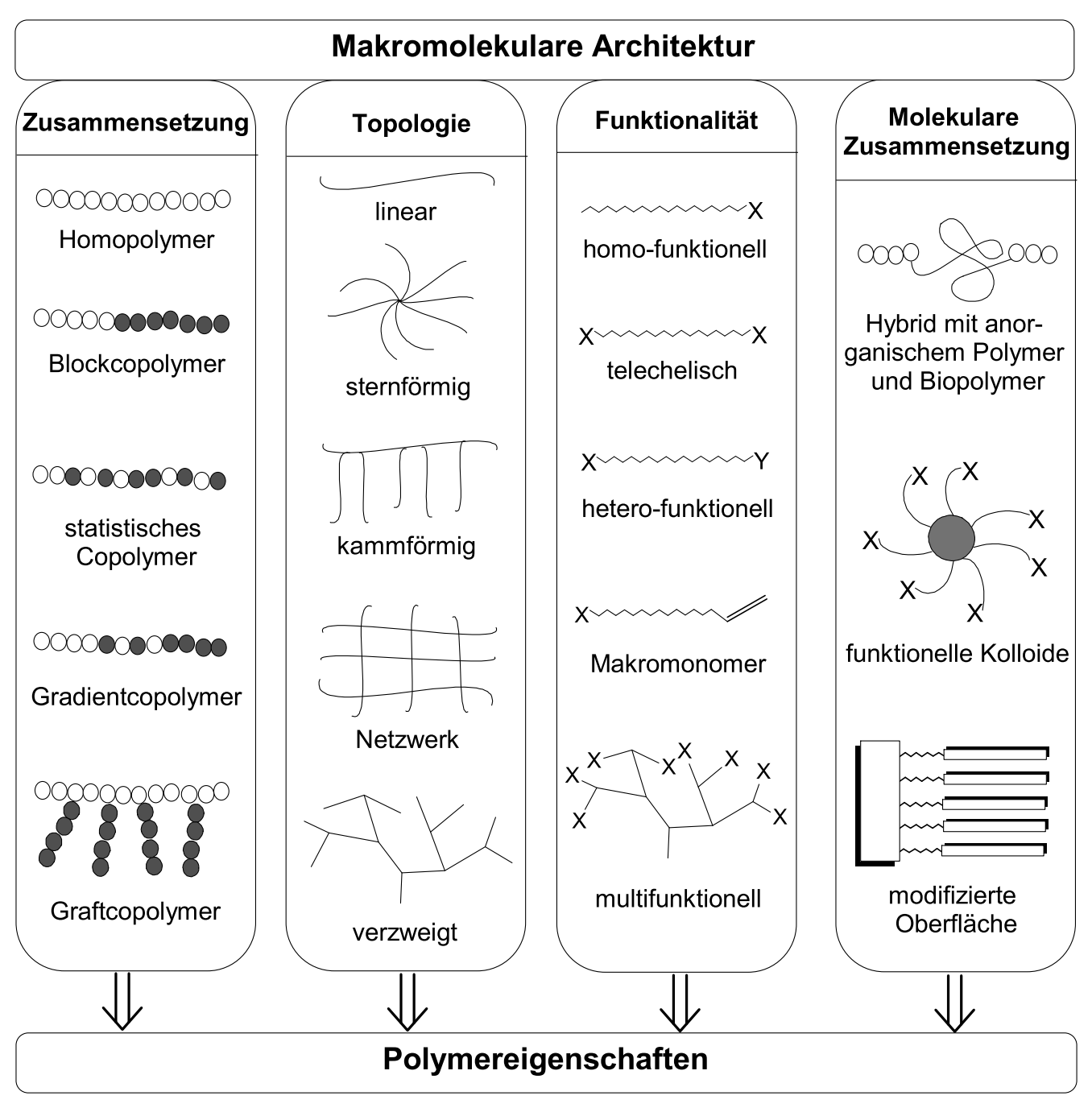

Abbildung 2.2: Molekulare Architekturen, die durch kontrollierte radikalische Polymerisation zugänglich sind [4].

Mit der Natur als Vorbild richteten wir nun unser Interesse auf die Entwicklung leistungsfähiger, gut funktionierender und selektiver Polymerisationen für die effiziente Synthese neuartiger nützlicher Materialen. Um diese Ziele zu erreichen, sind sowohl eine genaue Kenntnis der Struktur-Eigenschaftsbeziehungen notwendig als auch Polymerisationsverfahren, die eine exakte Kontrolle der molekularen Architektur eines Polymers ermöglichen.

In den letzten Jahren sind eine Reihe neuer Polymerisationsverfahren entwickelt worden, die es ermöglichen, definierte molekulare Strukturen aufzubauen. Intensive 
Untersuchungen und eine Vielzahl von Veröffentlichungen zeigen, dass verschiedene Verfahren auf dem Gebiet der kontrollierten radikalischen Polymerisation in der Lage sind, eine große Zahl neuer Polymere und Polymerarchitekturen hervorzubringen.

Abbildung 2.2 zeigt eine Auswahl von molekularen Architekturen, die durch kontrollierte radikalische Polymerisation zugänglich sind. Beispiele sind hochverzweigte Polymere, Stern-, Kamm- und Blockcopolymere mit unterschiedlichen Zusammensetzungen und Funktionalitäten. Auch die Steuerung der Taktizität, der Einsatz neuer Comonomere, die Darstellung von Hybridmaterialien mit anorganischen Polymeren und Biopolymeren oder die gezielte Modifizierung von Oberflächen sind inzwischen möglich. Besonders die Modifizierung von Nanopartikel-Oberflächen ermöglicht es, die Eigenschaften dieser Nano-Materialien gezielt zu steuern.

In diesem Kapitel wird der aktuelle Erkenntnisstand zum Thema dieser Arbeit ohne Anspruch auf Vollständigkeit dargestellt. Hier sollen einige Bemerkungen zu den folgenden Teilen gleichsam vor die Klammer gezogen werden; deshalb skizziere ich in den folgenden Abschnitten die Entwicklung allein derjenigen Erkenntnisse, die im Weiteren von Belange sind.

\subsection{Lebende Polymerisation}

Die Synthese von neuem Polymermaterial mit wohldefinierter Zusammensetzung, Architektur und Funktionalität ist schon seit langem ein Ziel der Polymerchemie. Bereits im Jahr 1936 berichtete Ziegler von der anionischen Polymerisation von Styrol und Butadien, die bei Zugabe von Alkyllithium als Initiator ohne Kettentransfer und Terminierung verläuft [5]. Während dieser Polymerisation bleibt die Anzahl der Polymermoleküle konstant. Szwarc hat später den Begriff „lebende“ Polymerisation für die Methode eingeführt, weil die Endgruppe der Polymerkette aktiv bleibt [6]. Die allgemeinen Charakteristika der lebenden Polymerisation sind [7]:

- Alle Kettenenden wachsen mit gleicher Geschwindigkeit.

- Die Konzentration der aktiven Spezies entspricht der Zahl der Polymerketten und bleibt während der Polymerisation konstant.

- Die Molmassenverteilung ist sehr eng und hat daher eine Polydispersität nahe 
eins. Sie entspricht im Idealfall einer Poisson-Verteilung.

- Die Molmasse wächst linear mit dem Monomerumsatz an.

Diese Anforderungen wurden bislang nur von der anionischen Polymerisation und mit Einschränkungen von der kationischen und der Gruppentransfer-Polymerisation erfüllt. Jedoch ist für all diese Polymerisationsarten nachteiliger Weise ein großer präparativer Aufwand erforderlich. Da bei einer lebenden Polymerisation keine Terminierungsreaktion stattfindet, bleibt die aktive Endgruppe erhalten, auch nachdem alle Monomermoleküle verbraucht sind; und wenn frisches Monomer sequentiell zugegeben wird, setzt sich die Polymerisation fort. Außerdem kann durch die Wahl eines geeigneten Abbruchreagenzes eine Endfunktionalität eingeführt werden. Somit ist ein komfortabler und effektiver Zugang zu komplizierten Polymerarchitekturen, wie beispielsweise Block-, Kamm- oder Sternpolymeren möglich.

\subsection{Freie radikalische Polymerisation}

Bis 1991 waren akademische und industrielle Forschung im Bereich der lebenden Polymerisation vorwiegend auf anionische [8], kationische [9], koordinative [10], ringöffnende [11] und Gruppentransfer-Polymerisationen fokussiert. Ein Verfahren zur lebenden radikalischen Polymerisation konnte bis zu diesem Zeitpunkt noch nicht realisiert werden, obwohl die radikalische Polymerisation der am weiteste verbreitete Polymerisationsprozess war. Mehr als 50 \% aller kommerziellen Polymere werden mittels freier radikalischer Polymerisation hergestellt. Insbesondere Massenkunststoffe wie Polyethylen niedriger Dichte (LDPE), Polystyrol (PS), Polyvinylchlorid (PVC), Polyacrylnitril (PAN) und Polymethylmethacrylat (PMMA oder Plexiglas) werden fast ausschließlich durch freie radikalische Polymerisation hergestellt.

Die freie radikalische Polymerisation ist aufgrund der Fähigkeit, eine Vielzahl unterschiedlicher Monomere in weiten Zustandsbereichen zu polymerisieren, für industrielle Anwendungen vorteilhafter als andere Polymerisationsmethoden. Außerdem ist die radikalische Polymerisation im Gegensatz zur anionischen Polymerisation z. B. wesentlich unempfindlicher gegenüber Wasser und Verunreinigungen [4].

Die freie radikalische Polymerisation ist eine Kettenreaktion. Kettenstart (Initiierung), Kettenwachstum (Propagation) und Kettenabbruch (Terminierung) finden 
Kettenstart

$$
\mathrm{I}_{2} \underset{f}{\stackrel{k_{\mathrm{d}}}{\longrightarrow}} 2 \mathrm{r}^{\cdot} \quad \mathrm{I} \cdot \underset{\text { Monomer }}{\stackrel{k_{\mathrm{p}, \mathrm{ni}}}{\longrightarrow}} \mathrm{P}_{1}^{\cdot}
$$

Kettenwachstum

$$
P_{n}^{\cdot} \underset{\text { Monomer }}{\stackrel{k_{p}}{\longrightarrow}} P_{n+1}^{\cdot}
$$

Kettenabbruch

$$
\mathrm{P}_{\mathrm{n}}^{\cdot}+\mathrm{P}_{\mathrm{m}}^{\cdot} \stackrel{k_{\mathrm{t}, \mathrm{k}}}{\longrightarrow} \mathrm{P}_{\mathrm{n}+\mathrm{m}} \quad \mathrm{P}_{\mathrm{n}}^{\cdot}+\mathrm{P}_{\mathrm{m}}^{\cdot \stackrel{k_{\mathrm{t}, \mathrm{d}}}{\longrightarrow}} \mathrm{P}_{\mathrm{n}}+\mathrm{P}_{\mathrm{m}}
$$

Kettenübertragung

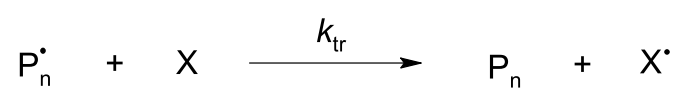

Abbildung 2.3: Kinetisches Schema der freien radikalischen Polymerisation.

während der Polymerisation parallel nebeneinander statt. In Hinblick auf die Produkteigenschaften (beispielsweise Breite und Form der Molekulargewichtsverteilung) müssen auch Kettenübertragungsreaktionen (Transfer) berücksichtigt werden. Abbildung 2.3 zeigt das kinetische Schema der freien radikalischen Polymerisation.

\subsubsection{Kettenstart}

Als Initiatoren finden Verbindungen Verwendung, die durch Zufuhr von thermischer, chemischer oder photochemischer Energie Radikale bilden, wie z. B. Azo- oder Peroxyverbindungen. Des Weiteren können auch Monomere wie Styrol bei hoher Temperatur zu Radikalbildungsreaktionen angeregt werden. Die nun reaktiven Radikale lagern im ersten Schritt an ein Monomer an und starten so die Kettenreaktion. Die Radikalbildungsgeschwindigkeit $R_{\mathrm{d}}$ bei thermischer Initiierung wird durch das Geschwindigkeitsgesetz für eine Kinetik erster Ordnung beschrieben:

$$
R_{\mathrm{d}}=-\frac{1}{2} \cdot \frac{\mathrm{d} c_{\mathrm{I}_{2}}}{\mathrm{~d} t}=\frac{\mathrm{d} c_{\mathrm{I}}}{\mathrm{d} t}=2 \cdot k_{\mathrm{d}} \cdot f \cdot c_{\mathrm{I}_{2}}
$$


wobei $c_{\mathrm{I}}$ • die Konzentration des Initiatorradikals, $k_{\mathrm{d}}$ der Geschwindigkeitskoeffizient des Initiatorzerfalls, $f$ die Initiatoreffektivität und $c_{\mathrm{I}_{2}}$ die Initiatorkonzentration darstellen. Die Initiatoreffektivität $f$ bezeichnet den Anteil der Initiatorradikale $I^{\bullet}$, die eine Polymerisation starten, in Bezug auf die Gesamtzahl primär entstehender Radikale. Die Geschwindigkeit der Radikalbildungsreaktion ist in den meisten Polymerisationen geringer als die der Anlagerung an das erste Monomermolekül (Bildung eines Monomerradikals $\mathrm{P}_{1}^{\bullet}$ ) und daher geschwindigkeitsbestimmend. Die Gesamtreaktion zur Bildung von $\mathrm{P}_{1}^{\bullet}$ aus $\mathrm{I}_{2}$ kann damit vereinfachend nur durch den Geschwindigkeitskoeffizient $k_{\mathrm{d}}$ und die Initiatoreffektivität $f$ beschrieben werden.

\subsubsection{Kettenwachstum}

Die Anlagerung des bei der Initiierung bebildeten Monomerradikals $\mathrm{P}_{1}^{\bullet}$ an Monomere führt im weiteren Verlauf zur Bildung der makromolekularen Spezies $\mathrm{P}_{\mathrm{n}}^{\bullet}$ mit einem am Kettenende lokalisierten Radikal. Die zeitliche Änderung der Monomerkonzentration $c_{\mathrm{M}}$ und damit die Wachstumsgeschwindigkeit (Polymerisationsgeschwindigkeit) $R_{\mathrm{p}}$ kann bei gegebener Radikalkonzentration $c_{\mathrm{R}}$ durch folgendes Geschwindigkeitsgesetz beschrieben werden:

$$
R_{\mathrm{p}}=-\frac{\mathrm{d} c_{\mathrm{M}}}{\mathrm{d} t}=k_{\mathrm{p}} \cdot c_{\mathrm{M}} \cdot c_{\mathrm{R}}
$$

Hierbei wird angenommen, dass der Geschwindigkeitskoeffizient der Wachstumsreaktion $k_{\mathrm{p}}$ in einer idealen radikalischen Polymerisation unabhängig von der Länge des Makroradikals und vom Monomerumsatz ist.

\subsubsection{Kettenabbruch}

Die polymerisationsaktiven Spezies, die freien Radikale, sind hochreaktive Teilchen, die miteinander annähernd diffussionskontrolliert reagieren. Der dabei ablaufende Kettenabbruch, der zur Bildung des endgültigen Polymers führt, erfolgt entweder durch Kombination (Geschwindigkeitskoeffizient der Terminierungsreaktion durch Kombination $k_{\mathrm{t}, \mathrm{k}}$ ) oder durch Disproportionierung (Geschwindigkeitskoeffizient der Terminierungsreaktion durch Disproportionierung $k_{\mathrm{t}, \mathrm{d}}$ ) zweier Radikale. Das Verhältnis der Disproportionierung zur Kombination ist vor allem von dem eingesetzten Monomer abhängig. 
Der Terminierungsreaktion zweier Makroradikale müssen die Diffusion der Makroradikalketten zueinander (Translationsdiffusion) sowie die Umorientierung der radikalischen Kettenenden (Segmentdiffussion) vorausgehen [12]. Beide Reaktionsmöglichkeiten werden im Geschwindigkeitskoeffizienten der Terminierungsreaktion $k_{\mathrm{t}}$ zusammengefasst. Für die zeitliche Abnahme der Konzentration des Makroradikals $c_{\mathrm{R}}$ durch Kettenabbruch und damit die Terminierungsgeschwindigkeit $R_{\mathrm{t}}$ wird ein Geschwindigkeitsgesetz zweiter Ordnung angenommen.

$$
R_{\mathrm{t}}=-\frac{\mathrm{d} c_{\mathrm{R}}}{\mathrm{d} t}=2 \cdot k_{\mathrm{t}} \cdot c_{\mathrm{R}}^{2}
$$

Die Geschwindigkeitskoeffizienten der Terminierungsreaktion $k_{\mathrm{t}}$ ist bei üblichen Polymerisationstemperaturen um ca. fünf Zehnerpotenzen größer ist als die Geschwindigkeitskoeffizienten der Propagationsreaktion $k_{\mathrm{p}}$. Die Folge ist im Idealfall eine Schulz-Flory-Verteilung der Kettenlängen mit einem Polydispersitätsindex PDI zwischen 1.5 und 2.0. Der Wert 1.5 für den $P D I$ stellt damit die untere Grenze für radikalische Polymerisationen dar.

\subsubsection{Kettenübertragung}

Eine weitere Reaktion während der Polymerisation ist die Übertragung des aktiven Zentrums auf eine andere Polymerkette, ein Monomer, ein Initiator, ein Lösungsmittelmolekül oder einen gezielt eingesetzten Kettenüberträger bzw. Regler. Dabei wird die radikalische Funktion eines Makroradikals auf ein anderes Molekül X unter Austausch z. B. eines Wasserstoff- oder Halogenatoms übertragen. Für die zeitliche Änderung der Kettenüberträgerkonzentration $c_{\mathrm{X}}$ lässt sich die Geschwindigkeit der Übertragungsreaktion $R_{\mathrm{tr}}$ mit dem folgenden Geschwindigkeitsgesetz beschreiben:

$$
R_{\mathrm{tr}}=-\frac{\mathrm{d} c_{\mathrm{X}}}{\mathrm{d} t}=2 \cdot k_{\mathrm{tr}} \cdot c_{\mathrm{R}} \cdot c_{\mathrm{X}}
$$

Die Übertragungskonstante $C_{\text {tr }}$ kennzeichnet das Verhältnis von Geschwindigkeitskoeffizient der Übertragungsreaktion $k_{\text {tr }}$ zum Geschwindigkeitskoeffizient der Wachstumsreaktion $k_{\mathrm{p}}$ :

$$
C_{\mathrm{tr}}=\frac{k_{\mathrm{tr}}}{k_{\mathrm{p}}}
$$


Die Übertragungsreaktion ändert nicht die Radikalkonzentration. Somit ändert sich die Bruttopolymerisationsgeschwindigkeit auch nicht. Die kinetische Kette, die als die Zahl der Additionsschritte des Monomers an das Radikal definiert ist, bleibt erhalten, während die Polymerkette durch den Kettenüberträger abgebrochen und der Polymerisationsgrad dadurch begrenzt wird.

\subsubsection{Gesamtreaktion}

Bei einer chemisch initiierten Polymerisation wird die Radikalkonzentration als quasistationär angenommen (Bodensteinsches Quasistationaritätsprinzip). Damit die Radikalkonzentration unverändert bleibt, müssen die Radikalbildungsgeschwindigkeit $R_{\mathrm{d}}$ und die Kettenabbruchsgeschwindigkeit $R_{\mathrm{t}}$ gleich sein.

$$
2 \cdot k_{\mathrm{d}} \cdot f \cdot c_{\mathrm{I}_{2}}=2 \cdot k_{\mathrm{t}} \cdot c_{\mathrm{R}}^{2}
$$

Setzt man nun den daraus berechneten Ausdruck für die Radikalkonzentration in das Geschwindigkeitsgesetz des Kettenwachstums ein, erhält man die folgende Gleichung für die Polymerisationsgeschwindigkeit $R_{\mathrm{p}}$

$$
R_{\mathrm{p}}=-\frac{\mathrm{d} c_{\mathrm{M}}}{\mathrm{d} t}=k_{\mathrm{p}} \cdot\left(f \cdot \frac{k_{\mathrm{d}}}{k_{\mathrm{t}}}\right)^{0.5} \cdot c_{\mathrm{M}} \cdot c_{\mathrm{I}}^{0.5}
$$

Daraus folgt, dass die Bruttopolymerisationsgeschwindigkeit außer von den Geschwindigkeitskoeffizienten von der Wurzel der eingesetzten Initiatorkonzentration und von der Monomerkonzentration in erster Ordnung abhängt. Man bezeichnet dieses Gesetz auch als „Wurzelgesetz der radikalischen Polymerisation“.

\subsubsection{Polymerisationsgrad}

Der Polymerisationsgrad wird durch das Verhältnis von Wachstumsgeschwindigkeit $R_{\mathrm{p}}$ zu Abbruchsgeschwindigkeit $R_{\mathrm{t}}$ bestimmt:

$$
\bar{P}_{\mathrm{n}}=\frac{R_{\mathrm{p}}}{R_{\mathrm{t}}}=\frac{k_{\mathrm{p}} \cdot c_{\mathrm{M}} \cdot c_{\mathrm{R}}}{2 \cdot k_{\mathrm{t}} \cdot c_{\mathrm{R}}^{2}}
$$

wobei $\bar{P}_{\mathrm{n}}$ den Zahlenmittel des Polymerisationsgrads bezeichnet. 
Das Konzept der „lebenden“ radikalischen Polymerisation besteht zu einem wesentlicher Teil darin, durch eine verminderte Radikalkonzentration die Abbruchreaktion zurückzudrängen. Mit abnehmender Radikalkonzentration nimmt die Wahrscheinlichkeit der Abbruchsreaktion im Quadrat und die der Wachstumsreaktion nur linear ab, so dass $\bar{P}_{\mathrm{n}}$ gegen unendlich strebt. Die Abbruchreaktionen finden weiter mit einer endlichen Wahrscheinlichkeit statt, werden aber so stark unterdrückt, dass die Zahl der abgebrochenen Polymerketten vernachlässigbar klein ist. Wegen des „,toten“ Polymers spricht man nicht von einer lebenden, sondern von einer kontrollierten radikalischen Polymerisation, die im folgenden Abschnitt detailliert diskutiert wird.

\subsection{Kontrollierte radikalische Polymerisation}

In den letzten zwei Jahrzehnten ist die Übertragbarkeit der Eigenschaften lebender ionischer Polymerisationen auf die freie radikalische Polymerisation intensiv untersucht worden. Durch die Entwicklung neuer Verfahren hat das Interesse an dieser Fragestellung weiter zugenommen. Man erhofft sich dabei, die Vorteile der lebenden Polymerisation, die zu Polymeren mit geringer Polydispersität und sehr vielseitigen Architekturen führt, mit den Vorteilen der radikalischen Polymerisation zu vereinen, die sich durch eine Vielzahl von polymerisierbaren Monomeren sowie durch Unempfindlichkeit gegenüber Verunreinigungen und Wasser auszeichnet.

\subsubsection{Allgemeines Prinzip}

Da die Radikale sehr reaktiv sind, kann der Abbruch von aktiven Ketten durch Terminierungsreaktion dadurch verhindert werden, dass die Konzentration der aktiven Spezies sehr klein gehalten wird (typisch: $c=10^{-9} \mathrm{~mol} \cdot \mathrm{L}^{-1}$, vgl. mit konventioneller radikalischer Polymerisation: $\left.c=10^{-6} \mathrm{~mol} \cdot \mathrm{L}^{-1}\right)$. Zu diesem Zweck wird der Reaktion ein Agens zugesetzt, das sogenannte „schlafende“ Radikale mit den wachsenden Polymerketten bildet. Die Radikale reagieren dabei reversibel zu einer nichtradikalischen Spezies. Der polymerisationsaktive Zustand wird durch Dissoziation dieser schlafenden Radikale wieder hergestellt.

Abbildung 2.4 zeigt das allgemeine Prinzip der kontrollierten radikalischen Po- 


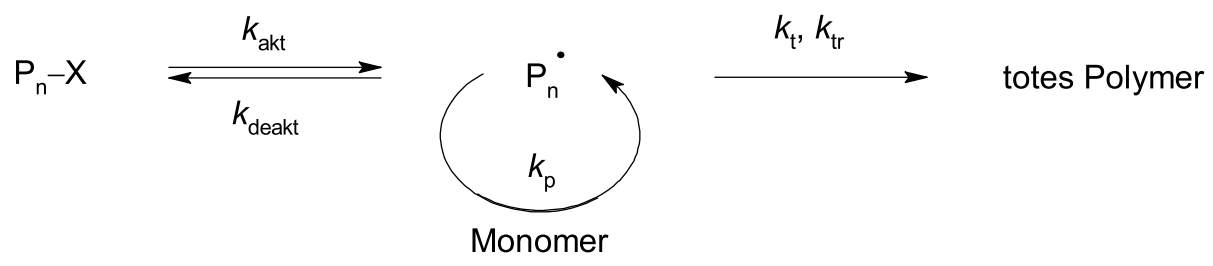

Abbildung 2.4: Schema des zentralen Gleichgewichts bei der kontrollierten radikalischen Polymerisation.

lymerisation, die auf einem dynamischen Gleichgewicht zwischen einer aktiven und inaktiven („schlafenden“) Spezies basiert. Somit liegt nur ein kleiner Teil der reaktiven Spezies als freie Radikale vor, die in einem Wachstumsschritt Monomer anlagern können und dann wieder in das Gleichgewicht eintreten. Dadurch werden Kettenabbruchsreaktionen, die das Kettenwachstum irreversible beenden, minimiert. Gleichzeitig führt das Gleichgewicht zu einem verlangsamten Wachstum der individuellen Polymerketten. Die Lebensdauer der reaktiven Spezies verlängert sich somit deutlich.

Die Eigenschaften der kontrollierten radikalischen Polymerisation hängen mit den Auswirkungen dieses Gleichgewichts auf die gesamte Kinetik der Polymerisation zusammen. Durch das überlagerte Gleichgewicht wird das Kettenwachstum signifikant gegenüber der Initiierung verlangsamt. Dies hat zur Folge, dass alle Radikale zur gleichen Zeit mit dem Wachstum beginnen, was wiederum zu einem simultanen Wachstum aller Polymerketten und somit zu einer einheitlichen Kettenlänge führt. Des Weiteren trägt die längere Lebensdauer dazu bei, dass sich die entstehenden Polymere mit den darin enthaltenen reaktiven Gruppen nach Belieben manipulieren lassen. Sie sind deshalb z. B. als Makroinitiatoren zur Umsetzung eines zweiten Monomers unter Bildung von Blockcopolymeren verwendbar.

Obwohl die neuen Methoden die meisten Eigenschaften einer lebenden Polymerisation beinhalten, finden Kettenterminierung, Kettentransfer und Nebenreaktionen im begrenzten Umfang statt. Die Entwicklung der kontrollierten radikalischen Polymerisation hat das Hauptdefizit der radikalischen Polymerisation nicht bezwungen: ein komplettes Fernbleiben der Kettenterminierung durch bimolekulare Radikalre- 
kombination und Disproportionierung. Konsequenterweise entspricht sie nicht der strengen Definition der lebenden Polymerisation. Man kann zurecht sagen, dass es keine „wirkliche“ lebende radikalische Polymerisation gibt. Deshalb soll die sogenannte lebende radikalische Polymerisation besser als kontrollierte radikalische Polymerisation bezeichnet werden [13].

Um die kontrollierte radikalische Polymerisation in die Terminologie einbinden zu können, wurde von der IUPAC eine weiter gefasste Definition vereinbart. Nach IUPAC gilt, dass die lebende Polymerisation auch die Teilschritte der langsamen Initiierung, der reversiblen Bildung von aktiven Spezies mit verschiedenen Lebensdauern, der reversiblen Bildung von aktiven („schlafenden“) Spezies und/oder der reversiblen Übertragung enthalten können [14]. Zusätzlich zu den Charakteristika der lebenden Polymerisation ist eine kontrollierte radikalische Polymerisation durch folgende Eigenschaften charakterisiert:

- Schneller Austausch zwischen der schlafenden Spezies und dem propagierenden Radikal.

- Ein kleiner Anteil der Ketten $(\prec 10 \%)$ ist an einer Kettenabbruchsreaktion beteiligt.

- Eine schnelle und quantitative Initiierung.

- Eine lineare Zunahme der Molmasse mit dem Monomerumsatz. Eine zu niedrige Molmasse ist ein Indiz für irreversiblen Kettentransfer, während eine zu hohe Molmasse auf die Ineffektivität des Kontrollagens oder auf Kopplungsreaktionen hinweist (siehe Abbildung 2.5).

- Die Polydispersität soll mit dem Monomerumsatz abnehmen. Sie neigt bei höherem Umsatz, und damit bei höherer Viskosität der Polymerisationslösung, wegen der schlechten Mobilität der Reaktionspartner und wegen der progressiven Terminierung zu steigen.

- Die Endgruppenfunktionalität wird nicht von der langsamen Initiierung oder der langsamen Austauschreaktion beeinflusst. Ihr relativer Anteil an dem polymeren Produkt wird von der Kettenabbruchsreaktion reduziert. 


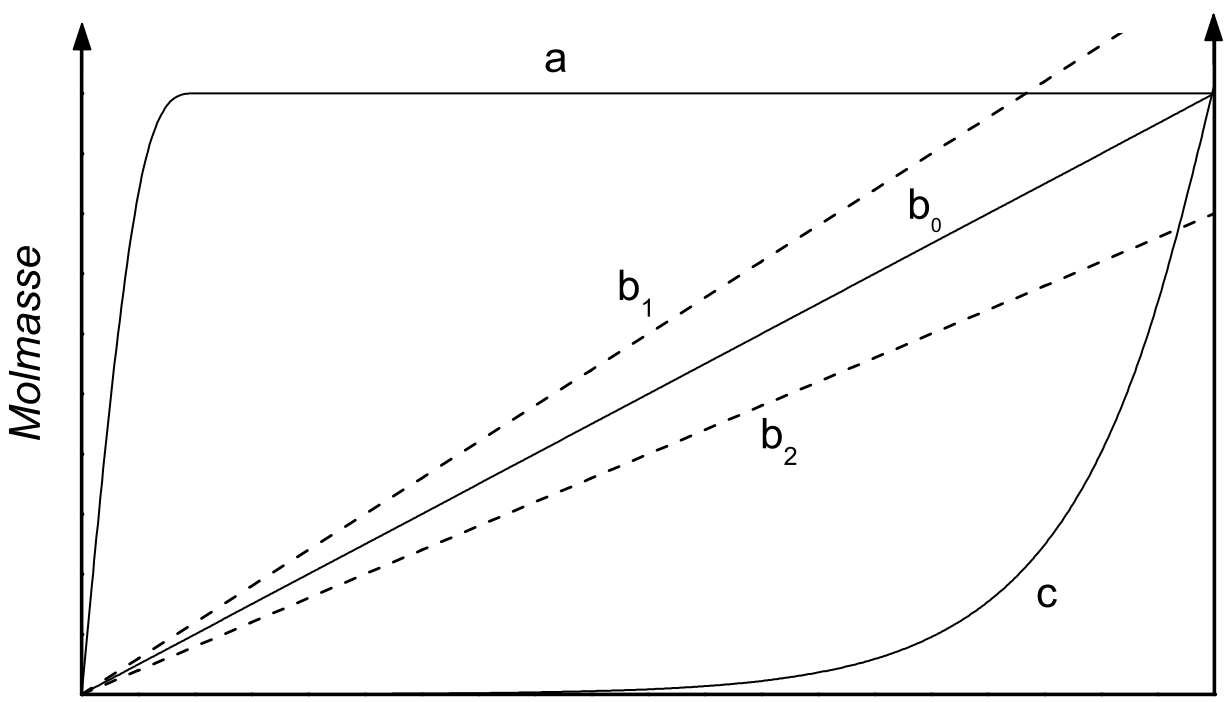

Monomerumsatz

Abbildung 2.5: Schematische Darstellung der Abhängigkeit der Polymermolmasse vom Monomerumsatz bei der konventionellen radikalischen Polymerisation (a), den kontrollierten radikalischen Polymerisationen ( $b_{0}$ ideal, $b_{1}$ bei ineffektivem Kontrollagens und $b_{2}$ bei irreversiblem Kettentransfer) sowie der Polykondensation (c).

Für die Synthese von Polymeren mit enger Molmassenverteilung muss ein Konzept zur kontrollierten radikalischen Polymerisation weitere Kriterien erfüllen. So müssen die Bildung und Spaltung der reversiblen Bindung mit angemessener Geschwindigkeit erfolgen, damit für alle Ketten die gleiche Wachstumswahrscheinlichkeit gegeben ist. Aus dem gleichen Grund sollten zusätzlich alle aktiven Zentren schon zu Beginn der Polymerisation vorhanden sein oder in einer kurzen Initiierungsperiode gebildet werden.

In den letzten 10 Jahren haben das Wissen und das Interesse an kontrollierter radikalischer Polymerisation und ihrer Anwendungen enorm zugenommen, was die sehr starke Zunahme von wissenschaftlichen Veröffentlichungen in diesem Bereich belegt. Diese neue Methode macht es möglich Polymere mit einem PDI $\prec 1.5$ herzustellen, im Gegensatz zur konventionellen Polymerisation wo PDI $\succ 2$ üblich sind. Es existieren mehrere Ansätze für die kontrollierte radikalische Polymerisation, welche 
völlig unterschiedliche Agenzien X zur reversiblen Terminierung der aktiven Zentren nutzen. Zurzeit sind die drei folgenden kontrollierten radikalischen Polymerisationsarten besonders erfolgreich:

1. Kombination und Homolyse: NMP (Nitroxide Mediated Polymerization [15, $16,17])$

2. Übergangsmetall-katalysierter Atomtransfer: ATRP (Atom Transfer Radical Polymerization [18, 4]),

3. Degenerative Kettenübertragung: RAFT-Polymerisation (Reversible Addition Fragmentation Chain Transfer [19, 20]).

Abbildung 2.6 zeigt die berühmtesten molekularen Stellvertreter dieser drei kontrollierten radikalischen Polymerisationssysteme. Im Folgenden werden die drei Polymerisationsmethoden NMP, ATRP und RAFT kurz vorgestellt.

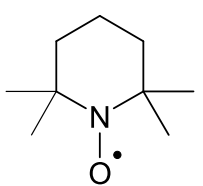

TEMPO<smiles></smiles>

Cumyldithiobenzoat

Abbildung 2.6: Stellvertretende molekulare Spezies, die die radikalische Polymerisation via NMP, ATRP und RAFT-Technik kontrollieren: TEMPO für NMP, CuBr/bipyridinKomplex für ATRP und Cumyldithiobenzoat für RAFT-Polymerisation.

\subsubsection{Kombination und Homolyse : NMP}

Die kontrollierte radikalische Polymerisationsmethode NMP beruht auf dem Prinzip eines reversiblen Kettenabbruchs propagierender Polymerketten durch Kombination mit stabilen Radikalen. Solomon et al. [15] sind die ersten, die ein stabiles freies 


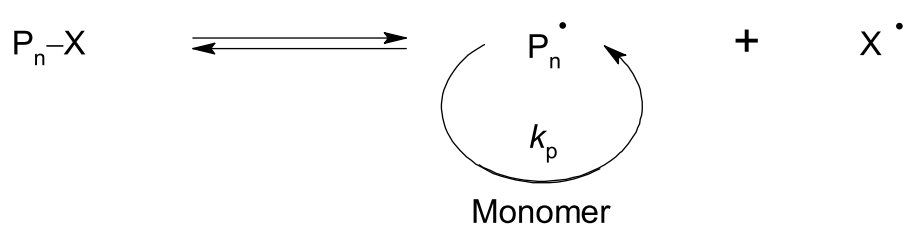

Abbildung 2.7: Reversible Aktivierung durch Homolyse und Kombination. Es entsteht ein Gleichgewicht zwischen den schlafenden Spezies $P_{n}-X$ und einem kohlenstoffzentrierten Radikal $\mathrm{P}_{\mathrm{n}}^{\bullet}$ als aktive Spezies bei der NMP-Polymerisation. $\mathrm{X}^{\bullet}$ ist ein Nitroxidradikal, wie z. B. TEMPO.

Radikal, ein Nitroxidradikal, als reversibles Terminierungsagens verwendet. Die kontrollierte radikalische Polymerisation mit Nitroxiden basiert auf dem Gleichgewicht zwischen der schlafenden Spezies $\mathrm{P}_{\mathrm{n}}-\mathrm{X}$ auf der linken Seite und einem kohlenstoffzentrierten Radikal $\mathrm{P}_{\mathrm{n}}^{\bullet}$ mitsamt dem stabilen Nitroxidradikal $\mathrm{X}^{\bullet}$ auf der rechten Seite (siehe Abbildung 2.7). Die reversible Spaltung der Nitroxyl-Kohlenstoffbindung in der Polymerkette erfolgt thermisch bei Temperaturen über $100{ }^{\circ} \mathrm{C}$. Während das kohlenstoffzentrierte Radikal ein Monomer addieren kann, ist das Nitroxidradikal so stabil, dass es weder ein Monomer addieren, noch mit sich selbst terminieren oder mit einem Makroradikal disproportionieren kann. Es kann ausschließlich mit einem kohlenstoffzentrierten Radikal durch Kopplung reagieren.

Das in Abbildung 2.7 dargestellte Gleichgewicht liegt weit auf der Seite der schlafenden Spezies, des Alkoxyamins $\mathrm{P}_{\mathrm{n}}-\mathrm{X}$. Dadurch ist die Konzentration an freien Radikalen $\mathrm{P}_{\mathrm{n}}^{\bullet}$ sehr gering. Bevor ein freies Radikal wieder durch ein Nitroxidradikal deaktiviert wird, kann es einige Wachstumsschritte durchlaufen, wobei die Zahl der Wachstumsschritte pro Radikal im Durchschnitt gleich ist. Dadurch kommt es zur Bildung eines nahezu monodispersen Polymers.

Die Verwendung von funktionellen Alkoxyaminen als unimolekulare Initiatoren ist sehr effizient im Hinblick auf die Kontrolle der Molmasse und der Endgruppenfunktionalität eines Vinylpolymers. Schon durch die Verwendung des kommerziell erhältlichen 2,2,6,6-Tetramethylpiperidinoxyl (TEMPO) lassen sich erfolgreich Polymere unterschiedlicher Strukturen synthetisieren. Die meisten Veröffentlichungen berichten über die Synthese von Polystyrol oder Copolymere auf Basis von Sty- 
rol, die durch NMP-Technik enge und kontrollierte Molmassenverteilung vorweisen. Aber nur wenige Veröffentlichungen weisen auf die Synthese anderer Polymere hin.

\subsection{3 Übergangsmetall-katalysierter Atomtransfer : ATRP}

Der Mechanismus von ATRP ist in Abbildung 2.8 schematisch dargestellt. Hier wird die schlafende Spezies $\mathrm{P}_{\mathrm{n}}-\mathrm{X}$ durch einen katalytisch aktiven Übergangsmetallkomplex $\mathrm{M}_{\mathrm{t}}^{\mathrm{n}} /$ Ligand aktiviert. Es entsteht ein Gleichgewicht zwischen einer schlafenden und einer aktiven radikalischen Spezies, das auf der reversiblen Redoxreaktion zwischen einem komplexierten Übergangsmetall in niedriger Oxidationsstufe und in der Regel einem Alkylhalogenid basiert. Das Alkylhalogenid wird zu Beginn der Polymerisation homolytisch in ein kohlenstoffzentriertes Radikal $\mathrm{P}_{\mathrm{n}}^{\bullet}$, welches die Polymerisation startet, und in ein Halogenradikal gespalten. Das gebildete Halogenradikal reagiert in einer Redoxreaktion mit dem Übergangsmetall, welches dabei in eine höhere Oxidationsstufe überführt wird $\left(\mathrm{M}_{\mathrm{t}}^{\mathrm{n}+1}\right)$. Das dabei gebildete Halogenid-Ion wird anschließend im Übergangsmetallkomplex gebunden. Während der Rückreaktion reagieren die aktiven Makroradikale mit dem Übergangsmetallkomplex in der Weise, dass ein polymeres Alkylhalogenid gebildet wird und das Übergangsmetall wieder in der niedrigeren Oxidationsstufe vorliegt. Die Wiederholung dieser Prozesse ermöglicht die Bildung eines monodispersen Polymers.

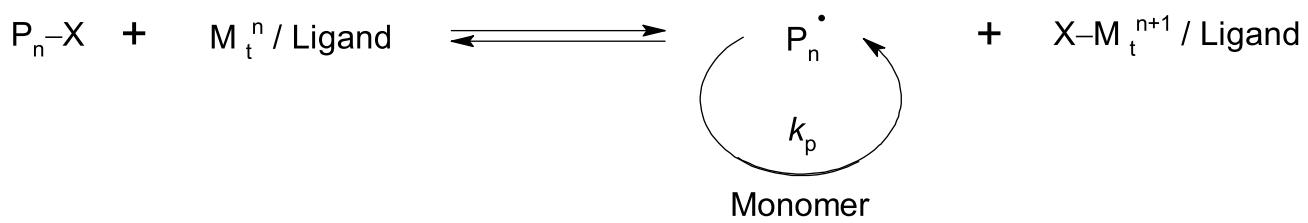

Abbildung 2.8: Schematische Darstellung des Gleichgewichts bei der ATRP-Polymerisation

Sowohl NMP als auch ATRP unterliegen dem von Fischer erstmals berichteten „Persistent-Radical-Effect“"[21]:

Zu Beginn der Reaktion ist die Konzentration des Radikals und des Übergangsmetalls in höherer Oxidationsstufe $\mathrm{M}_{\mathrm{t}}^{\mathrm{n}+1}$ gleich Null. Durch das Anspringen der 
Reaktion in der Vorgleichgewichtsphase nehmen die Konzentration der beiden Spezies zu, wobei es sich bei den Radikalen um eine vergängliche kurzlebige Spezies handelt, während das Übergangsmetall $\mathrm{M}_{\mathrm{t}}^{\mathrm{n}+1}$ nur mit der aktiven Spezies reagiert und als beständig bzw. persistent bezeichnet wird.

Während dieser ersten Reaktionsschritte ist die Konzentration an Radikalen sehr hoch, und die Geschwindigkeit, mit der die Radikale in die schlafenden Spezies überführt werden, ist geringer als die Geschwindigkeit der Terminierung durch Kombination- oder Disproportionierungsreaktionen zweier Radikale.

Mit jeder Terminierungsreaktion nimmt die Radikalkonzentration ab und die des Übergangsmetalls $\mathrm{M}_{\mathrm{t}}^{\mathrm{n}+1} \mathrm{zu}$. Ist eine genügend hohe Konzentration an $\mathrm{M}_{\mathrm{t}}^{\mathrm{n}+1}$-Spezies erreicht, so wird die Reaktion zur schlafenden Spezies schneller als die Abbruchsreaktionen und ein kontrollierter Verlauf der Polymerisation wird möglich.

Eine endliche Konzentration an Radikalen während des Kettenwachstums bedeutet, dass Abbruchsreaktionen zwar minimiert, jedoch nie vollständig unterdrückt werden können. Da die $\mathrm{M}_{\mathrm{t}}^{\mathrm{n}+1}$-Spezies persistent ist und ihre Konzentration im Laufe der Polymerisation zunimmt, sollte sich das Gleichgewicht immer stärker auf die Seite der schlafenden Spezies verschieben und die Polymerisation dadurch verlangsamen. Berechnungen von Fischer sagen deshalb eine nichtlineare Kinetik erster Ordnung im Bezug auf den Monomerumsatz voraus [22].

\subsubsection{Degenerative Kettenübertragung : RAFT}

Die RAFT-Polymerisation ist die neueste Entwicklung im Forschungsfeld der kontrollierten radikalischen Polymerisation und ist wohl die vielfältigste kontrollierte Polymerisationsmethode hinsichtlich Monomere und Reaktionsbedingungen. Sowohl die kontrollierte radikalische Polymerisationsmethode RAFT als auch NMP vermeiden den sonst unumgänglichen Einsatz von Übergangsmetall-Katalysatoren bei der ATRP-Technik, die sich gerade bei polaren und geladenen Polymeren nur schwierig abtrennen lassen und damit toxikologische Probleme aufwerfen können.

Abbildung 2.9 zeigt vereinfacht das Hauptgleichgewicht der RAFT-Polymerisation das nach dem Degenerativen-Kettenübertragungs-Mechanismus verläuft. Der Mechanismus erfolgt hier allerdings nicht durch die bloße Bindung und Abspaltung der Endgruppe, sondern durch degenerative Transferreaktionen, in denen die 


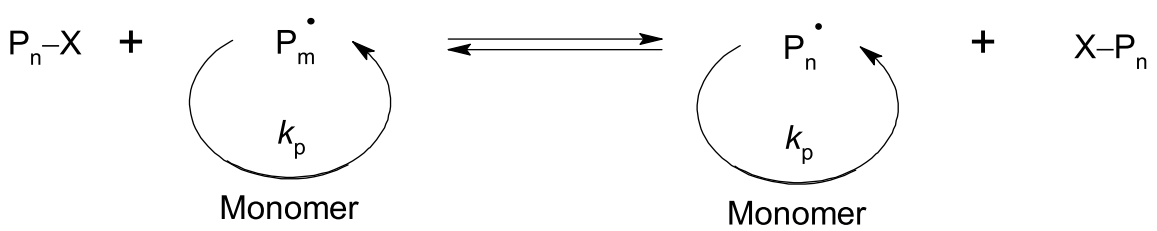

Abbildung 2.9: Schematische Darstellung des bestimmenden Gleichgewichts beim Degenerativen-Kettenübertragungs-Mechanismus der RAFT-Polymerisation.

Endgruppe ständig auf verschiedene aktive Spezies übertragen wird. In diesem Fall wird die schlafende Spezies $\mathrm{P}_{\mathrm{n}}-\mathrm{X}$ von einem wachsenden Radikal $\mathrm{P}_{\mathrm{m}}^{\bullet}$ angegriffen, wobei wieder ein wachsendes Radikal $\mathrm{P}_{\mathrm{n}}^{\bullet}$ und eine schlafende Spezies $\mathrm{P}_{\mathrm{m}}-\mathrm{X}$ entsteht. Der Nettoeffekt ist eine Übertragung der Gruppe X von einem Radikal auf ein anderes. Einige kontrollierte radikalische Polymerisationsprozesse, z. B. mittels Dithioester, Alkyliodid oder Alkyltellurid, basieren auf diesen DegenerativenKettenübertragungs-Mechanismus. Die Kinetik der RAFT-Polymerisation ist sehr komplex und lässt sich nur durch eine Vielzahl von Faktoren erklären. Sie wird im Abschnitt 2.5 detailliert beschrieben.

\subsubsection{Vergleich der kontrollierten Polymerisationsmethoden}

Die drei vorgestellten Methoden der kontrollierten radikalische Polymerisation sind aufgrund intensiver Forschung in ihrer Entwicklung weit fortgeschritten, und das Verständnis der Mechanismen ist, auch wenn im Detail noch nicht vollständig, doch sehr gut.

Die NMP mit kommerziell erhältlichem TEMPO weist verglichen mit anderen kontrollierten Methoden eine geringe Polymerisationsgeschwindigkeit auf. Auch die Monomerauswahl ist noch sehr eingeschränkt. Durch Einsatz neuer Nitroxide, die teuer und sehr aufwendig in der Herstellung sind, ist die Monomervielfalt verbessert worden.

Die ATRP lässt sich auf eine große Anzahl von Vinylmonomeren anwenden, um Polymer mit enger Molmassenverteilung, komplexer Topologie und Zusammensetzung herzustellen. Die große Bandbreite an Initiator/Katalysatorsystemen macht sie 
flexibel in der Wahl der Reaktionsbedingungen wie Temperatur und Lösemittel. Das Katalysatorsystem ist aber zugleich das schwerwiegendste Problem dieser Technik. Zum einen ist eine einfache Verwendung der zurzeit für die radikalische Polymerisation verwendeten Stahlapparaturen in der Industrie nicht möglich, weil es zu unerwünschten Redox-Prozessen zwischen Metallsalz und Eisen kommt. Zum anderen benötigt der ATRP-Prozess 0.1 bis 1 Gew.\% an Übergangsmetall, das im Polymerprodukt verbleibt, dessen Reinigung schwierig ist. Die Kontaminierung des Polymers mit Metall begrenzt wahrscheinlich die industrielle Anwendung dieser Technik. Um dieses Problem zu beseitigen wurden verschiedene Lösungsansätze entwickelt, wie z. B. Immobilisierung des Katalysators auf Silica [23] oder vernetztem Polystyrol für die Katalysatorabtrennung und das Katalysatorrecycling [24]. Neuere, viel versprechende Entwicklungen zur Reduzierung der Katalysatormenge sind AGET-ATRP (Activator Generated by Electron Transfer [25]) und ARGET-ATRP (Activator ReGenerated by Electron Transfer [26]).

Der RAFT-Prozess lässt sich ebenfalls auf eine breite Vielfalt von Monomeren unter verschiedensten Reaktionsbedingungen anwenden. Die kontrollierte Polymerisation von besonders reaktiven Monomeren wie Vinylacetat ist nur mit der RAFTTechnik möglich. Jedoch müssen die verwendeten RAFT-Agenzien auf die Monomere angepasst werden. Diese Transferreagenzien sind nicht käuflich zu erwerben und müssen im Labor mehr oder weniger teuer und aufwendig synthetisiert werden. Ein weiterer Nachteil ist die intensive Färbung der RAFT-Agenzien, die zu gefärbten Produkten führen. Außerdem kann der Einsatz von schwefelhaltigen Verbindungen zudem zu einer unerwünschten Geruchsbelästigung führen. Bei der Synthese von bioorganischen-synthetischen Konjugaten hat die RAFT-Polymerisation gegenüber der ATRP klare Vorteile, denn es ist davon auszugehen, dass zwischen Dithioestern und Peptiden eine geringere Wechselwirkung besteht als zwischen Übergangsmetallen basierenden ATRP-Katalysatoren und Peptiden.

Da die RAFT-Polymerisation den Schwerpunkt in dieser Arbeit stellt, werden ihre Grundlagen im nächsten eigenen Abschnitt ausführlich beschrieben und damit hervorgehoben. Es soll noch angemerkt werden, dass die NMP und ATRP deswegen keineswegs schlechter oder nachteilig beim Vergleich mit RAFT-Polymerisation ist. Für manche Anwendungen sind NMP- und ATRP-Technik wegen ihre längeren 
Entwicklungszeit und der Patentschutzsituation genauso gut oder besser geeignet als die junge RAFT-Technik.

\subsection{Grundlagen der RAFT-Polymerisation}

Eine viel versprechende Methode, eine kontrollierte radikalische Polymerisation von Vinylmonomeren auszuführen ist der „Reversible Addition Fragmentation Chain Transfer (RAFT)“-Prozess. Diese relativ junge Technik wurde in Australien von einem Team der Commonwealth Science \& Industrial Research Organization (CSIRO), angeführt von Rizzardo, im Jahr 1999 zum ersten Mal veröffentlicht [27, 28].

\subsubsection{Kinetik der RAFT-Polymerisation}

In Abbildung 2.10 ist das kinetische Schema einer RAFT-Polymerisation zu sehen. Die Elementarschritte einer konventionellen radikalischen Polymerisation sind lediglich von zwei RAFT-Gleichgewichten überlagert. Im reversiblen Vorgleichgewicht (II.) kommt es über die Addition eines Primärradikals I` oder eines wachsenden Radikals $\mathrm{P}_{\mathrm{n}}^{\bullet}$ an das RAFT-Reagens $\mathbf{1}$ zur Bildung eines intermediären RAFT-Radikals $\mathbf{2}$, welches entweder zurück zu den Edukten oder unter Freisetzung der Abgangsgruppe $\mathrm{R}^{\bullet}$ fragmentiert. Wichtig für den Mechanismus ist, dass das RAFT-Radikal durch die Z-Gruppe stabilisiert wird.

Das abgehende Radikal $\mathrm{R}^{\bullet}$ ist seinerseits in der Lage wieder an das polymere RAFT-Agens zu addieren oder durch Reinitiierung ein wachsendes Polymerradikal zu bilden. Wenn das Radikal $\mathrm{R}^{\bullet}$ ein Wachstum initiiert hat, sodass sich bei einer erneuten Addition die potentiellen Abgangsgruppen nicht mehr wesentlich unterscheiden, so befindet sich das System nicht mehr im Vor- sondern im Hauptgleichgewicht.

Im Hauptgleichgewicht addiert ein wachsendes Radikal $\mathrm{P}_{\mathrm{n}}^{\bullet}$ reversibel an ein polymeres RAFT-Reagenz. Über die Ausbildung des intermediären RAFT-Radikals 4 kommt es erneut zur Fragmentierung in ein polymeres RAFT-Reagenz und in ein wachsendes Radikals $\mathrm{P}_{\mathrm{m}}^{\bullet}$. Das wachsende Radikal $\mathrm{P}_{\mathrm{m}}^{\bullet}$ kann seinerseits von neuem an das polymere RAFT-Reagenz addieren. Aufgrund der schnellen Geschwindigkeit der Additions- und Fragmentierungsreaktion sind meist alle wachsenden Ketten an einem RAFT-Gleichgewicht beteiligt und liegen nicht in freier Form vor. Sie sind nur 
I. Initiierung

I.a

$\mathrm{I}_{2} \underset{f}{\stackrel{k_{\mathrm{d}}}{\longrightarrow}} 2 \mathrm{I}^{\circ}$

I.b

I. $\underset{\text { Monomer }}{\stackrel{k_{\text {pini }}}{\longrightarrow}} \mathrm{P}_{1}^{\text {. }}$

II. Vorgleichgewicht<smiles>[R][PH]#[Y10]C([Z])S[R]</smiles>

III. Propagation

III.a

$$
\mathrm{P}_{\mathrm{n}}^{\cdot} \underset{\text { Monomer }}{\stackrel{k_{\mathrm{p}}}{\longrightarrow}} \mathrm{P}_{\mathrm{n}+1}^{\cdot} \quad \text { III.b } \quad \mathrm{R}^{\cdot} \underset{\text { Monomer }}{\stackrel{k_{\mathrm{p} \text {,reini }}}{\longrightarrow}} \mathrm{P}_{1}^{\cdot}
$$

IV. Hauptgleichgewicht<smiles></smiles>

V. Terminierung

V.a

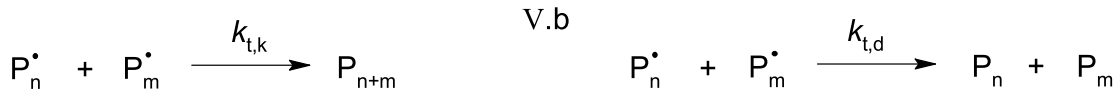

Abbildung 2.10: Kinetisches Schema der RAFT-Polymerisation

nach der Fragmentierung kurzzeitig in der Lage durch Anlagerung an ein Monomermolekül weiter zu wachsen. Da nun in der kontrollierten RAFT-Polymerisation alle Polymere nahezu gleich wachsen, ist eine chemische Unterscheidung der am Hauptgleichgewicht beteiligten Polymerradikale $\mathrm{P}_{\mathrm{m}}^{\bullet}$ und $\mathrm{P}_{\mathrm{n}}^{\bullet}$ nicht notwendig. Für die beiden wachsenden Radikale ist eine gleichgroße Wahrscheinlichkeit gegeben, mit einem Monomer zu reagieren, womit eine enge Molmassenverteilung erreicht wird.

Das Gleichgewicht zwischen aktiver und schlafender Spezies wird durch das Verhältnis von Initiator und RAFT-Reagenz vorgegeben. Es darf nur eine sehr geringe Menge Initiator eingesetzt werden, wenn ein gut kontrollierter Polymerisati- 
onsverlauf erreicht werden soll. Am Ende der Polymerisation besitzen nahezu alle Polymerketten eine dem eingesetzten RAFT-Agens entsprechende aktive Endgruppe, die wieder reaktiviert werden kann, um z. B. Blockcopolymere herzustellen.

Der Polymerisationsgrad $\bar{P}_{\mathrm{n}}$ wird durch das Verhältnis der Konzentration von Monomer $[\mathrm{M}]_{0}$ zu RAFT-Agenzien $[\mathrm{RAFT}]_{0}$ unter Berücksichtigung des fraktionellen Monomerumsatzes $U_{\mathrm{M}}$, aber näherungsweise unter Vernachlässigung der Initiatormenge und der irreversiblen Transferreaktionen, vorbestimmt.

$$
\bar{P}_{\mathrm{n}}=\frac{[\mathrm{M}]_{0}}{[\mathrm{RAFT}]_{0}} \cdot U_{\mathrm{M}}
$$

Bei einer idealen RAFT-Polymerisation wird die Molekulargewichtsverteilung durch eine Poisson-Verteilung dargestellt. Die Polydispersität PDI der Polymere kann wie in folgender Gleichung beschrieben werden.

$$
P D I=\frac{M_{\mathrm{w}}}{M_{\mathrm{n}}}=1+\frac{1}{\bar{P}_{\mathrm{n}}}
$$

Die Polydispersität des Polymers ergibt sich aus dem Verhältnis des Gewichtsmittels des Molekulargewichts $M_{\mathrm{w}}$ zu dem des Zahlenmittels $M_{\mathrm{n}}$. Dieses lässt sich auch unter Einbeziehung des Polymerisationsgrades $\bar{P}_{\mathrm{n}}$ ausdrücken und strebt für sehr hohe Polymerisationsgrade gegen eins.

\subsubsection{Retardierung der Polymerisationsgeschwindigkeit}

Da das RAFT-Gleichgewicht die konventionelle radikalische Polymerisation überlagert, wird die Konzentration des propagierenden Radikals nicht verändert. Somit bleibt die Kinetik der konventionellen Polymerisation zumindest im Idealfall unverändert. Sowohl Kettenwachstum als auch Kettenabbruch finden in der RAFTPolymerisation deshalb mit der gleichen Geschwindigkeit wie in der konventionellen Polymerisation statt.

Abhängig von der Struktur des eingesetzten RAFT-Agens kann es aber zu einer Abnahme der Polymerisationsgeschwindigkeit mit steigender RAFT-Agens-Konzentration kommen. Dieser Effekt wird als Retardierung bezeichnet. Bisher konnte noch nicht eindeutig geklärt werden, was genau für den retardierenden Effekt des RAFTAgens verantwortlich ist. Sicher scheint nur, dass Nebenreaktionen des im RAFTGleichgewicht gebildeten intermediären RAFT-Radikals zu dem retardierenden Ef- 


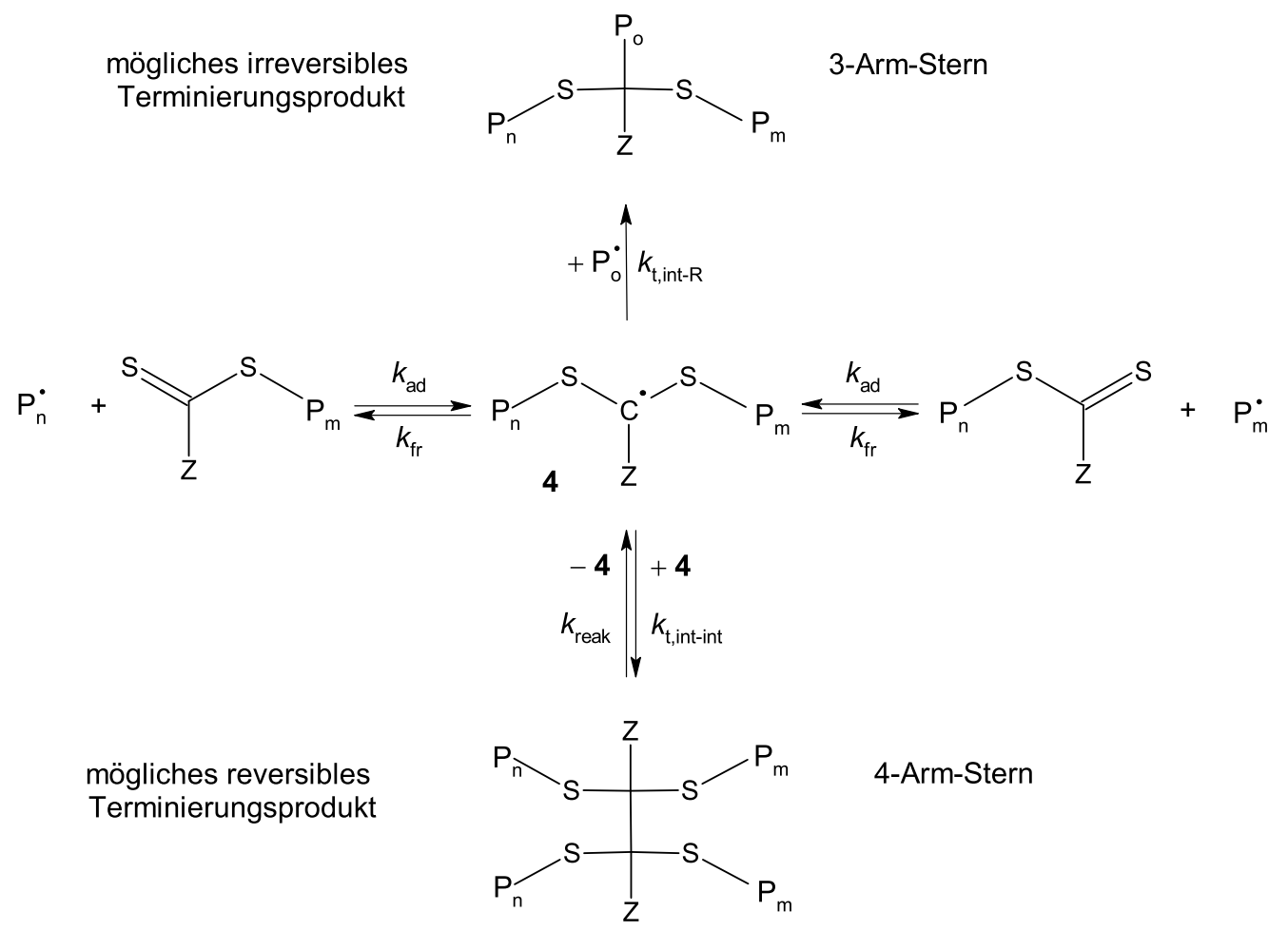

Abbildung 2.11: Schema der Reaktionen, die zum Effekt der Retardierung führen können [29].

fekt beitragen. Der Retardierungseffekt nimmt mit der steigenden Stabilität des intermediären RAFT-Radikals zu.

In Abbildung 2.11 ist ein Schema der zwei unterschiedlichen Mechanismen zu sehen, die möglicherweise zum Effekt der Retardierung führen [29]:

1. Durch Addition eines Polymerradikals an ein polymeres RAFT-Agens wird das intermediäre RAFT-Radikal gebildet. Neben der üblichen Fragmentierung kann das RAFT-Intermediat in einer irreversiblen Terminierungsreaktion mit einem weiteren Polymerradikal oder mit sich selbst reagieren [30]. Durch die irreversible Terminierung gehen Radikale verloren, was die Retardierung verursacht, da die Konzentration des propagierenden Radikals geringer ist. Die von Monteiro et al. [31] stammende Erklärung der irreversiblen Terminierung wurde von Fukuda et al. [32] sowie Buback und Mitarbeiter [33] übernommen. 
2. Andererseits ist denkbar, dass zwei RAFT-Intermediate miteinander ein reversibles Terminierungsprodukt bilden. Diese reversible Terminierungsreaktion erhöht die Lebensdauer des intermediären RAFT-Radikals und verlangsamt damit die Fragmentierungsreaktion. Die Radikale werden als reversibles Terminierungsprodukt gespeichert und stehen für das Kettenwachstum nicht zur Verfügung, was dann die Retardierung verursacht [34, 35]. Die Idee der langsamen Fragmentierung wurde von der Arbeitsgruppe um Davis mit experimentellen Ergebnissen unterstützt [36, 37].

Sowohl die irreversible Terminierung als auch die langsame Fragmentierung können den experimentellen Monomerumsatzverlauf mit der Reaktionszeit gut beschreiben. Jedoch unterscheiden sich die angepassten Fragmentierungsgeschwindigkeitskoeffizienten und somit die Konzentration des intermediären RAFT-Radikals der beiden Mechanismen um mehrere Zehnerpotenzen [38, 29]. Die niedrige Konzentration des intermediären RAFT-Radikals, die experimentell durch ESR-Spektroskopie bestimmt wird, spricht eindeutig für das Modell der irreversiblen Terminierung. Ein Hauptargument gegen eine irreversible Terminierung ist, dass bis heute bei gewöhnlichen RAFT-Polymerisationen keine aus der irreversiblen Terminierung stammenden Produkte, z. B. Drei-Arm-Sterne oder Vier-Arm-Sterne ohne Zweifel nachgewiesen werden können [32, 39, 37].

Die Suche nach polymeren Sternen aus der irreversiblen Terminierung blieb bisher erfolglos, obwohl Untersuchungen zeigen, dass diese Sterne bei typischen Polymerisationstemperaturen stabil sind [41]. Einen Lösungsansatz für die Abwesenheit der Sternpolymere, und somit ein wichtiges Argument für das Modell der irreversiblen Terminierung, liefern Buback und Vana, indem Sie einen weiteren Reaktionszyklus der RAFT-Kinetik hinzufügen [40]. Dabei reagiert das entstehende Drei-ArmSternpolymer mit einem propagierenden Radikal, es entsteht wieder das intermediäre RAFT-Radikal 4 und eine „tote“ inaktive Polymerkette (siehe Abbildung 2.12). Diese Hypothese wurde durch thermodynamische Betrachtungen, Modellierungen und DFT-Rechnungen untermauert [42]. 


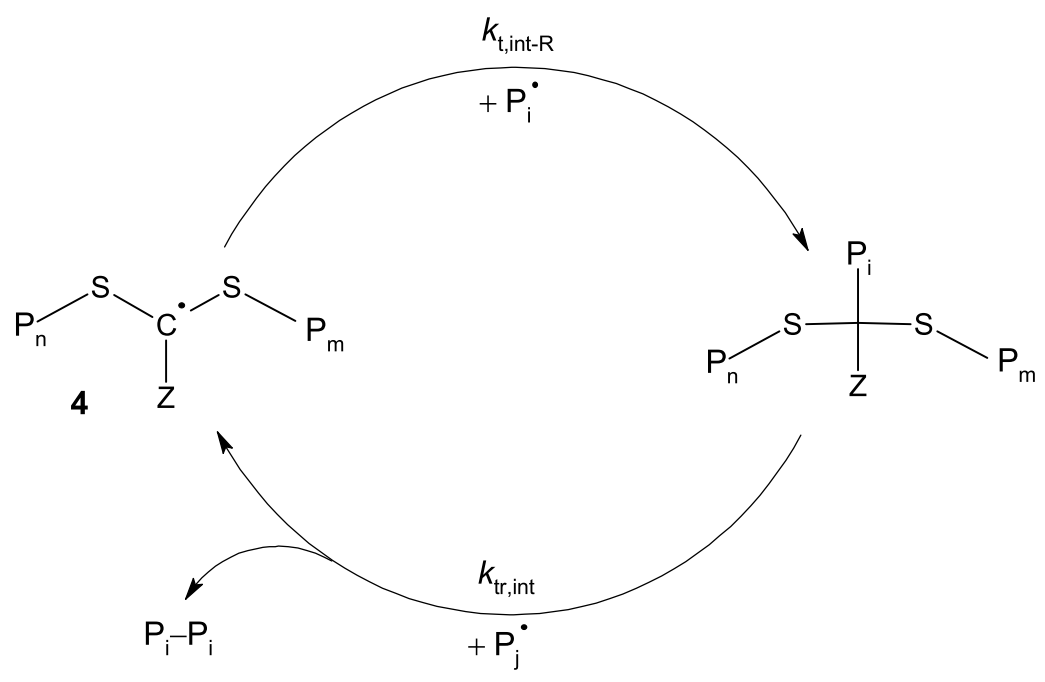

Abbildung 2.12: Schema des Reaktionszyklus, in dem die entstehenden Polymersterne aus der irreversiblen Terminierung vernichtet werden [40].

\subsubsection{Das RAFT-Agens und das Design seiner chemischen Struktur}

Die Technik der RAFT-Polymerisation arbeitet mit schwefelhaltigen Kettenübertragungs-Reagenzien (RAFT-Reagenzien). Durch eine einfache Zugabe eines RAFTAgens zu einem konventionellen radikalischen Polymerisationsprozess wird eine kontrollierte Polymerisation induziert. Derzeit finden folgende Verbindungstypen ihre Anwendung in der RAFT-Polymerisation:

1. Die bekannteste und am häufigsten verwendete Stoffklasse sind die von Rizzardo et al. entwickelten Dithioester, die für eine große Anzahl von Monomeren einsetzbar sind [43]. Wegen der intensiven Färbung des RAFT-Agens ist das gebildete Polymer normalerweise rot. Die rote Färbung zeigt an, dass das aktive Zentrum erhalten bleibt und z. B. für die Synthese von Blockcopolymeren zur Verfügung steht.

2. Die Trithiocarbonate, die zuerst von der CSIRO-Gruppe entwickelt wurden, eignen sich ebenso hervorragend für viele Monomerarten [44]. Ihre meist gelbliche Farbe verursacht eine weniger intensive Färbung des Polymermaterials. 
3. Die Dithiocarbonate (Xanthate) eignen sich besonders für die Polymerisation von Vinylacetaten. Das von Destarac et al. etablierte Verfahren wird auch als MADIX-Prozess bezeichnet (Macromolecular Design via the Interchange of $X$ anthates [45]).

4. Die zyklisch oder aromatisch substituierte N,N-Dithiocarbamate können als RAFT-Agenzien für einen Großteil der gebräuchlichsten Monomere verwendet werden, sie zeigen aber eine etwas geringere Reaktivität [28]. Ihre Transferkonstante ist etwa 100mal kleiner als die der Dithioester.

Der Hauptteil A der vorliegenden Arbeit, die die heterogene RAFT-Polymerisation an der Oberfläche untersucht, wird entsprechend der ersten drei effektivsten Verbindungstypen der RAFT-Agenzien unterteilt. So wird die Verwendung von gebundenen Dithioverbindungen in Kapitel 3, Trithioverbindungen in Kapitel 4 und Xanthaten in Kapitel 5 beschrieben.

In Abbildung 2.13 ist die Grundstruktur der RAFT-Reagenzien zu sehen, wobei mit $\mathrm{R}$ die Abgangsgruppe und mit $\mathrm{Z}$ die stabilisierende Gruppe bezeichnet wird. Für eine effiziente Polymerisation ist die stabilisierende Gruppe Z und die Abgangsgruppe R so auszuwählen, dass die Geschwindigkeitskoeffizienten der am RAFT-Gleichgewicht beteiligten Additionsreaktionen möglichst hoch sind. Je besser die Z-Gruppe das intermediäre Radikal zu stabilisieren vermag, desto höher ist der Geschwindigkeitskoeffizient der Additionsreaktion $k_{\text {ad }}$. Viele RAFT-Reagenzien enthalten einen Phenylring als stabilisierende Gruppe, die das ungepaarte Elektron mittels Mesomerie stabilisiert. Ist der radikalstabilisierende Effekt der Z-Gruppe zu groß, kann die Bildung eines zu stabilen RAFT-Intermediats die Folge sein, was zur Retardierung der Polymerisationsgeschwindigkeit führt [34, 46, 31, 30].

Der Retardierungseffekt, der die Umsatz-Zeit-Ausbeute verringert, wird vermieden, indem die Fragentierungsgeschwindigkeit $k_{\mathrm{fr}}$ erhöht wird. Großen Einfluss auf $k_{\text {fr }}$ in der Anfangsphase der Polymerisation hat die Abgangsgruppe R. Gute Abgangsgruppen bilden stabile Radikale, sind sterisch anspruchsvoll oder haben einen elektrophilen Charakter, was zu einem hohen Wert für $k_{\text {fr }}$ führt.

Abschließend muss darauf geachtet werden, dass das aus der Abgangsgruppe entstehende Radikal $\mathrm{R}^{\bullet}$ genügend reaktiv für eine Re-Initiierung ist. Ist dies nicht der Fall, kann es aufgrund von Terminierungsreaktionen wiederum zur Retardierung der 


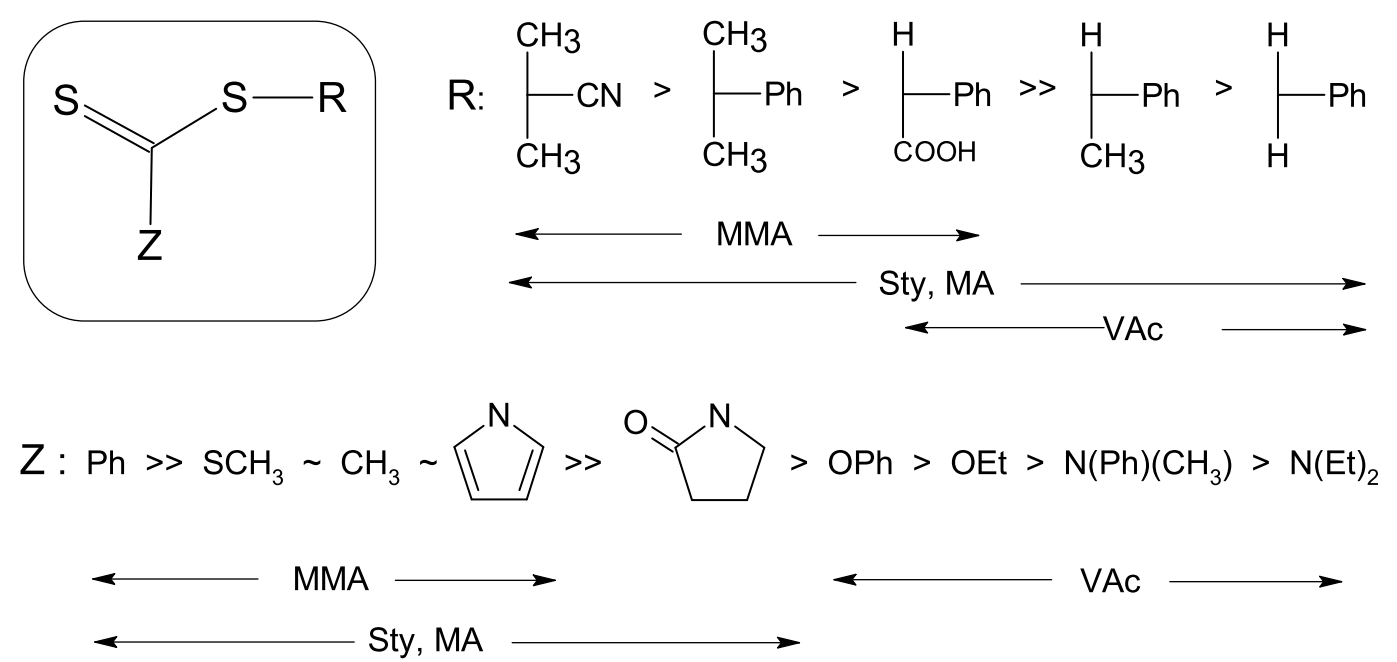

Abbildung 2.13: Allgemeine Leitlinie für das Design von Thiocarbonylthioverbindungen, die als reversible Kettenüberträger in der RAFT-Polymerisation von verschiedenen Monomere fungieren $($ Sty $=$ Styrol, MMA $=$ Methylmethacrylat, MA = Methylacrylat, $\mathrm{VAc}=$ Vinylacetat). Für die Abgangsgruppe R: die Fragmentierungsgeschwindigkeit nimmt von links nach rechts ab. Für die stabilisierende Gruppe Z: die Additionsreaktion nimmt von links nach rechts ab, während die Fragmentierungsgeschwindigkeit zunimmt [20].

Polymerisationsgeschwindigkeit und zu einer langen Induktion in der Anfangsphase der Polymerisation kommen.

Des Weiteren ist anzumerken, dass die Effizienz eines bestimmten RAFT-Agens auch vom Monomer abhängt. Daher ist die effizienteste Kombination von Abgangsgruppe und stabilisierender Gruppe für jedes Monomer neu zu bestimmen, was zu der Vielzahl von verwendeten RAFT-Agenzien führt.

\subsection{Motivation und Zielsetzung}

Wie der Titel „RAFT-Polymerisation an Oberflächen“ bereits erahnen lässt, verfolgt die vorliegende Arbeit das Ziel, den Prozess der RAFT-Polymerisation an der Oberfläche erfolgreich durchzuführen. Dabei wird die Arbeit von zwei Hauptmotivationen vorangetrieben, die mit diesem Prozess gleichzeitig erfüllt werden können (siehe Abbildung 2.14). Zum einen wird ein heterogenes Katalysatorsystem für die 


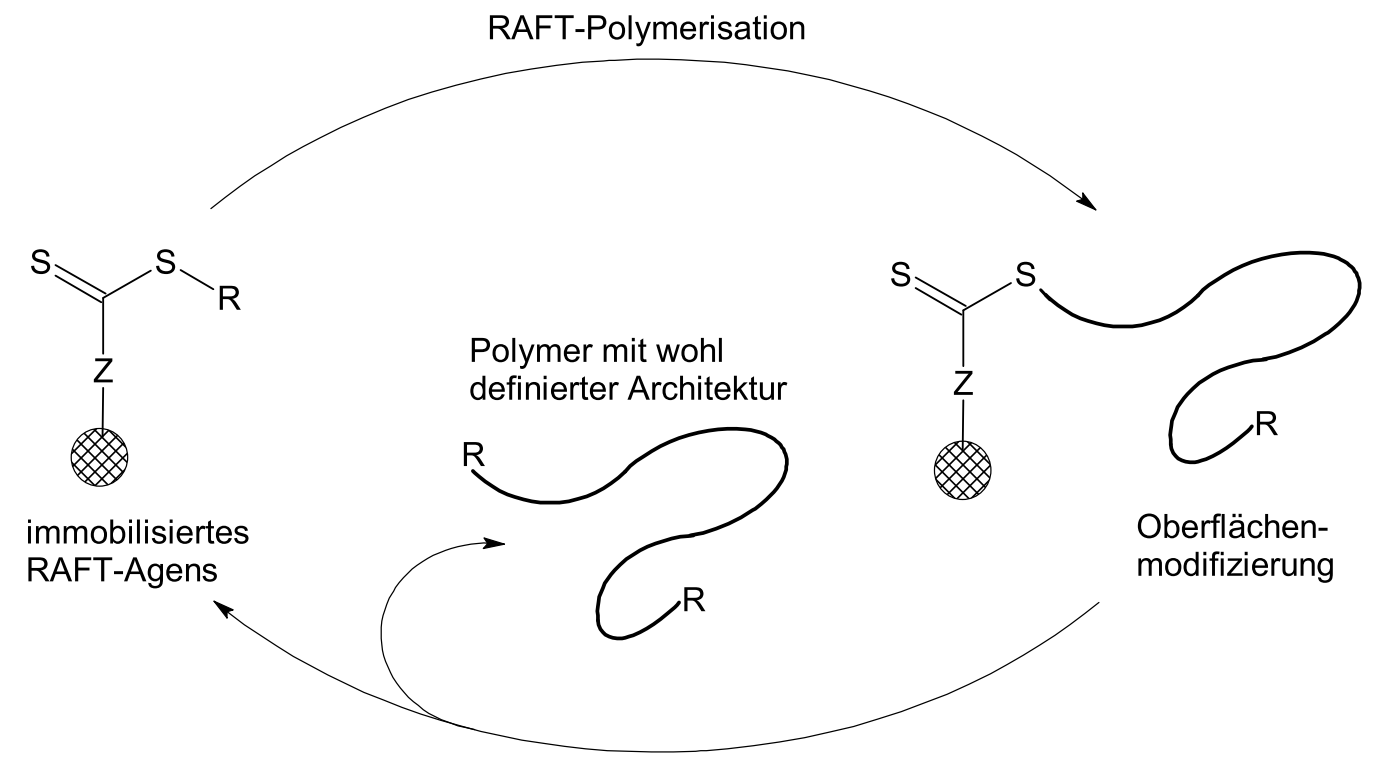

Trennung und Wiedergewinung des RAFT-Agens

Abbildung 2.14: Zielsetzung dieser Arbeit: Heterogenes Katalysesystem für die RAFTPolymerisation und Oberflächemodifizierung mit wohl definiertem Polymer.

RAFT-Polymerisation erforscht und entwickelt und zum anderen wird die Oberfläche eines festen Substrats mit Polymer, das durch die RAFT-Technik eine wohldefinierte Architektur besitzt, modifiziert.

\subsubsection{Heterogene Polymerisations-Katalyse}

Das Wesen einer katalysierten Reaktion besteht darin, dass ein Katalysezyklus auftritt, bei dem Reaktanten umgesetzt und Produkte gebildet werden und der Katalysator schließlich wieder zurückgebildet wird. Das Forschungsfeld der chemischen Katalyse wird in zwei Teilbereiche unterteilt: homogene und heterogene Katalyse. Bei der homogenen Katalyse bilden der Katalysator und die reagierenden Substanzen eine einzige homogene Phase. Ist die katalytische Aktivität auf die Oberfläche eines festen Katalysators beschränkt, so spricht man von einer heterogenen Katalyse.

In der chemischen Verfahrenstechnik werden sowohl homogene als auch heterogene Katalysatoren eingesetzt. Die homogene Katalyse hat jedoch den Nachteil, dass 
es technisch sehr aufwendig ist den Katalysator nach Ablauf der Reaktion von den Produkten wieder abzutrennen. Zudem ist die thermische Stabilität von homogenen Katalysatoren oftmals sehr begrenzt. Der Vorteil von homogenen Katalysatoren liegt jedoch in ihrer höheren Aktivität im Vergleich zu heterogenen Katalysatoren. Die leichte Abtrennbarkeit von den Produkten und weitere verfahrenstechnische Vorteile sind der Grund, warum in industriellen Verfahren heterogene Katalysatoren öfter angewendet werden.

Die Vorteile beider Katalysatortypen lassen sich vereinigen, indem man einen in homogener Phase wirksamen Katalysator an einen unlöslichen Träger bindet. Vorraussetzung ist jedoch, dass der Träger ausreichend thermisch und auch gegenüber den Reaktanten stabil ist und aktive Stellen in ausreichender Anzahl besitzt. Als solche Träger kommen Silica, Aluminiumoxide, Zeolithe und organische hochvernetzte Polymere in Frage.

Systeme von an Festkörpern gebundenen Katalysatoren wurden bereits für kontrollierte Polymerisationen, wie z.B. NMP [47, 48, 49] und ATRP [50, 51, 52] entwickelt. Die meisten Anwendungen des Kontrollagens, das mit einer kovalenten Bindung an der Oberfläche eines festen Substrats gebunden ist, sind auf die Entwicklung von dichten Polymerbürste [53, 54] ausgerichtet, während die Anwendung auf das Konzept der kontrollierten radikalischen Polymerisation mit einem am Substrat fixierten Kontrollagens bisher noch nicht ins Blickfeld des wissenschaftlichen Interesses gerückt ist. Berichte über RAFT-Polymerisation an Oberflächen $[55,56]$ sind spärlich und auch erst vor kurzem veröffentlicht, was sicher in der kurzen Zeitspanne seit Entwicklung der jungen RAFT-Polymerisation begründet liegt.

Um die oben beschriebenen Nachteile der RAFT-Polymerisation zu beheben, ist es unbedingt notwendig, eine neue Methode zu entwickeln, die es ermöglicht das RAFT-Agens nach Ablauf der Polymerisation effektiv vom Polymer abzutrennen und somit wiederzugewinnen. Die Entwicklung eines heterogenen Polymerisationssystems für die RAFT-Polymerisation besitzt ein bisher unerforschtes Potential, die Rückgewinnung des RAFT-Agens zu erleichtern. 


\subsubsection{Oberflächenmodifizierung}

Die Oberflächenbeschaffenheit eines Materials ist für viele seiner Eigenschaften ausschlaggebend. In unserem täglichen Leben spielen dünne Polymerfilme eine immer größere Rolle. DVD, Autolack und die Teflonpfanne sind nur ein paar Beispiele für die zahlreichen und unterschiedlichen Anwendungen. Industriell werden eine ganze Reihe von Verfahren angewendet, um Oberflächeneigenschaften mit Hilfe von Polymeren gezielt zu verändern. Beim sogenannten „spin coating“ wird das Polymer in einem Lösungsmittel gelöst und diese Lösung anschließend auf einen sich drehenden planaren Träger aufgebracht. Außerdem setzt man Verfahren wie Aufsprühen, Lackieren oder das „dip coating“ ein, bei dem das Werkstück durch Eintauchen und Herausziehen in eine Polymerlösung mit einer Schicht überzogen wird. Nachteil dieser Techniken ist, dass die Dicke und Homogenität der Polymerschicht schwierig einzustellen sind. Zudem bilden sich zwischen Substrat und Polymer nur schwache Wechselwirkungen ${ }^{2}$ aus, so dass die Polymerfilme nachträglich vernetzt werden müssen, um ihre Stabilität zu erhöhen [57].

Polymere werden zur Oberflächenmodifizierung in Feldern wie Adhesion, Biomaterialien, Schutzschichten, Bekleidung oder Mikroelektronik eingesetzt. Allgemein gesagt sind spezielle Oberflächeneigenschaften, wie chemische Zusammensetzung, Hydrophilie, Rauheit oder elektrische Leitfähigkeit für eine erfolgreiche Anwendung von Bedeutung. Einfache Polymere wie z.B. Polyethylen besitzen in der Regel diese Oberflächeneigenschaften ursprünglich nicht. Sie haben jedoch exzellente physikalische und chemische Festkörpereigenschaften und sind kostengünstig und relativ einfach herzustellen. Aus diesen Gründen spielen Techniken zur Oberflächenmodifizierung, die es erlauben diese relativ billigen Materialien in wertvolle Endprodukte zu überführen, eine immer wichtigere Rolle in der Industrie.

In der Wissenschaft ist die Beschichtung von Oberflächen mit dünnen und besonders ultradünnen Filmen von molekularer Dicke in den letzten Jahren immer wichtiger geworden. Es wurden zahlreichen Verfahren entwickelt, welche die Modi-

\footnotetext{
${ }^{2}$ Je nach Art der Bindung der adsorbierten Schicht wird zwischen chemischer und physikalischer Adsorption unterschieden. Bei der Chemisorption werden zwischen Adsorbat und dem Festkörper chemische Bindungen ausgebildet, bei Physisorption dagegen wirken hauptsächlich schwache vander-Waals-Kräfte zwischen Adsorbat und Festkörper.
} 
fizierung, Strukturierung und Untersuchung von Oberflächen ermöglichen [58]. Die Forschung und Entwicklung ist darauf fokussiert, den Einsatz von Polymeren in anspruchsvollen technischen Einsatzfeldern möglich zu machen. Dazu müssen Polymere an einer Oberfläche so veredelt werden, dass die Eigenschaften des Verbundsystems dem technischen Anforderungsprofil entsprechen.

Eine einfache Methode zur Herstellung von ultradünnen und stabilen Polymerfilmen besteht darin, das Polymer selbst mit reaktiven Gruppen auszustatten, die wie ein Anker auf der Oberfläche des Trägers anbinden. Eine Alternative hierzu ist, zuerst die Oberfläche des Trägermaterials durch Einsatz niedermolekularer Moleküle zu funktionalisieren. Diese müssen so beschaffen sein, dass sie einerseits eine kovalente Bindung zum Träger eingehen, andererseits aber noch eine reaktive Gruppe besitzen, an welcher das Polymer mittels polymeranaloger Umsetzung gebunden werden kann.

Dünne Polymerfilme können auch mittels Laserdeposition hergestellt werden [59, 60, 61]. Bei der Laserdeposition werden Polymerproben mit einem Laser bestrahlt und das vom Target abgetragene Material auf einem Substrat deponiert. Während der Ablation am Target werden die Polymerketten, abhängig von der verwendeten Energiedichte, oftmals in kleine Bruchteile zerlegt [62, 63].

Neben den bisher beschrieben Methoden, bei denen Polymere auf die Oberfläche eines geeigneten Substrat aufgebracht werden (,grafting to“), gibt es auch die Möglichkeit, Polymere von der Oberfläche wachsen zu lassen („grafting from“). Eine dieser Methoden ist die Plasmapolymerisation, bei der die zu behandelnde Oberfläche in eine durch Unterdruck erzeugte Gasphase eines entsprechenden Monomers gebracht wird. Durch elektrische Entladung werden Radikale im Monomer und auf der Oberfläche erzeugt, die dann die Polymerisation starten. Dieser Prozess verläuft jedoch undefiniert ab. Eine weitere, definierter ablaufende „grafting from“-Methode besteht in der Funktionalisierung der Substratoberfläche mit einem Polymerisationsinitiator durch Selbstorganisationsprozess. Dieser immobilisierte Initiator kann durch thermische oder photochemische Initiierung eine radikalische Polymerisation der entsprechenden Monomere auslösen.

Durch Immobilisierung des Kontrollagens, das die radikalische Polymerisation effektiv kontrolliert, können stabile und wohl definierte Polymerfilme im Nanometer- 
Maßstab erzeugt werden. Verschiedene Techniken zur Herstellung neuartiger Polymerfilme sind mittels NMP, ATRP und RAFT-Polymerisation publiziert. Diese Arbeit soll einen Beitrag zu diesem sich dynamisch entwickelnden Forschungsfeld leisten. Dabei sollen die Fragestellung auf den detaillierten Mechanismus der RAFTPolymerisation an der Oberfläche fokussiert werden, was bisher nur bruchstückhaft beschrieben ist.

\subsubsection{Weitere Motivationen}

Das Ziel dieser Arbeit ist es, zahlreiche Eigenschaften von Polymeren kontrolliert beeinflussen zu können, um polymere Materialien gezielt an bestimmte Anwendungen anpassen zu können. Neben den beiden Hauptmotivationen, Entwicklung eines heterogenen Katalysesystems für die RAFT-Polymerisation und Oberflächenmodifizierung, werden im Rahmen dieser Arbeit noch folgende interessante Aspekte untersucht, die sich aus den Arbeiten an den Hauptzielen hergeben haben:

- Kinetik der RAFT-Polymerisation: Eine erfolgreiche Anwendung der RAFTPolymerisation setzt ein Verständnis der Kinetik dieses Prozesses voraus, was noch nicht gänzlich der Fall ist.

- Herstellung neuartiger Polymermaterialien: Die kontrollierte radikalische Polymerisation eröffnet seit ihrer Entdeckung der Materialwissenschaft neue Wege, eine Vielzahl von vielseitigen und neuartigen Polymermaterialien herzustellen.

In den folgenden Kapiteln werden die unterschiedlichen Strategien und Vorgehensweisen zur Erreichung der beschriebenen Ziele vorgestellt. In Teil A bwz. in Kapitel 3 bis 5 wird die heterogene RAFT-Polymerisation mit verschiedenen gebundenen RAFT-Agenzien beschrieben. In Teil B bzw. in Kapitel 6 und 7 wird die homogene RAFT-Polymerisation dargestellt, die einen Beitrag zur Aufklärung der Kinetik des RAFT-Mechanismus und zur Entwicklung neuartiger Polymermaterialien leisten. 


\section{Teil A}

\section{Heterogene}

\section{RAFT-Polymerisation}





\section{Kapitel 3}

\section{An Silica gebundenes Dithiobenzoat}

In diesem Kapitel wird die heterogene RAFT-Polymerisation von Styrol bzw. von Methylmethacrylat beschrieben. Das Dithiobenzoat, welches die Polymerisation effektiv kontrolliert, ist mit der stabilisierenden Phenylgruppe an die Silica-Oberfläche chemisch festgebunden, um die anschließende Abtrennung des RAFT-Agens von Polymermaterial und seine Wiedergewinnung zu ermöglichen.

\subsection{Einführung}

Die radikalische Polymerisation wurde durch die Entdeckung der kontrollierten lebenden Polymerisationstechnik revolutioniert. Diese Technik erlaubt eine sehr effiziente Kontrolle der Polymerisation im Hinblick auf die Molmasse und die molekulare Architektur. Demzufolge ist die Herstellung von synthetischem Polymer mit niedriger Polydispersität und mit komplexer Mikrostruktur relativ einfach. Unter den vielen kontrollierten Polymerisationstechnik sind NMP ( $N$ itroxide $M$ ediated Polymerization [15, 17]), ATRP (Atom Transfer Radical Polymerization [18, 4]) und RAFT-Polymerisation (Reversible Addition Fragmentation Chain Transfer [19, 20]) die bekanntesten. Sie erlauben die Herstellung von Polymermaterial mit neuartigen Eigenschaften für hochtechnologische Anwendungen.

Die RAFT-Polymerisation, die von Rizzardo et al [27, 28] im Jahr 1999 entdeckt wurde, ist die neueste Entwicklung in diesem Forschungsfeld. Sie ist wohl die 
Vorgleichgewicht:

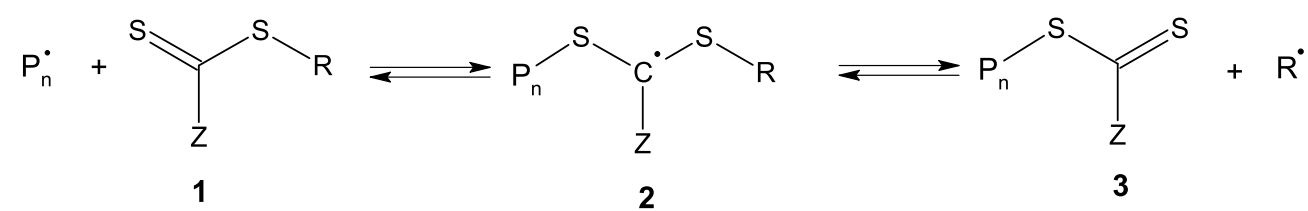

Hauptgleichgewicht:

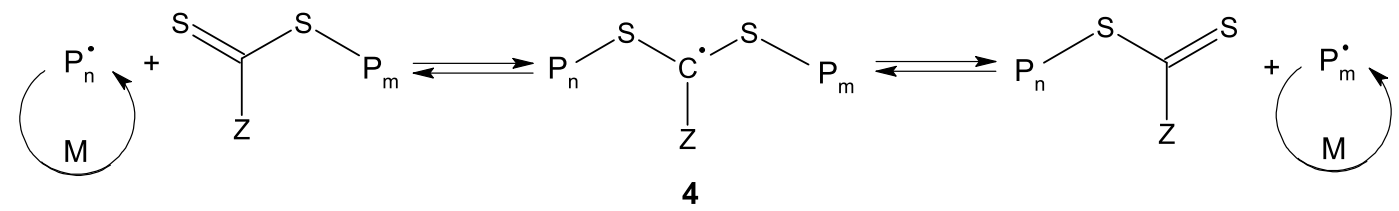

Abbildung 3.1: Vor- und Hauptgleichgewichte in der RAFT-Polymerisation.

vielfältigste kontrollierte Polymerisationsmethode hinsichtlich der Monomertypen und Reaktionsbedingungen. Deshalb gibt es in der Literatur seit ihrer Entdeckung eine rasant angestiegene Anzahl der Veröffentlichung über die RAFT-Polymerisation. Auf zwei neuere Reviews sei hier hingewiesen [20, 64].

Der RAFT-Prozess basiert auf zwei degenerativen Kettentransfergleichgewichten, die in Abbildung 3.1 illustriert sind. Im reversiblen Vorgleichgewicht kommt es über die Addition eines Radikals an das RAFT-Agens 1 zur Bildung eines intermediären RAFT-Radikals 2, welches sofort wieder zu den Edukten zurückfragmentieren kann. Dieses RAFT-Intermediat ist mit Hilfe der stabilisierenden Z-Gruppe in der Lage das ungepaarte Elektron zu stabilisieren. Es kann auch bei der Fragmentierung zur Freisetzung der Abgangsgruppe R, welche dann als Radikal R• vorliegt, und des Makro-RAFT-Agens 3 kommen. Das abgehende Radikal ist seinerseits in der Lage wieder an das RAFT-Agens zu addieren, oder durch Reinitiierung eine propagierende Polymerkette zu bilden.

Im Hauptgleichgewicht addiert ein Radikal reversibel an ein Makro-RAFTAgens. Über die Ausbildung des intermediären RAFT-Radikals 4 kommt es erneut zur Fragmentierung in ein Makro-RAFT-Agens und in ein wachsendes Radikal. Aufgrund der hohen Reaktionsgeschwindigkeit der Addition und Fragmentierung sind alle wachsenden Ketten an einem RAFT-Gleichgewicht beteiligt und liegen nicht 
lange in freier Radikalform vor. Die bekannteste und am häufigsten verwendete Stoffklasse des RAFT-Agens sind die Dithioester, die für eine große Anzahl von Monomeren effektiv einsetzbar sind. Ebenso eignen sich Trithiocarbonate (Kapitel 4) und Xanthate (Kapitel 5).

Für eine industrielle Anwendung der RAFT-Polymerisation, welche selbstverständlich ein zentrales Ziel für die Neuentwicklung einer Polymerisationsmethode ist, ist die effektive Trennung des RAFT-Agens vom Polymermaterial nach der Polymerisation essentiell. RAFT-Agens wird in einer typischen Prozedur mit einer Konzentration von 100 bis 1000 ppm eingesetzt und bleibt quantitativ als ein Teil des Makro-RAFT-Agens im Polymerprodukt. Besonders bei der Fokussierung auf hoch entwickelte Materialien kann diese schwefelhaltige Substanz die zukünftigen Anwendungen stark eingrenzen. Folgende Punkte können dabei unerwünscht sein:

- Die Dithioesterverbindungen sind potential giftig [65].

- Das Polymermaterial ist meist farbig, weil die Dithioestergruppe sichtbares Licht relativ stark absorbiert [66].

- Das Material kann wegen des Schwefelgehalts unangenehme Gerüche besitzen bzw. entwickeln.

- Die reaktive Dithioestergruppe ist die physikalische Schwachstelle des Polymers bezüglich chemischer Zersetzungsprozesse.

Um diese Limitierungen aufzuheben, erscheint es lohnend, eine neue Technik zu entwickeln, mit welcher das RAFT-Agens nach der Polymerisation effektiv vom Polymermaterial abgetrennt werden kann und die Wiedergewinnung des wertvollen RAFT-Agens ermöglicht.

Die Verwendung eines heterogenen Katalysators ist wegen der einfachen Trennung und Wiedergewinnung ein seit langem bekannter Ansatz. Es ist nicht verwunderlich, dass bei über $90 \%$ der chemischen Reaktionen in der Industrie heterogene Katalysatoren großtechnisch eingesetzt werden. Systeme von an Festkörper gebundenen Katalysatoren für kontrollierte Polymerisation wurden z. B. für NMP [47, 48, 49] und ATRP [50, 51, 52] vorgestellt. Die meisten Anwendungen des Kontrollagens, das mit einer chemischen Bindung an der Oberfläche eines festen Substrat gebunden ist, 
fokussiert sich auf die Entwicklung von dichten Polymerbürsten [53, 54], und erstaunlich wenig Aufmerksamkeit wird auf die Anwendung der kontrollierten radikalischen Polymerisation mit gebundenem Kontrollagens gerichtet. Berichte über die RAFTPolymerisation an Oberflächen sind spärlich $[55,56]$ und beginnen erst seit kurzem Interesse zu erwecken, da die Zeitspanne seit der Erfindung der RAFT-Technik relativ kurz ist.

Drei unterschiedliche Wege sind für die Durchführung der RAFT-Polymerisation an Oberflächen vorstellbar:

1. Radikalbildung an der Oberfläche: Das initiierende Radikal wird direkt an der Oberfläche gebildet, z. B. durch einen an der Oberfläche gebundenen Initiator [67] oder durch strahleninduzierte Radikalbildung [68]. Bei der Ausführung dieser Methode gehen wichtige Vorteile der RAFT-Polymerisation verloren. Das an der Oberfläche gebundene Polymer stellt nur den Anteil der Ketten dar, der von dem kontinuierlichen Initiierungsprozess stammt und der deshalb keine kontrollierte Molmasse und keinerlei Mikroarchitektur besitzt.

2. Immobilisierung über die R-Gruppe: Das RAFT-Agens wird an der Oberfläche eines festen Substrats über seine reinitiierende Abgangsgruppe (R-Gruppe) gebunden $[69,70]$. Durch die Immobilisierung der R-Gruppe befindet sich das ungepaarte Elektron immer an dem Kettenende des gebundenen Makroradikals, während die RAFT-Gruppe sich von der Oberfläche entfernen. Dieser Weg erlaubt eine einfache Ausbildung einer dichten Polymerbürste, weil sich die aktive RAFT-Gruppe außerhalb des entstehenden Polymersfilm befindet und deshalb für ein propagierendes Radikal leicht zugänglich ist.

3. Immobilisierung über die Z-Gruppe: Das RAFT-Agens wird an der Oberfläche über seine stabilisierende Gruppe (Z-Gruppe) chemisch fixiert [71, 72]. Das propagierende Radikal bleibt dann solange ungebunden in der flüssigen Phase, bis es die RAFT-Gruppe an der Oberfläche erreicht. Dieser Methode ist wegen der schlechten Zugänglichkeit der aktiven RAFT-Gruppe weniger geeignet für die Herstellung einer dichten Polymersbürste, weil die aktive RAFT-Gruppe sehr nah an der Oberfläche ist und deshalb von der progressiv wachsenden Polymerkette abgeschirmt wird. Ähnliche Effekte werden bei der Herstellung von 
Sternpolymer mit multifunktionellen RAFT-Agenzien beobachtet, bei denen die Z-Gruppe den Kern bilden [73, 74, 75]. Um die Polymerisation dennoch gut kontrollieren zu können, kann zusätzlich zu dem gebundenen RAFT-Agens ein freies RAFT-Agens hinzu gegeben werden. Dieses freie RAFT-Agens soll helfen, die konventionelle Polymerisation in der Lösung zu kontrollieren.

Für den Fall, dass eine niedrige Pfropfdichte des RAFT-Agens vorliegt, kann die aktive RAFT-Gruppe dennoch für ein propagierendes Radikal erreichbar sein. Damit ist eine lebende Polymerisation an der Oberfläche mit der effektiven Kontrolle der Molmasse möglich. Die Tatsache, dass der schwefelhaltige Teil des gebundenen Polymers sich nicht von der Oberfläche entfernen kann, bildet die Voraussetzung für eine effektive Trennung und Wiedergewinnung des RAFT-Agens von dem polymeren Produkt.

Das Ziel dieser Arbeit ist das Potential der RAFT-Polymerisation, bei der das RAFT-Agens an der Oberfläche über seine Z-Gruppe kovalent gebunden wird, zu erforschen. Für dieses Ziel wird die Substanzpolymerisation von Styrol bzw. MMA mit Cumyldithiobenzoat als RAFT-Agens durchgeführt, das über die stabilisierende Phenylgruppe an der Oberfläche von Silica-Nanopartikeln chemisch fixiert ist.

Die Strategie zur Herstellung eines schwefelfreien, wohldefinierten Polymers sowie zur Trennung und Wiedergewinnung des RAFT-Agens ist in Abbildung 3.2 dargestellt. Das an der Silica-Oberfläche verankerte Cumyldithiobenzoat (8, Abbildung 3.2.a) wird einer radikalischen Polymerisation zugegeben, um die Reaktion zu kontrollieren. Abbildung 3.2.b zeigt das entstehende Polymer, das über die Dithioester an die Nanopartikel angebunden ist und das die reinitiierende Cumylgruppe als Kettenende hat. Dieses Oberflächenpolymer wird in diesem Kapitel auch als die „lebende“ Fraktion des Polymerisationssystems bezeichnet, weil diese Fraktion die reaktive Dithioestergruppe enthält und deshalb zum weiteren Kettenwachstum fähig ist (vergleiche mit Spezies 3 in Abbildung 3.1).

Das Polymer, das aus der irreversiblen Terminierungsreaktion zwischen zwei Radikalen entsteht, wird in diesem Kapitel als „,totes“ Polymer bezeichnet, weil es keine reaktive Gruppe trägt und keinen Schwefel enthält; sein Kettenwachstum ist unwiederbringlich beendet. Dieses Polymer befindet sich ausschließlich in der flüssigen Phase und ist deshalb perfekt von dem lebenden Material durch einfaches Filtrie- 


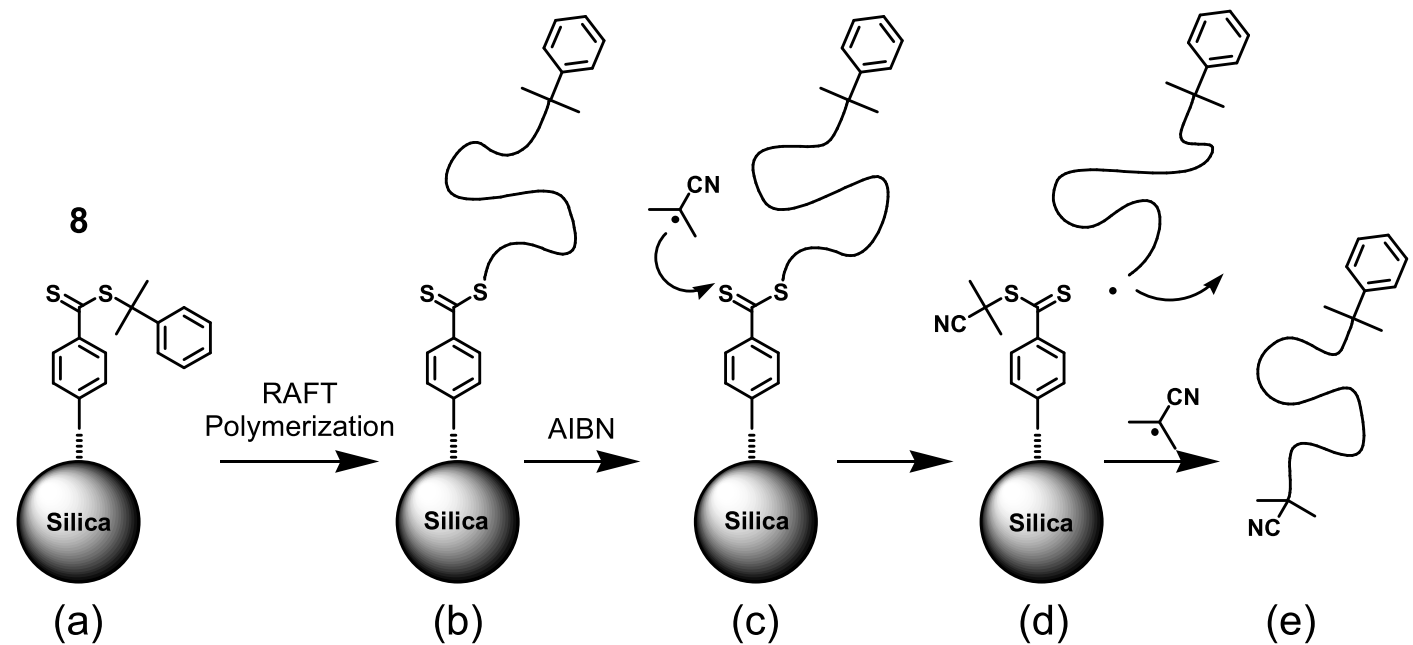

Abbildung 3.2: Allgemeiner Weg für die Bildung des an der Oberfläche gebundenen Polymers mittels der Immobilisierung der Z-Gruppe von Cumyldithiobenzoat und die anschließende Abspaltung des Polymers von Silica-Nanopartikel.

ren zu trennen. Die Fraktion des toten Polymers ist in einer effizienten RAFTPolymerisation im Vergleich zu dem lebenden Material relativ klein. Sie steigt jedoch mit zunehmendem Monomerumsatz wegen der Akkumulation des terminierenden Nebenprodukts an.

Abbildung 3.2.c zeigt, wie das angebundene lebende Polymer von einem kleinen Radikal, z. B. von einem aus AIBN stammenden Cyanoisopropylradikal, in Abwesenheit des Monomers angegriffen wird. Das Radikal setzt durch einen einfachen Addition-Fragmentation-Kettentransfer-Reaktionsschritt ein Makroradikal frei, das seinerseits mit einem anderen Primärradikal von AIBN terminiert (Abbildung 3.2.d). Als Terminierungsprodukt entsteht ein schwefelfreies Polymer, das bei einer Beteiligung des kleinen Radikals unabhängig von dem Terminierungsmodus die gleiche Molmassenverteilung wie die des lebenden Polymers hat (Abbildung 3.2.e). Terminierungsreaktionen zwischen zwei Makroradikalen kann bei dieser Abspaltungstechnik vernachlässigt werden, weil ein großer Überschuss an Cyanoisopropylradikalen vorliegt.

Die Verwendung von Silica scheint sehr gut für industrielle Anwendungen geeignet zu sein, weil das Silica chemisch resistent, mechanisch-stabil und kostengünstig 
ist. Eine detaillierte Erforschung der RAFT-Polymerisation an Silica-Oberfläche erscheint interessant und vielversprechend. In diesem Kapitel werden die Verwendung von Silica-Nanopartikel als Substrat für den gebundenen Cumyldithioester und seine Fähigkeit, die Molmasse in der Polymerisation von Styrol bzw. MMA zu kontrollieren, untersucht. Der Einfluss der Partikelkonzentration und der RAFT-AgensBeladungsdichte auf der Oberfläche wird studiert. Die Polymerisation wird ohne Verwendung eines freien RAFT-Agens durchgeführt, um das RAFT-Agens anschließend von dem Polymermaterial zu trennen, es wiederzugewinnen und ein schwefelfreies Polymerendprodukt zu erhalten.

\subsection{Experiment}

\subsubsection{Synthese von gebundenem Cumyldithiobenzoat}

Die Synthese von an Silica gebundenem Cumyldithiobenzoat basiert sowohl auf der in der Literatur beschriebenen Synthese von Cumyldithiobenzoat [76] als auch auf der Immobilisierungsmethode von Methoxysilane auf der Silica-Oberfläche [77]. Dabei sind im Prinzip drei unterschiedliche Syntheserouten vorstellbar, die in der Abbildung 3.3 dargestellt sind.

In den ersten beiden Synthesewegen wird die Vorstufe des RAFT-Agens mit der reaktiven Methoxysilangruppe zuerst an der Silica-Oberfläche gebunden. Erst danach wird die gebundene Vorstufe zum Dithiobenzoat umfunktioniert. Beide Syntheserouten haben den Nachteil, dass die bei der Reaktion auftretenden Verunreinigungen oder die unumgesetzten Edukte auch an der Silica-Oberfläche haften bleiben und dass diese unerwünschten gebundenen Substanzen die anschließende RAFTPolymerisation stören können.

Der beste Weg, um die Silica-Oberfläche mit dem RAFT-Agens ohne Verunreinigung zu modifizieren, ist scheinbar die dritte Syntheseroute. Das dabei entstehende RAFT-Agens hat reaktive Methoxysilangruppen, die an der Silica-Oberfläche chemisch binden können. Trotz großer Anstrengungen konnte diese Syntheseroute für die Immobilisierung von Dithiobenzoat nicht verwirklicht werden ${ }^{1}$, da die Methoxy-

\footnotetext{
${ }^{1}$ Die Immobilisierung von Trithiocarbonat verläuft hingegen erfolgreich mit dieser dritten Syntheseroute, was im nächsten Kapitel beschrieben wird.
} 


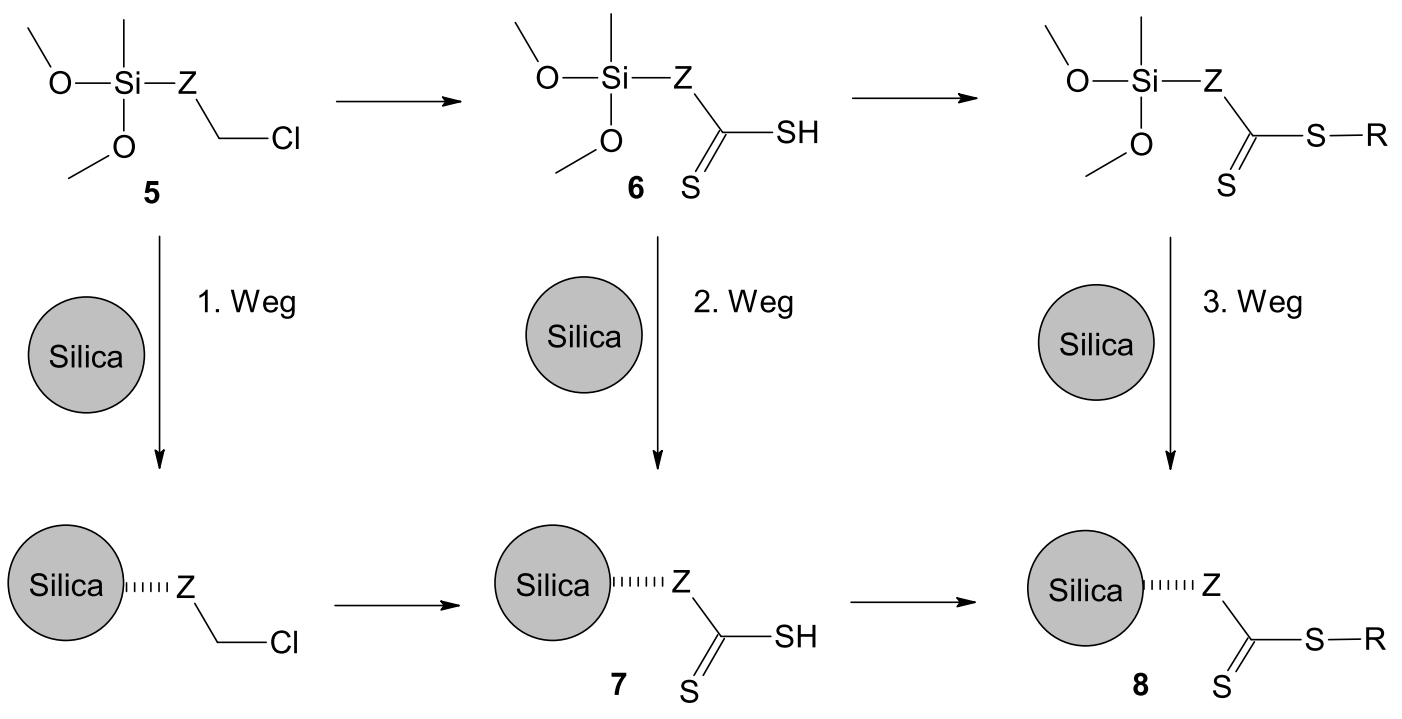

Abbildung 3.3: Drei unterschiedliche Wege zur Synthese von an Silica gebundenem Cumyldithiobenzoat.

silangruppen sehr reaktiv sind und während der Synthese schnell zu den unlöslichen Siloxane kondensieren.

Abbildung 3.4 stellt die erfolgreich durchgeführte Synthese von an Silica gebundenem Cumyldithiobenzoat über den zweiten Weg detailliert dar. Die Verwendung der Methyldimethoxysilangruppe als Verankerung ist gegenüber der Trimethoxysilangruppe vorteilhafter, denn die Methyldimethoxysilangruppe ist weniger reaktiv und kondensiert entsprechend viel langsamer. Als Zwischenstufe erhält man die immobilisierte Dithiobenzoesäure 7, welche auch ein effektives RAFT-Agens ist (siehe Kapitel 6).

\section{Synthese von gebundener Dithiobenzoesäure $\mathbf{7}$}

$\mathrm{Zu}$ einer 30\%igen Lösung von Natriummethanolat (1.6 g, $8.6 \mathrm{mmol}$ ) in Methanol $(10 \mathrm{~mL})$ wurden elementarer Schwefel $(0.29 \mathrm{~g}, 8.6 \mathrm{mmol})$ und tropfenweise (Chlormethylphenylethyl)-methyldimethoxysilan (1.2 g, $4.3 \mathrm{mmol}$, Gemisch aus m,pIsomere) hinzugegeben. Die erhaltene braune Lösung wurde über Nacht unter Rückfluss erhitzt. Nachdem die Lösung auf Raumtemperatur abgekühlt war, wurde der weiße Feststoff (Natriumchlorid) abfiltriert und Methanol unter vermindertem Druck entfernt. Den verbleibenden braunen Feststoff löste man in 50 mL destilliertem Was- 


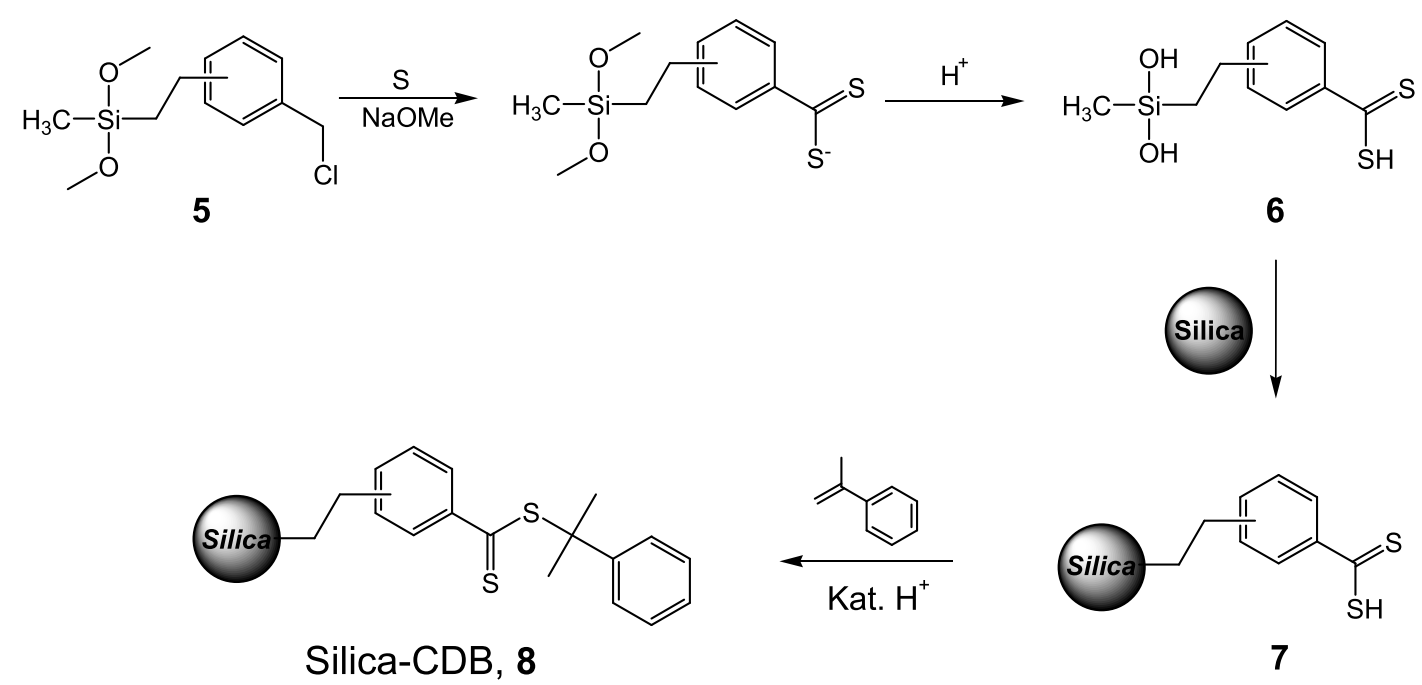

Abbildung 3.4: Syntheseroute von Cumyldithiobenzoat, das über die Z-Gruppe an der Oberfläche von Silica-Nanopartikel chemisch gebunden ist.

ser und extrahierte diese Lösung dreimal mit jeweils 20 mL Diethylether. Bei der Zugabe der letzten Volumina Diethylether $(240 \mathrm{~mL})$ wurde das Zwei-Phasen-Gemisch mit konzentrierte Salzsäure angesäuert. Die wässrige Phase verlor dabei ihre charakteristische braune Farbe, gleichzeitig färbte sich die etherische Phase violett. Der Farbumschlag deutete auf die Bildung einer Dithiobenzoesäure 6, die eine aktive bifunktionelle Silanol-Gruppe trägt.

Danach wurde $10 \mathrm{~mL}$ von der $240 \mathrm{~mL}$ violetten Lösung in einer Suspension aus nanometergrößen Silicapartikeln (9.0 g für eine RAFT-Agens-Dichte von rund $10 \mathrm{mmol}$ pro Kilogramm Silica) und Diethylether hergestellt. Die Suspension wurde bei Zimmertemperatur eine Stunde lang gerührt. Nach dem Entfernen des Diethylether unter vermindertem Druck wurde die an Silica gebundene Dithiobenzoesäure (Silica-DTBA, 7) in einer Soxhlet-Apparatur mit Dichlormethan 12 Stunden lang gewaschen, um die physikalisch gebundenen Verunreinigungen zu entfernen. Da sowohl Silica-DTBA als auch die Verunreinigung eine intensive Farbe hatten, konnte man den Verlauf dieser Reinigung einfach verfolgen, indem man die Farbe von Dichlormethan beobachtete. Das Endprodukt, ein leicht violettes Pulver, wurde unter Vakuum bei Zimmertemperatur getrocknet. 
Synthese von gebundenem Cumyldithiobenzoat $\mathbf{8}$

Die erhaltene Silica-DTBA 7, $\alpha$-Methylstyrol (2.4 g, $20 \mathrm{mmol}$ ) und eine katalytische Menge von $p$-Toluolsulfonsäure wurden in $50 \mathrm{~mL}$ Toluol gegeben. Man rührt die Suspension über Nacht bei Zimmertemperatur. Nach dem Entfernen des Toluols unter verminderten Druck erhält man das an Silica-Oberfläche gebundene Cumyldithiobenzoat 8. Die Reinigung des modifizierten Silica erfolgte wie bei Silica-DTBA 7 in einer Soxhlet-Apparatur mit Dichlormethan.

\subsubsection{Polymerisation}

Das Monomer wurde mittels dreimaligem „freeze-pump-and-thaw" an einer Hochvakuumpumpe (Edwards, EXC 120) entgast. Die Lösung wurde in eine mit Argon befüllte Handschuhbox (Braun, Lab Master 130) eingeschleust und darin mit AIBN (ca. $5 \mathrm{mmol} \cdot \mathrm{L}^{-1}$ ) vermischt. Die Lösung wurde dann in Schraubdeckelgläser, die unterschiedliche Menge von Silica-CDB enthielt, aufgeteilt. Die Schraubdeckelgläser wurden mit den entsprechenden gasdichten Deckeln in der Handschuhbox verschlossen.

Die Polymerisation wurde bei Umgebungsdruck in einem temperierten Ölbad bei $60{ }^{\circ} \mathrm{C}$ durchgeführt. Die Reaktionslösung wurde nur bei der Suspension mit einer niedrigen Konzentration von Silica-CDB gerührt, aber nicht bei hoher Partikelkonzentration von $m($ Silica-CDB $): m($ Monomer $)=37: 100$, weil die Suspension zu zähflüssig war. Nach den angegebenen Zeiten wurden die Schraubdeckelgläser aus dem Ölbad entfernt und zum Reaktionsabbruch in ein Eisbad gestellt.

Zur gravimetrischen Monomerumsatzbestimmung wurde nach erfolgter Polymerisation das Monomer bei Zimmertemperatur unter vermindertem Druck entfernt. Die Mischung aus Polymer und festem Substrat wurde in einer SoxhletApparatur mit Dichlormethan 12 Stunden lang gewaschen, um die restliche AIBNMenge vollständig zu entfernen und um das freie Polymer abzutrennen. Die Molmassenverteilung des toten Polymers wurde mittels Gelpermeationschromatographie (GPC) bestimmt. Das Silica, das das gebundene Polymer trägt (siehe Abbildung 3.2.b), wurde in Vakuum bei Zimmertemperatur getrocknet. 


\subsubsection{Abspaltung des gebundenen Polymers}

In einer mit Argon befüllten Handschuhbox wurde das mit lebenden Polymerketten bedeckte Silica in eine Lösung von $0.5 \mathrm{~mol} \cdot \mathrm{L}^{-1}$ AIBN in Toluol gegeben, die vorher mittels dreimaligem "freeze-pump-and-thaw" entgast wurde. Die Suspension wurde bei $80^{\circ} \mathrm{C}$ für drei Stunden erhitzt. Nach der Polymerisation wurden die Silicapartikel abfiltriert und das Filtrat wurde mittels GPC analysiert (siehe Abbildung 3.2.e).

\subsection{Ergebnisse und Diskussion}

\subsubsection{Charakterisierung von Silica-CDB}

Die Konzentration des gebundenen Dithiobenzoats, die für eine effiziente Regulierung der Menge des RAFT-Agens in dem Polymerisationssystem bekannt sein muss, wird durch eine semiquantitative Methode bestimmt. Die experimentelle Ausbeute von 6 beträgt ca. $50 \%$ bezüglich der eingesetzten Mengen von 5. Die anschließende Verknüpfung von 6 an der Oberfläche von Silica verläuft vollständig, weil die intensive violette Farbe des Diethylether nach der Reaktion komplett verschwindet. Für den letzten Reaktionsschritt von 7 zum Endprodukt 8 wird ebenfalls eine quantitative Ausbeute angenommen. Dies wird durch die Charakterisierung mit RamanSpektroskopie, einer geeigneten Technik für die Analyse der an Silica gebundenen organische Verbindungen [78], bestätigt.

Das Raman-Spektrum von Silica-CDB, das in Abbildung 3.5 dargestellt ist, zeigt eindeutig die Schwingungsbande von Aryl- und Alkyl-C-H-Bindungen um $3000 \mathrm{~cm}^{-1}$, die Bande der aromatischen C-C-Schwingung bei $1450 \mathrm{~cm}^{-1}$ und die Gerüstschwingungsbande des aromatischen Ringes zwischen 800 und $1000 \mathrm{~cm}^{-1}$. Diese Raman-Schwingungsbanden stimmen mit der Erwartung für an Silica gebundenes Cumyldithiobenzoat sehr gut überein. Die Schwingung der S-H-Gruppe von dem nicht umgewandelten Zwischenprodukt 7 würde ein deutliches Signal um $2600 \mathrm{~cm}^{-1}$ verursachen, weil die S-H-Gruppe eine besonders starke Raman-Schwingungsbande in diesem Bereich hat [79]. Es ist beim Betrachten der Abbildung 3.5 offensichtlich, dass es keinerlei Raman-Aktivität in dem Bereich um $2600 \mathrm{~cm}^{-1}$ gibt. Dieser Befund weist auf eine vollständige Umwandlung der Dithiobenzoesäure in das Endprodukt 


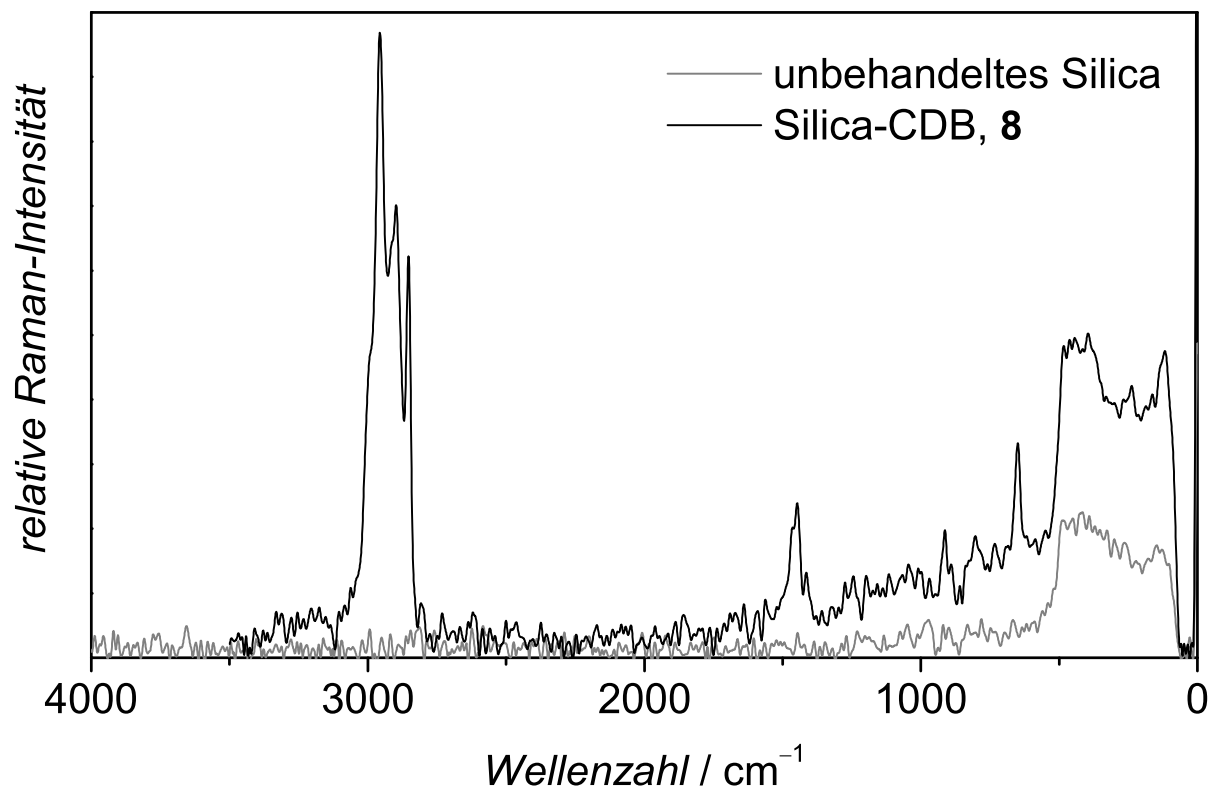

Abbildung 3.5: Raman-Spektrum des unbehandelten Silica und des am Silica gebundenen Cumyldithiobenzoats.

8 hin. Zusammenfassend beträgt die Ausbeute des Endproduktes ca. $50 \%$ bezüglich der eingesetzten Menge des Eduktes 5. Dieses Ergebnis ermöglicht eine Abschätzung der Belegungsdichte des RAFT-Agens auf der Silica-Oberfläche.

\subsubsection{Simulation der RAFT-Polymerisation}

Um die Polymerisation zu charakterisieren, die von an Silica gebundenem Cumyldithiobenzoat kontrolliert wird, wird das freie Polymer zuerst analysiert. Es entsteht aus der irreversiblen Terminierungsreaktion zwischen zwei propagierenden Radikalen, ist frei in der flüssigen Phase beweglich und deshalb relativ einfach durch Filtration abzutrennen. Die erste Beobachtung ist, dass das freie Polymer eine weiße Farbe besitzt. Das Fehlen einer starken UV/vis-Absorbanz des toten Polymermaterials beweist die Abwesenheit einer Dithiogruppe, welche typischerweise farbig ist. Dieser Befund stimmt mit der Annahme überein, dass sich nur totes Polymer in der flüssigen Phase befindet, während das lebende Polymer an der Silica-Oberfläche fest gebunden ist. 


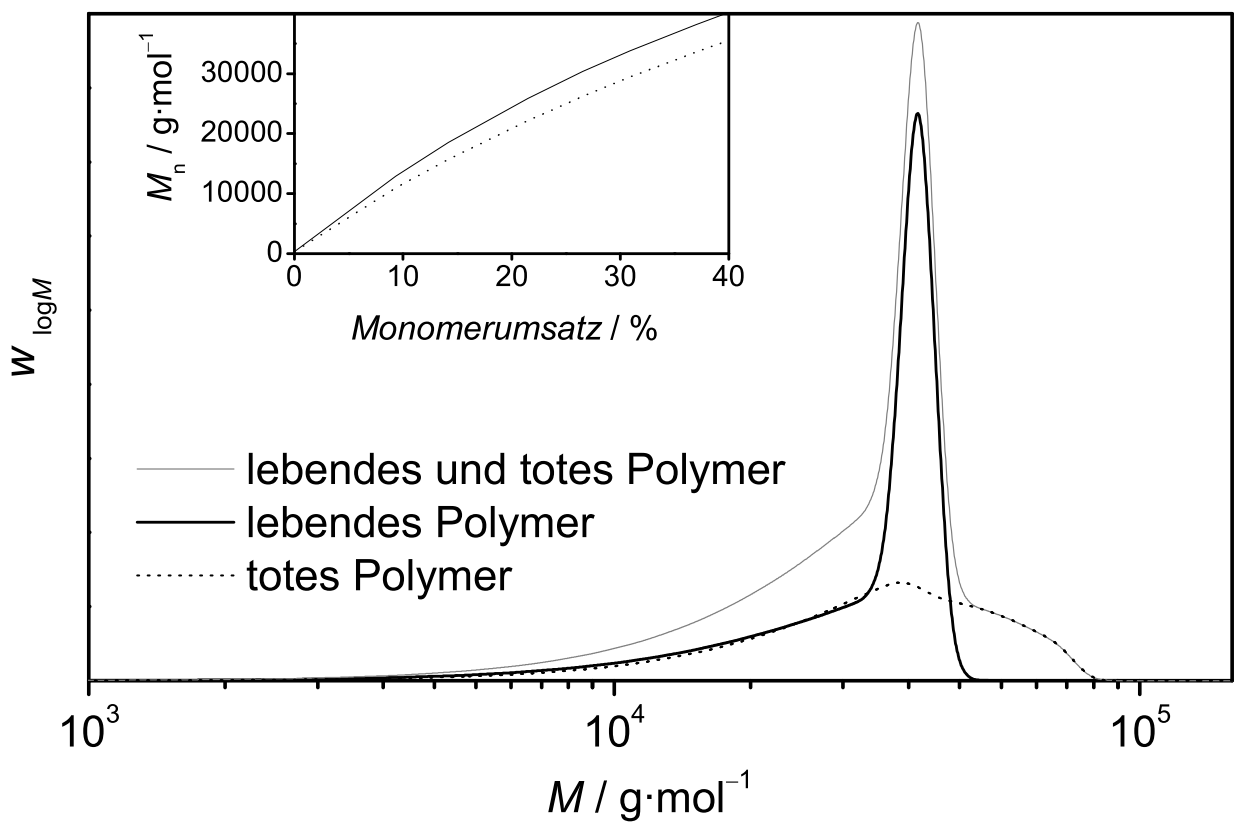

Abbildung 3.6: Simulierte Molmassenverteilungen und die Entwicklung der mittleren Molmasse $M_{\mathrm{n}}$ mit dem Monomerumsatz von lebendem und totem Polymer, das aus der Polymerisation von Styrol mit einer RAFT-Agens-Konzentration von $10^{-2} \mathrm{~mol} \cdot \mathrm{L}^{-1}$ bei einer Reaktionszeit von 10 Stunden entsteht. Die Substanzpolymerisation wird von $10^{-2} \mathrm{~mol} \cdot \mathrm{L}^{-1}$ AIBN bei $60{ }^{\circ} \mathrm{C}$ initiiert. Das kinetische Modell und die dazugehörigen Geschwindigkeitskoeffizienten sind in [81] beschrieben.

Das tote Polymer kann näherungsweise als ein Indikator für den gesamten Polymerisationsprozess benutzt werden. Diese Annäherung wird verständlich bei Betrachtung der Abbildung 3.6. Die Abbildung zeigt eine simulierte Molmassenverteilung des aus einer RAFT-Polymerisation entstehenden Polymers, zusätzlich sind die Molmassenverteilungen sowohl vom lebenden als auch vom toten Polymer getrennt dargestellt. Die Simulation wird mit Hilfe des Programmpakets PREDICI ${ }^{\circledR}$ und des in der Literatur beschriebenen kinetischen Modells durchgeführt [80, 81].

Das tote Polymer (gepunktete Linie in Abbildung 3.6) zeigt eine breitere Molmassenverteilung als das lebende Polymer (fette durchgezogene Linie), was in der Tatsache begründet ist, dass das tote Polymer durch Terminierung von zwei Radikalen während des ganzen Polymerisationsprozesses kontinuierlich produziert wird. Es 
enthält also alle möglichen Kettenlängen vom Primärradikal bis zur engverteilten Kettenlänge des lebenden Polymers. Für den Fall, dass die Terminierungsreaktion ausschließlich über Radikalkombination stattfindet, weist das tote Polymer auch Kettenlängen auf, die doppelt so groß sind wie die des lebenden Polymers. Diesen Effekt kann man bei höheren Molmassen in Abbildung 3.6 gut erkennen.

Der Gewichtsmittelwert des Molekulargewichts ${ }^{2} M_{\mathrm{w}}$ des toten Polymers ist gröBer als $M_{\mathrm{w}}$ des lebenden Polymers, während der Zahlenmittelwert des Molekulargewichts $M_{\mathrm{n}}$ des toten Polymers etwas kleiner ist als $M_{\mathrm{n}}$ des lebenden Polymers. Der Polydispersitätsindex ist definiert als $P D I=M_{\mathrm{w}} / M_{\mathrm{n}}$. Daraus kann man auch sehen, dass das tote Polymer eine größere Polydispersität bzw. eine breitere Molmassenverteilung hat als das lebende Polymer. Diesen Sachverhalt kann man an der kleinen Auftragung in Abbildung 3.6 erkennen, wo die simulierte Entwicklung von $M_{\mathrm{n}}$ des toten und lebenden Polymers gegen den Monomerumsatz aufgetragen ist.

Es ist also offensichtlich, dass die $M_{\mathrm{n}}$-Werte des toten Polymers direkt mit den $M_{\mathrm{n}}$-Werten des lebenden Polymers zusammenhängen und deshalb als ein geeigneter Indikator für die Entwicklung der mittleren Molmasse während der RAFTPolymerisation dienen kann. Das tote Polymer enthält den schwefelfreien Teil des gesamten Polymermaterials. Seine mittlere Kettenlänge wächst mit steigendem Monomerumsatz, obwohl seine Molmassenverteilung relativ breit ist.

\subsubsection{Einfluss der Partikelkonzentration und der Belegungs- dichte des RAFT-Agens}

Abbildung 3.7 zeigt die experimentellen Molmassenverteilungen des freien Polystyrols, das sich nach der heterogenen RAFT-Polymerisation mit verschiedenen Konzentrationen von Silica-CDB bei $60{ }^{\circ} \mathrm{C}$ in der flüssigen Phase befindet. Der Monomerumsatz beträgt nach vier Stunden Reaktionszeit ca. $27 \%$ bei einer AIBNKonzentration von $5 \mathrm{mmol} \cdot \mathrm{L}^{-1}$. Die Silica-Nanopartikel haben eine Ladungsdichte an Cumyldithiobenzoat von $210 \mathrm{mmol}$ pro Kilogramm Silica. Diese Ladungsdichte, die den in dieser Arbeit maximal erreichten Wert darstellt, entspricht eine Oberflächenkonzentration von 0.32 RAFT-Agensmolekülen pro $\mathrm{nm}^{2}$, was rund $7 \%$ der in der Literatur berichteten Silanol-Beladungsdichte von 4.6 Hydroxyl-Gruppe pro

\footnotetext{
${ }^{2} M_{\mathrm{w}}$ wird hauptsächlich von der höheren Molmassenfraktion bestimmt
} 


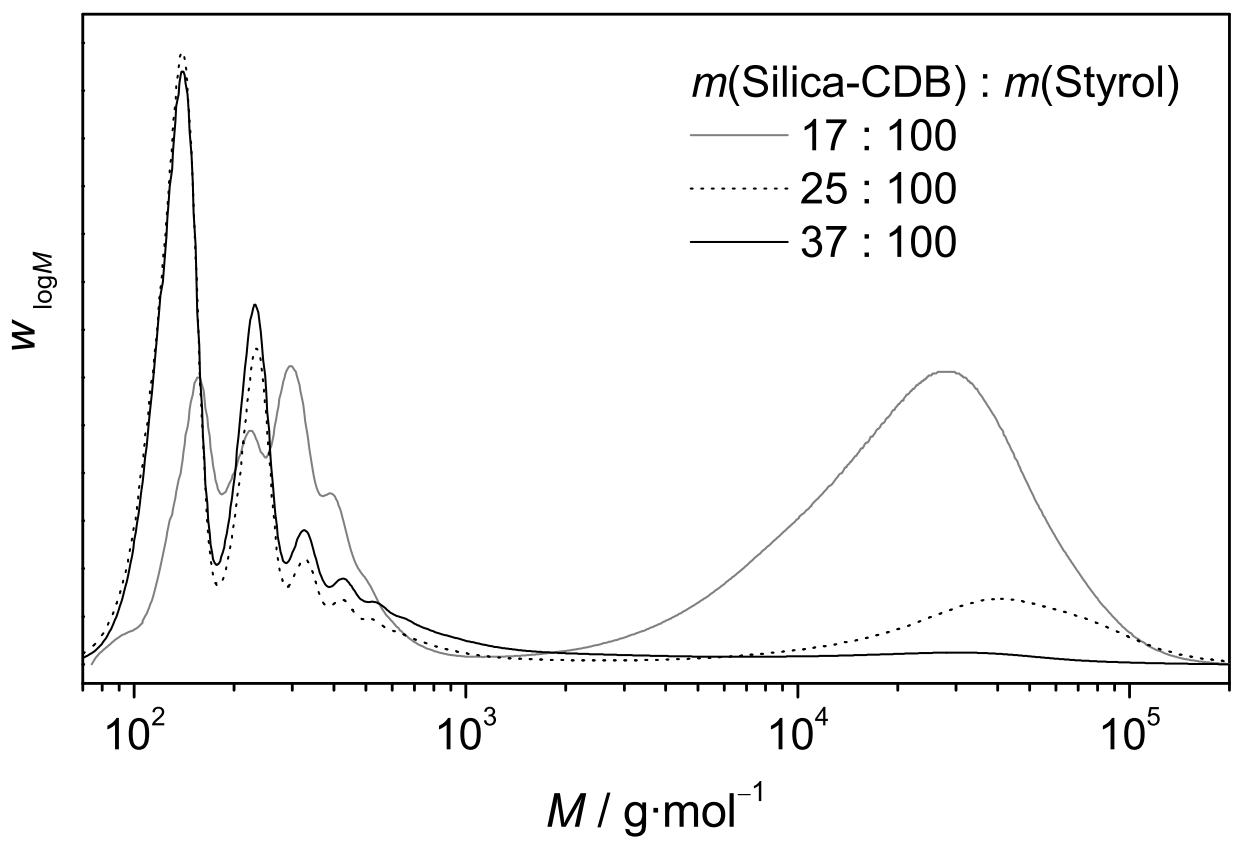

Abbildung 3.7: Molmassenverteilungen des freien Polymers, das in der flüssigen Phase bei der Substanzpolymerisation von Styrol mit verschiedenen Konzentrationen von Silica-CDB bei $60{ }^{\circ} \mathrm{C}$ gebildet wird. Die Silica-Nanopartikel haben eine Ladungsdichte an Cumyldithiobenzoat von $210 \mathrm{mmol}$ pro Kilogramm Silica. Die Verteilungen sind auf die Menge des Oligomermaterials unterhalb von $1000 \mathrm{~g} \cdot \mathrm{mol}^{-1}$ normiert.

$\mathrm{nm}^{2}$ der Silica-Oberfläche ausmacht [82].

Das freie Polymer, das bei der Verwendung dieser hohen CDB-Ladungsdichte entsteht, zeigt eine bimodale Molmassenverteilung, die in eine Oligomer-Region unterhalb von $1000 \mathrm{~g} \cdot \mathrm{mol}^{-1}$ und in eine Polymer-Region oberhalb $10000 \mathrm{~g} \cdot \mathrm{mol}^{-1}$ eingeteilt werden kann (siehe Abbildung 3.7). Die Multimodalität in der Oligomer-Region kommt von der effizienten GPC-Trennung von einzelnen Oligomer-Spezies. Das Polymer, das im Gesamten eine bimodale Molmassenverteilung hat, weist stark darauf hin, dass zwei getrennte Polymerisationsprozesse im System nebeneinander stattfinden:

1. Das Oligomermaterial stammt aus der Polymerisationsaktivität in der Nähe der Silica-Oberfläche, wo das gebundene RAFT-Agens in hoher Konzentration vorliegt und seine starke Transferaktivität die Molmasse effektiv limitiert. 
2. In der flüssigen Phase, fernab von der Silica-Oberfläche, ist das RAFT-Agens nicht verfügbar. Hier findet ein konventioneller radikalischer Polymerisationsprozess statt. Daraus entsteht das Polymer mit hoher Kettenlänge und relativ breiter Molmassenverteilung.

Diese Hypothese wird durch Betrachtung der Abbildung 3.7 bestätigt: Die Materialmenge aus der konventionellen Polymerisation geht zurück, wenn die Konzentration der Silica-CDB-Partikel erhöht wird, wobei sich das Verhältnis zwischen der flüssigen Phase und der Oberfläche der Silica-CDB-Partikel verkleinert. Gleichzeitig wird die mittlere Molmasse des Oligomermaterials, das in der Nähe der Oberfläche des modifizierten Silica gebildet wird, signifikant kleiner. Dieser Befund wird verständlich, wenn man die Berechnung der theoretischen Molmasse $M_{\mathrm{n}}^{\text {theo }}$ betrachtet.

$$
M_{\mathrm{n}}^{\text {theo }}=\frac{[\mathrm{M}]_{0} \cdot M W_{\text {Monomer }} \cdot U_{\mathrm{M}}}{[\mathrm{RAFT}]_{0}+d \cdot f \cdot\left([\mathrm{I}]_{0}-[\mathrm{I}]_{U}\right)} \approx \frac{[\mathrm{M}]_{0} \cdot M W_{\text {Monomer }} \cdot U_{\mathrm{M}}}{[\mathrm{RAFT}]_{0}}
$$

In dieser Gleichung sind $M_{\mathrm{n}}^{\text {theo }}$ die theoretische Molmasse, $M W_{\text {Monomer }}$ die Molmasse des Monomers, $[\mathrm{RAFT}]_{0}$ die Startkonzentration des RAFT-Agens, $d$ spiegelt den Terminierungsmodus wider, $f$ die Initiatoreffektivität, $[\mathrm{I}]_{0}$ die Startkonzentration des Initiators und $[\mathrm{I}]_{\mathrm{U}}$ die Initiatorkonzentration beim Monomerumsatz $U$. Da der Initiator langsam zerfällt und seine Konzentration relativ gering ist, wird dessen Beitrag zur Berechnung der theoretischen Molmasse zur Vereinfachung vernachlässigt.

Aus der Gleichung 3.1 wird deutlich, dass eine hohe (lokale) RAFT-AgensKonzentration ein niedermolekulares Material ergibt. Die Verwendung einer hohen Konzentration an Silicapartikel mit einer hohen Belegungsdichte von Cumyldithiobenzoat verhindert zwar die unerwünschte konventionelle Polymerisation in der flüssigen Phase, aber sie begrenzt auch die Kettenlänge des entstehenden Polymers, weil die Gesamtkonzentration des RAFT-Agens im System sehr hoch ist.

Bei Betrachtung der Abbildung 3.7 ist nun verständlich, dass die Gesamtkonzentration des RAFT-Agens im System beim gleichzeitigen Erhalt des vorteilhaften kleinen Verhältnisses zwischen der flüssigen Phase und der Oberfläche der Silicapartikel reduziert werden muss, um ein Polymermaterial mit hoher und kontrollierter Molmasse zu erhalten. 
Dieses Gleichgewicht zwischen der Partikel-Konzentration und der Belegungsdichte des RAFT-Agens ist optimal, wenn die Partikel-Konzentration mindestens 0.3 g Silica-CDB pro Gramm Monomer beträgt und wenn die maximale Belegungsdichte des RAFT-Agens um eine Größenordnung verringert wird. Solch eine Systemanordnung ergibt eine ausreichende Homogenität des gebundenen RAFT-Agens über das ganze System und minimiert die Schwierigkeit der unterschiedlichen lokalen RAFT-Agens-Konzentration. Durch das Verwenden von Silica-CDB mit einer geringeren Beladungsdichte, wird die gesamte RAFT-Agens-Konzentration entsprechend verringert, sodass eine Bildung von hochmolekularem Polymermaterial nach Gleichung 3.1 ermöglicht ist. Außerdem wird die Zugänglichkeit der DithiobenzoatGruppe nahe an der Oberfläche durch eine geringere Beladungsdichte des RAFTAgens deutlich verbessert, wie in der Einleitung bereits diskutiert wurde. Damit sind alle denkbaren äußeren Bedingungen für eine kontrollierte heterogene RAFTPolymerisation erfüllt.

\subsubsection{Molmassen-Entwicklung des toten Polymers mit dem Monomerumsatz}

Wie erwartet, hat das tote Polymer in der flüssigen Phase eine unimodale Molmassenverteilung, wenn Silica-CDB mit einer Beladungsdichte des RAFT-Agens von 10 bzw. 18 mmol pro Kilogramm Silica verwendet wird und wenn eine Partikelkonzentration von rund $m$ (Silica-CDB) $: m$ (Monomer $)=37: 100$ eingestellt ist.

Die Auftragung der $M_{\mathrm{n}}$-Werte des toten Polymers gegen den Monomerumsatz ergibt zwei Hauptbeobachtungen.

1. In der Substanzpolymerisation von Styrol (Abbildung 3.8) und Methylmethacrylat (Abbildung 3.9) steigt die mittlere Molmasse mit dem Monomerumsatz an. Diese typische Molmassen-Entwicklung weist auf eine kontrollierte Polymerisation hin, die an der Oberfläche von Silica-CDB stattfindet. Die mittlere Molmasse des toten Polymers steigt parallel zu der des lebenden Polymers (vergleiche mit der kleinen Auftragung in Abbildung 3.6).

2. Abgesehen von experimenteller Streuung der Daten, die zum Teil von der inhomogenen Reaktionsbedingungen stammt, zeigen die mittleren Molmassen des 


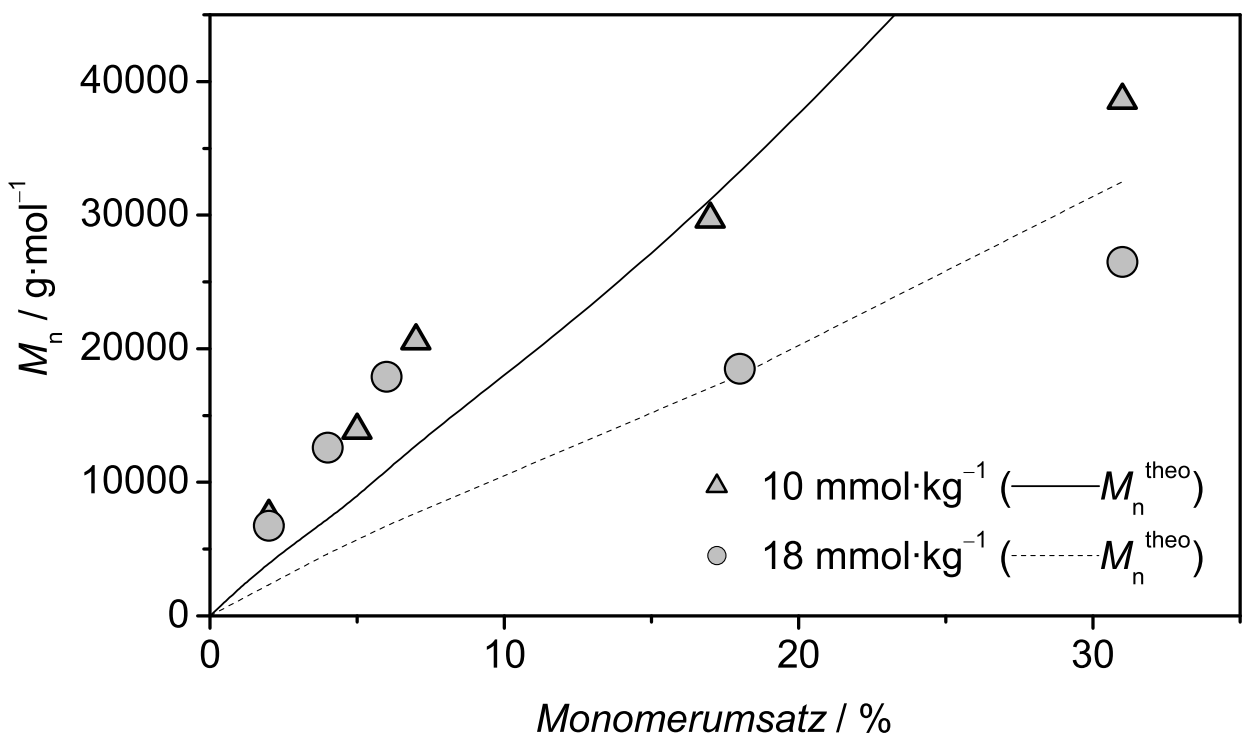

Abbildung 3.8: Auftragung des Zahlenmittels der Molmassen $M_{\mathrm{n}}$ des toten Polymers gegen den Monomerumsatz für die Polymerisation von Styrol bei $60{ }^{\circ} \mathrm{C}$, initiiert durch AIBN $\left(5.0 \cdot 10^{-3} \mathrm{~mol} \cdot \mathrm{L}^{-1}\right)$ und kontrolliert von Silica-CDB mit unterschiedlicher Beladungsdichte und einer Partikelkonzentration von $m$ (Silica-CDB) $: m$ (Monomer $)=37: 100$. Die Linien stellen die theoretischen Molmasse $M_{\mathrm{n}}^{\text {theo }}$ dar.

Polymers, das in dem System mit einer kleineren Beladungsdichte des RAFTAgens gebildet wird, eindeutig höhere Werte. Dieser Befund stimmt qualitativ mit der Gleichung 3.1 überein.

Diese zwei Beobachtungen erlauben die Schlussfolgerung, dass das an SilicaOberfläche gebundene Cumyldithiobenzoat die Polymerisation effektiv kontrolliert.

Beim Vergleich der experimentellen Molmasse des toten Polymers, die entsprechend der Simulation ähnlich wie die des lebenden Polymers sein soll, mit den nach Gleichung 3.1 berechneten theoretischen Werten $M_{\mathrm{n}}^{\text {theo }}$ wird eine relativ gute Übereinstimmung für die Styrol-Polymerisation gefunden, was auf eine sehr gute Kontrolle von Silica-CDB hinweist.

Abbildung 3.9 zeigt jedoch signifikante Unterschiede zwischen den experimentellen und den theoretischen Werten von der MMA-Polymerisation; die theoretischen Werte sind doppelt bis dreimal größer als die experimentellen Molmassen. Obwohl 


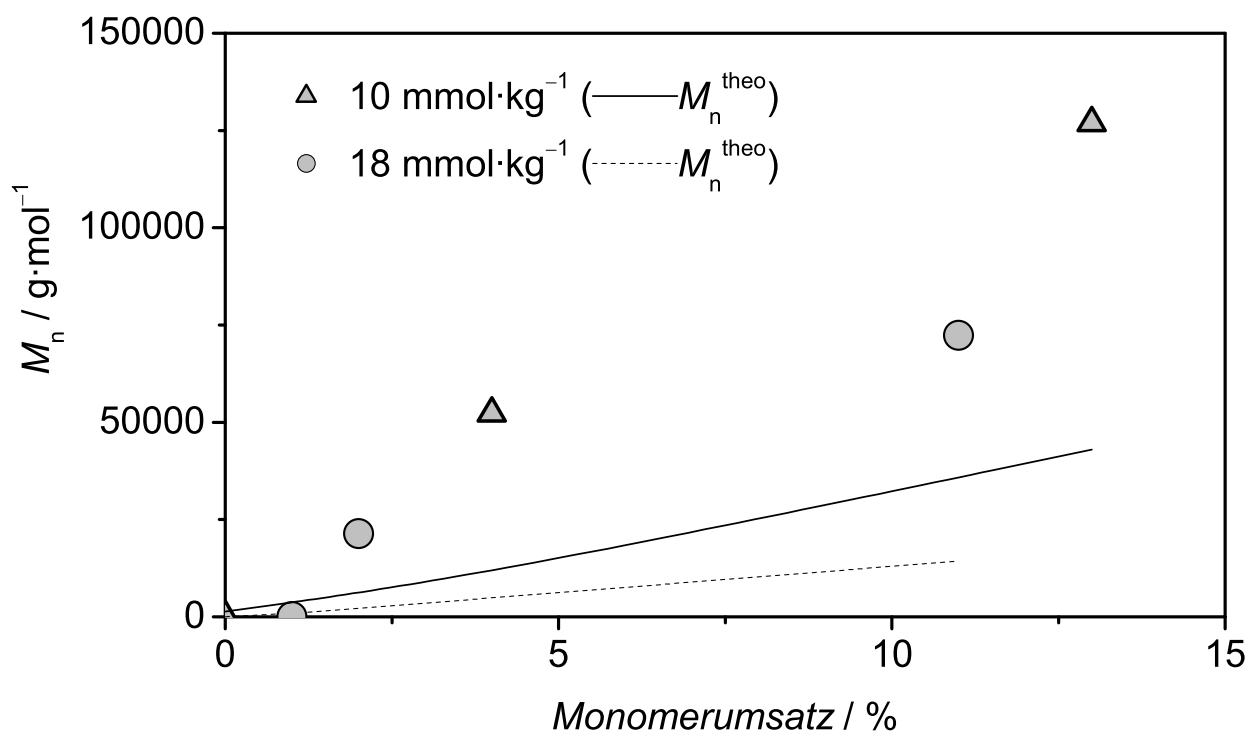

Abbildung 3.9: Auftragung des Zahlenmittels der Molmassen $M_{\mathrm{n}}$ des toten Polymers gegen den Monomerumsatz für die Polymerisation von Methylmethacrylat bei $60{ }^{\circ} \mathrm{C}$, initiiert durch AIBN $\left(5.0 \cdot 10^{-3} \mathrm{~mol} \cdot \mathrm{L}^{-1}\right)$ und kontrolliert von Silica-CDB mit unterschiedlicher Beladungsdichte und einer Partikelkonzentration von $m$ (Silica-CDB) : $m$ (Monomer) $=$ 37 : 100. Die Linien stellen die theoretischen Molmasse $M_{\mathrm{n}}^{\text {theo }}$ dar.

der Fehler der theoretischen Molmasse relativ hoch ist, weil die Gesamtkonzentration des RAFT-Agens mit Hilfe der semiquantitativ bestimmten Belegungsdichte von Cumyldithiobenzoat berechnet wird, zeigt diese signifikante Abweichung eine weniger effektive Molmassenkontrolle in der MMA-Polymerisation an. Geringe Effektivität und experimentelle Molmassen, die höher als die theoretischen Molmassen sind, treten oft in der MMA-Polymerisation auf und gründen hauptsächlich auf der tertiären Natur des propagierenden Radikals. Dadurch werden eine rasche Bildung des Makro-RAFT-Agens in der Anfangsphase und ein schnelles RAFT-Gleichgewicht verhindert. Eine detaillierte Diskussion dieses Effektes findet sich in [64].

\subsubsection{Abspaltung und Analyse des lebenden Polymers}

Im anschließenden Schritt wird das an Silica gebundene lebende Polymer entsprechend der oben beschriebenen Prozedur mit aus AIBN gebildeten Radikalen von der 


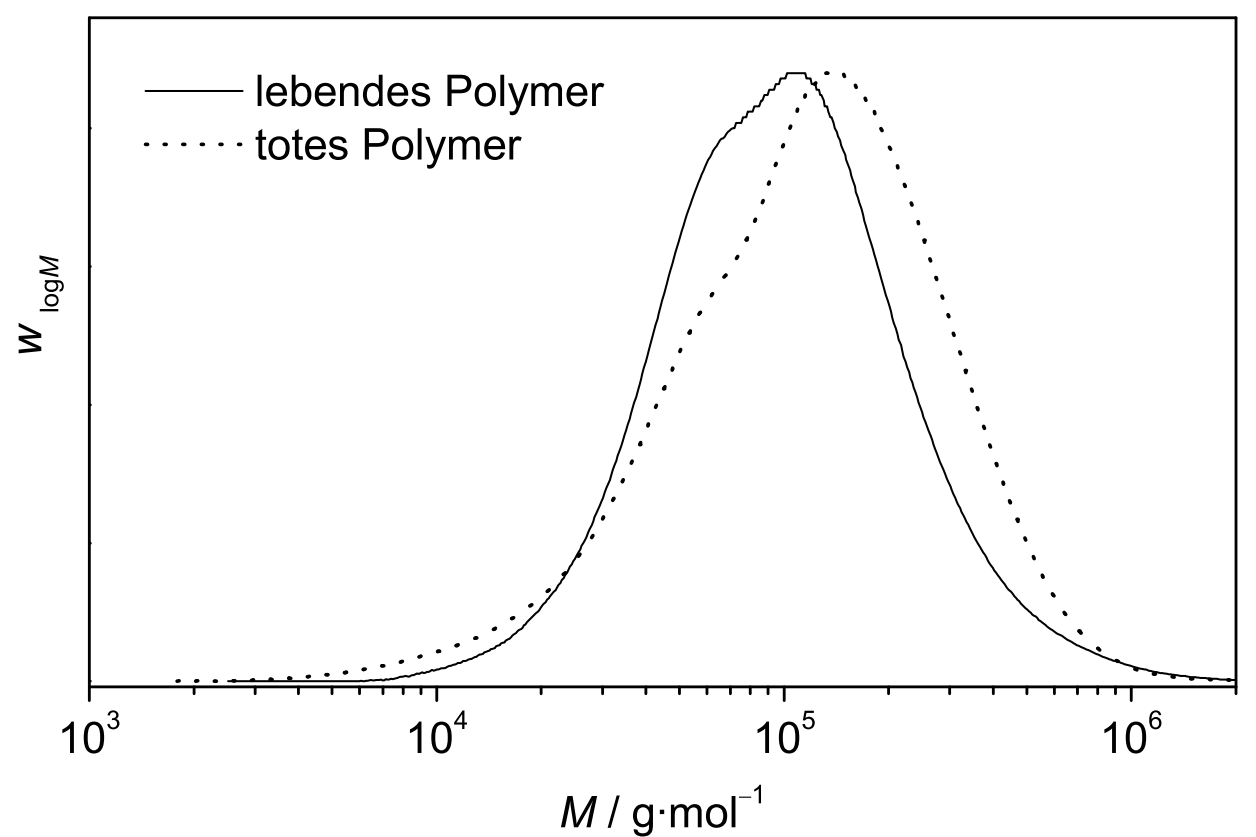

Abbildung 3.10: Molmassenverteilung des Polymers, das nach einem Monomerumsatz von $11 \%$ in der MMA-Polymerisation bei $60{ }^{\circ} \mathrm{C}$ entsteht. Die Polymerisation wird von AIBN $\left(5.0 \cdot 10^{-3} \mathrm{~mol} \cdot \mathrm{L}^{-1}\right)$ initiiert und von Silica-CDB mit einer Beladungsdichte von $18 \mathrm{mmol}$ pro Kilogramm und einer Partikelkonzentration von $m$ (Silica-CDB) : $m$ (Monomer) $=$ 37 : 100 kontrolliert. Die gepunktete Linie zeigt das tote Polymer, das in der flüssigen Phase gebildet wird. Die durchgezogene Linie gehört zu dem lebenden Polymer, das nach der Polymerisation von der Silica-Oberfläche abgespalten wird.

Oberfläche abgespalten. Das erhaltene Polymer ist farblos und hat keinerlei UV/visAbsorbanz, was entsprechend der in Abbildung 3.2.e gezeigten Situation auf das Fehlen einer Dithiobenzoat-Gruppe hinweist. Das erhaltene Silica hat wieder die Farbe blassrosa und zeigt ein ähnliches Raman-Spektrum wie das von Silica-CDB. Diese Beobachtung beweist, dass das RAFT-Agens auf der Silica-Oberfläche immer noch festgebunden ist und dass die Wiedergewinnung des RAFT-Agens erfolgreich verlaufen ist.

Eine typische Molmassenverteilung des lebenden Polymers, das nach der Polymerisation vom Silica abgespalten wird, ist in Abbildung 3.10 zusammen mit der Verteilung des toten Polymers dargestellt. Zwei wichtige Unterschiede zwischen dem 
toten und lebenden Polymer können bei der Betrachtung ihrer Molmassenverteilung sofort erkannt werden:

1. Das an Oberfläche gebundene lebende Polymer hat eine engere Molmassenverteilung. In der Tat haben alle experimentell erhaltene, lebende Polymere deutlich kleinere Werte für die Polydispersität als die toten Polymere.

2. Das lebende Polymer hat einen kleineren Anteil an hochmolekularem Polymer, was zu einem kleineren Gewichtsmittelwert des Molekulargewichts $M_{\mathrm{w}}$ führt.

Diese Befunde stimmen mit den Unterschieden zwischen lebendem und totem Polymer aus der obigen Simulation der RAFT-Polymerisation voll überein (siehe Abbildung 3.6). In einer MMA-Polymerisation verläuft nur $40 \%$ der gesamten Terminierung über Kombination [83], die eine Bildung an toten Polymeren mit einer Kettenlänge bis zu den doppelten Wert des lebenden Polymers ermöglicht. Die Terminierung über Disproportionierung ergibt totes Polymer mit einer mittleren Kettenlänge, die der des lebenden Polymers gleicht. Leider können diese beiden Fraktionen bei GPC-Messungen nicht getrennt werden.

Die Menge des an der Silica-Oberfläche gebundenen lebenden Polymers beträgt etwa $50 \%$ des gesamten Polymermaterials. Basierend auf den Molmassenverteilungen des lebenden und toten Polymers können diese Massenprozent in Molprozent umgewandelt werden. Daraus ergibt sich, dass nach dem Ende der Polymerisation ca. $70 \%$ aller Polymerketten auf der Silica-Oberfläche lebend verbleiben. Dieser Wert ist viel höher als der in der Literatur beschriebene Wert, der weniger als $10 \%$ für das System mit mikrometergroßen Silicapartikeln beträgt [71]. Die Verkleinerung der Silicapartikeln auf die Nanometer-Skala hat folglich einen positiven Einfluss auf die Effektivität des Systems.

Es muss angemerkt werden, dass in einer idealen RAFT-Polymerisation der Anteil des lebenden Polymers von der RAFT-Agens-Konzentration und von der Menge an primären Radikalen vorgegeben wird. Der langsam zerfallende Initiator setzt kontinuierlich primäre Radikale frei, um die Polymerisation aufrecht zu erhalten. In einem nicht-idealen Fall, bei dem nicht alle verfügbaren Dithiobenzoat-Gruppen für ein propagierendes Radikal zugänglich sind, kann die Menge des lebenden Polymers reduziert werden. Beispielweise kann eine Dithiobenzoat-Gruppe innerhalb des Silicapartikels sitzen und deshalb nicht als Transferagens zur Verfügung stehen. Solche 
Effekte, die besonders bei der Fixierung des RAFT-Agens über die Z-Gruppe beobachtet werden, reduzieren ungünstigerweise das Verhältnis zwischen lebenden und toten Polymerketten.

Alle bisher beschriebene Beobachtungen stimmen gut mit den Erwartungen einer kontrollierten heterogenen RAFT-Polymerisation an Silica-Oberfläche überein. Die Polydispersität des lebenden Polymers, das von den Silicapartikeln abgespalten wurde, ist aber nicht kleiner als 1.5, was ein weiteres Indiz für eine effiziente RAFTPolymerisation wäre. Für eine lebende Polymerisation hat das lebende Polymer eine relativ breite Verteilung, seine Polydispersität beträgt zwischen 1.5 und 2.5.

Dieser Befund entkräftet nicht die Feststellung, dass eine RAFT-Polymerisation an der Oberfläche stattfindet. Es weist eher darauf hin, dass die Unzugänglichkeit der Dithiobenzoat-Gruppe so groß ist, dass eine signifikante Verbreiterung der Molmassenverteilung entsteht. Während der Zeitspanne, in der sich das propagierende Makroradikal von einer Dithiobenzoat-Gruppe zu einer anderen bewegt, addiert es in der flüssigen Phase mehrere Monomereinheiten in einer unkontrollierten Art und Weise, bis es wieder mit einer Dithiobenzoat-Gruppe reagiert und in eine schlafende Spezies umgewandelt wird. Diese flüssige Phase, wo keine Kontrolle der Molmasse stattfindet, wird zwar durch die Erhöhung der Konzentration von Silica-CDB minimiert, aber die gleiche Homogenität des RAFT-Agens wie in einem flüssigen System ist in diesem heterogenen System, bei dem das RAFT-Agens über die Stabilisierungsgruppe an der Oberfläche gebunden ist, grundsätzlich nicht erreichbar. Daher ist eine inhärente Verbreiterung der Molmassenverteilung unvermeidlich.

\subsubsection{An Silica gebundene Dithiobenzoesäure}

Es ist bekannt, dass die Dithiobenzoesäure (DTBA) in der Lage ist, die Polymerisation von Styrol, Methylmethacrylat und Methylacrylat zu kontrollieren [84, 85]. Das erhaltene Polymer hat eine kontrollierte Molmasse und eine niedrige Polydispersität unterhalb von 1.5. Die Effektivität der Dithiobenzoesäure ist beim Vergleich mit Cumyldithiobenzoat geringer. Trotzdem ist die Verwendung von DTBA als RAFTAgens sehr interessant, weil seine Herstellung einfach und, wegen der hohen Ausbeute, kostengünstig ist.

Im Rahmen dieser Arbeit wurde daher die RAFT-Polymerisation mit der Dithio- 


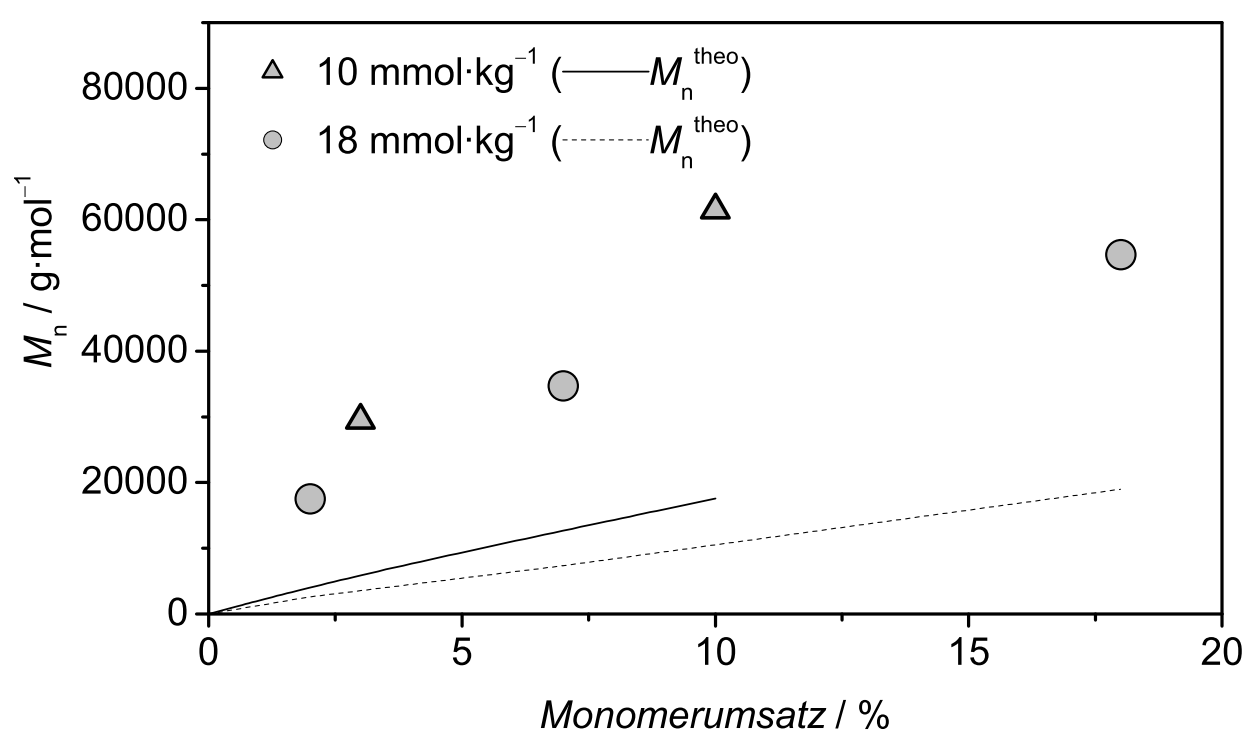

Abbildung 3.11: Auftragung des Zahlenmittels der Molmassen $M_{\mathrm{n}}$ des toten Polymers gegen den Monomerumsatz für die Polymerisation von Styrol bei $60{ }^{\circ} \mathrm{C}$, initiiert durch AIBN $\left(5.0 \cdot 10^{-3} \mathrm{~mol} \cdot \mathrm{L}^{-1}\right)$ und kontrolliert von Silica-DTBA 7 mit unterschiedlichen Beladungsdichten und einer Partikelkonzentration von $m$ (Silica-DTBA) : $m$ (Monomer) $=$ 37 : 100. Die Linien stellen die theoretischen Molmasse $M_{\mathrm{n}}^{\text {theo }}$ dar.

benzoesäure detailliert untersucht. Ein kinetischer Mechanismus dieser kontrollierten Polymerisation wird in Kapitel 6 vorgeschlagen. An dieser Stelle werden die Immobilisierung der Dithiobenzoesäure an der Silica-Oberfläche und ihre Fähigkeit zur Kontrolle der heterogenen RAFT-Polymerisation von Styrol bzw. MMA beschrieben.

Die an Silica gebundene Dithiobenzoesäure (Silica-DTBA, 7) ist die direkte Vorstufe des immobilisierten Cumyldithiobenzoats (Silica-CDB, 8). In der Tat ist die Herstellung von Silica-DTBA durch den Wegfall einer Synthesestufe einfacher und kostengünstiger.

Abbildung 3.11 zeigt die Entwicklung der mittleren Molmasse des toten Polymers mit dem Monomerumsatz in der heterogenen RAFT-Polymerisation mit SilicaDTBA. Das Polymer nimmt linear mit dem Umsatz zu. Außerdem ist die mittlere Molmasse bei höherer Beladungsdichte mit Dithiobenzoesäure auf der Oberfläche 


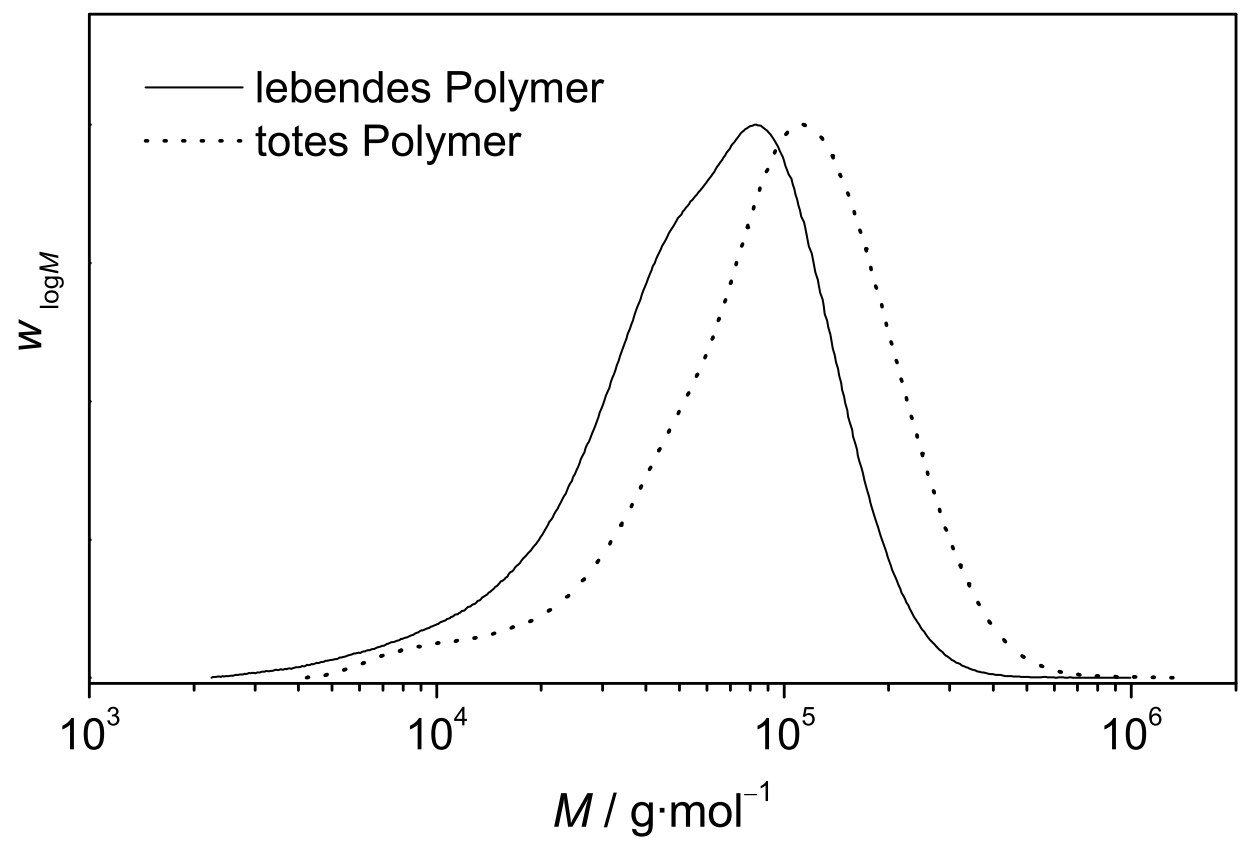

Abbildung 3.12: Molmassenverteilung des Polystyrols, das nach einem Monomerumsatz von $10 \%$ in der MMA-Polymerisation bei $60{ }^{\circ} \mathrm{C}$ gebildet wurde. Die Polymerisation wurde durch AIBN $\left(5.0 \cdot 10^{-3} \mathrm{~mol} \cdot \mathrm{L}^{-1}\right)$ initiiert und von Silica-DTBA 7 mit einer Beladungsdichte von $18 \mathrm{mmol}$ pro Kilogramm Silica und einer Partikelkonzentration von $m$ (SilicaDTBA ) $: m$ (Monomer) $=37: 100$ kontrolliert. Die gepunktete Linie zeigt das tote Polymer, das in der flüssigen Phase gebildet wurde. Die durchgezogene Linie gehört zu dem lebenden Polymer, das nach der Polymerisation von der Silica-Oberfläche abgespalten wurde.

entsprechend dem RAFT-Mechanismus kleiner als die Molmasse mit der niedrigeren Konzentration. All dies weist auf eine erfolgreiche heterogene RAFT-Polymerisation mit Silica-DTBA hin.

Abbildung 3.8 zeigt eine gute Übereinstimmung der theoretischen Molmasse mit den experimentellen Werten für die heterogene RAFT-Polymerisation von Styrol mit dem gebundenen Cumyldithiobenzoat (Silica-CDB). Dass die theoretische Molmasse bei der Polymerisation mit Silica-DTBA viel höher ist als die experimentellen Werte, weist auf die schlechte Effektivität der Dithiobenzoesäure hin. Eine detaillierte Untersuchung der RAFT-Polymerisation mit Dithiobenzoesäure ist in Kapitel 6 beschrieben. 
Das gebundene lebende Polymer an der Silica-Oberfläche kann durch Zugabe einer überschüssigen AIBN-Menge abgespalten werden. Abbildung 3.12 zeigt den Vergleich der Molmassenverteilung des toten und lebenden Polymers, das aus der heterogenen RAFT-Polymerisation mit Silica-DTBA entstanden ist. Die Molmassen von totem und lebendem Polymer sind entsprechend der obigen Simulation vergleichbar. Ihre Verteilungen sind relativ breit; die Gründe dafür sind bereits bei dem Silica-CDB diskutiert worden. Ihre Abweichung von einer Poisson-Verteilung, wie sie bei einer ideal kontrollierten RAFT-Polymerisation auftreten sollte, weist auf eine Inhomogenität der Polymerisationsmischung hin.

Um die heterogene RAFT-Polymerisation erfolgreich durchzuführen, müssen die äußeren Reaktionsbedingungen weiter optimiert werden. Außerdem sind weitere detaillierte Kenntnisse in der Dynamik der Polymerkette an der Oberfläche unbedingt notwendig.

Sowohl Silica-CDB als auch Silica-DTBA können die radikalische Polymerisation von Styrol und MMA kontrollieren. Da Silica-DTBA die Vorstufe von Silica-CDB ist, ist ihre Herstellung einfacher und kostengünstiger. Dieser Vorteil wird aber mit dem Nachteil erkauft, dass die gebundene Dithiobenzoesäure eine deutlich schlechtere Effektivität hat. Solange die Effektivität von Silica-DTBA nicht verbessert wird, scheint Silica-CDB für die Polymerisation von Styrol und MMA die bessere Wahl zu $\operatorname{sein}^{3}$.

\subsection{Schlussfolgerung}

Die radikalische Polymerisation von Styrol und Methylmethacrylat wird bei der Verwendung eines an der Silica-Oberfläche gebundenen RAFT-Agens bezüglich seiner Molmasse erfolgreich kontrolliert. Sowohl das Cumyldithiobenzoat als auch die Dithiobenzoesäure, die an der Oberfläche von Silica-Nanopartikeln über die stabilisierende Phenylgruppe kovalent gebunden sind, induzieren eine stetig steigende Kettenlänge bei einem zunehmenden Monomerumsatz. Die Methode der Immobilisierung des RAFT-Agens über die Z-Gruppe erlaubt die Herstellung von schwefelfreiem Poly-

\footnotetext{
${ }^{3}$ Eine vielversprechende Methode, die Effektivität von Dithiobenzoesäure zu verbessern, ist die Verwendung eines Initiator-Cocktails, welcher in Kapitel 6 beschrieben ist.
} 
mermaterial mit kontrollierter Molmasse und bietet einen Weg zur Wiedergewinnung des wertvollen RAFT-Agens. Zusätzlich ermöglicht die Fixierung des RAFT-Agens an Silicapartikeln eine effektive Trennung des lebenden Polymers von dem toten Polymer, das während der RAFT-Polymerisation gebildet wird. 


\section{Kapitel 4}

\section{An Silica gebundenes Trithiocarbonat}

Nachdem im vorhergehenden Kapitel die Immobilisierung von Dithioverbindungen an Silica-Nanopartikeln behandelt wurde, werden im Folgenden die Immobilisierung von Trithiocarbonaten und die zugehörigen heterogenen RAFT-Polymerisationen beschrieben.

\subsection{Einführung}

Zur Herstellung eines schwefelfreien Polymers mit kontrollierter Molmasse muss das RAFT-Agens von dem polymeren Produkt getrennt werden. Die generelle Strategie zur Trennung und Wiedergewinnung des RAFT-Agens wird in Abbildung $3.2 \mathrm{im}$ vorherigen Kapitel dargestellt und erläutert.

Ein Hauptziel dieser Arbeit ist es, die Einsatzmöglichkeit der heterogenen RAFTPolymerisation, bei der das RAFT-Agens an der Silica-Oberfläche über die stabilisierende Z-Gruppe kovalent gebunden wird, zu erforschen. Für dieses Ziel wird ein Syntheseweg für die Immobilisierung von Trithiocarbonaten an der Silica-Oberfläche gesucht. Zusätzlich werden die besten Bedingungen für eine erfolgreiche heterogene RAFT-Polymerisation ermittelt.

Die Trithiocarbonate, die zuerst von der CSIRO-Gruppe als RAFT-Agens eingesetzt wurden, eignen sich hervorragend für RAFT-Polymerisationen, weil sie auf eine Vielzahl unterschiedlichster Monomere erfolgreich angewendet werden können [44]. 
Die Immobilisierung von Trithiocarbonaten für die heterogene RAFT-Polymerisation wird in der Literatur nur von wenigen Forschungsgruppen berichtet $[72,86]$.

In diesem Kapitel wird die Immobilisierung von Trithiocarbonaten detailliert erläutert. Die heterogene RAFT-Polymerisation zur Herstellung von gebundenem Homopolymer und Blockcopolymer wird im Detail untersucht. Dabei soll der Schwerpunkt der Forschung der Mechanismus der RAFT-Polymerisation an der Oberfläche sein, da dieser bisher nur zum Teil beschrieben ist.

\section{$4.2 \quad$ Experiment}

\subsubsection{Synthese von an Silica gebundenem Trithiocarbonat}

Die Synthesestrategie für das an Silica gebundene Trithiocarbonat ist bereits anhand von Abbildung 3.3 im vorangehenden Kapitel beschrieben worden. Im Gegensatz zur Synthese vom immobilisierten Dithiobenzoat ist es in dieser Arbeit für Trithiocarbonat gelungen, ein RAFT-Agens mit einer Ankergruppe als Reinstoff zu isolieren. Damit ist der dritte und beste Weg zur Immobilisierung des RAFT-Agens in diesem Fall möglich. Die neu entwickelte Synthese von an Silica gebundenem Trithiocarbonat ist in der Abbildung 4.1 schematisch dargestellt.

Synthese von 3-(Trimethoxysilan)propyl-(phenylmethyl)-trithiocarbonat $\mathbf{2}$

$\mathrm{Zu}$ einer 30\%igen Lösung von Natriummethanolat (18.0 g, $0.10 \mathrm{~mol}$ ) in Methanol (30 mL) wurde tropfenweise 3-Mercaptopropyltrimethoxysilan 1 (19.6 g, 0.10 mol) unter Stickstoff bei $0{ }^{\circ} \mathrm{C}$ hinzugegeben. Die Reaktionslösung wurde für 2 Stunden bei Zimmertemperatur gerührt. Danach wurde Schwefelkohlenstoff (7.6 g, $0.10 \mathrm{~mol}) \mathrm{zu}$ der Reaktionslösung getropft. Nach 3 Stunden wurde Benzylchlorid (12.6 g, 0.10 mol) zu der Lösung unter Rühren zugegeben. Die Reaktionslösung wurde anschließend für 12 Stunden bei Zimmertemperatur gerührt.

Nach dem Entfernen des Lösungsmittels unter vermindertem Druck wurde das erwünschte Produkt mit Hilfe der Säulenchromatographie mit Silicagel gereinigt (Pentan : Dichlormethan $=9: 1 ; R_{\mathrm{f}}=0.47$ ). Man erhielt das Trimethoxysilanpropyl-(phenylmethyl)trithiocarbonat 2 als leicht gelbliches Öl (5.2 g, 0.014 mol, $14 \%)$. 

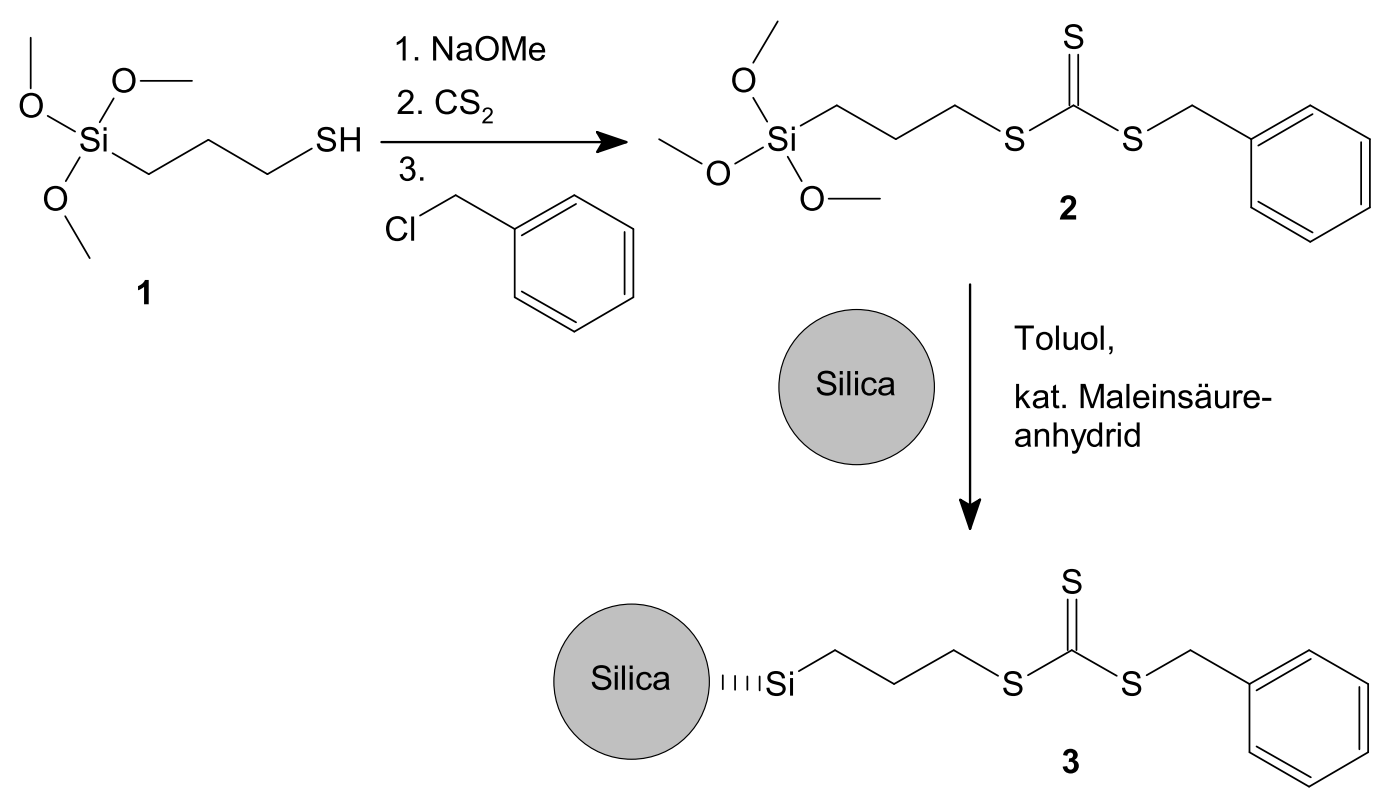

Abbildung 4.1: Syntheseroute vom via Z-Gruppe an der Silica-Oberfläche gebundenen Trithiocarbonat. Der Zebrastreifen zwischen Silica und Siliziumatom soll die chemische Verankerung von $\mathbf{3}$ an Silica-Oberfläche symbolisieren.

${ }^{1} \mathrm{H}-\mathrm{NMR}\left(300 \mathrm{MHz}, \mathrm{CDCl}_{3}\right), \delta(\mathrm{ppm}): 0.8\left(\mathrm{t}, 2 \mathrm{H},-\mathrm{CH}_{2}-\mathrm{Si}\right), 1.8\left(\mathrm{q}, 2 \mathrm{H},-\mathrm{CH}_{2}\right)$, $3.4\left(\mathrm{t}, 2 \mathrm{H},-\mathrm{CH}_{2}-\mathrm{S}\right), 3.6\left(\mathrm{~s}, 9 \mathrm{H}, 3 \times-\mathrm{CH}_{3}\right), 4.6\left(\mathrm{~s}, 2 \mathrm{H},-\mathrm{S}-\mathrm{CH}_{2}-\mathrm{Ph}\right), 7.2-7.4(\mathrm{~m}, 5 \mathrm{H}$, $\left.-\mathrm{C}_{6} H_{5}\right)$.

${ }^{13} \mathrm{C}-\mathrm{NMR}\left(300 \mathrm{MHz}, \mathrm{CDCl}_{3}\right), \delta(\mathrm{ppm}): 8.7\left(\mathrm{CH}_{2}-\mathrm{Si}\right), 21.6\left(\mathrm{CH}_{2}\right), 39.3\left(\mathrm{CH}_{2}-\mathrm{S}\right)$, $41.3\left(\mathrm{~S}-\mathrm{CH}_{2}-\mathrm{Ph}\right), 50.5\left(\mathrm{O}-\mathrm{CH}_{3}\right), 127.6,128,6,129,1,134.9\left(C_{6} \mathrm{H}_{5}\right), 223.4(C(=\mathrm{S}) \mathrm{S})$.

Elementaranalyse (\%): Kohlenstoff (45.8; ber. 46.4), Wasserstoff (6.1; ber. 6.1), Schwefel (28.3; ber. 26.5).

Synthese von an Silica gebundenem Trithiocarbonat $\mathbf{3}$ Das Trimethoxysilan-propyl-(phenylmethyl)trithiocarbonat 2 (0.12 g, $0.33 \mathrm{mmol})$ wurde in $10 \mathrm{~mL}$ Toluol gelöst und tropfenweise zu einer stark gerührten Suspension von nanometergroßen Silicapartikeln $(6.9 \mathrm{~g})$ in Toluol $(200 \mathrm{~mL})$ gegeben. Eine katalytische Menge von Maleinsäureanhydrid-Lösung (10 mg, $0.1 \mathrm{mmol}$, gelöst in $0.1 \mathrm{~mL}$ Wasser) wurde hinzugegeben. Die Reaktionsmischung wurde bei $60{ }^{\circ} \mathrm{C}$ für drei Stunden intensiv gerührt. 
Die erkaltete Suspension wurde filtriert. Das erhaltene modifizierte Silica wurde in einer Soxhlet-Apparatur mit Dichlormethan 12 Stunden lang gewaschen, um physikalisch gebundene Verunreinigungen zu entfernen. Anschließend wurde das Produkt unter Vakuum bei Zimmertemperatur über Nacht getrocknet.

Aus der Elementaranalyse wurde ein Schwefelmassenanteil von $0.4 \%$ bestimmt. Daraus wurde der Anteil des RAFT-Agens am modifizierten Silica zu $0.048 \mathrm{mmol} / \mathrm{g}$ berechnet. Basierend auf die vom Hersteller angegebene spezifische Oberfläche der nanometergroßen Silicapartikel $\left(400 \mathrm{~m}^{2} / \mathrm{g}\right)$ konnte eine Baladungsdichte an RAFTAgenzien auf der Silica-Oberfläche von 0.072 RAFT-Agens-Molekülen pro Quadratnanometer berechnet werden ${ }^{1}$.

\section{Synthese von Propyl-(phenylmethyl)-trithiocarbonat $\mathbf{4}$}

Die Synthese von 4 war mit der von 1 identisch, außer dass anstatt von 3-Mercaptopropyltrimethoxysilane Propanthiol (7.6 g, $0.10 \mathrm{~mol}$ ) eingesetzt wurde. Das erwünschte Produkt wurde mit Hilfe der Säulenchromatographie mit Silicagel gereinigt (Pentan; $\left.R_{\mathrm{f}}=0.17\right)$. Man erhält das Produkt 4 als leicht gelbliches Öl (19.0 g, $0.078 \mathrm{~mol}, 78 \%)$.

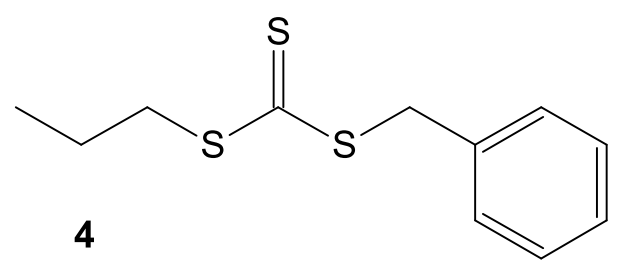

${ }^{1} \mathrm{H}-\mathrm{NMR}\left(300 \mathrm{MHz}, \mathrm{CDCl}_{3}\right), \delta(\mathrm{ppm}): 1.0$ (t, 3H, $\left.-\mathrm{CH}_{3}\right), 1.7$ (quint., $2 \mathrm{H},-\mathrm{CH}_{2}-$ $\left.\mathrm{CH}_{3}\right), 3.4\left(\mathrm{t}, 2 \mathrm{H},-\mathrm{CH}_{2}-\mathrm{CH}_{2}-\mathrm{CH}_{3}\right), 4.6\left(\mathrm{~s}, 2 \mathrm{H},-\mathrm{CH}_{2}\right), 7.2-7.4\left(\mathrm{~m}, 5 \mathrm{H},-\mathrm{C}_{6} H_{5}\right)$.

${ }^{13} \mathrm{C}-\mathrm{NMR}\left(300 \mathrm{MHz}, \mathrm{CDCl}_{3}\right), \delta(\mathrm{ppm}): 13.4\left(\mathrm{CH}_{2}-\mathrm{CH}_{2}-\mathrm{CH}_{3}\right), 21.5\left(\mathrm{CH}_{2}-\mathrm{CH}_{2}-\right.$ $\left.\mathrm{CH}_{3}\right), 38.8\left(\mathrm{CH}_{2}-\mathrm{CH}_{2}-\mathrm{CH}_{3}\right), 41.3\left(\mathrm{C}_{6} \mathrm{H}_{5}-\mathrm{CH}_{2}-\mathrm{S}\right), 127.7,128,6,129,2,135.0\left(C_{6} \mathrm{H}_{5}\right)$, $223.7(C(=\mathrm{S}) \mathrm{S})$.

\footnotetext{
${ }^{1}$ Die Beladungsdichte konnte mit dieser Methode bis zu 1.7 RAFT-Agens-Molekülen pro Quadratnanometer erhöht werden, indem die eingesetzte Menge von 2 entsprechend erhöht wurde.
} 


\subsubsection{Polymerisation}

Eine Lösung, bestehend aus Monomer (Methylacrylat oder Styrol) und Lösungsmittel (Toluol), wurde mittels dreimaligem "freeze-pump-and-thaw" an einer Hochvakuumpumpe (Edwards, EXC 120) entgast. Die Lösung wurde in eine mit Argon befüllte Handschuhbox (Braun, Lab Master 130) eingeschleust. Darin wurden AIBN als Initiator (ca. $1.2 \mathrm{mmol} \cdot \mathrm{L}^{-1}$ ) und gegebenenfalls 4 als freies ungebundenes RAFT-Agens vermischt. Die Lösung wurde dann in Schraubdeckelgläser, die eine unterschiedliche, genau eingewogene Menge Silica enthielten, aufgeteilt. Die Schraubdeckelgläser wurden mit entsprechenden gasundurchlässigen Teflon-Deckeln in der Handschuhbox verschlossen.

Die Polymerisation wurde bei Umgebungsdruck in einem temperierten Ölbad bei $60{ }^{\circ} \mathrm{C}$ unter intensiver Rührung durchgeführt. Nach den angegebenen Zeiten wurden die Schraubdeckelgläser aus dem Ölbad entfernt und zum Reaktionsabbruch in ein Eisbad gestellt. Die Polymerisationslösung wurde unter Vakuum gesetzt, um das restliche Monomer und Toluol zu entfernen. Der Monomerumsatz wurde gravimetrisch bestimmt.

Die entstehende Feststoffmischung wurde in einer Soxhlet-Apparatur mit Dichlormethan 12 Stunden lang gewaschen, um restliches AIBN und freies Polymer von dem modifizierten Silica zu trennen. Die Molmassenverteilung des freien Polymers erfolgte mittels Gelpermeationschromatographie (GPC). Das modifizierte Silica, das das gebundene Polymethylacrylat trägt, wurde nach dem Trocknen in Vakuum zur weiteren Modifikation und Analyse verwendet.

\subsubsection{Blockcopolymerisation}

Die Synthesemethode zur Herstellung von gebundenem Blockcopolymers ist schematisch in Abbildung 4.2 dargestellt. Die Feststoffmischung, die aus der heterogenen RAFT-Polymerisation mit an Silica gebundenem Trithiocarbonat nach dem Entfernen des restlichen Monomers und Toluol entstanden ist, wurde in die Handschuhbox eingeschleust. Sie enthielt eine definierte Menge an freiem und an Silica gebundenem Makro-RAFT-Agens. Eine Lösung, bestehend aus dem zweiten Monomer und Toluol, wurde mittels dreimaligem „freeze-pump-and-thaw“ an einer Hochvakuumpumpe entgast und auch in die Handschuhbox eingeschleust. Darin wurden die Feststoffmi- 


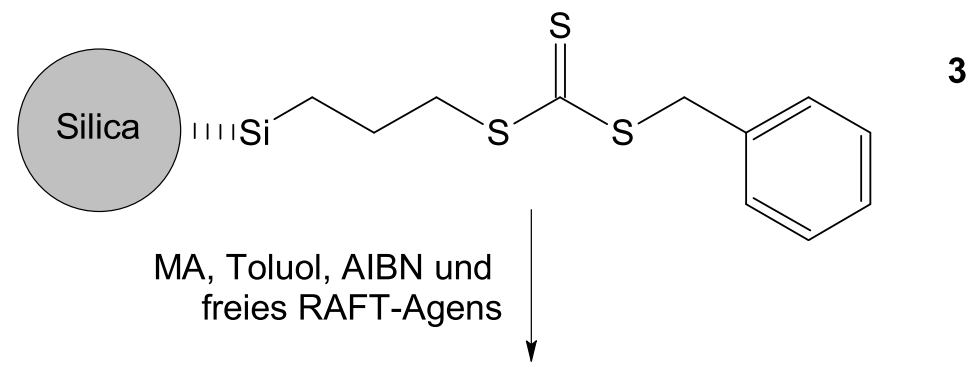

3
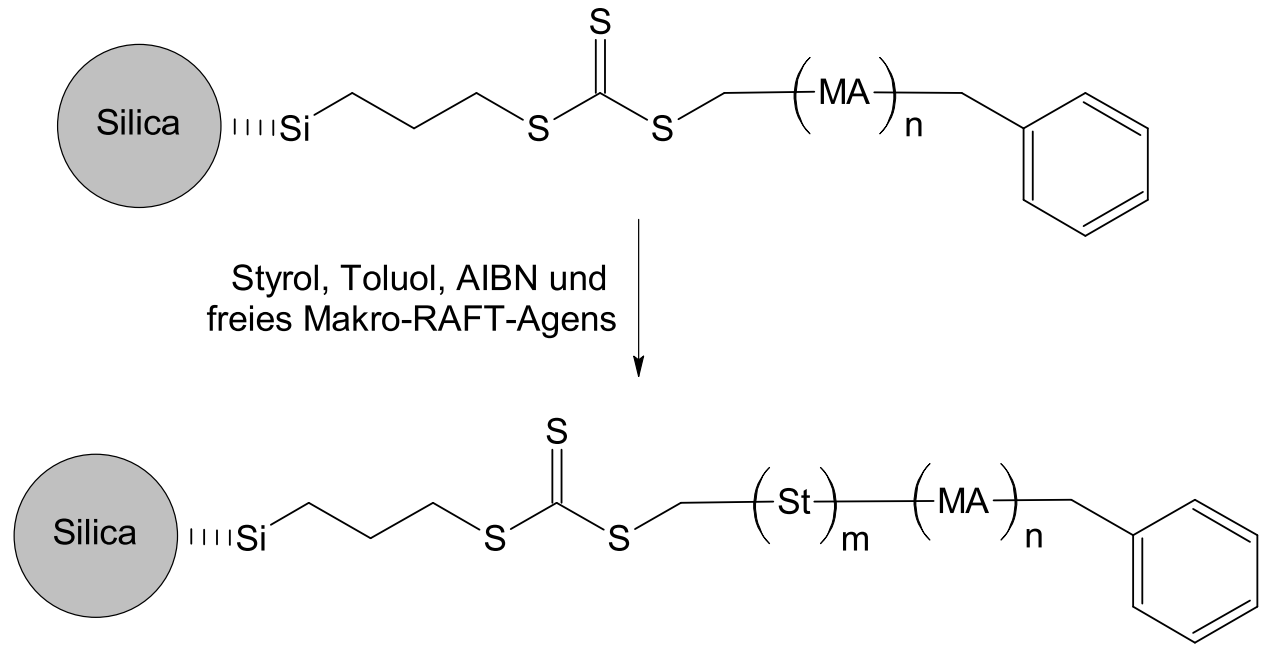

Abbildung 4.2: Synthesemethode für die Herstellung des Homo- und Blockcopolymer durch heterogene RAFT-Polymerisation mit dem gebundenen Trithiocarbonat.

schung, AIBN (ca. $1.2 \mathrm{mmol} \cdot \mathrm{L}^{-1}$ ) und die Reaktionslösung miteinander vermischt. Die Suspension wurde dann in Schraubdeckelgläser, die eine definierte Masse von modifiziertem Silica enthielten, aufgeteilt. Die Schraubdeckelgläser wurden mit den entsprechenden gasdichten Teflon-Deckeln in der Handschuhbox verschlossen.

Die anschließenden Polymerisationen, Aufarbeitungen und Charakterisierungen verliefen analog zu der oben beschriebenen Homopolymerisation.

\subsubsection{Abspaltung des an Silica gebundenen Polymers}

Das mit lebenden (Blockco-)Polymerketten bedeckte Silica wurde in einer mit Argon befüllten Handschuhbox mit einer Lösung von $0.5 \mathrm{~mol} \cdot \mathrm{L}^{-1}$ AIBN in Toluol, die vorher mittels dreimaligem „freeze-pump-and-thaw“ entgast wurde, gelöst. Die Sus- 
pension wurde bei $80^{\circ} \mathrm{C}$ für drei Stunden intensiv gerührt. Nach der Polymerisation wurden die Silicapartikel filtriert, und das Filtrat wurde mittels GPC analysiert.

\subsection{Ergebnisse und Diskussion}

\subsubsection{Die chemische Verankerung über Trimethoxysilan}

Das RAFT-Agens 2 wird an der Oberfläche des Silica mit Hilfe der Trimethoxysilangruppe verankert. Das Silan besitzt im Allgemeinen einen organischen Substituent $\mathrm{R}$ und drei hydroxylierbare Substituenten. Abbildung 4.3 zeigt schematisch den Mechanismus der chemischen Verankerung an einer hydroxylhaltigen Oberfläche mit Hilfe der Trimethoxysilangruppe (im linken Kasten).

Die Verankerung verläuft über vier Schritte. Beim ersten Schritt werden die Methoxysilangruppen durch Hydrolyse mit Wasser in Silanol umgewandelt. Die silanolhaltige Spezies kondensieren und formen im zweiten Reaktionsschritt Oligomere ${ }^{2}$. Anschließend bilden die Oligomere mit den Hydroxyl-Gruppen auf der Oberfläche des Substrates Wasserstoffbrückenbindungen, die relativ stark sind. Schließlich wird eine kovalente Bindung mit der Oberfläche des Substrates unter Abspaltung eines Wassermoleküls gebildet.

Es soll hier angemerkt werden, dass die Trimethoxysilangruppe nicht nur an die Oberfläche von Silica gebunden werden kann. Sie kann im Prinzip an alle Oberflächen, die O-H-Gruppen enthalten, nach dem oben dargestellten Mechanismus fest fixiert werden. Abbildung 4.3 zeigt im rechten Kasten eine Auswahl von den möglichen anorganischen Substraten, an denen die Trimethoxysilangruppe mit unterschiedlicher Effektivität verankert werden kann [88, 89]. Silica ist für diesen Zweck ein exzellentes Substrat. Außerdem ist Silica chemisch resistent, mechanisch stabil und kostengünstig. Daher wird in dieser Arbeit Silica als Modellsubstrat für die heterogene RAFT-Polymerisation verwendet.

\footnotetext{
${ }^{2}$ Die Hydrolyse und die Kondensation des Polymers mit Trimethoxysilangruppe, welches dann in z. B. Stern- oder Kammpolymer umgewandelt wird, werden in Kapitel 7 detailliert diskutiert.
} 


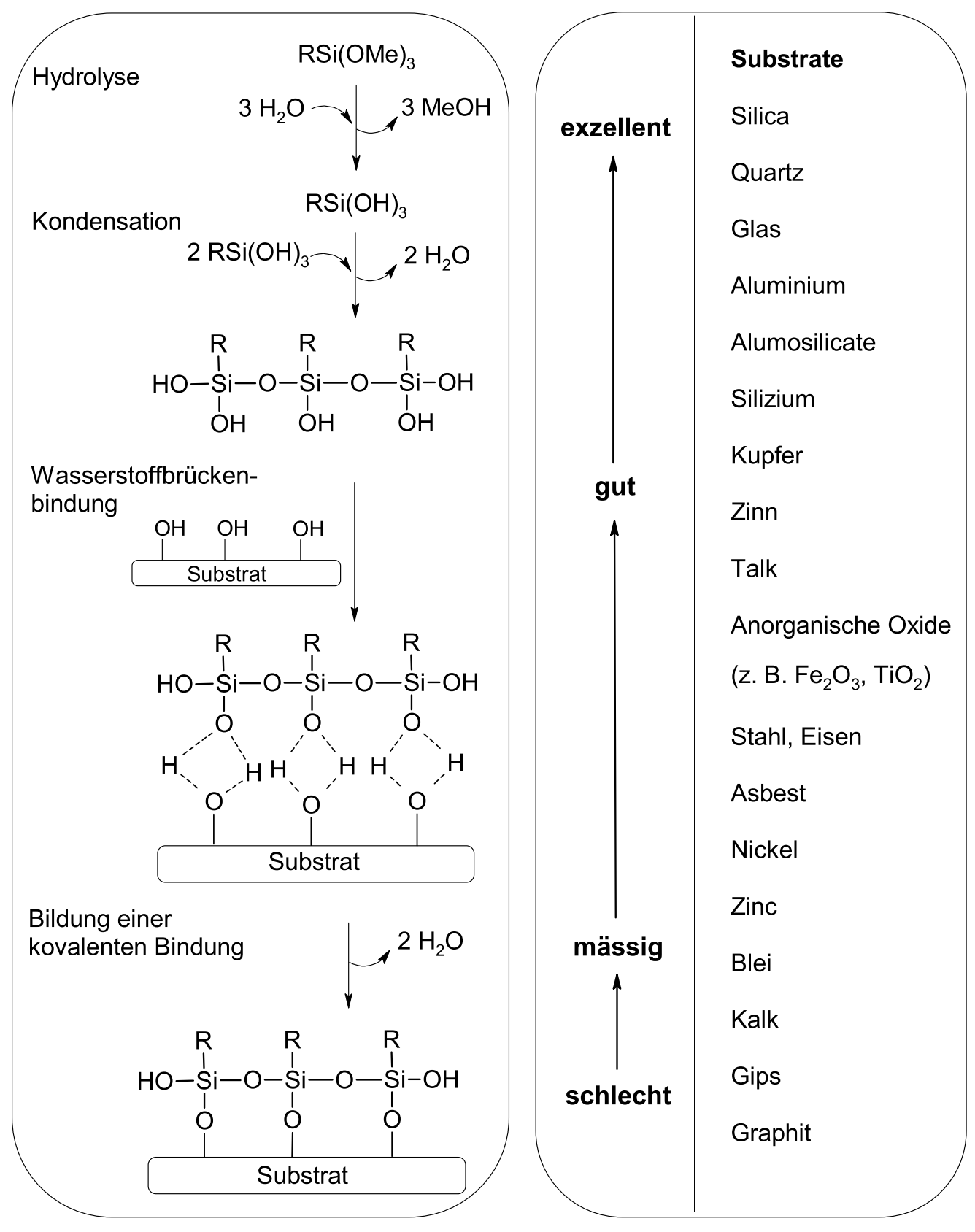

Abbildung 4.3: Mechanismus der Verankerung von Trimethoxysilangruppe an hydroxylhaltigen Oberflächen und die Effektivität bei geeigneten anorganischen Substraten [87].

\subsubsection{Homogene RAFT-Polymerisation}

Eine erfolgreiche heterogene RAFT-Polymerisation setzt eine effektive homogene RAFT-Polymerisation mit dem entsprechenden ungebundenen RAFT-Agens vor- 
aus. In diesem Abschnitt soll die Eigenschaften des verwendeten Trithiocarbonats als freies ungebundenes RAFT-Agens kurz dargestellt werden. Eine detaillierte Beschreibung befindet sich in Kapitel 7.

Das ungebundene freie RAFT-Agens 4 (Propyl(phenylmethyl)trithiocarbonat, PPT), das die gleiche funktionelle Trithiocarbonatgruppe wie das gebundene RAFTAgens 3 besitzt, ist für die kontrollierte Polymerisation von Styrol gut geeignet. Aber wegen der relativ schlechten Abgangsgruppe von PPT zeigt die Polymerisation von Styrol ein leichtes Hybridverhalten in der Anfangsphase ${ }^{3}$. Das Hybridverhalten verursacht eine Verbreiterung der Molmassenverteilung des gebildeten Polystyrols, dessen Polydispersität ca. 1.3 beträgt.

Für die Polymerisation von Methylacrylat hingegen ist das PPT ein sehr gutes RAFT-Agens. Das aus der RAFT-Polymerisation erhaltene Polymethylacrylat hat eine Polydispersität kleiner als 1.1, d. h. auf eine extrem enge Molmassenverteilung. Außerdem scheint die Verwendung von Toluol für die kontrollierte Lösungspolymerisation von Methylacrylat problemlos möglich.

Die Herstellung des Blockcopolymer aus Styrol und Methylacrylat ist relativ einfach durchzuführen. Dabei ist es gleichgültig, ob das Styrol oder das Methylacrylat zuerst polymerisiert wird. Aus diesen Gründen werden sowohl Styrol als auch Methylacrylat als Monomer für die heterogene RAFT-Polymerisation mit dem am Silica gebundenen PPT ausgewählt.

\subsubsection{Heterogene RAFT-Polymerisation}

Als Lösungsmittel für die heterogene RAFT-Polymerisation wird Toluol verwendet, um ein starkes Ansteigen der Viskosität der Polymerisationslösung bei höherem Monomerumsatz zu verhindern. Eine zu hohe Viskosität behindert das Durchmischen der Polymerisationssuspension, die sehr intensiv gerührt werden muss, damit das propagierende Radikal die Trithiocarbonatgruppe an der Oberfläche erreichen kann. Für die heterogene RAFT-Polymerisation von Methylacrylat ist ein großer Massenanteil des Lösungsmittel von bis zu 90 \% erforderlich, um eine gut kontrollierte Polymerisation zu erreichen.

\footnotetext{
${ }^{3}$ Das Hybridverhalten zeichnet sich durch einen sprunghaften Anstieg der Molmasse in der Anfangsphase, gefolgt von einer kontrollierten Polymerisation.
} 


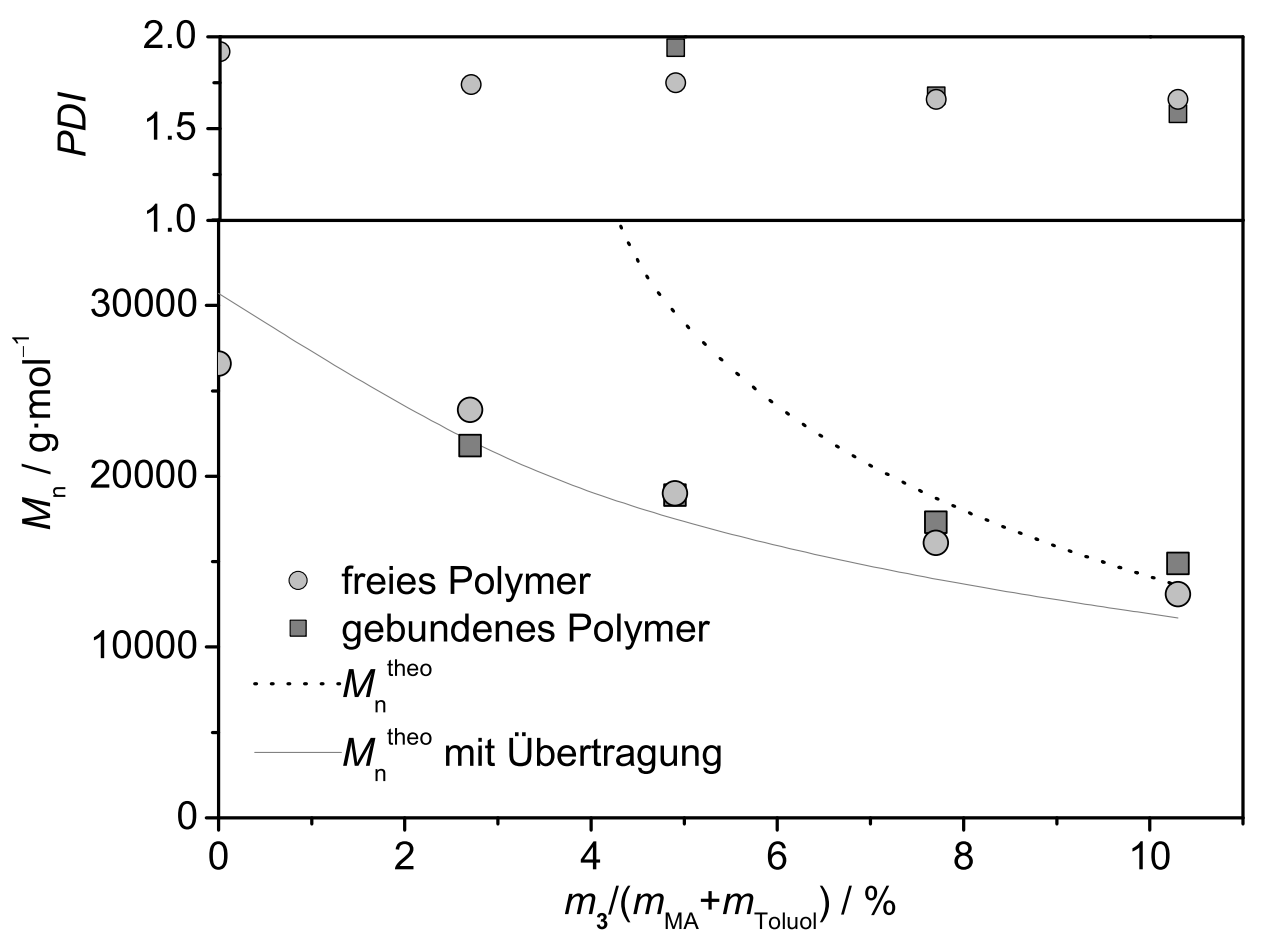

Abbildung 4.4: Auftragung der mittleren Molmasse $M_{\mathrm{n}}$ und Polydispersität PDI gegen das Massenverhältnis von modifiziertem Silica 3 zu der gesamten Reaktionslösung für die Polymerisation von Methylacrylat bei $60{ }^{\circ} \mathrm{C}$, mit $\operatorname{AIBN}\left(1.2 \cdot 10^{-3} \mathrm{~mol} \cdot \mathrm{L}^{-1}\right)$ als Initiator. Der Umsatz betrug bei einer Reaktionszeit von 5.0 Stunden ca. 58 \% für allen Proben. Die Polymerisation mit der höchsten Konzentration von 3 wurde mit einem Molverhältnis von $n$ (Monomer) $: n$ (gebundenes RAFT-Agens) $: n(\mathrm{AIBN})=1000: 3.9: 1.04$ durchgeführt.

Abbildung 4.4 zeigt die Ergebnisse der heterogenen RAFT-Polymerisation von Methylacrylat unter Variation des Massenanteils vom eingesetzten modifizierten Silica 3. Dabei sind sowohl die Werte des freien Polymethylacrylat als auch die des gebundenen Polymers, das mit einer überschüssigen Menge des Initiators AIBN von der Silica-Oberfläche abgespalten wird, aufgetragen. Für die Polymerisationsreihe, bei der kein freies RAFT-Agens hinzu gegeben wird, ist das am Silica gebundene Trithiocarbonat das einzige kontrollierende Agens. Da das Trithiocarbonat durch die stabilisierende Z-Gruppe chemisch an der Silica-Oberfläche verankert ist, bildet das gebundene Polymer die lebende polymere Fraktion, während das freie Polymer 
die tote Fraktion darstellt.

Bei gleichem Monomerumsatz ist die mittlere Molmasse des Polymers indirekt proportional zu der Konzentration des RAFT-Agens, weil die Anzahl der Polymerkette, bei Vernachlässigung des geringen toten Polymersmaterials, der Anzahl der RAFT-Agens-Moleküle entspricht. Wie erwartet, fällt die mittlere Molmasse mit der steigenden Menge des modifizierten Silica ab. Die Kettenlängen des freien und gebundenen Polymers sind fast identisch, was mit der Simulation der RAFTPolymerisation (siehe Abbildung 3.6 in Kapitel 3) gut zusammen passt.

Das theoretische Zahlenmittel der Molmasse $M_{\mathrm{n}}^{\text {theo }}$ für gesamtes Polymer wird wie folgt berechnet:

$$
M_{\mathrm{n}}^{\text {theo }}=\frac{m_{\mathrm{M}} \cdot U_{\mathrm{M}}}{n_{\text {gebunden }}+n_{\text {frei }}+2 \cdot n_{\mathrm{I}}}
$$

In dieser Gleichung sind $m_{\mathrm{M}}$ die Masse des Monomers, $U_{\mathrm{M}}$ der fraktionelle Monomerumsatz, $n_{\text {gebunden }}$ die Stoffmenge von gebundenem Trithiocarbonat, $n_{\text {frei }}$ die Stoffmenge von freiem Trithiocarbonat und $n_{\mathrm{I}}$ • die Stoffmenge von effektivem primärem Radikal aus dem Initiatorszerfall. Die Terminierung verläuft bei Methylacrylat über Kombination, wobei aus zwei propagierenden Radikalen eine Polymerkette entsteht.

Die theoretische mittlere Molmasse ist viel höher als die experimentell ermittelten Werte. Besonders stark ist diese Abweichung bei niedrigem Massenanteil des modifizierten Silica. Für das Experiment ohne RAFT-Agens hat das erhaltene Polymethylacrylat eine mittlere Molmasse von $26.6 \mathrm{~kg} / \mathrm{mol}$, während die theoretische Molmasse bei $387.0 \mathrm{~kg} / \mathrm{mol}$ liegt. Der große Unterschied der beiden Werte weist auf einen systematischen Fehler bei der Berechnung der theoretischen Molmasse hin.

Eine beim Vergleich mit $M_{\mathrm{n}}^{\text {theo }} \mathrm{zu}$ niedrige $M_{\mathrm{n}}$ hat seine Ursache oft bei der unberücksichtigen Transferreaktion. Ein möglicher Transferreaktionspartner ist neben dem Monomer selbst das Lösungsmittel Toluol, da Toluol bis zu 90 \% der gesamten Masse der Polymerisationslösung stellt. Die Übertragungskonstante $C_{\text {tr }}$ von Methylacrylat zum Toluol beträgt $2.7 \cdot 10^{-4}$ bei $60^{\circ} \mathrm{C}[90]$. Obwohl der Geschwindigkeitskoeffizient für die Transferreaktion des propagierenden Methylacrylat-Radikals zum Toluol sehr klein ist, hat dieser Reaktionspfad doch einen signifikanten Einfluss auf das polymere Produkt. Besonders für die Fälle, bei denen die Anzahl der Polymerendgruppen von Initiator und von RAFT-Agens niedrig ist, ist der Einfluss der Transferreaktion von Toluol groß und darf nicht mehr vernachlässigt wer- 
den. Gleichung 4.2 stellt die korrigierte Berechung der theoretischen Molmasse unter Berücksichtigung der Transferreaktion dar.

$$
M_{\mathrm{n}}^{\text {theo }}=\frac{m_{\mathrm{M}} \cdot U_{\mathrm{M}}}{n_{\text {gebunden }}+n_{\text {frei }}+2 \cdot n_{\mathrm{I}} \cdot+q \cdot t}
$$

Dabei steht $q \cdot t$ für die Anzahl der zusätzlichen Polymerendgruppen, die durch Transferreaktionen entstanden sind. Sie ist bei gleich bleibender Toluol- und Radikalkonzentration in der stationären Phase eine lineare Funktion der Zeit. $q$ ist eine für diese Polymerisationsreihe angepasste Konstante ${ }^{4}$.

Wie man in Abbildung 4.4 erkennen kann, gibt der korrigierte Verlauf der theoretischen Molmasse den experimentellen Werte viel besser wieder. Bei hohem Massenanteil des modifizierten Silica von $10 \%$ spielt die Transferreaktion kaum noch eine Rolle und kann deshalb zur Vereinfachung vernachlässigt werden.

Die Polydispersität des freien und gebundenen Polymers ist größer als 1.5 und nimmt mit steigendem Massenanteil des modifizierten Silica ab, weil die Konzentration des kontrollierenden Agens und damit auch die Kontrolle der Polymerisation entsprechend zunehmen. Die breite Molmassenverteilung wird begründet durch die relativ schlechte Erreichbarkeit der Trithiocarbonatgruppe an der Silica-Oberfläche. Die Erreichbarkeit der Trithiocarbonatgruppe für die Radikale ist relativ schlecht im Falle einer Verankerung des RAFT-Agens über die stabilisierende Z-Gruppe, die von der langen Polymerkette sterisch abgeschirmt wird. Dieser sterische Abschirmungseffekt nimmt mit steigender Kettenlänge des Polymers stark zu.

Die mittlere Molmasse des Polymers hängt direkt von der Konzentration des kontrollierenden Agens ab, wie man aus Gleichung 4.1 sehen kann. Ein großer Unterschied der lokalen Konzentration in der Polymerisationsmischung, wie es bei einer heterogenen Reaktion inhärent ist, führt unweigerlich zur Verbreiterung der Molmassenverteilung des entstehenden Polymers. Durch Hinzufügen eines freien ungebundenen RAFT-Agens kann dieser Unterschied in den lokalen RAFT-AgensKonzentrationen ausgeglichen werden, und eine gut kontrollierte RAFT-Polymerisation wird somit möglich.

\footnotetext{
${ }^{4}$ Eine quantitative Abschätzung für $q$ ist wegen der unbekannten und schwierig zu bestimmenden Radikalkonzentration nicht möglich.
} 


\subsubsection{Kombination von homogener und heterogener RAFT- Polymerisation}

Um die Kontrolle der heterogenen RAFT-Polymerisation zu erhöhen und damit eine engere Molmassenverteilung des polymeren Produktes zu erhalten, wird zusätzlich zu dem gebundenen Trithiocarbonat 3 ein freies, ungebundenes RAFT-Agens 4 in die Polymerisationsmischung gegeben. Die Ergebnisse dieser Polymerisationsreihe sind in Abbildung 4.5 abgebildet.

Wie erwartet, haben sowohl das freie als auch das gebundene Polymethylacrylat eine enge Molmassenverteilung, was aus dem PDI kleiner als 1.5 ersichtlich ist. Das freie RAFT-Agens hat eine gute Kontrolle der heterogenen RAFT-Polymerisation von Methylacrylat ermöglicht.

Durch eine höhere RAFT-Agens-Konzentration erhöht sich entsprechend die Anzahl der Polymerkette. Dadurch ist die mittlere Molmasse beim Vergleich mit der Polymerisationsreihe, bei der die heterogene Polymerisation ohne freies RAFTAgens durchgeführt wird, deutlich kleiner. Die sterische Abschirmung wird wegen der kürzeren Polymerketten verringert, was die Erreichbarkeit der Trithiocarbonatgruppen an der Oberfläche erhöht. Der Unterschied der lokalen RAFT-AgensKonzentration wird durch das freie RAFT-Agens reduziert, was einheitliche Kettenlängen bewirkt.

Bei der heterogenen kontrollierten radikalischen Polymerisation findet ein gewisser Anteil der Reaktion in der flüssigen Phase unkontrolliert statt. Das freie RAFT-Agens hingegen unterdrückt durch seine Anwesenheit die unkontrollierte Polymerisation in der flüssigen Phase, wodurch die mittlere Molmasse des freien und gebundenen Polymers im Rahmen der experimentellen Unsicherheiten annähernd übereinstimmen.

Die theoretische Molmasse $M_{\mathrm{n}}^{\text {theo }}$, welche für das gesamte Polymerisationssystem berechnet wurde, stimmt mit dem experimentellen Werte gut überein. Nur bei den Experimenten mit geringer RAFT-Agens-Konzentration treten kleine Abweichungen auf. Diese Abweichungen können durch die oben beschriebene einmalige Transferreaktion erklärt werden.

Die systematische Untersuchung der Entwicklung der mittleren Molmasse mit dem Monomerumsatz ist erforderlich, um wichtige Kenntnisse über die Effizient einer 


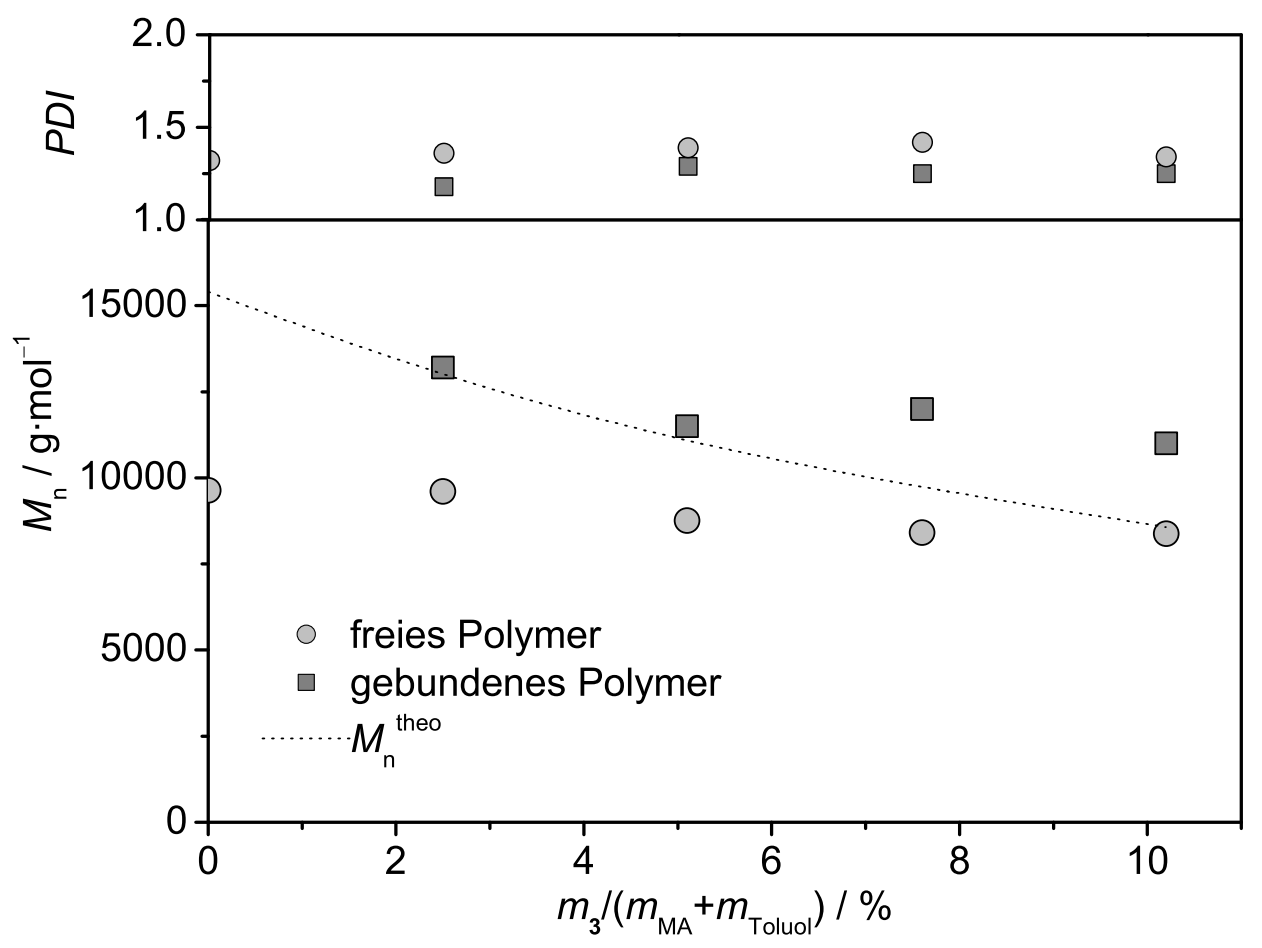

Abbildung 4.5: Auftragung der mittleren Molmasse $M_{\mathrm{n}}$ und Polydispersität PDI gegen das Massenverhältnis von modifiziertem Silica $3 \mathrm{zu}$ der gesamten Reaktionslösung für die Polymerisation von Methylacrylat bei $60{ }^{\circ} \mathrm{C}$ mit zusätzlichem freiem RAFT-Agens $\left([4]=4.8 \cdot 10^{-3} \mathrm{~mol} \cdot \mathrm{L}^{-1}\right)$, initiiert durch AIBN $\left(1.2 \cdot 10^{-3} \mathrm{~mol} \cdot \mathrm{L}^{-1}\right)$. Der Umsatz beträgt bei einer Reaktionszeit von 15 Stunden ca. 79 \% für allen Proben. Die Polymerisation mit der höchsten Konzentration von 3 wird mit einem Molverhältnis von $n$ (Monomer) : $n$ (gebundenes RAFT-Agens) $: n($ freies RAFT-Agens) $: n($ AIBN $)=1000: 3.9: 4.1: 1.04$ durchgeführt.

kontrollierten Polymerisation zu erlangen. Dabei kann man ein umfassendes Bild der Polymerisation von der Anfangsphase bis zum vollständigen Monomerumsatz machen. Abbildung 4.6 zeigt die Entwicklung der Molmasse und der Polydispersität in Abhängigkeit vom Monomerumsatz für die heterogene RAFT-Polymerisation von Methylacrylat mit dem am Silica gebundenem Trithiocarbonat 3.

Wie man in Abbildung 4.6 erkennen kann, nimmt die mittlere Molmasse des freien und gebundenen Polymers linear mit dem Monomerumsatz zu. Die Polydi- 


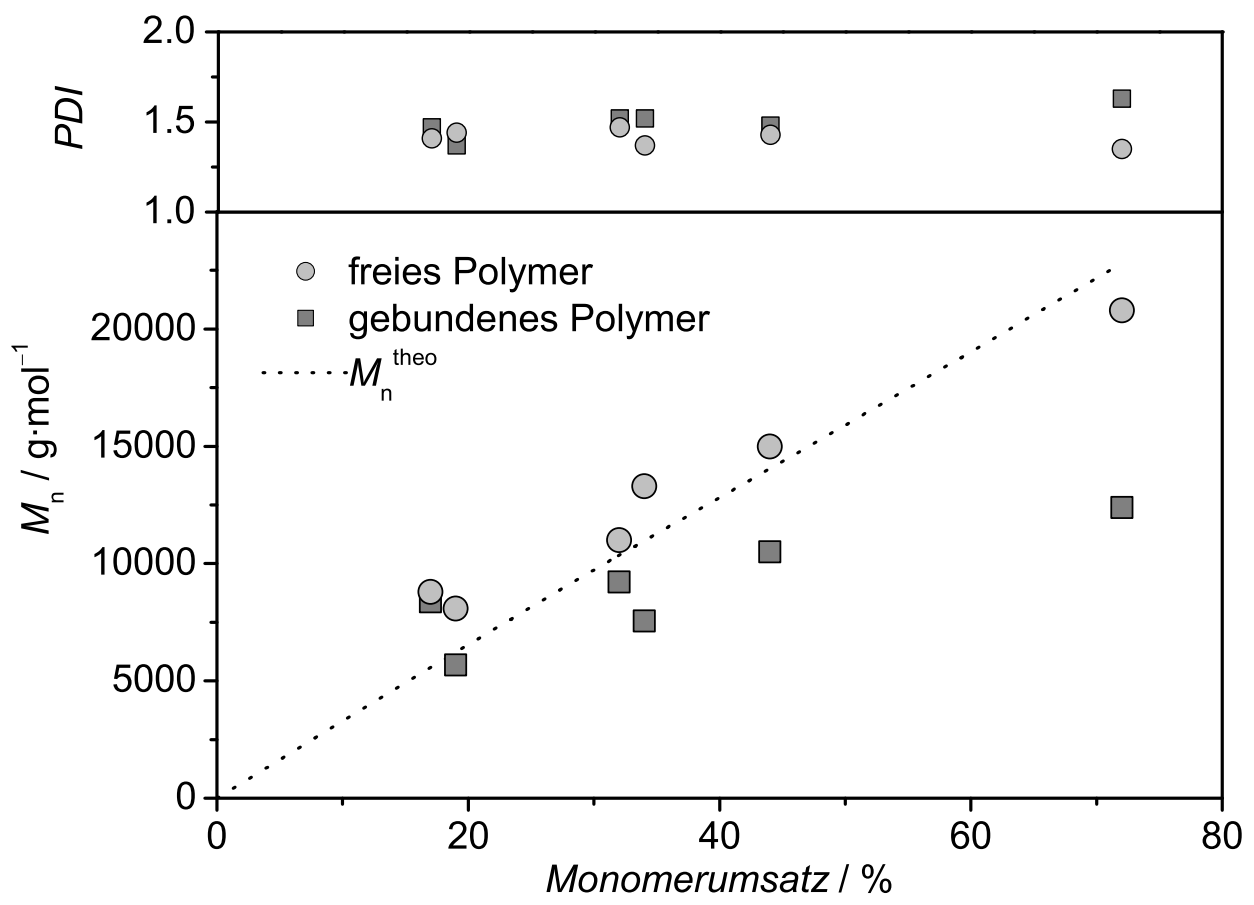

Abbildung 4.6: Auftragung der mittleren Molmasse $M_{\mathrm{n}}$ und Polydispersität PDI gegen den Monomerumsatz für die Polymerisation von Methylacrylat bei $60{ }^{\circ} \mathrm{C}$ mit einem Massenanteil des modifizierten Silica von $10 \%$ und $1.0 \cdot 10^{-3} \mathrm{~mol} \cdot \mathrm{L}^{-1}$ AIBN. Die Polymerisation wurde mit einem Stoffmengenverhältnis von $n$ (Monomer) : $n$ (gebundenes RAFT-Agens) : $n($ freies RAFT-Agens) $: n($ AIBN $)=1000: 1.4: 2.0: 0.28$ in Toluol (Massenanteil $70 \%)$ durchgeführt.

spersität ist für eine kontrollierte Polymerisation relativ hoch, aber immer noch kleiner als 1.5. Diese beiden Beobachtungen weisen auf eine effektive heterogene RAFT-Polymerisation hin.

Bei dieser Polymerisationsreihe wurde ein höherer Massenanteil des Monomers von $30 \%$ eingesetzt, um die Transferreaktion zum Lösungsmittel zu verringern. Die erhöhte Monomer-Konzentration könnte der Hauptgrund dafür sein, dass die Polydispersität in dieser Reihe signifikant größer ist als bei Experimenten mit einem Massenanteil vom Methylacrylat von $10 \%$.

Bei der genauen Betrachtung der mittleren Molmasse fällt auf, dass die Ket- 
tenlängen des freien und gebundenen Polymers nur bis zu einer Molmasse von 10 $\mathrm{kg} / \mathrm{mol}$ übereinstimmen. Oberhalb von $10 \mathrm{~kg} / \mathrm{mol}$ ist das freie Polymer deutlich größer als das gebundene. Das bedeutet, dass ab einer Molmasse von $10 \mathrm{~kg} / \mathrm{mol}$ das direkt an der Silica-Oberfläche gebundene Trithiocarbonat für das propagierende Radikal nicht mehr problemlos zu erreichen ist. An diesem kritischen Punkt verlangsamt sich das Wachstum der lebenden Polymerketten an der Oberfläche, während die RAFT-Polymerisation in der Lösung weiter abläuft.

\subsubsection{Charakterisierung}

Die direkte Charakterisierung der gebundenen Polymere ist wegen der eingeschränkten Möglichkeiten der spektroskopischen Methoden schwierig. Die in Lösung routinemäßig durchgeführte NMR-Spektroskopie liefert aufgrund starker Signalverbreitungen durch die gehinderte Beweglichkeit der immobilisierten Substrate kaum verwertbare Ergebnisse [91]. Neuere Entwicklungen wie die MAS-Techniken („magic angle spinning“) in der Festkörper-NMR-Spektroskopie versprechen Abhilfe. Wegen der fehlenden Flüchtigkeit lassen sich gebundene Verbindungen auch nicht mit herkömmlichen Methoden massenspektroskopisch untersuchen. Sehr schnell und einfach lässt sich die IR-Spektroskopie durchführen.

Um das gebundene Polymethylacrylat direkt zu charakterisieren, ohne es von der Silica-Oberfläche abzuspalten, wurden hier die ATR-FTIR-Spektroskopie und die Festkörper-NMR-Spektroskopie eingesetzt.

\section{ATR-FTIR-Spektroskopie}

Die ATR-FTIR-Spektroskopie ${ }^{5}$ eignet sich hervorragend für die Charakterisierung von Materialoberflächen. Mit dieser ATR-Technik lassen sich Proben untersuchen, die für Transmissions-Messungen unbrauchbar sind, wie z. B. Pasten, Kunststofffasern oder Beschichtungen von Oberflächen.

Die verwendete ATR-Einheit hat einen Diamantkristall als Träger für die Substanzen. Da der Diamantkristall für die IR-Strahlung in Wellenlängenbereich von 1900 bis $2300 \mathrm{~cm}^{-1}$ nicht durchlässig ist, enthält das ATR-FTIR-Spektrum in diesem Bereich keine Information. Dies ist kaum ein Nachteil des Diamantkristalls, weil

\footnotetext{
${ }^{5}$ Attenuated Total Reflection = abgeschwächte Totalreflexion
} 


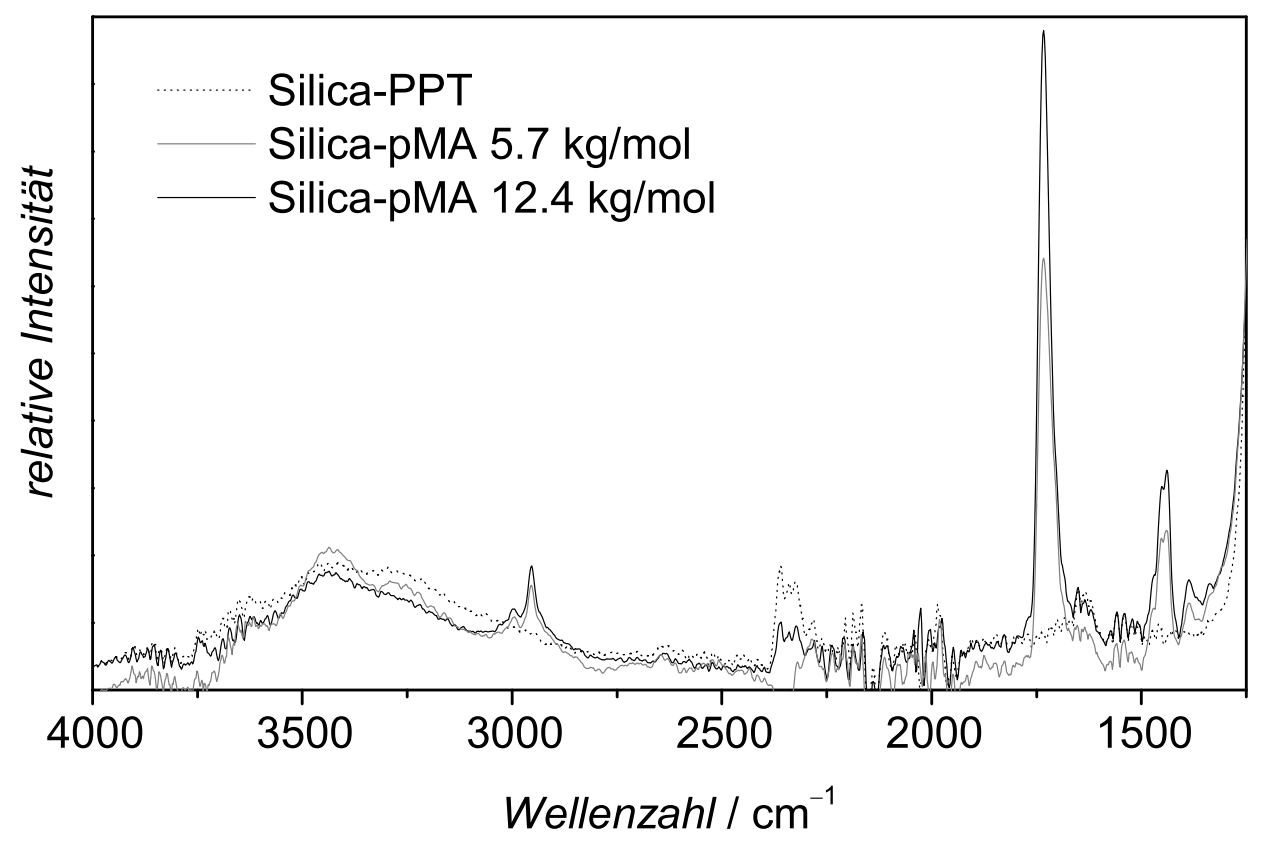

Abbildung 4.7: FTIR-Spektren, die mit der ATR-Technik aufgenommen wurden, von dem modifizierten Silica vor und nach der heterogenen RAFT-Polymerisation von Methylacrylat. Die Spektren wurden über das O-H-Schwingungssignal bei $3400 \mathrm{~cm}^{-1}$ normiert.

organische Substanzen kaum Schwingungssignale in diesem IR-Bereich besitzen.

In Abbildung 4.7 sind die FTIR-Spektren, die mit der ATR-Technik aufgenommen wurden, von den modifizierten Silica vor und nach der heterogenen RAFTPolymerisation gezeigt. Die modifizierten Silica-Proben enthalten nach der Polymerisation gebundenes Polymethylacrylat mit unterschiedlichen mittleren Molmassen.

Das unbehandelte Silica hat im gemessenen IR-Bereich nur ein breites Signal der O-H-Schwingung der Silanolgruppe bei $3400 \mathrm{~cm}^{-1}$. Das modifizierte Silica, das eine Beladungsdichte der Trithiocarbonatgruppe von $0.048 \mathrm{mmol} / \mathrm{g}$ hat, unterscheidet sich in seinem FTIR-Spektrum kaum von dem des unbehandelten Silica. Warum die Signale des gebundenen RAFT-Agens nicht sichtbar sind, liegt wohl daran, dass die Schwingungsbanden des RAFT-Agens wegen seiner geringen Menge zu schwach sind und vom Rauschen überlagert werden.

Das Spektrum des modifizierten Silica, das nach der RAFT-Polymerisation das gebundene Polymethylacrylat mit der mittleren Molmasse von $5.7 \mathrm{~kg} / \mathrm{mol}$ enthält, 
zeigt im Vergleich zum Spektrum des unbehandelten Silica ein hohes Signal bei $1730 \mathrm{~cm}^{-1}$. Da Carbonylgruppen eine charakteristische starke Absorption in dem Wellenzahlbereich um $1730 \mathrm{~cm}^{-1}$ aufweisen, darf man annehmen, dass das modifizierte Silica die Acrylat-Estergruppen enthält. Auch das relativ breite Signal bei $2950 \mathrm{~cm}^{-1}$ kommt von den C-H-Schwingungen des gebundenen Polymethylacrylats.

Beim quantitativen Vergleich der beiden FTIR-Spektren des modifizierten Silica mit unterschiedlichen $M_{\mathrm{n}}$ kann man erkennen, dass das Signal bei $1730 \mathrm{~cm}^{-1}$ bei der Probe mit höherem $M_{\mathrm{n}}$ deutlich größer ist als das bei kleinerem $M_{\mathrm{n}}$. Eine quantitative Analyse des gebundenen Polymers, mit der die $M_{\mathrm{n}}$-Werte direkt gemessen werden können, setzen gleich bleibende Bedingungen des Messexperiments und eine sorgfältige Kalibrierungen voraus. Die weitere Entwicklung und Optimierung dieser quantitativen Analysemethode würde den Rahmen dieser Arbeit sprengen und wird deshalb hier nicht weiter verfolgt.

\section{Festkörper-NMR-Spektroskopie}

Die Festkörper-NMR-Spektroskopie ${ }^{6}$ ist eine sehr geeignete Methode zur Strukturaufklärung, weil sie die Möglichkeit bietet, Aussagen über die Nahordnung in der Umgebung der betrachteten Kernspinsorte zu treffen [92, 93]. Abbildung 4.8 zeigt das ${ }^{13} \mathrm{C}-\mathrm{NMR}$-Spektrum von dem modifizierten Silica $\mathbf{3}$ mit dem gebundenen Trithiocarbonat, das mittels Festkörper-NMR-Technik aufgenommen wurde.

Das ${ }^{13} \mathrm{C}$-Festkörper-NMR-Spektrum von 3 gibt die NMR-Signale vom gebundenen Trithiocarbonat beim Vergleich mit den Werten aus der Lösung-NMR-Spektroskopie gut wieder (vergleiche mit Abbildung 7.3 in Kapitel 7). Das starke Signal bei der chemischen Verschiebung von 130 ppm kann klar den Kohlenstoffatomen des Phenylringes zugeordnet werden. Die Signale 1 bis 4 gehören ebenso eindeutig den Kohlenstoffatomen des gebundenen RAFT-Agens.

Interessant ist das Signal 5, das zu dem Kohlenstoffatom der Methoxygruppe gehört. Die Fläche des Signals 5 ist etwas kleiner als die Signale 1 bis 4 . Das bedeutet, dass jedes gebundene Trithiocarbonat-Molekül im Schnitt etwas weniger als eine Methoxygruppe von den drei Ursprünglichen besitzt. Die zwei fehlenden Methoxygruppe sind bereits für die chemische Verankerung abreagiert. Dies beweist eine feste chemische Bindung zwischen dem Trithiocarbonat und der Silica-Oberfläche mit ca.

\footnotetext{
${ }^{6}$ Kernmagnetische Resonanzspektroskopie (nuclear magnetic resonance $=$ NMR).
} 


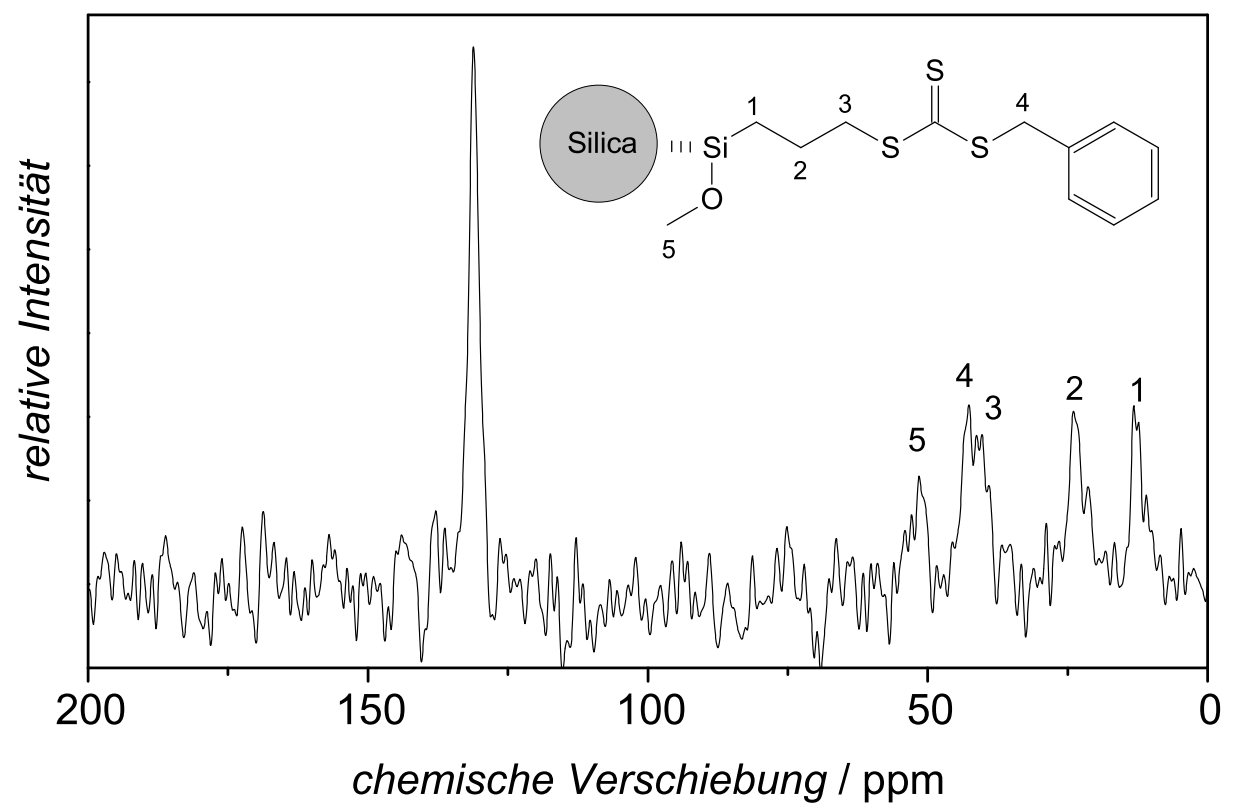

Abbildung 4.8: Das ${ }^{13}$ C-Festkörper-NMR-Spektrum vom modifizierten Silica vor der Polymerisation, das an der Oberfläche das RAFT-Agens enthält.

zwei Silanolgruppen.

Das Kohlenstoffatom der Thiocarbonylgruppe ist in dem ${ }^{13} \mathrm{C}$-Festkörper-NMRSpektrum nicht sichtbar. Dieses Kohlenstoffatom liefert erfahrungsgemäß ein sehr schwaches NMR-Signal, das normalerweise bei sehr hoher chemischer Verschiebung von ca. $230 \mathrm{ppm}$ zu finden ist. Wegen der generell schlechten Signalintensität der Festkörper-NMR-Technik ist es nicht verwunderlich, dass das Signal der Thiocarbonylgruppe vom Rauschen überlagert wird.

Das in Abbildung 4.8 dargestellte ${ }^{13} \mathrm{C}$-Festkörper-NMR-Spektrum hat ein relativ schlechtes Signal-Rausch-Verhältnis, obwohl 76800 Scans für das Spektrum aufgenommen wurden, was eine mehrtägige Messzeit am Spektrometer erforderte. Mehr Scans wären wünschenswert, war aber aufgrund der beschränkten Messzeit nicht möglich.

Auch das ${ }^{13}$ C-Festkörper-NMR-Spektrum in Abbildung 4.9 wurde mit 76800 Scans aufgenommen. Es ist vom modifizierten Silica, das nach der RAFT-Polymerisation das gebundene Polymethylacrylat mit einer mittleren Molmasse von 


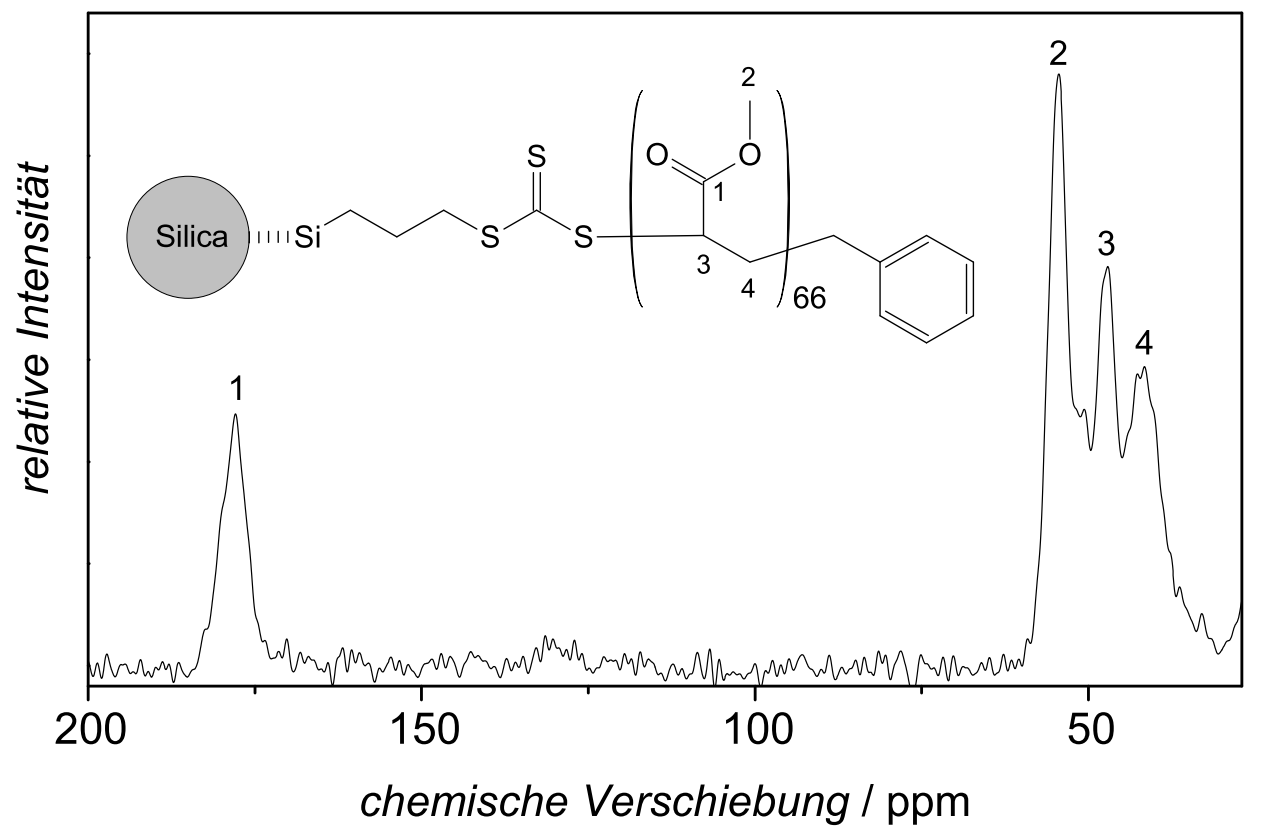

Abbildung 4.9: ${ }^{13}$ C-Festkörper-NMR-Spektrum vom modifizierten Silica nach der Polymerisation von Methylacrylat, das an der Oberfläche die Polymerkette enthält.

$5.7 \mathrm{~kg} / \mathrm{mol}$ trägt. Durch die Erhöhung des Kohlenstoffsanteiles in dem Hybridmaterial ist das Signal-Rausch-Verhältnis deutlich gestiegen.

Die Zuordnung der Signale ist mit Hilfe der Werte aus der Lösung-NMR-Spektroskopie für das Polymethylacrylat problemlos und eindeutig möglich. Die unterschiedliche Intensität der Signale ist auf die Abschirmung des jeweiligen Kohlenstoffatoms zurück zu führen. Deshalb zeigt die Methoxygruppe das intensivste Signal, weil sie am wenigsten abgeschirmt ist.

Die erwarteten chemischen Strukturen des gebundenen Trithiocarbonat und des immobilisierten Polymethylacrylats können durch die Festkörper-NMR-Spektroskopie eindeutig bestätigt werden.

\subsubsection{Glasübergangstemperatur}

Materialeigenschaften wie Zugfestigkeit, Maßstabilität und Elastizität hängen bei Polymeren ganz entscheidend von den intermolekularen Wechselwirkungen der Poly- 
merketten untereinander ab. Diese Wechselwirkungen zeigen charakteristische Temperaturabhängigkeiten, und ihre Änderung ist in der Regel mit einem Wärmeumsatz verbunden.

Die DSC (Differential Scanning Calorimetry) ist eine kalorimetrische Methode zur Untersuchung der intermolekularen Wechselwirkung zwischen den Polymerketten. In der DSC wird der Wärmefluss zu einer Polymerprobe als Funktion der Temperatur gemessen, während die Temperatur der Probe in definierter Weise geändert wird. DSC-Messungen erlauben die Ermittlung von Enthalpien chemischer Reaktionen und physikalischer Umwandlungen wie von Schmelz- und Glasübergangstemperaturen.

Die Glasübergangstemperatur (Glastemperatur) ist einer der wichtigsten Parameter, die den möglichen Anwendungsbereich eines nicht kristallinen polymeren Materials bestimmt. Bei dieser Temperatur gehen ziemlich harte, glasartige Massen in weichere gummiartige Materialien über. Die Abhängigkeit der Glastemperatur $T_{\mathrm{g}}$ von der Molmasse lässt sich durch die sogenannte Fox-Flory-Gleichung beschrieben [94].

$$
T_{\mathrm{g}}=T_{\mathrm{g} \infty}-\frac{K}{M}
$$

wobei $T_{\mathrm{g} \infty}$ die Glastemperatur des Polymers mit unendlich großer Molmasse, $M$ das Molekulargewicht des Polymers und $K$ eine Konstante ist. Die Fox-FloryGleichung besagt, dass die Glastemperatur sich umgekehrt proportional zur Molmasse des Polymers verhält.

Eine weitere Möglichkeit, die Glastemperatur zu verändern, ist die Verwendung von Nanopartikeln, die wegen ihres großen Oberfläche-Volumen-Verhältnisses einen sehr großen Einfluss auf die Beweglichkeit der Polymerkette haben. In der Literatur wurde die Wirkung der Nanopartikeln auf die Glastemperatur sehr intensiv beschrieben, weil man damit den Temperaturbereich für mögliche Anwendungen vergrößern kann [95, 96, 97]. Durch Einsatz von Nanopartikeln kann die Glastemperatur eines polymeren Materials sowohl erhöht [98, 99] als auch gesenkt [100, 101] werden.

Für den Fall, dass das Polymer und die Nanopartikel inkompatibel sind, gibt es keine Wechselwirkung zwischen den Polymerketten und der Partikeloberfläche. Die für das Polymer glatte Oberfläche erhöht die Beweglichkeit der Polymerketten, wodurch die Glastemperatur gesenkt wird [102]. Umgekehrt kann die Glastemperatur 

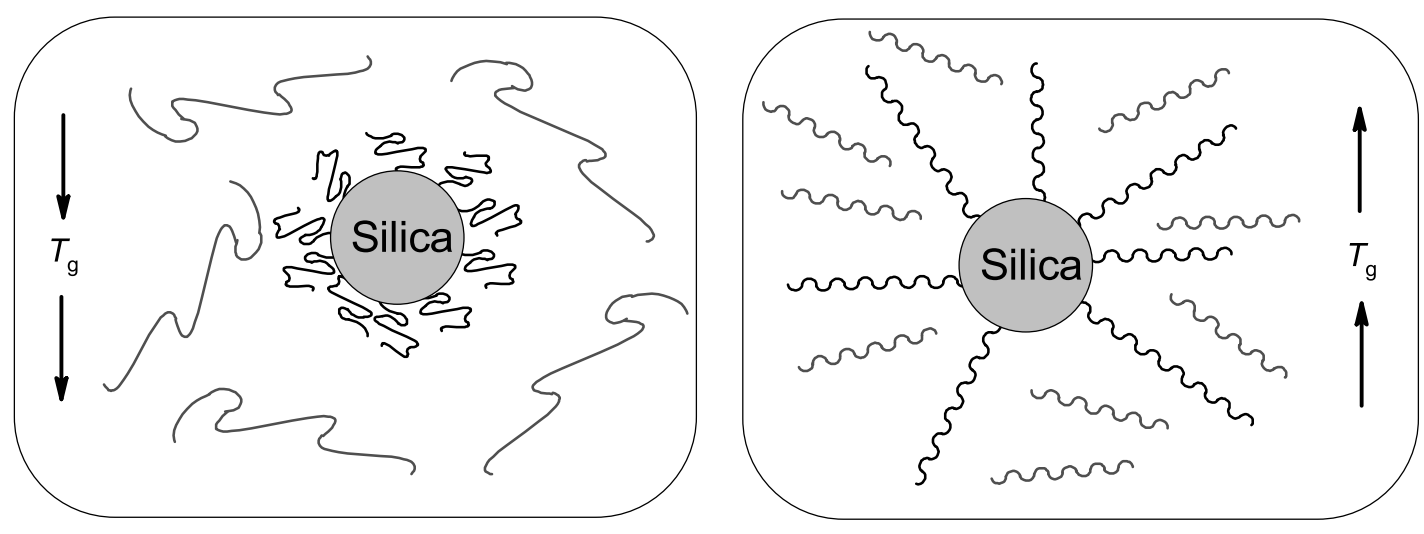

Abbildung 4.10: Schematische Darstellung des Einflusses der Nanopartikeln bzw. ihrer Oberfläche auf die Glastemperatur.

mit steigendem Nanopartikelgehalt steigen, wenn es eine starke Wechselwirkung zwischen beiden Phasen gibt. Denn die Beweglichkeit der Polymerkette wird dadurch stark gebremst [103, 104].

Bei einer Mischung bestehend aus freiem Polymer und an Nanopartikeln gebundenem Polymer gleicher Art können folgende Situationen auftreten:

1. Das freie Polymer hat eine viel größere Kettenlänge als das gebundene, wodurch die langen freien Polymerketten nicht die Möglichkeit haben mit den Nanopartikel in Wechselwirkung zu treten. Folglich sinkt die Glastemperatur mit steigendem Partikelgehalt, weil die freien Polymerketten eine höhere Beweglichkeit an der glatten Partikel-Oberfläche aufweisen (siehe Abbildung 4.10, linkes Schema).

2. Die freien Polymerketten sind kürzer als die gebundenen und können mit der Oberfläche des Nanopartikels in Wechselwirkung treten. Folglich sinkt die Beweglichkeit des freien Polymers, was eine Erhöhung der Glastemperatur bewirkt. Dabei stehen die gebundenen Polymerketten tendenziell senkrecht auf der Oberfläche der Nanopartikel (siehe Abbildung 4.10, rechtes Schema).

Abbildung 4.11 zeigt die experimentell ermittelten Glastemperaturen der polymeren Produkte, die aus heterogener RAFT-Polymerisation von Styrol mit unter- 


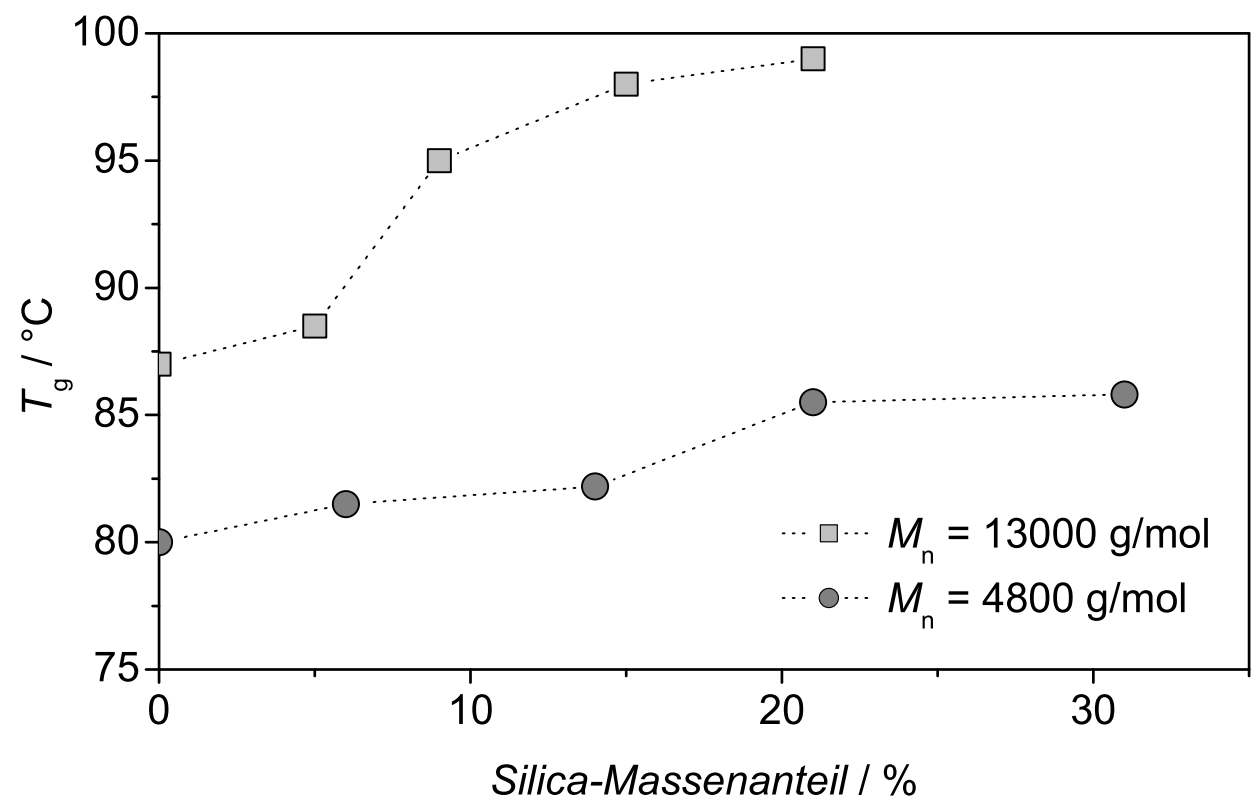

Abbildung 4.11: Glastemperaturen der polymeren Produkte, die aus heterogener RAFTPolymerisation von Styrol mit unterschiedlichen Konzentrationen des gebundenen und freien Trithiocarbonats entstanden sind. Die Glastemperatur wurde aus DSC-Experimenten bestimmt.

schiedlichen Konzentrationen des gebundenen und freien Trithiocarbonats entstanden sind.

Wie in Abbildung 4.5 und 4.6 bereits erwähnt, haben das freie und gebundene Polymer etwa die gleiche Kettenlänge. Das Polystyrol aus den beiden Polymerisationsreihen hat eine mittlere Molmasse von $4800 \mathrm{~g} / \mathrm{mol}$ bzw. $13000 \mathrm{~g} / \mathrm{mol}$. Das kürzere Polymer hat entsprechend der Fox-Flory-Gleichung eine niedrigere Glastemperatur als das Polymer mit dem höheren Molekulargewicht.

Bei beiden Polymerisationsreihen steigt die Glastemperatur mit dem zunehmenden Silicagehalt. Mit den hergestellten Nanopartikeln, deren Oberfläche mit Polystyrol modifiziert wurde, kann man die Glastemperatur von Polystyrol um bis zu $10{ }^{\circ} \mathrm{C}$ erhöhen. Somit wurde eine Methode zur Variation der Glastemperatur entwickelt, was aus industrieller Sicht interessant sein könnte.

Die Erhöhung der Glastemperatur mit steigendem Nanopartikelgehalt bedeutet, 
dass das freie Polymer starke Wechselwirkungen mit der Silica-Oberfläche eingeht, was die Beweglichkeit der Polymerkette behindert. Das bedeutet auch, dass die gebundenen Polymerketten tendenziell senkrecht auf der Oberfläche stehen, wodurch die Trithiocarbonatgruppen gut zugänglich sind. Auch wenn die DSC-Messungen die Glastemperaturen von Feststoffmischungen bestimmen, kann man aus den Ergebnissen Rückschlüsse auf die Vorgänge während der heterogenen RAFT-Polymerisation schließen.

Für eine erfolgreiche RAFT-Polymerisation an der Oberfläche muss die Trithiocarbonatgruppe, welche über die stabilisierende Z-Gruppe fest verankert ist, für das propagierende Radikal erreichbar sein. Dafür ist es vorteilhaft, wenn das gebundene Polymer tendenziell senkrecht zur Oberfläche steht, und das propagierende Makroradikal mit Silica-Oberfläche wechselwirken kann. Beide Annahmen werden durch die DSC-Experimente zur Bestimmung der Glastemperatur bestätigt.

\subsubsection{Synthese des gebundenen Blockcopolymers}

Die durch RAFT-Polymerisationen erhaltenen Polymere stellen makromolekulare Kettenüberträger dar, die zur Synthese von wohl definierten Blockcopolymere benutzt werden können. Um das kontrollierte Verhalten einer heterogenen RAFTPolymerisation mit dem am Silica gebundenen Trithiocarbonat zu demonstrieren, werden gebundene Blockcopolymere aus Polymethylacrylat mit Styrol synthetisiert. Die Daten des Homopolymers und Blockcopolymer sind in Tabelle 4.1 zusammengefasst. Die zugehörigen GPC-Diagramme sind in Abbildung 4.12 dargestellt. Die GPC-Eichung des Blockcopolymers wurde mit Hilfe der Mark-Houwing-Koeffizienten von Polymethylacrylat durchgeführt ${ }^{7}$.

Das kontrollierte Verhalten der RAFT-Polymerisation zur Herstellung des zweiten Blocks zeigt sich in der Entwicklung der Molmassenverteilung in Abbildung 4.12. Die Molmassenverteilungen des gebundenen und freien Polymers verschieben sich nach der RAFT-Polymerisation von Styrol zu größeren Molekulargewichten.

\footnotetext{
${ }^{7}$ Bei der Bestimmung der Molmassenverteilung von Copolymeren dagegen werden meist nur scheinbare Werte ermittelt, denn eine Bestimmung der Molmasse in Abhängigkeit vom Eluationsvolumen ist nicht eindeutig, da das hydrodynamische Volumen nicht unbedingt linear mit der Copolymerzusammensetzung zusammenhängt.
} 


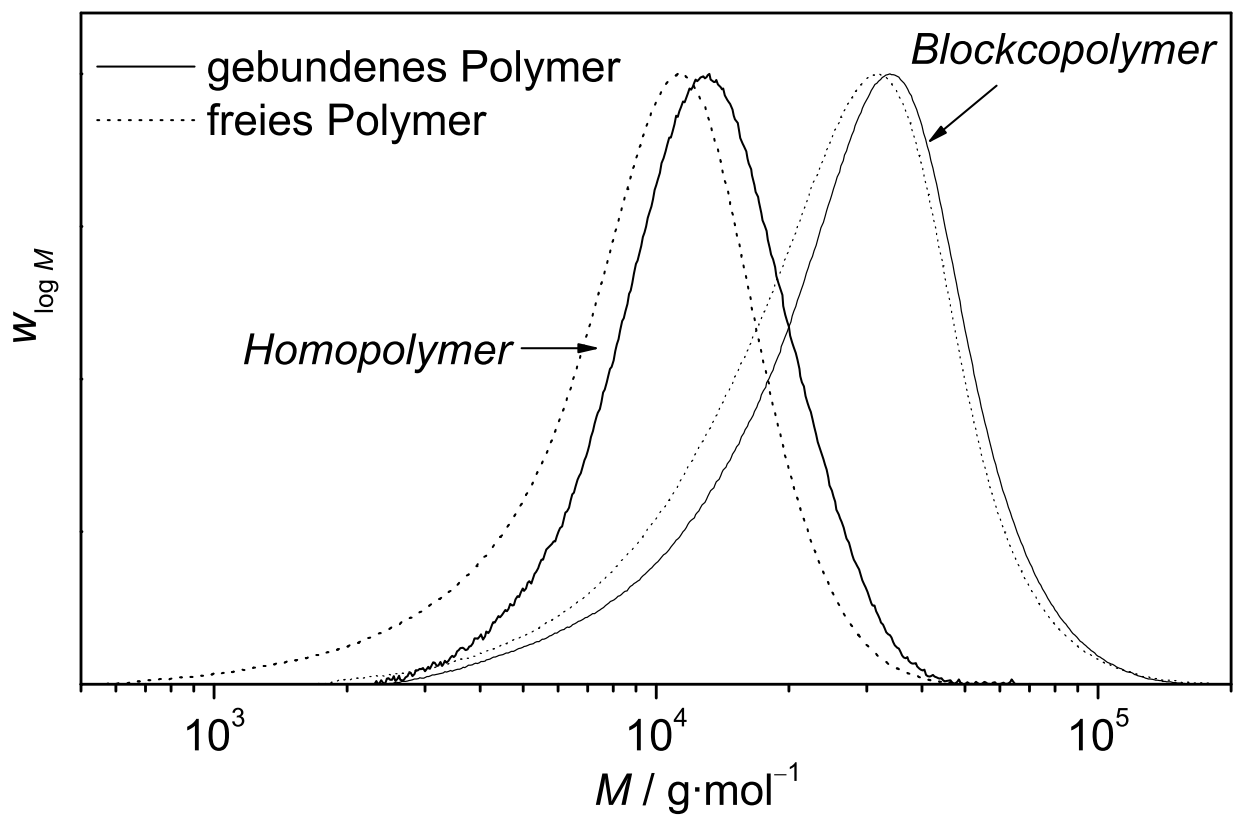

Abbildung 4.12: Molmassenverteilung des Polymethylacrylat und des Blockcopolymers PMA-b-PSty, das bei der heterogenen RAFT-Polymerisation von Methylacrylat und Styrol bei $60{ }^{\circ} \mathrm{C}$ mit AIBN (ca. $1.0 \cdot 10^{-3} \mathrm{~mol} \cdot \mathrm{L}^{-1}$ ) entstanden ist. Die gepunktete Linie zeigt das freie Polymer, das in der flüssigen Phase gebildet wurde. Die durchgezogene Linie gehört dem gebundenen Polymer, das nach der Polymerisation vom Silica abgespalten wurde.

\begin{tabular}{l|llllll}
\hline & $\begin{array}{l}\text { Monomer- } \\
\text { umsatz }\end{array}$ & $\begin{array}{l}M_{\mathrm{n}}^{\text {theo }} \\
\mathrm{g} / \mathrm{mol}\end{array}$ & $\begin{array}{l}M_{\mathrm{n}}^{\text {frei }} \mathrm{g} / \mathrm{mol} \\
P D I^{\text {frei }}\end{array}$ & $\begin{array}{l}M_{\mathrm{n}}^{\text {gebunden }} \\
\mathrm{g} / \mathrm{mol}\end{array}$ & $P D I^{\text {gebunden }}$ \\
\hline $\begin{array}{l}\text { Homopolymer } \\
\text { pMA }\end{array}$ & & 8570 & 10900 & 1.25 & 8380 & 1.34 \\
\hline Blockcopolymer & $23 \%$ & 14700 & 15400 & 1.33 & 13900 & 1.42 \\
pMA-b-pSt & $41 \%$ & 19400 & 21400 & 1.42 & 18700 & 1.53 \\
\hline
\end{tabular}

Tabelle 4.1: Mittlere Molmassen $M_{\mathrm{n}}$ und Polydispersität $P D I$ des freien und gebundenen Blockcopolymers, das aus der heterogenen RAFT-Polymerisation von Methylacrylat und Styrol mit dem gebundenen Trithiocarbonat entstanden ist.

Das erfolgreich synthetisierte, an Silica-Nanopartikel gebundene Blockcopolymer besitzt ein Molekulargewicht $M_{\mathrm{n}}^{\text {gebunden }}$ von $13900 \mathrm{~g} / \mathrm{mol}(P D I=1.42)$ bei einem Styrolumsatz von 23 \%, bzw. $18700 \mathrm{~g} / \mathrm{mol}(P D I=1.53)$ bei einem Styrolumsatz 
von $41 \%$. Die mittlere Molmasse steigt mit dem Styrolumsatz an, eine Tatsache, die in Einklang mit dem für eine kontrollierte radikalische Polymerisation geforderten Zusammenhang steht. Gleichzeitig besitzen die Blockcopolymere eine niedrigen PDI (kleiner als 1.5). Tabelle 4.1 zeigt außerdem die gute Übereinstimmung zwischen experimentell erhaltenen und theoretisch berechneten Molmassen. Die experimentell ermittelten Daten unterstützen damit das erwartete Verhalten von gebundenem Trithiocarbonat bei der Polymerisation von Styrol zur Herstellung des zweiten Blocks. Dies alles zeigt, dass die Polymerisation mit dem gebundenen Trithiocarbonat unter den beschriebenen Bedingungen kontrolliert abläuft.

\subsection{Schlußfolgerung}

Die Immobilisierung eines Trithiocarbonats, das eine Trimethoxysilangruppe zur chemischen Verankerung an Silica-Oberflächen besitzt, wurde detailliert untersucht.

Die radikalische Polymerisation von Styrol bzw. Methylacrylat wurde bei der Verwendung des gebundenen Trithiocarbonats bezüglich seiner Molmasse erfolgreich kontrolliert. Die Methode der Immobilisierung des RAFT-Agens über die Z-Gruppe erlaubt die Herstellung von schwefelfreiem Polymermaterial mit kontrollierter Molmasse und bietet einen Weg zur Wiedergewinnung des gebundenen RAFT-Agens. Zusätzlich ermöglicht die Fixierung des RAFT-Agens an Silicapartikeln eine effektive Trennung des lebenden Polymers von dem toten Polymer, das während der RAFT-Polymerisation gebildet wird.

Zur effektiven Oberflächenmodifizierung kann der Polymerisationsmischung zusätzlich zu dem gebundenen Trithiocarbonat ein freies RAFT-Agens zugesetzt werden, um eine engere und definiertere Molmassenverteilung des polymeren Produktes zu erhalten. Es wurde gezeigt, dass die Synthese von wohldefinierten, an der Oberfläche gebundenen Blockcopolymeren mit der Methode der Immobilisierung des RAFT-Agens über die Z-Gruppe möglich ist.

Für eine direkte Charakterisierung des modifizierten Silica wurden die ATRFTIR-Spektroskopie und die Festkörper-NMR-Spektroskopie erfolgreich eingesetzt. Beide Methode bestätigen die erwartete Strukturen des an Silica-Oberfläche gebundenen RAFT-Agens sowie des gebundenen Polymers. 
Die modifizierten Silica-Nanopartikel, die durch die heterogene RAFT-Polymerisation Polymerketten an der Oberfläche tragen, sind in ersten Versuchen auf deren Eigenschaften als Füllstoffe untersucht worden. Es hat sich dabei gezeigt, dass man durch Verwendung der modifizierten Nanopartikel die für die Bearbeitung und Anwendung wichtige Glastemperatur des polymeren Materials variieren kann. Eine steigende Glastemperatur des polymeren Produktes mit steigendem Silica-Gehalt bestätigt die Annahme, dass das gebundene Polymer tendenziell senkrecht zur Oberfläche steht und dass das propagierende Makroradikal mit der Silica-Oberfläche wechselwirken kann. 


\section{Kapitel 5}

\section{An Wang-Harz gebundenes Xanthat}

In den beiden vorherigen Kapiteln wurden die heterogenen RAFT-Polymerisationen mit den an Silica-Nanopartikel gebundenen Dithio- und Trithioverbindungen vorgestellt. In diesem Kapitel werden die Immobilisierung von Xanthaten und die zugehörige heterogene RAFT-Polymerisation von Vinylacetat beschrieben. Bei der kontrollierten radikalischen Polymerisation von Vinylacetat werden Xanthate als RAFT-Agenzien benötigt, da durch Dithibenzoate und Trithiocarbonate eine unzureichende Kontrolle erfolgt. Anstatt des anorganischen Silica wird ein polymerer Träger, nämlich Wang-Harz, als festes Substrat verwendet.

\section{$5.1 \quad$ Einführung}

Die Weiterentwicklung der kontrollierten radikalischen Polymerisation erfolgt mit rasanter Geschwindigkeit, weil sie die Kontrolle der Architektur von polymeren Materialien ermöglicht. Gegenwärtig sind NMP ( $N$ itroxide $M$ ediated Polymerization [15, 17]), ATRP (Atom Transfer Radical Polymerization [18, 4]), RAFT-Polymerisation (Reversible Addition Fragmentation Chain Transfer [19, 20]) und MADIX (Macromolecular Design via the Interchange of $X$ anthates $[105,106])$ die bekanntesten Methoden. RAFT und MADIX sind bezüglich ihres Mechanismus identisch und stellen zurzeit wohl die vielfältigste kontrollierte Polymerisationsmethode in Bezug auf verwendbare Monomere und Reaktionsbedingungen dar. Bis heute ist die kontrollierte 


\section{Vorgleichgewicht}

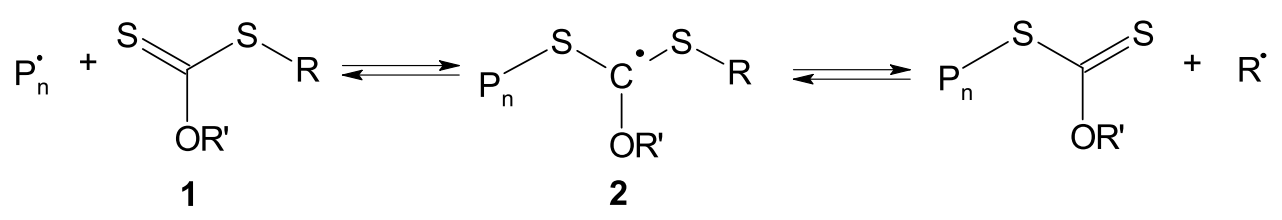

Hauptgleichgewicht

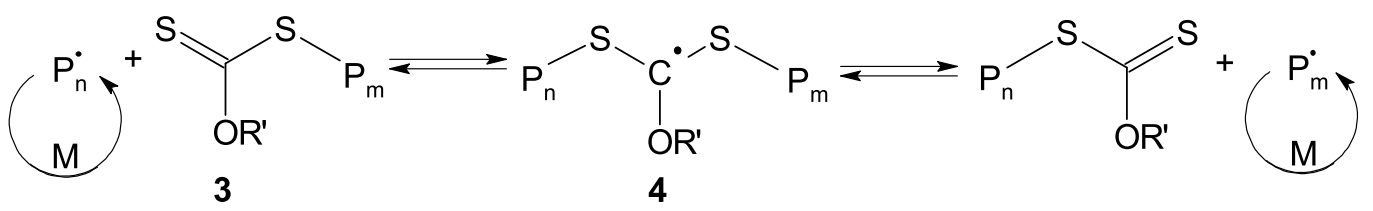

Abbildung 5.1: Vor- und Hauptgleichgewichte in der MADIX-Polymerisation.

radikalische Polymerisation von Vinylacetat nur mit der effektiven MADIX-Methode möglich.

Die hohe Reaktivität des propagierenden Radikals bei der Vinylacetat-Polymerisation gestaltet die Reaktionskontrolle im Vergleich zu anderen Monomeren schwierig. Alkyljodid [107], Dithiocarbamat [108] und Xanthat [45, 109] werden laut Literatur als geeignete RAFT-Agenzien für die Polymerisation von Vinylacetat eingesetzt. Aber nur die MADIX-Polymerisation, bei welcher ein Xanthat als RAFT-Agens verwendet wird, ist zur Herstellung von Polyvinylacetat mit einer kleinen Polydispersität und einer definierten Architektur geeignet. Bei der Verwendung eines Xanthats als RAFT-Agens wird die Elektronendichte am zentralen Kohlenstoffatom erhöht, wodurch die Stabilität der intermediären RAFT-Radikale 2 und 4 verringert wird. Dadurch wird die Instabilität des propagierenden Vinylester-Radikals ausgeglichen. Außerdem wird die Additionsgeschwindigkeit des propagierenden Radikals zu 1 und 3 wegen der geringen Resonanzstabilisierung, welche die Doppelbindungscharakter der C-S-Bindung von 1 und $\mathbf{3}$ einschränkt, verringert.

Systeme von den an Festkörper gebundenen Katalysatoren für kontrollierte Polymerisation wurden z. B. für NMP $[47,48,49]$, ATRP $[50,51,52]$ und RAFT [71, 110, 111] beschrieben. Mit diesen Methoden können eine ganze Reihe von Polymerketten 
mit einer definierten Architektur an unterschiedlichen Trägern wie Silica, Silizium, Gold, Baumwolle, Zellulose, polymeren Trägern, etc. gebunden werden. Aber es gibt bis heute noch keine Berichte über ein System von heterogenem Katalysator für die kontrollierte Polymerisation von Vinylacetat. Diese Arbeit beschreibt die MADIXPolymerisation von Vinylacetat mit Hilfe von Xanthaten, die chemisch über die stabilisierende Z-Gruppe an polymeren Trägern gebunden sind.

Merrifield berichtete als erster über chemische Reaktionen von Substraten, die an polymeren Trägern gebunden waren [112]. Er entwickelte eine automatisierbare Methode zur effizienten Synthese von Peptiden und wurde dafür 1984 mit dem Nobelpreis ausgezeichnet. Dieses revolutionäre Verfahren mit polymeren Trägern wird heute in vollautomatischen Syntheseautomaten zum Aufbau von Peptiden und Oligonucleotiden verwendet $[113,114]$.

Die Verwendung von polymeren Trägern scheint sehr gut im Hinblick auf mögliche Anwendungen in der bio-organischen Chemie geeignet zu sein. Eine detaillierte Erforschung der RAFT-Polymerisation an polymeren Trägern erscheint interessant und vielversprechend.

\subsection{Experiment}

\subsubsection{Synthese von an Wang-Harz gebundenem Xanthat}

Die neu entwickelte Synthese von an Wang-Harz gebundenem Xanthat basiert auf der von Perrier [115] entwickelten Methode zur Herstellung von Xanthaten (siehe Abbildung 5.2). Es ist eine „one-pot“-Synthese, die in Abbildung 5.3 schematisch illustriert wird. Bei der Reaktion wird 1,1'-Thiocarbonyldiimidazol 5 mit einem Alkohol und einem Thiol umverestert.

Um ein freies Xanthat nach dieser Methode zu synthetisieren, muss zuerst die Umesterung von 1,1'-Thiocarbonyldiimidazol mit einem Alkohol durchgeführt werden. Danach führt die zweite Umesterung mit einem Thiol zu dem gewünschten Xanthat. Bei der Umkehrung dieser Reihenfolge entsteht kein Xanthat, sondern ein Thiocarbonat $^{1}$ als Hauptprodukt, da das entstehende Xanthat eine reaktive Zwischenstufe darstellt und entsprechend weiter reagiert.

\footnotetext{
${ }^{1}$ Der systematische Name für die Xanthatgruppe ist Dithiocarbonat.
} 
5<smiles>S=C(n1ccnc1)n1ccnc1</smiles><smiles>CCOC(=O)C(C)S</smiles><smiles></smiles><smiles>CCOC(=O)C(C)SC(=S)I</smiles><smiles>CCOCC(C)C</smiles><smiles>CCOC(=S)n1ccnc1</smiles><smiles>CCOC(=O)C(C)SC(=S)OCC</smiles>

Abbildung 5.2: Syntheseroute zu freien Xanthat 7 für die MADIX-Polymerisation [115].

Das Wang-Harz enthält an seiner Oberfläche fest gebundene Alkoholgruppen. Bei einer Beladungsdichte von $1.1 \mathrm{mmol} / \mathrm{g}$ sind ungefähr $10 \%$ der Phenylringe des Polystyrol-Grundgerüstes funktionalisiert. Dies bedeutet, dass die einzelnen gebundenen Alkoholgruppen so weit voneinander getrennt sind, als ob sie sich in einer hoch verdünnten Lösung befänden. Dies ermöglicht die einseitige Umesterung der reaktiven Zwischenstufe zur Bildung von gebundenem Xanthat, die unter vergleichbaren Bedingungen in Lösung nicht realisierbar wäre. Eine weitere Umesterung von Xanthat zu Thiocarbonat kann nicht stattfinden, weil sowohl die Alkoholgruppe als auch das entstehende Xanthat an der Oberfläche fest fixiert und sich weit voneinander befinden. Für die Synthese des gebundenen Xanthates kann daher die Reihenfolge der Umesterungsreaktionen umgedreht werden, um die am Wang-Harz haftenden Nebenprodukte möglichst gering zu halten.

\section{Synthese des freien Xanthates 7}

1,1'-Thiocarbonyldiimidazol (5, TCDI, $1.78 \mathrm{~g}, 10.0 \mathrm{mmol}$ ) wurde in trockenem Toluol (60 mL) unter Stickstoff gelöst. Zu der entstehenden Lösung wurde Ethanol (0.46 g, $10.0 \mathrm{mmol})$ tropfenweise hinzugegeben. Die Mischung wurde bei $60{ }^{\circ} \mathrm{C}$ für 


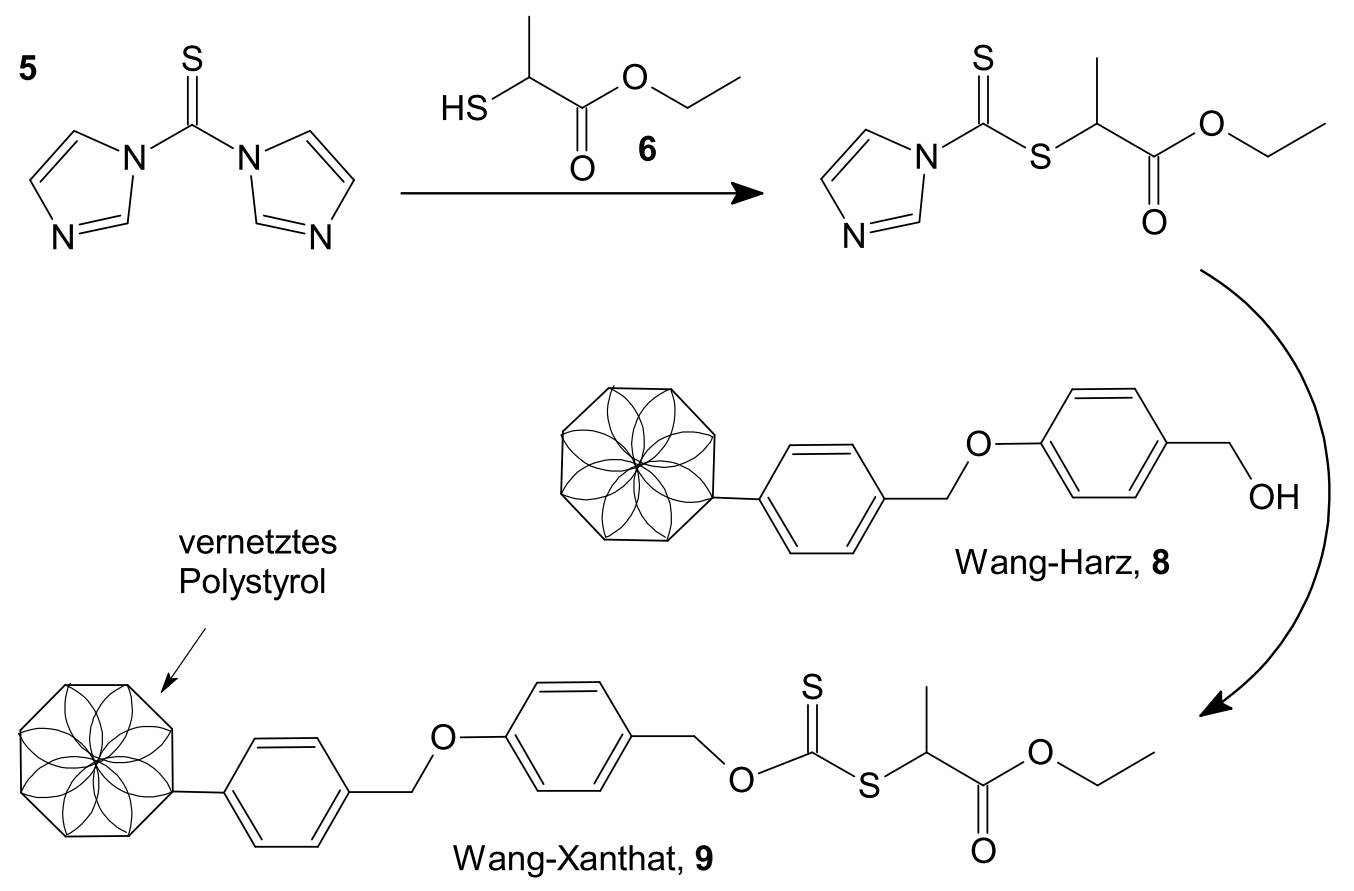

Abbildung 5.3: Syntheseroute zu einem Xanthat, das über die stabilisierende Z-Gruppe an der Oberfläche von Wang-Harz chemisch gebunden ist.

6 Stunden erhitzt und bei der Zimmertemperatur über Nacht gerührt. Anschließend wurde 2-Mercaptopropansäureethylester (6, 1.34 g, 10.0 mmol) zu der Reaktionslösung gegeben. Die Suspension wurde wieder bei $60{ }^{\circ} \mathrm{C}$ für 6 Stunden erhitzt. Nachdem das Lösungsmittel unter vermindertem Druck entfernt wurde, wurde das Produkt säulenchromatographisch gereinigt (Silicagel, Pentan : Ethylacetat $=9: 1$, $\left.R_{\mathrm{f}}=0.57\right)$. Man erhält das freie Xanthat 7 als schwach gelbliche Flüssigkeit (1.3 g, $5.8 \mathrm{mmol}, 58 \%)$.

${ }^{1} \mathrm{H}-\mathrm{NMR}\left(300 \mathrm{MHz}, \mathrm{CDCl}_{3}\right), \delta(\mathrm{ppm}): 1.3\left(\mathrm{t}, 3 \mathrm{H},-\mathrm{CH}_{3}\right), 1.4\left(\mathrm{t}, 3 \mathrm{H},-\mathrm{CH}_{3}\right), 1.6$ $\left(\mathrm{d}, 3 \mathrm{H},-\mathrm{CH}_{3}\right), 4.2\left(\mathrm{q}, 2 \mathrm{H},-\mathrm{O}-\mathrm{CH}_{2}-\mathrm{CH}_{3}\right), 4.8$ (q, 1H, $\left.-\mathrm{S}-\mathrm{CH}_{1}-\mathrm{CH}_{3}\right), 5.7$ (q, 2H, $\left.-\mathrm{O}-\mathrm{CH}_{2}-\mathrm{CH}_{3}\right)$.

${ }^{13} \mathrm{C}-\mathrm{NMR}(300 \mathrm{MHz}, \mathrm{CDCl} 3), \delta(\mathrm{ppm}): 14.4$ und $17.2\left(\mathrm{CH}_{2}-\mathrm{CH}_{3}\right), 31.1\left(\mathrm{CH}_{1}-\right.$ $\left.C \mathrm{H}_{3}\right), 48.8$ und $51.8\left(\mathrm{CH}_{2}-\mathrm{CH}_{3}\right), 62.1\left(\mathrm{CH}_{1}-\mathrm{CH}_{3}\right), 172.3(C(=\mathrm{O}) \mathrm{O}), 213.7(C=\mathrm{S})$.

Synthese des gebundenen Xanthates $\mathbf{9}$

Unter Stickstoff wurde 1,1'-Thiocarbonyldiimidazol (5, $3.60 \mathrm{~g}, 19.2 \mathrm{mmol})$ in tro- 
ckenem Toluol (60 mL) unter Stickstoff-Gegenstrom gelöst. Zu der Lösung wurde 2-Mercaptopropansäureethylester (6, $2.71 \mathrm{~g}, 19.2 \mathrm{mmol})$ tropfenweise hinzu gegeben. Die Mischung wurde bei $60{ }^{\circ} \mathrm{C}$ für 6 Stunden erhitzt und bei Zimmertemperatur über Nacht gerührt.

Das Wang-Harz (8, 5.0 g, $5.3 \mathrm{mmol}$ OH-Gruppe) wurde zu der stark rührenden Reaktionslösung unter Stickstoff-Gegenstrom hinzugegeben. Die Suspension wurde erneut bei $60{ }^{\circ} \mathrm{C}$ für 6 Stunden gerührt. Nachdem die Reaktionsmischung auf Zimmertemperatur abgekühlt war, wurde sie gefiltert. Das erhaltene und modifizierte Wang-Harz 9 wurde gründlich mit Toluol, Aceton, THF und Dichlormethan gewaschen und unter Vakuum bei Zimmertemperatur über Nacht getrocknet.

Aus der Elementaranalyse wurde ein Schwefelmassenanteil von $1.3 \%$ bestimmt. Daraus konnte die Beladungsdichte von Wang-Harz mit Xanthat berechnet werden. Sie betrug $0.20 \mathrm{mmol} / \mathrm{g}$ modifiziertes Wang-Harz, was eine Ausbeute von $20 \%$ in Bezug auf die OH-Gruppen des ursprünglichen Wang-Harzes bedeutet.

ATR-FTIR: 3430 (breit, O-H), 3024 (C-H, Phenylgruppe), 2920 (C-H), 1727 $(\mathrm{C}=\mathrm{O}), 1600(\mathrm{C}=\mathrm{C}) \mathrm{cm}^{-1}$.

\subsubsection{Polymerisation}

Eine Lösung, bestehend aus Vinylacetat und Toluol, wurde mittels dreimaligem "freeze-pump-and-thaw" an einer Hochvakuumpumpe (Edwards, EXC 120) entgast. Das Gemisch wurde in eine mit Argon befüllte Handschuhbox (Braun, Lab Master 130) eingeschleust. In der Box wurden AIBN als Initiator (ca. $3.3 \mathrm{mmol} \cdot \mathrm{L}^{-1}$ ) und gegebenenfalls das freie Xanthat 7 gemischt. Die Lösung wurde in Schraubdeckelgläser, die eine bestimmte Menge von modifiziertem Wang-Harz enthielten, aufgeteilt. Die Gläser wurden mit den entsprechenden gasdichten Teflon-Deckeln in der Box verschlossen.

Die Polymerisation wurde bei Umgebungsdruck in einem temperierten Ölbad bei $60{ }^{\circ} \mathrm{C}$ unter intensiver Rührung durchgeführt. Nach festgelegten Zeiten wurden die Schraubdeckelgläser aus dem Ölbad entfernt und zum Reaktionsabbruch in ein Eisbad gestellt. Eine kleine Menge der Polymerisationslösung wurde zur Bestimmung des Monomerumsatzes mit Hilfe der NMR-Technik verwendet. Die restliche Polymerisationslösung wurde unter vermindertem Druck getrocknet, um Monomer 
und Toluol zu entfernen. Der Monomerumsatz wurde zusätzlich durch Gravimetrie bestimmt.

Die entstehe Feststoffmischung wurde in einer Soxhlet-Apparatur mit Dichlormethan 12 Stunden lang gewaschen, um das restliche AIBN zu entfernen und um freies Polymer von dem modifizierten Wang-Harz zu trennen. Die Molmassenverteilungen des freien Polymers wurden mittels Gelpermeationschromatographie (GPC) aufgenommen. Das modifizierte Wang-Harz, das das gebundene Polyvinylacetat trägt, wurde im Vakuum getrocknet.

\subsubsection{Abspaltung des gebundenen Polymers}

Das erhaltene modifizierte Wang-Harz wurde in eine mit Argon befüllte Handschuhbox mit einer Lösung von $0.5 \mathrm{~mol} \cdot \mathrm{L}^{-1}$ AIBN bzw. TAPA als Radikalquelle in Toluol, welches vorher mittels dreimaligem "freeze-pump-and-thaw" entgast wurde, gelöst. Die Suspension wurde bei $80{ }^{\circ} \mathrm{C}$ bei Einsatz von AIBN bzw. $130{ }^{\circ} \mathrm{C}$ bei TAPA zwei Stunden lang erhitzt. Nachdem das Reaktionsgemisch abgekühlt war, wurde das Wang-Harz abfiltriert und das erhaltene Filtrat mittels GPC analysiert.

\subsection{Ergebnisse und Diskussion}

\subsubsection{Lösungsmitteleinfluss}

Polystyrol-Harze, wie z. B. Merrifield- oder Wang-Harze, besitzen die Fähigkeit, in unpolaren Lösungsmitteln wie Toluol, Dichlormethan und Tetrahydrofuran um ein Mehrfaches des Trockenvolumens zu quellen. Da sich die eingeführten Xanthate nicht nur an der äußeren Oberfläche, sondern hauptsächlich an der inneren Oberfläche der Harz-Partikel befinden, ist es zwingend notwendig, Lösungsmittel mit guten Quelleigenschaften zu verwenden. Andernfalls können die Reagenzien nicht in das Harz diffundieren und die gebundenen Xanthate erreichen. Polare Lösungsmittel wie Alkohole und Wasser, in denen das Harz schlecht quillt oder verklumpt, sind daher wenig geeignet.

Wegen der Polarität des Vinylacetats kann das modifizierte Wang-Harz, das ein vernetztes Polystyrol ist, darin nicht aufquellen. Deshalb muss ein geeigne- 
tes Lösungsmittel gewählt werden, damit das am Wang-Harz gebundene Xanthat mit der Reaktionslösung in Kontakt treten kann. Außerdem soll das Lösungsmittel die Viskosität der Polymerisationsmischung niedrig halten und darf die MADIXPolymerisation von Vinylacetat nicht signifikant beeinträchtigen.

Toluol erfüllt die geforderten Lösungsmitteleigenschaften. Da sich das Polystyrol gut in Toluol löst, kann das Wang-Harz entsprechend gut darin aufquellen. Außerdem kann das Toluol als Lösungsmittel für die MADIX-Polymerisation eingesetzt werden. Sowohl die Kontrolle der Molmasse als auch die kleine Polydispersität bleiben bis zu einem Monomerumsatz von 90 \% erhalten. Der Einfluss von Toluol auf die Molmassenverteilung durch Transferreaktion ist gering und kann vernachlässigt werden, wenn der Anteil des Toluols an der gesamten Polymerisationsmischung nicht übermäßig groß ist.

Um eine gut kontrollierte Polymerisation durchzuführen, sollte jedoch der Anteil des Lösungsmittel gering wie möglich sein bzw. sollte der Anteil des Monomers möglich groß sein. Abbildung 5.4 zeigt eine Auftragung des Zahlenmittels der Molmassen $M_{\mathrm{n}}$ und Polydispersität PDI des freien Polymers gegen das Massenverhältnis von Monomer zu gesamter Reaktionslösung für die heterogene MADIXPolymerisation von Vinylacetat.

Bei einem hohen Monomer-Massenanteil von 95 \% kann das Wang-Harz wegen der zu geringen Toluolmenge nicht aufquellen. Folglich findet die Polymerisation ohne das gebundene Xanthat statt, was eine konventionelle radikalische Polymerisation bedeutet. Das Polymer aus dieser Polymerisation hat daher einen typisch hohen Wert für die Molmasse und eine Polydispersität von 2.0.

Bei der Verringerung des Monomermassenanteil auf $75 \%$ kann hingegen ein großer Teil des Wang-Harzes in der Reaktionslösung verteilen. Das an Wang-Harz gebundene Xanthat kann in die Polymerisation von Vinylacetat eingreifen. Die mittlere Molmasse des Polymers wird folglich deutlich kleiner. Auch die Polydispersität wird kleiner, ist aber größer als 1.5. Da Teile des eingesetzten Wang-Harzes sichtbar an der Glaswand hafteten und sich nicht in der Reaktionslösung befanden, wurde der Toluolanteil weiter erhöht.

Bei einem Massenanteil des Monomers von ca. 50 \% verkleinert sich die mittlere Molmasse des gebildeten freien Polymers weiter, weil sich das modifizierte Wang- 


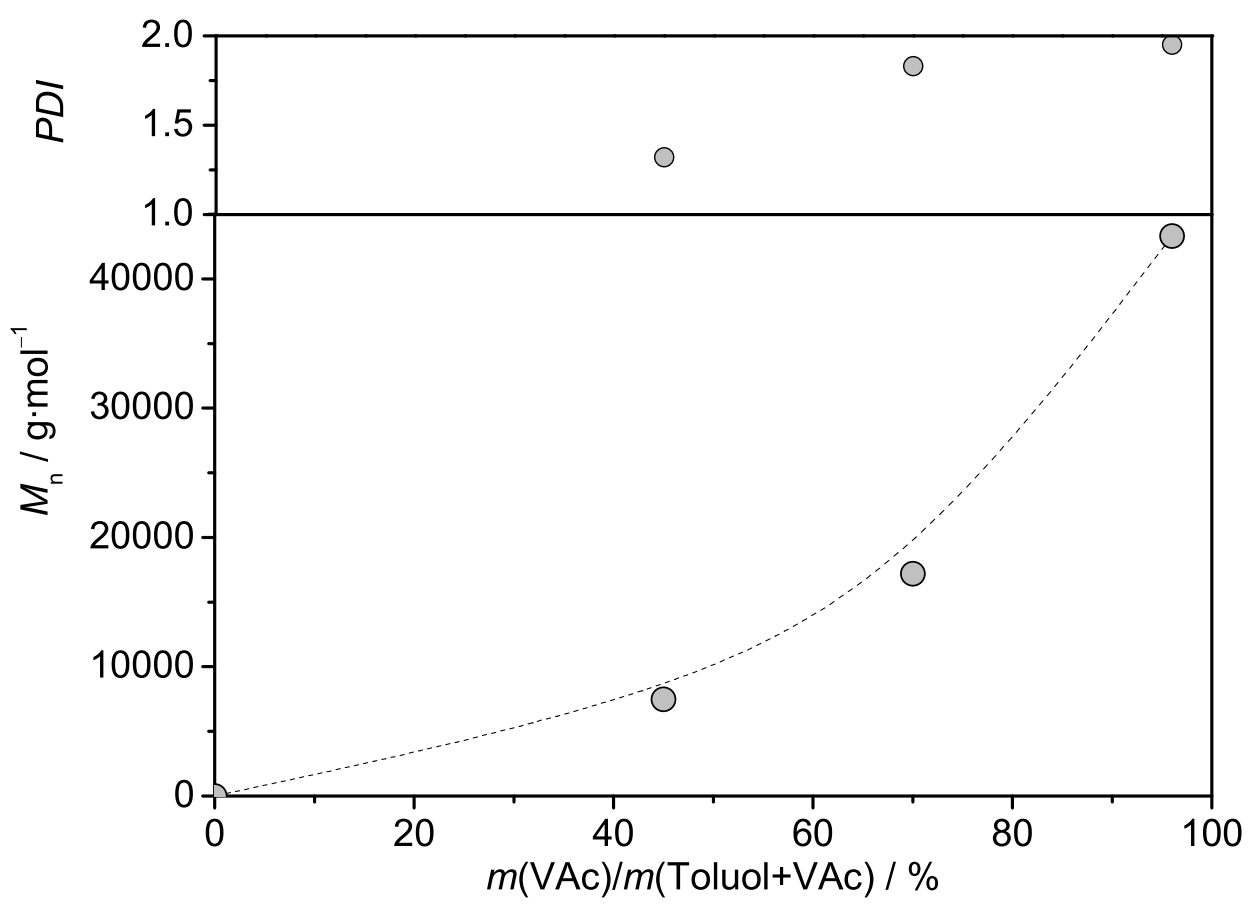

Abbildung 5.4: Auftragung des Zahlenmittels der Molmassen $M_{\mathrm{n}}$ und Polydispersität PDI des freien Polymers gegen das Massenverhältnis von Monomer zur gesamten Reaktionslösung für die heterogene MADIX-Polymerisation von Vinylacetat bei $60{ }^{\circ} \mathrm{C}$, mit AIBN $\left(3.3 \cdot 10^{-3} \mathrm{~mol} \cdot \mathrm{L}^{-1}\right)$ und modifiziertem Wang-Harz (Massenanteil $5 \%$ ). Die gestrichene Kurve ist die Leitlinie.

Harz in diesem Fall vollständig in der Reaktionslösung verteilt hat. Dass die Polydispersität etwas kleiner als 1.5 ist, ist der sichere Beweis dafür, dass eine erfolgreiche heterogene MADIX-Polymerisation statt gefunden hat.

Der optimale Toluolanteil beträgt für die MADIX-Polymerisation von Vinylacetat mit an Wang-Harz gebundenem Xanthat somit 50 \% der gesamten Reaktionslösung. Ein niedrigerer Toluolanteil verhindert die vollständige Löslichkeit des modifizierten Wang-Harzes. Ein höherer Toluolanteil ist unvorteilhaft, da die Transferreaktion zum Lösungsmittel dadurch begünstigt wird, während die Polymerisationsgeschwindigkeit verlangsamt wird, was eine Verschlechterung der Polymerisationskontrolle bedeutet.

Die im Folgenden vorgestellten Polymerisationen wurden, falls nicht anders er- 
wähnt, mit einem Toluolmassenanteil von $50 \%$ durchgeführt. Außerdem wurde eine relativ niedrige AIBN-Konzentration von $3.3 \mathrm{mmol} / \mathrm{L}$ gewählt, um bei einer angemessenen Reaktionszeit den Anteil des toten Polymers, der direkt proportional zu der Menge des zerfallenen Initiators ist, möglichst gering zu halten.

\subsubsection{Einfluß der Partikelkonzentration von Wang-Harz}

Eine Möglichkeit der RAFT-Polymerisationstechnik ist die Molmasse des polymeren Produktes zu verändern, welches durch Variation der Konzentration des eingesetzten RAFT-Agens gelingt. Bei gleichem Monomerumsatz ist die mittlere Molmasse indirekt proportional zu der Konzentration des RAFT-Agens, da die Anzahl der Polymerketten, bei Vernachlässigung des geringen toten Polymers, mit der Anzahl der RAFT-Moleküle identisch ist.

Abbildung 5.5 zeigt den Einfluss unterschiedlicher Mengen des gebundenen Xanthats bzw. der Partikelkonzentration des modifizierten Wang-Harzes auf die Molmasse und die Polydispersität des freien Polymers. Wie erwartet, sinkt die mittlere Molmasse mit steigender Menge des modifizierten Wang-Harzes.

Ein Massenanteil des modifizierten Wang-Harzes von $2.5 \%$ erscheint für eine erfolgreiche MADIX-Polymerisation zu niedrig, weil die Polydispersität mit einem Wert von 1.9 auf eine breite Molmassenverteilung, und damit auf eine schlechte Kontrolle, hindeutet. Bei einem Massenanteil des modifizierten Wang-Harzes von $5 \%$ und höher ist die Polymerisation relativ gut kontrolliert, wie man leicht an der Polydispersität erkennen kann. Dabei muss man bedenken, dass dies die Molmassenverteilungen von freiem Polymer sind. Wie in Kapitel 3 bereits gezeigt wurde, hat das freie Polymer eine deutlich breitere Molmassenverteilung als das gebundene Polymer (siehe Abbildung 3.6).

Eine Polydispersität von 1.5 ist für das freie Polymer erstaunlich niedrig. Um auszuschließen, dass sich ungebundenes RAFT-Agens in den Poren des Wang-Harzes befindet, wurde das modifizierte Wang-Harz noch einmal intensiv mit Toluol und Dichlormethan gewaschen. Nach dem Trocknen in Vakuum wurde die Polymerisation unter gleichen Bedingungen wiederholt. Es entstand ein Polymer mit gleichen Eigenschaften wie vor dem zusätzlichen Waschgang. Durch dieses Experiment kann man ausschließen, dass das modifizierte Wang-Harz noch freies RAFT-Agens enthält. 


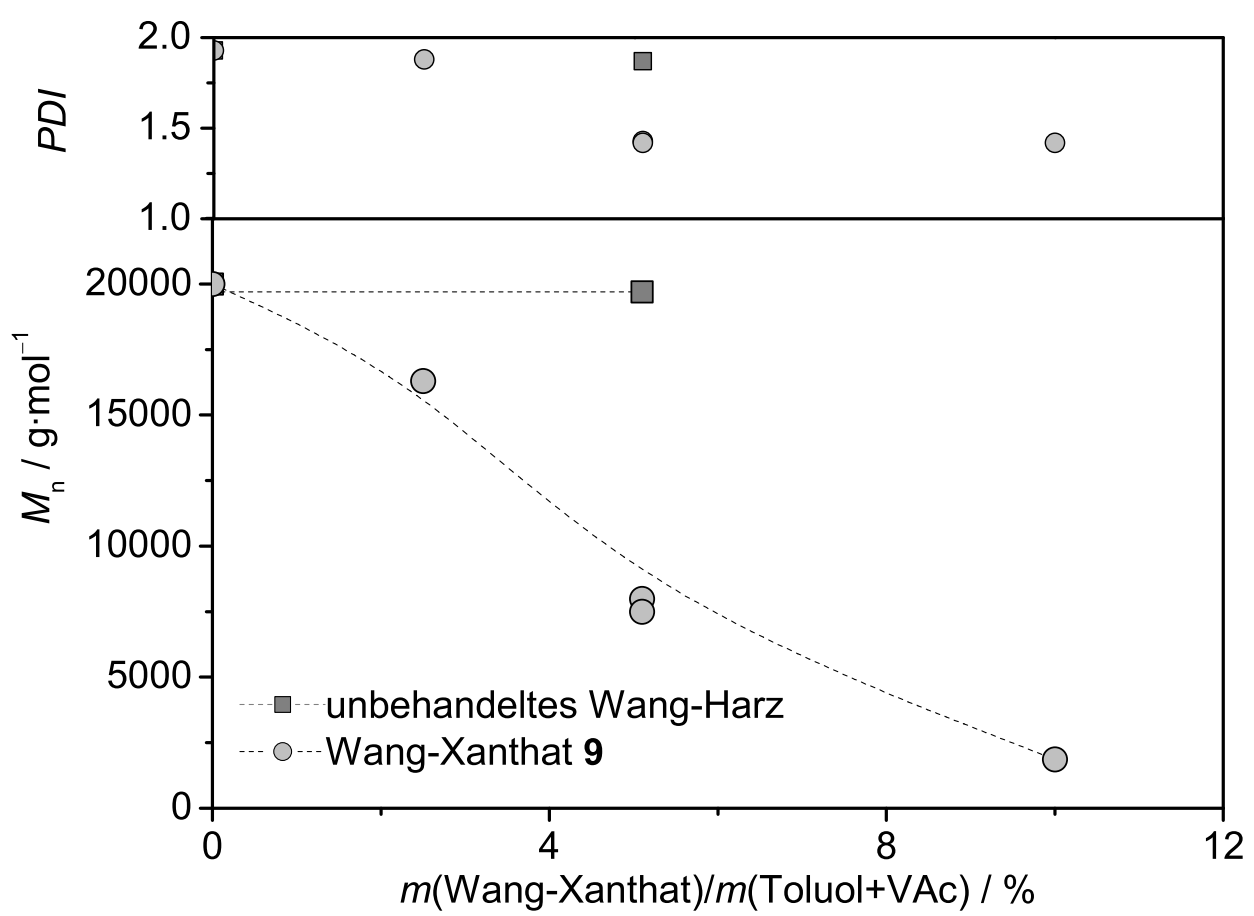

Abbildung 5.5: Auftragung des Zahlenmittels der Molmassen $M_{\mathrm{n}}$ und Polydispersität PDI des freien Polymers gegen das Massenverhältnis von dem modifizierten Wang-Harz 9 zur gesamten Reaktionslösung für die Polymerisation von Vinylacetat bei $60{ }^{\circ} \mathrm{C}$, mit AIBN als Initiator $\left(3.3 \cdot 10^{-3} \mathrm{~mol} \cdot \mathrm{L}^{-1}\right)$. Die Reaktionszeit betrug jeweils 24 Stunden.

Außerdem zeigt dieses Experiment eine gute Reproduzierbarkeit.

Abbildung 5.5 enthält zudem noch den wichtigen Beweis dafür, dass das unbehandelte Wang-Harz keinen signifikanten Einfluss auf die Polymerisation von Vinylacetat hat. Die mittlere Molmasse und die Polydispersität bleiben beim Hinzufügen des unbehandelten Wang-Harzes so gut wie unverändert.

Die folgenden Polymerisationen wurden, falls nicht anders erwähnt, mit einem Massenanteil des modifizierten Wang-Harzes von ca. $5 \%$ durchgeführt. Basierend auf der Beladungsdichte des gebundenen Xanthats (0.20 mmol/g Wang-Harz) wurde die Konzentration des Xanthats auf $11.1 \mathrm{mmol} / \mathrm{L}$ Reaktionslösung bestimmt. Bei einem Vinylacetat-Massenanteil von $50 \%$ und einer AIBN-Konzentration von $3.3 \mathrm{mmol} / \mathrm{L}$ konnte das folgende Stoffmengenverhältnis bestimmt werden. 
Monomer : gebundenem Xanthat : Initiator $=1000: 2.0: 0.55$

Dieses Stoffmengenverhältnis stellt erfahrungsgemäß die typische RAFT-Agens-Konzentration einer gut kontrollierten homogenen Lösungspolymerisation dar.

\subsubsection{Bestimmungsmethoden für den Monomermsatz}

Für die sinnvolle Auswertung der RAFT-Polymerisation ist eine korrekte Bestimmung des Monomerumsatzes unerlässlich. In dieser Arbeit wurden zwei einfach durchführbare Standardmethoden, Gravimetrie und NMR-Spektroskopie, zur Bestimmung des Umsatzes eingesetzt und verglichen.

Die gravimetrische Bestimmung des Monomerumsatzes erfolgt durch die folgende Gleichung:

$$
X_{\text {gravi }}=\frac{m_{\mathrm{PVAc}}}{m_{\mathrm{VAc}}}=\frac{m_{\mathrm{P}}-m_{\mathrm{Wang}-\mathrm{Xanthat}}}{m_{\mathrm{VAc}}}
$$

Dabei ist $m_{\mathrm{PVAc}}$ die Masse des gebildeten Polyvinylacetat, $m_{\mathrm{VAc}}$ die Masse des eingesetzten Monomers, $m_{\mathrm{P}}$ die Masse des gesamten polymeren Endprodukts und $m_{\text {Wang-Xanthat }}$ die Masse des verwendeten modifizierten Wang-Harzes. Die gravimetrisch bestimmten Umsätze basieren auf der Massenbilanz und sind im Rahmen der experimentellen Unsicherheit absolut verlässlich.

Die ${ }^{1} \mathrm{H}-\mathrm{NMR}-$ Spektroskopie ist eine einfache und genaue Methode, um den Monomerumsatz zu verfolgen. Dabei wird von der Reaktionsmischung direkt nach der Polymerisation ein ${ }^{1} \mathrm{H}-\mathrm{NMR}-S p e k t r u m$ gemessen. Aus dem Flächenverhältnis charakteristischer Signale des Monomers zu einer Signalgruppe, welche sowohl im Monomer als auch im Polymer vorliegt, lässt sich der Umsatz wie folgt bestimmen.

$$
X_{\mathrm{NMR}}=1-\frac{I_{\mathrm{B}}}{I_{\mathrm{A}}}
$$

Dabei ist $I_{\mathrm{B}}$ das Intergral über den Signalen der drei Wasserstoffatome ${ }^{\mathrm{B}} \mathrm{H}$, die der Anzahl des restlichen Monomers nach der Polymerisation entsprechen, und $I_{\mathrm{A}}$ das Intergral über den Signalen der drei Wasserstoffatome ${ }^{\mathrm{A}} \mathrm{H}$, die der Anzahl der gesamten Vinylacetat-Einheiten in der Polymerisationslösung entsprechen (siehe Abbildung 5.6). Diese Signale lassen sich gut von den Signalen des Toluols unterscheiden, sodass eine eindeutige Zuordnung der Signale und die genaue Bestimmung der Intergrale problemlos möglich sind. 

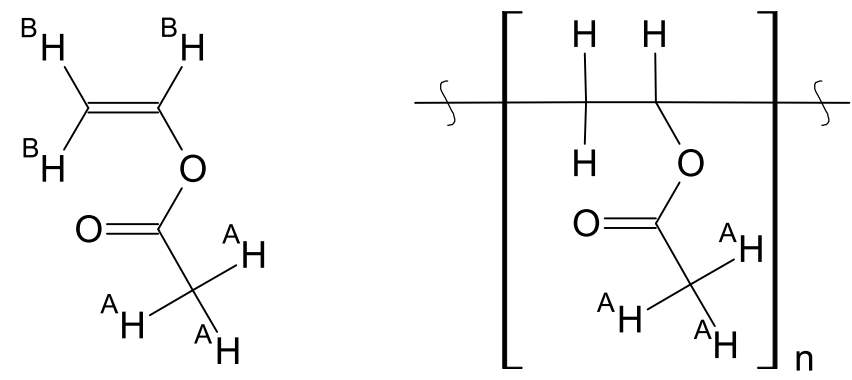

Abbildung 5.6: Struktur von Vinylacetat und Polyvinylacetat. Zur Berechnung von Monomerumsatz mit Hilfe der NMR-Spektrometrie wird die Fläche der Signalserien von ${ }^{A} \mathrm{H}$ und ${ }^{\mathrm{B}} \mathrm{H}$ verwendet.

Die Ergebnisse aus den beiden Methoden sind in Abbildung 5.7 dargestellt. Wie man leicht sieht, gibt es einen großen Unterschied zwischen den Ergebnissen der beiden Bestimmungsmethoden. Während die beiden Methoden vergleichbare Umsatzwerte für die homogene Lösungspolymerisation liefern, ist der Unterschied in der heterogenen Polymerisation umso größer, je höher die Partikelkonzentration des modifizierten Wang-Harzes ist.

Die unterschiedlichen Ergebnisse der beiden Methoden für den Monomerumsatz lassen sich wie folgt erklären: Die in Lösung routinemäßig durchgeführte NMRSpektroskopie liefert aufgrund starker Signalverbreitungen durch die gehinderte Beweglichkeit der Substrate kaum erfassbare Signale für das gebundene Polyvinylacetat. Die NMR-Technik kann nur die Wasserstoffatome des freien Polyvinylacetats detektieren. Nur das freie Polymer, das in deuteriertem Chloroform löslich ist, gibt entsprechende ${ }^{1} \mathrm{H}-\mathrm{NMR}-$ Signale.

Zur Überprüfung dieser Aussage wurde das Wang-Harz, das nichts anderes als funktionalisiertes und vernetztes Polystyrol ist, in deuteriertem Chloroform NMRspektrokopisch gemessen. Das entstehende ${ }^{1} \mathrm{H}-\mathrm{NMR}$-Spektrum enthält kein Signal des Polystyrols. Dieser Befund bekräftigt die obige Erklärung.

Das Fehlen der NMR-Signale des gebundenen Polyvinylacetats verursacht einen systematischen Fehler bei der Bestimmung des Monomerumsatzes nach der NMRMethode. Je höher die Partikelkonzentration des modifizierten Wang-Harzes ist, desto höher ist die Anzahl des gebundenen Polyvinylacetats und desto größer ist 


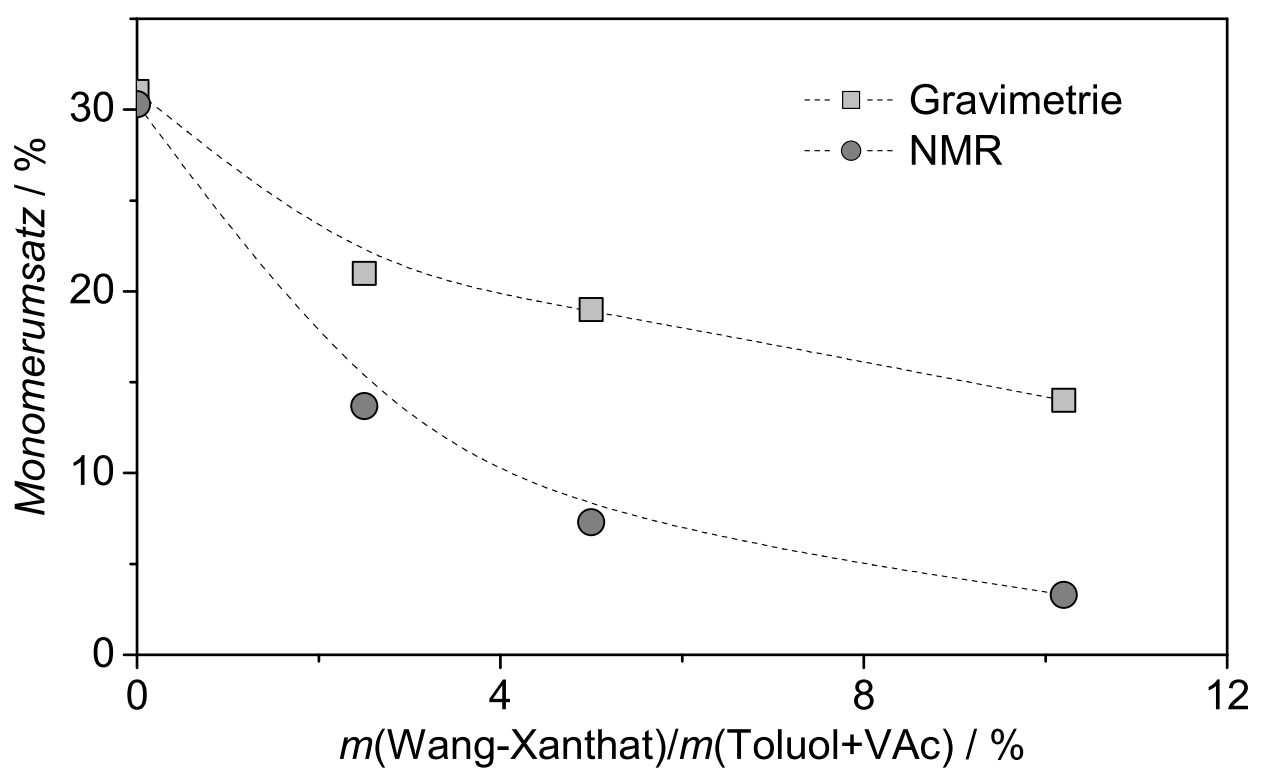

Abbildung 5.7: Auftragung des Monomerumsatzes gegen das Massenverhältnis von modifiziertem Wang-Harz 9 zur gesamten Reaktionslösung für die heterogene MADIXPolymerisation von Vinylacetat bei $60{ }^{\circ} \mathrm{C}$, mit AIBN $\left(3.3 \cdot 10^{-3} \mathrm{~mol} \cdot \mathrm{L}^{-1}\right)$. Die Reaktionszeit betrug jeweils 24 Stunden. Der Monomerumsatz wurde mittels Gravimetrie und NMR-Spektroskopie bestimmt.

somit der Unterschied bzw. der Fehler von $X_{\mathrm{NMR}}$ zu dem gravimetrisch bestimmten Monomerumsatz $X_{\text {gravi }}$.

Die Differenz der Umsatzwerte aus der gravimetrischen und NMR-spektroskopischen Bestimmung stellt die Menge des gebundenen Polyvinylacetats dar und kann folglich dafür ausgenutzt werden, um den Massenanteil $f_{\text {gebunden }}$ des gebundenen Polyvinylacetats zu berechnen:

$$
f_{\text {gebunden }}=\frac{m_{\text {gebunden }}}{m_{\text {gebunden }}+m_{\text {frei }}}=1-\frac{X_{\mathrm{NMR}}}{X_{\text {gravi }}}
$$

In dieser Gleichung ist $m_{\text {gebunden }}$ die Masse des gebundenen Polyvinylacetats und $m_{\text {frei }}$ die Masse des freien Polymers. Die so erhaltenen Massenanteile des gebundenen Polyvinylacetats sind in Tabelle 5.1 aufgelistet. 


\begin{tabular}{cc}
\hline Massenanteil von $\mathbf{9} / \%$ & $f_{\text {gebunden }} / \%$ \\
\hline 2.5 & 34.7 \\
5.0 & 61.7 \\
10.2 & 76.3 \\
\hline
\end{tabular}

Tabelle 5.1: Massenanteil des gebundenen Polyvinylacetats in Abhängigkeit vom Massenanteil des eingesetzten modifizierten Wang-Harzes 9.

Der Massenanteil des gebundenen Polyvinylacetats $f_{\text {gebunden }}$ steigt mit zunehmendem Massenanteil des modifizierten Wang-Harzes. Dieser Befund passt qualitativ gut mit dem RAFT-Mechanismus zusammen. Auch wenn der Anteil des freien Polymers für eine ideale RAFT-Polymerisation relativ groß ist, ist er beim Vergleich mit der heterogenen RAFT-Polymerisation, bei der das gebundene Cumyldithiobenzoat (siehe Kapitel 3) über die stabilisierende Z-Gruppe chemisch an einem Substrat festgebunden ist, doch relativ niedrig.

Der vergleichsweise niedrige Anteil an freiem Polymer setzt eine gute Erreichbarkeit des gebundenen Xanthats voraus. Außerdem bildet das aufgequollene modifizierte Wang-Harz ein Netzwerk, in dessen gesamtem freiem Volumen sich die Polymerisationslösung befindet. Damit ist eine gleichmäßige Verteilung des am Wang-Harz gebundenen Xanthats gewährleistet.

Für die weitere Auswertung wurde der Monomerumsatz, der mit der gravimetrischen Methode bestimmt wurde, herangezogen, da er verlässlicher erscheint.

\subsubsection{Abspaltung des gebundenen Polyvinylacetats}

Um die Molmassenverteilung des gebundenen Polyvinylacetats zu charakterisieren, wurden drei verschiedene Techniken zur Abspaltung der gebundenen Polymerketten verwendet:

1. Aminolyse mit n-Hexylamin: Diese Methode ist eine nukleophile Substitution unter milden Reaktionsbedingungen. Dabei verliert das gebundene Xanthat seine chemische Funktionalität, was eine Wiederverwendung des Katalysators ausschließt [72]. 

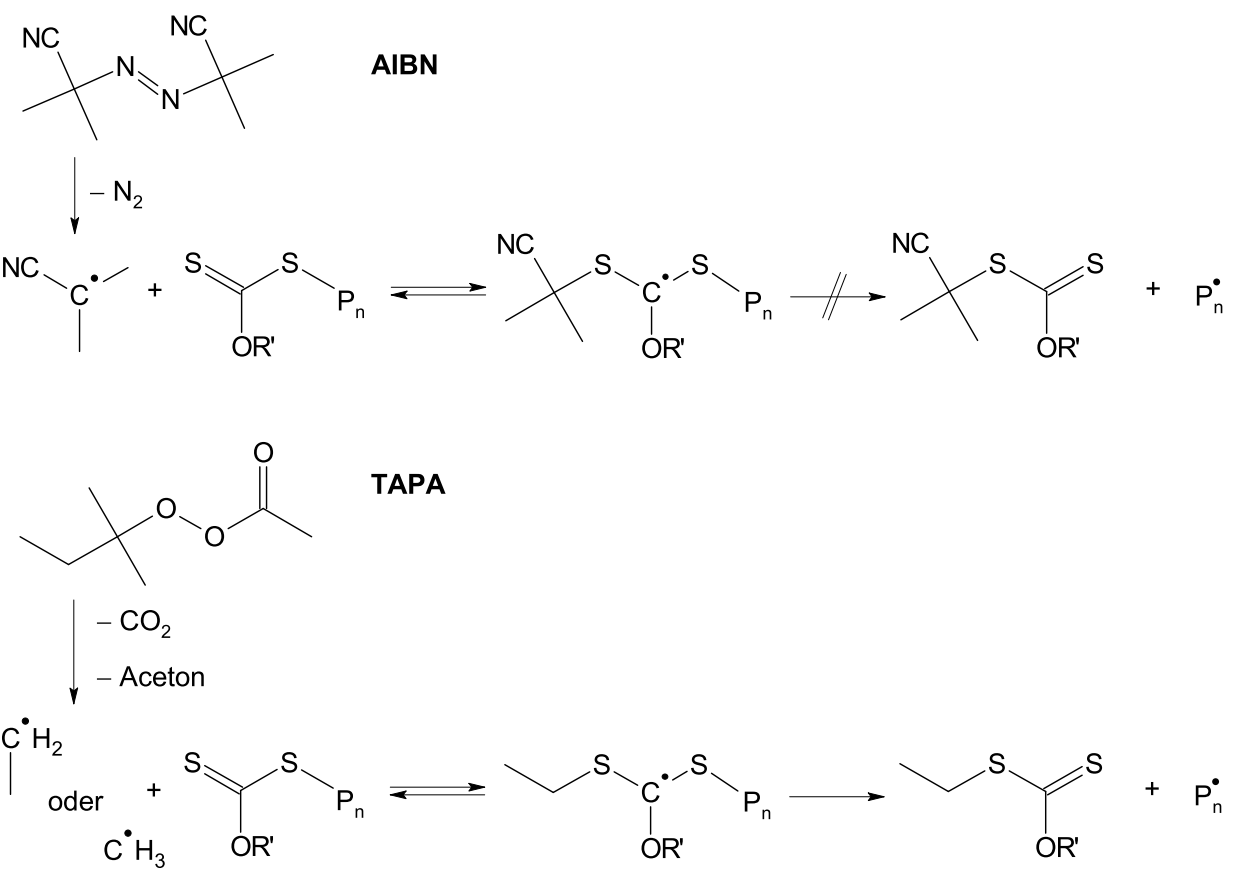

Abbildung 5.8: Mechanismus der Abspaltung der Xanthat-Endgruppe des Polyvinylacetats mit einer überschüssigen Menge von AIBN bzw. von TAPA.

2. Einmaliger Transferschritt mit einer überschüssigen Menge von AIBN: Diese bereits in Kapitel 3 beschriebene Methode ermöglicht im Prinzip die Wiedergewinnung des gebundenen RAFT-Agens. Doch unter Verwendung von AIBN gelingt die Methode nicht, weil das Polyvinylradikal als Abgangsgruppe im Gegensatz zum Cyanoisopropylradikal zu schlecht ist (siehe Abbildung 5.8).

3. Einmaliger Transferschritt mit tert-Amylperoxyacetat (TAPA): Diese Methode funktioniert mechanistisch entsprechend der zweiten Methode. Anstatt AIBN wird TAPA als Quelle für primäre Radikale verwendet. TAPA ist ein primärer Peroxyester bezüglich des $\alpha$-Kohlenstoffatoms der Säureseite. Bei dem Zerfall von TAPA entstehen reaktive primäre Radikale [116], die in der Lage sind, die Xanthat-Endgruppe des Polyvinylacetats abzutrennen (siehe Abbildung 5.8).

Um das an Wang-Harz gebundene Polyvinylacetat abzuspalten hat sich die dritte Methode als am besten geeignet erwiesen. Ein Vergleich der Molmassenverteilung des freien und gebundenen Polymers, das mit einer überschüssigen Menge von TAPA 


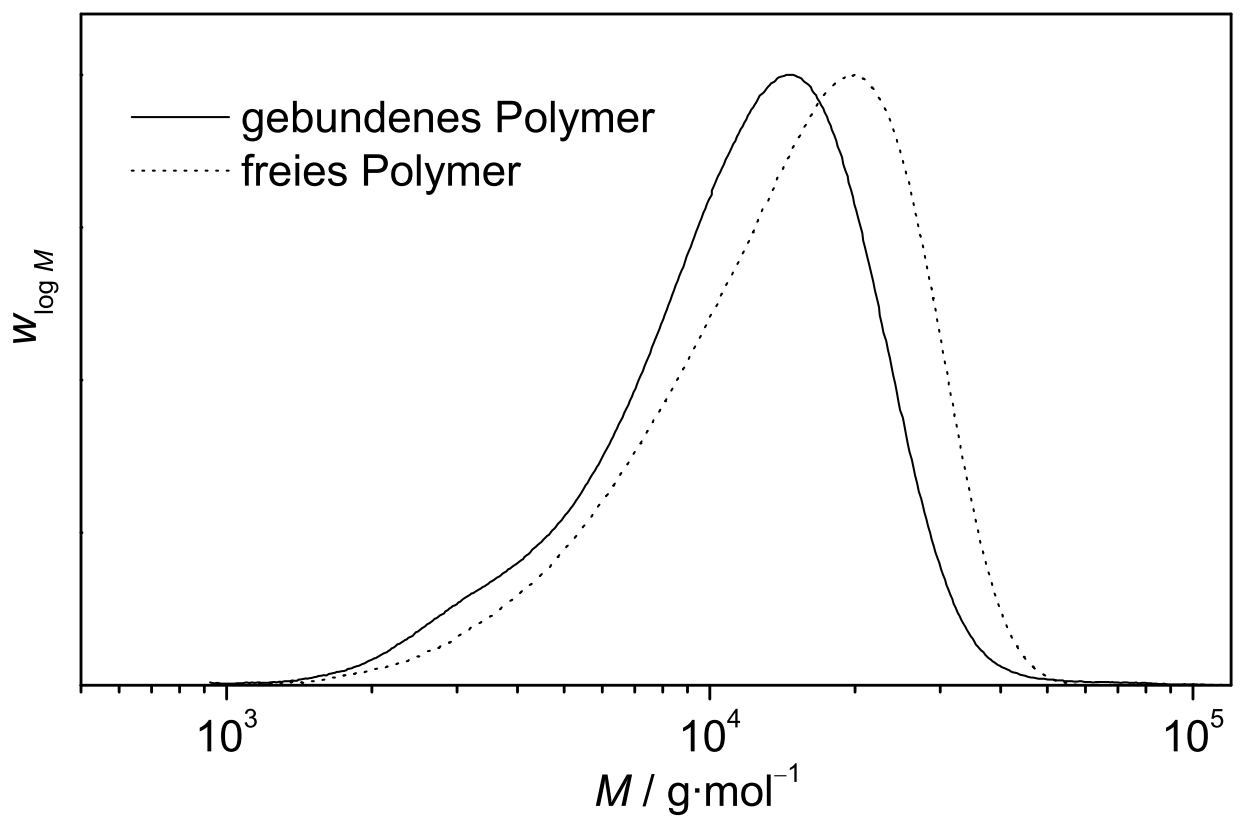

Abbildung 5.9: Molmassenverteilung des Polyvinylacetats, das nach einem Monomerumsatz von $19 \%$ in der heterogenen MADIX-Polymerisation bei $60{ }^{\circ} \mathrm{C}$ in Toluol entsteht. Die Polymerisation wird mit einem Stoffmengenverhältnis von $n$ (Monomer) $: n$ (gebundenes Xanthat $): n(\mathrm{AIBN})=1000: 2.0: 0.55$ durchgeführt. Die gepunktete Linie zeigt das freie Polymer, das in der flüssigen Phase gebildet wurde. Die durchgezogene Linie gehört zu dem gebundenen Polymer, das nach der Polymerisation mit Hilfe von TAPA von dem Wang-Harz abgespalten wurde.

durch einen einmaligen Transferschritt vom Wang-Harz abgespalten wurde, ist in Abbildung 5.9 dargestellt.

Um auszuschließen, dass sich noch freies Polyvinylacetat in den Poren von modifiziertem Wang-Harz befindet, wurde das Wang-Harz in einer Soxhlet-Apparatur mit Dichlormethan 12 Stunden lang gewaschen. Danach wurde der Rückstand eine Stunde bei Zimmertemperatur in THF gerührt. Nach dem Filtrieren wurde das THF eingeengt und eine GPC-Analyse durchgeführt. Da der RI-Detektor der GPCAnalyse sehr empfindlich ist, können dadurch kleinste Mengen von freiem Polymer, falls vorhanden, nachgewiesen werden. Es hat sich herausgestellt, dass nach dem Waschen in der Soxhlet-Apparatur das modifizierte Wang-Harz kein freies Polyvi- 
nylacetat mehr enthielt.

Es wurde außerdem überprüft, ob TAPA mit dem polymeren Träger reagiert und das polymere Netzwerk in einzelne Polymerketten spaltet. Dazu wurde unbehandeltes Wang-Harz auf gleicher Weise mit TAPA behandelt, wie es zur Abspaltung des gebundenen Polyvinylacetats erforderlich war. Nach der Reaktion und Filtration enthielt das Filtrat kein mittels RI-Detektor nachweisbares Polymer.

Das Polymer, das sich nach der Reaktion mit TAPA in der flüssigen Phase befindet, ist das gebundene Polyvinylacetat aus der MADIX-Polymerisation mit dem an Wang-Harz gebundenen Xanthat. Im nächsten Abschnitt werden die Molmasse sowie deren Verteilung des freien und gebundenen Polymers systematisch untersucht und verglichen.

\subsubsection{Einfluss von zusätzlichem freiem Xanthat}

Die systematische Untersuchung der Entwicklung der mittleren Molmasse mit dem Monomerumsatz ist eine gute Methode, um wichtige Kenntnisse über die Effizienz einer kontrollierten Polymerisation zu erlangen. Dabei kann man sich ein umfassendes Bild der kontrollierten Polymerisation von der Anfangsphase bis zum vollständigen Monomerumsatz machen.

Abbildung 5.10 zeigt die Entwicklung der Molmasse und der Polydispersität in Abhängigkeit vom Monomerumsatz für die heterogene MADIX-Polymerisation von Vinylacetat mit dem an Wang-Harz gebundenen Xanthat. Dabei sind sowohl die Werte des freien Polymers als auch die des gebundenen Polymers, das mit einer überschüssigen TAPA-Menge vom modifizierten Wang-Harz abgespalten wurde, aufgetragen.

Die theoretische mittlere Molmasse $M_{\mathrm{n}}^{\text {theo }}$ wird für gesamtes Polymer wie folgt berechnet:

$$
M_{\mathrm{n}}^{\text {theo }}=\frac{m_{\mathrm{M}} \cdot U_{\mathrm{M}}}{n_{\text {gebunden }}+n_{\text {frei }}+2 \cdot n_{\mathrm{I}} \bullet}
$$

In dieser Gleichung sind $m_{\mathrm{M}}$ die Masse des Monomers, $U_{\mathrm{M}}$ der fraktionelle Monomerumsatz, $n_{\text {gebunden }}$ die Stoffmenge von gebundenem Xanthats, $n_{\text {frei }}$ die Stoffmenge von freiem Xanthats und $n_{\mathrm{I}} \bullet$ die Stoffmenge von effektivem primärem Radikal 


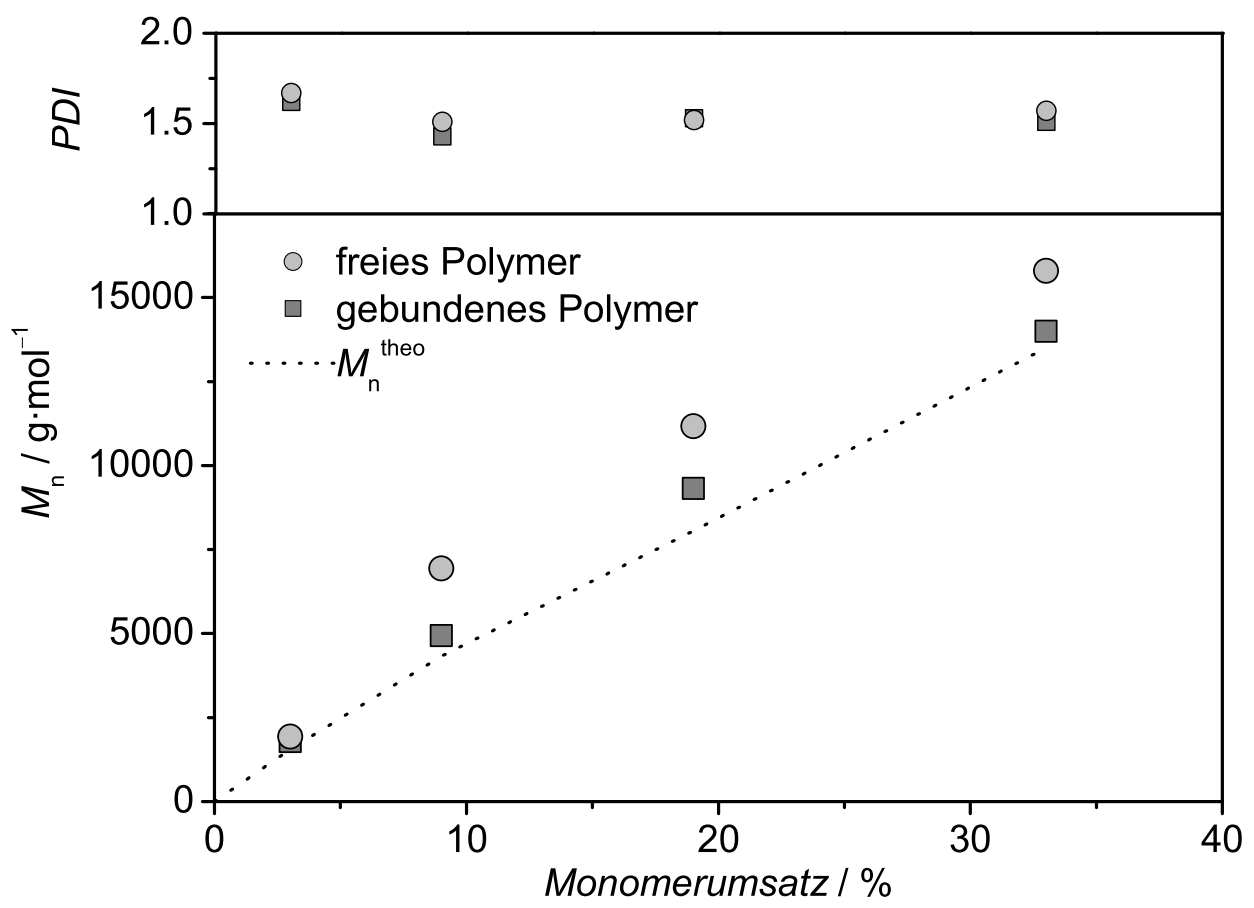

Abbildung 5.10: Auftragung des Zahlenmittels der Molmassenverteilung $M_{\mathrm{n}}$ und der Polydispersität $P D I$ gegen den Monomerumsatz für die heterogene MADIX-Polymerisation von Vinylacetat bei $60{ }^{\circ} \mathrm{C}$. Die Polymerisation wurde mit einem Stoffmengenverhältnis von $n$ (Monomer) $: n$ (gebundenes Xanthat) $: n($ AIBN $)=1000: 2.0: 0.55$ in Toluol durchgeführt. Die gepunktete Linie stellt die berechneten theoretischen Molmassen für gesamtes Polymer dar.

aus dem Initiatorszerfall. Die Terminierung verläuft bei Vinylacetat über Kombination, wobei aus zwei propagierenden Radikalen eine Polymerkette entsteht. Die Übertragungsreaktion auf das Lösungsmittel ist langsam und kann vernachlässigt werden.

Wie man in Abbildung 5.10 sieht, nehmen die mittleren Molmassen des freien und gebundenen Polymers linear mit dem Monomerumsatz zu und stimmen mit der theoretischen Molmasse $M_{\mathrm{n}}^{\text {theo }}$ überein. Diese beiden Beobachtungen weisen auf eine erfolgreiche MADIX-Polymerisation mit dem gebundenen Xanthat hin.

Für die Polymerisationsreihe, bei der kein freies Xanthat hinzu gegeben wurde, 
ist das am Wang-Harz gebundenen Xanthat das einzige Kontrollagens. Da das Xanthat durch die stabilisierende Z-Gruppe chemisch an der Oberfläche des polymeren Trägers verankert ist, bildet das gebundene Polymer die lebende polymere Fraktion, während das freie Polymer die tote Fraktion darstellt.

Beim Vergleich der Molmassen des freien Polymers mit der des gebundenen Polymers fällt auf, dass die freien Polymerketten signifikant länger sind als die gebundenen Ketten. Für diese Beobachtung gibt es zwei mögliche Erklärung:

1. Die Polymerisation läuft zu einem kleinen Anteil unkontrolliert in der flüssigen Phase ab. Denn die Erreichbarkeit der Xanthatgruppe für die propagierenden Radikale ist relativ schlecht im Falle der Verankerung des Xanthates über die stabilisierende Z-Gruppe. Die schlechte Erreichbarkeit der Xanthatgruppe wird mit der steigenden Kettenlänge des Polymers wegen zunehmender sterischer Abschirmung verstärkt. Sie wird außerdem mit dem steigenden Monomerumsatz weiterverstärkt, weil die Viskosität mit steigendem Monomerumsatz stark zunimmt, was eine bedeutend schlechtere Beweglichkeit der Polymerketten verursacht.

2. Das freie Polyvinylacetat wird aus der Terminierung zweier Makroradikalen gebildet. Da die Terminierung von Vinylacetat über Kombination verläuft, entstehen dadurch längere Polymerketten. Bei der Abspaltung der gebundenen Polymerketten mit primären Radikalen können die abgespalteten Makroradikale nur mit den primären Radikalen terminieren. Terminierungen zwischen zwei Makroradikale sind wegen des großen Überschusses an primären Radikalen unwahrscheinlich. Dadurch entstehen im Vergleich mit freiem Polymer kürzere Polymerketten.

Die Polydispersität des freien und gebundenen Polymers liegt um den Wert von 1.5 (siehe Abbildung 5.10). Die Werte unterhalb von 1.5 liefern einen sicheren Beweis dafür, dass die Polymerisation kontrolliert abgelaufen ist. Dennoch sind die erhaltenen Molmassenverteilungen für eine gut kontrollierte MADIX-Polymerisation sehr breit. Auch dieser Befund liegt in der relativ schlechten Erreichbarkeit der Xanthatgruppe an der Oberfläche des Wang-Harzes begründet.

Um die Kontrolle der heterogenen MADIX-Polymerisation zu erhöhen, und damit eine engere Molmassenverteilung des polymeren Produktes zu erhalten, wurde 


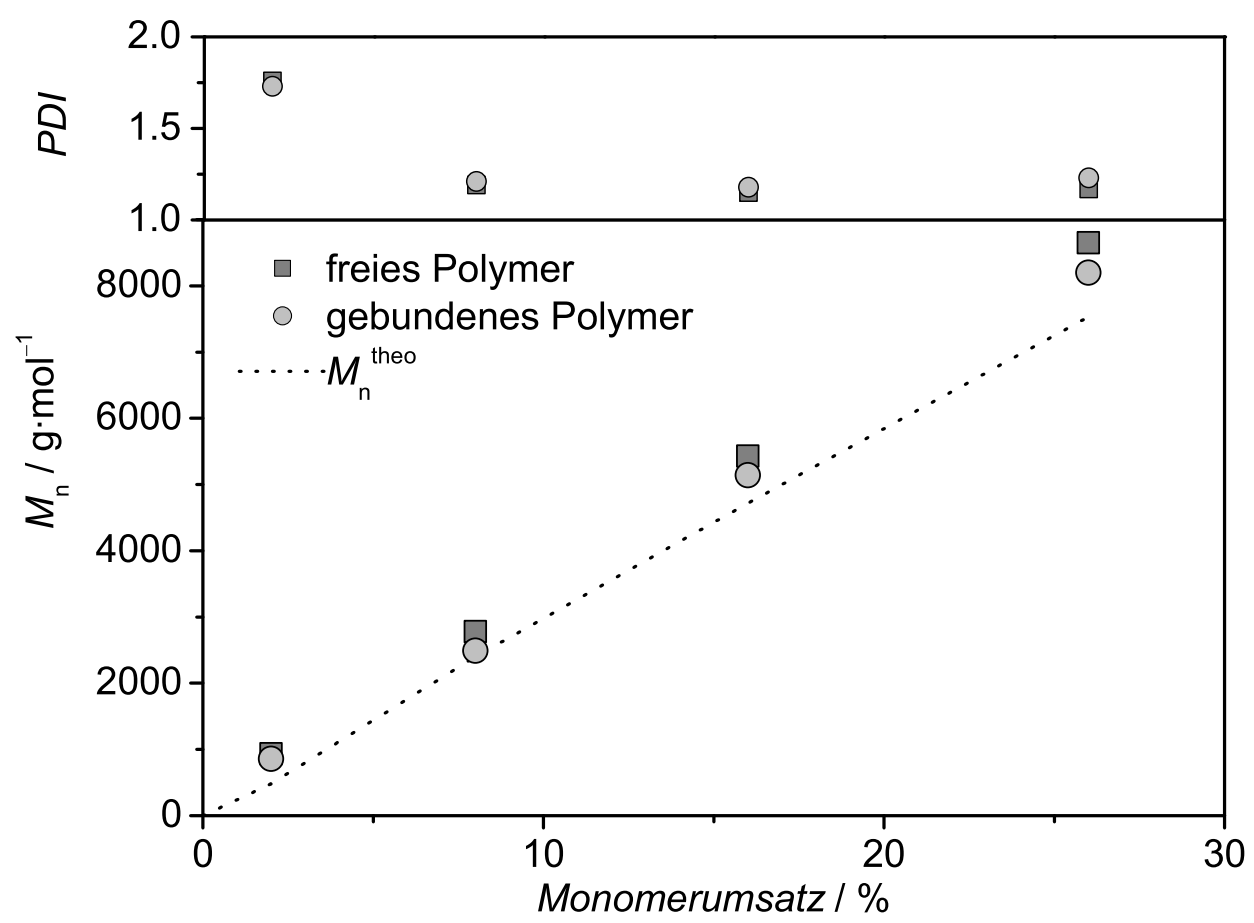

Abbildung 5.11: Auftragung des Zahlenmittels der Molmassenverteilung $M_{\mathrm{n}}$ und der Polydispersität PDI gegen den Monomerumsatz für die heterogene MADIX-Polymerisation von Vinylacetat bei $60{ }^{\circ} \mathrm{C}$ mit zusätzlichem freiem Xanthat 7. Die Polymerisation wurde mit einem Stoffmengenverhältnis von $n$ (Monomer) $: n$ (gebundenes Xanthat) $: n$ (freies Xanthat) $: n($ AIBN $)=1000: 2.0: 0.88: 0.55$ durchgeführt. Die gepunktete Linie stellt die theoretischen Molmassen für gesamtes Polymer dar.

zusätzlich zu dem gebundenen Xanthat freies, ungebundenes Xanthat $\mathbf{7}$ in die Polymerisationsmischung gegeben. Die Ergebnisse dieser Polymerisationsreihe sind in Abbildung 5.11 gezeigt.

Wie erwartet, haben sowohl das freie als auch das gebundene Polyvinylacetat eine vergleichsweise enge Molmassenverteilung. Die Polydispersität beträgt, abgesehen von der Anfangsphase der Polymerisation, zwischen 1.1 und 1.2. Das freie Xanthat hat eine sehr gute Kontrolle der heterogenen MADIX-Polymerisation von Vinylacetat ermöglicht.

Durch die zusätzlichen Xanthatmoleküle erhöht sich entsprechend die Anzahl 
der Polymerketten. Dadurch ist die mittlere Molmasse im Vergleich zu der Polymerisationsreihe, die ohne freies Xanthat durchgeführt wurde, deutlich kleiner. Der sterische Abschirmungseffekt wird durch die kürzeren Polymerketten verringert, was die Erreichbarkeit der Xanthatgruppe an der Oberfläche erhöht. Das wird als ein Hauptgrund für die gute Kontrolle angesehen.

Die mittlere Molmasse des Polymers hängt direkt von der Konzentration des kontrollierenden Agens ab, wie man aus Gleichung 5.4 ableiten kann. Ein großer Unterschied der lokalen Konzentration in der Polymerisationsmischung, wie es bei einer heterogenen Reaktion inhärent ist, führt unweigerlich zur Verbreiterung der Molmassenverteilung des entstehenden Polymers. Das freie Xanthat kann sich gut in der Reaktionsmischung verteilen und lokale Konzentrationsunterschiede ausgleichen, wodurch die Kettenlänge des Polymers einheitlicher wird.

Bei der heterogenen kontrollierten Polymerisation findet immer ein gewisser Anteil der Reaktion in der flüssigen Phase statt. Das freie Xanthat unterbindet durch seine Anwesenheit die unkontrollierte Polymerisation in der flüssigen Phase und ermöglicht damit, dass das gesamte Polymerisationssystem in einer kontrollierten Art und Weise reagiert. Dadurch sind die mittleren Molmassen des freien und gebundenen Polymers fast identisch. Außerdem stimmt die theoretische Molmasse $M_{\mathrm{n}}^{\text {theo }}$ mit dem experimentellen Werte sehr gut überein. Dieser Befund bestätigt die Annahme, dass die Transferreaktion vernachlässigbar gering ist und dass alle eingesetzten Xanthatmoleküle in die heterogene MADIX-Polymerisation von Vinylacetat eingreifen.

\subsubsection{Direkte Charakterisierung des gebundenen Polyvinyl- acetats}

Um das gebundene Polyvinylacetat direkt zu charakterisieren, ohne die umständliche Prozedur zur Abspaltung mit TAPA durchzuführen, wurden im Folgenden zwei Methoden angewendet:

1. ATR-FTIR-Spektroskopie (Attenuated Total Reflection²- Fourier Transformation Infrarotspektroskopie): Mit der ATR-Technik lassen sich Proben unter-

\footnotetext{
${ }^{2}$ Attenuated Total Reflection $=$ abgeschwächte Totalreflexion
} 
suchen, die für Transmissions-Messungen nicht geeignet sind, z. B. Pasten, Kunststofffasern oder Beschichtungen von Oberflächen. Da aber bereits der polymere Träger ein sehr bandenreiches IR-Spektrum besitzt, werden viele Schwingungen der gebundenen Verbindungen überlagert. Das verwendete Polystyrol-Harz besitzt jedoch Bereiche geringer Absorption, so dass für die Charakterisierung z. B. die Banden von Carbonyl-Gruppen geeignet sind.

2. TGA (Thermogravimetrische Analyse) : TGA ist ein Verfahren der Thermoanalyse, bei dem die Masse oder Massenänderung einer Probe in Abhängigkeit von der Temperatur unter Verwendung eines kontrollierten Temperatur-Programms gemessen wird.

\section{ATR-FTIR-Spektroskopie}

In Abbildung 5.12 sind die FTIR-Spektren, die mit der ATR-Technik aufgenommen wurden, von dem unbehandelten Wang-Harz und dem modifizierten Wang-Harz vor und nach der heterogenen MADIX-Polymerisation von Vinylacetat gezeigt. Das Spektrum von dem unbehandelten Wang-Harz ist, abgesehen von dem breiten Signal der O-H-Schwingung bei $3400 \mathrm{~cm}^{-1}$, vergleichbar mit dem Spektrum von Polystyrol.

Das Spektrum des modifizierten Wang-Harzes, das vor der MADIX-Polymerisation nur die Xanthatgruppe an seinem Oberfläche enthält, hat im Vergleich mit dem Spektrum vom unbehandelten Wang-Harz ein zusätzliches Signal bei $1727 \mathrm{~cm}^{-1}$. Da Carbonylgruppen eine starke charakteristische Absorption in diesem Wellenzahlbereich zeigen, darf man annehmen, dass im modifizierten Wang-Harz Estergruppen vorliegen. Dieser Befund stimmt mit der Struktur des gebundenen Xanthates überein, dessen Abgangsgruppe eine Estergruppe enthält. Andere Schwingungen des Xanthates liefern nur schwache Absorptionssignale, die von den Banden des WangHarzes überlagert werden.

Das Spektrum des modifizierten Wang-Harzes, das nach der MADIX-Polymerisation das gebundene Polyvinylacetat mit einer mittleren Molmasse von $1.8 \mathrm{~kg} / \mathrm{mol}$ enthält, zeigt beim Vergleich mit den zwei anderen Spektren ein hohes Signal bei $1727 \mathrm{~cm}^{-1}$. Auch dieses Signal weist auf Carbonylgruppe hin. Dies stimmt mit der Struktur des Polyvinylacetats überein, das carbonylhaltige Estergruppen enthält. 


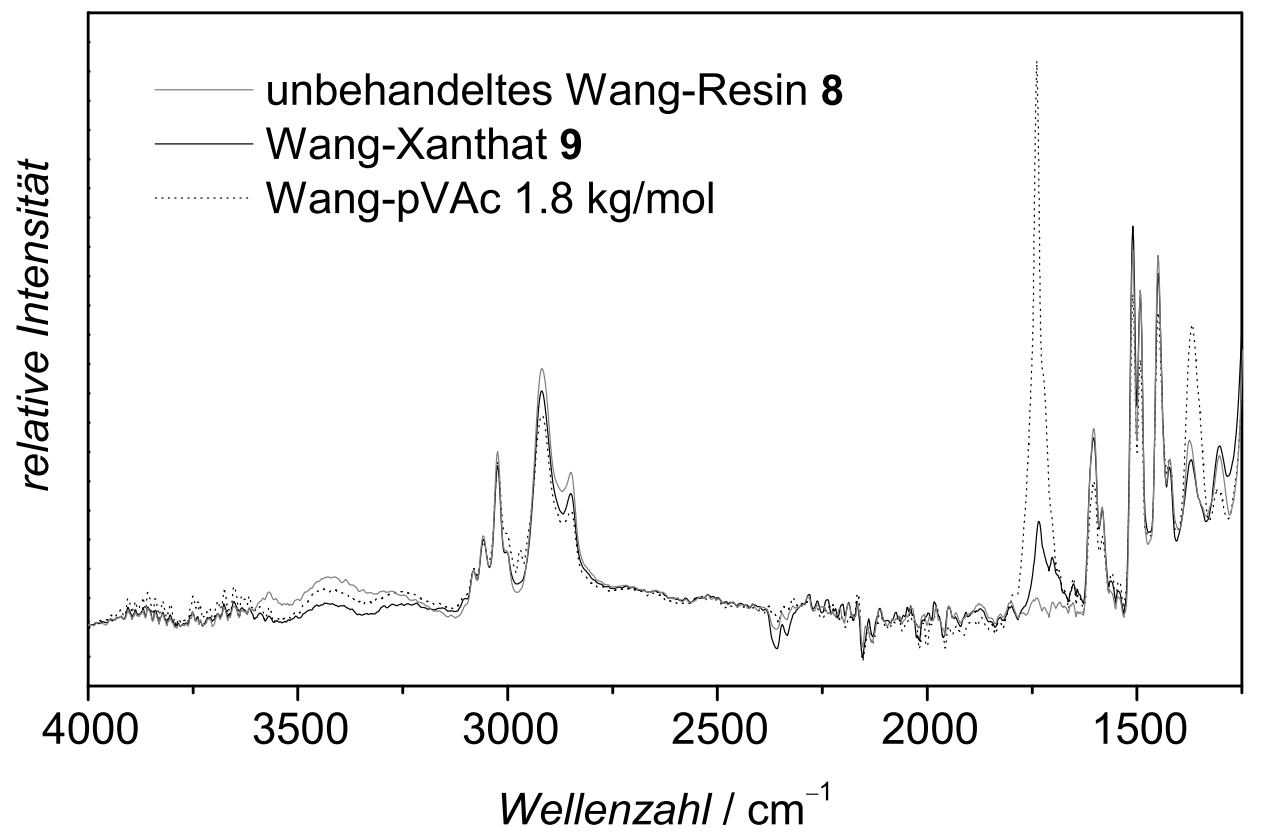

Abbildung 5.12: ATR-FTIR-Spektren von dem unbehandelten Wang-Harz und dem modifizierten Wang-Harz vor und nach der heterogenen MADIX-Polymerisation von Vinylacetat. Die Spektren wurden über die C-H-Schwingungsbanden bei $3000 \mathrm{~cm}^{-1}$ des PolystyrolGrundgerüstes normiert.

Das Polyvinylacetat mit der mittleren Molmasse von $1.8 \mathrm{~kg} / \mathrm{mol}$ besteht aus ca. 20 monomere Einheiten, die jeweils eine Carbonylgruppe enthalten. Das gebundene Polymer hat also ca. 20-mal mehr Carbonylgruppe als das gebundene Xanthat. Folglich sollte das Signal bei $1727 \mathrm{~cm}^{-1}$ nach der Polymerisation ca. 20-mal größer sein als das Signal vor der Polymerisation. Diese quantitative Annahme wird, wie man in Abbildung 5.12 sehen kann, annähernd erfüllt.

Eine genaue quantitative Analyse des gebundenen Polymers, mit der die mittlere Molmasse direkt gemessen werden könnte, setzt eine sorgfältige Kalibrierungen und gleich bleibende Bedingungen des Messexperiments voraus. Die weitere Entwicklung und Optimierung dieser quantitativen Analysemethode würde den Rahmen dieser Arbeit sprengen und wird deshalb hier nicht weiter verfolgt. 


\section{Thermogravimetrische Analyse}

Die zweite verwendete Methode, die das am Wang-Harz gebundene Polyvinylacetat direkt charakterisiert, ist die thermogravimetrische Analyse (TGA). Abbildung 5.13 zeigt die gemessenen TGA-Kurven von dem unbehandelten Wang-Harz, dem Polyvinylacetat, das mittels homogener MADIX-Polymerisation synthetisiert wurde, und dem modifizierten Wang-Harz nach der heterogenen MADIX-Polymerisation von Vinylacetat. Vor dem TGA-Experiment wurden die Proben einen Tag lang bei $40{ }^{\circ} \mathrm{C}$ unter Vakuum sorgfältig von möglichem Lösungsmittel befreit.

Das unbehandelte Wang-Harz zersetzt sich bei ca. $300{ }^{\circ} \mathrm{C}$, was der typischen Zersetzungstemperatur von Polystyrol entspricht. Bis zu dieser Temperatur bleibt die Masse des unbehandelten Wang-Harzes annähernd erhalten. Erst ab einer Temperatur von ca. $300{ }^{\circ} \mathrm{C}$ nimmt die Masse des Wang-Harzes durch Zersetzung signifikant ab. Bei der Endtemperatur von $500{ }^{\circ} \mathrm{C}$ bleibt ein Restgehalt von ca. $15 \%$ der Anfangsmasse vom Wang-Harz zurück.

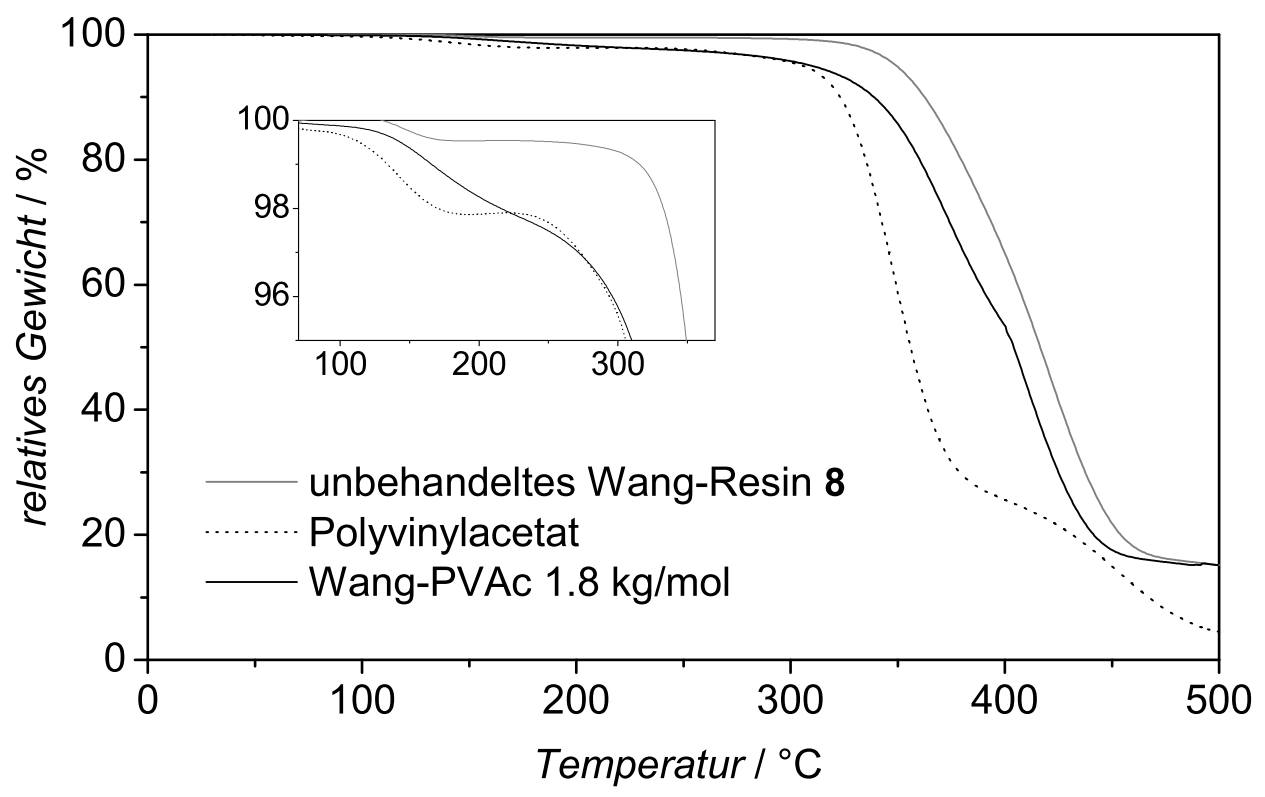

Abbildung 5.13: TGA-Kurve des unbehandelten Wang-Harzes, des Polyvinylacetats, das aus einer homogenen MADIX-Polymerisation gebildet wurde, und des modifizierten WangHarzes nach der heterogenen MADIX-Polymerisation von Vinylacetat. Das kleine Diagramm stellt die Vergrößerung der TGA-Kurve in der Anfangsphase dar. 
Das Polyvinylacetat, das mittels homogener MADIX-Polymerisation synthetisiert wurde, zeigt eine erste Zersetzung bei $120^{\circ} \mathrm{C}$ und verliert ca. $2 \%$ seiner Masse. Dieser Massenanteil gehört zu den Xanthat-Gruppen, die bei dieser Temperatur nicht mehr stabil sind [117]. Das eigentliche Polyvinylacetat ist erst ab $250{ }^{\circ} \mathrm{C}$ instabil. Die Zersetzungstemperatur vom Polyvinylacetat ist um $50{ }^{\circ} \mathrm{C}$ niedriger als die vom Polystyrol bzw. vom Wang-Harz.

Das modifizierte Wang-Harz ${ }^{3}$, das vor der MADIX-Polymerisation die XanthatGruppe enthält, zeigt im Temperaturbereich von $120-170{ }^{\circ} \mathrm{C}$ einen Massenverlust von $3 \%$. Dieser Wert entspricht dem Massenanteil des gebundenen Xanthats und stimmt mit dem Ergebnis aus der Elementaranalyse überein. Eine genaue Berechnung der Beladungsdichte des gebundenen Xanthates aus der TGA-Kurve setzt jedoch die Kenntnis des detaillierten Zerfallsmechanismus des Xanthats voraus, was nicht gegeben ist. Der weitere Verlauf der TGA-Kurve gleicht der Kurve des unbehandelten Wang-Harzes.

Das modifizierte Wang-Harz mit gebundenem Polyvinylacetat ist im Prinzip ein Blockcopolymer von Polystyrol und Polyvinylacetat mit einer Xanthatgruppe, die sich zwischen den beiden Blöcken befindet. Folglich besteht die TGA-Kurve aus einer Überlagerung der Zerfälle vom Xanthat, vom Polyvinylacetat und vom Polystyrol. Mit zunehmender Temperatur zersetzt sich zuerst das Xanthat bei ca. $130{ }^{\circ} \mathrm{C}$, dann das Polyvinylacetat bei $250{ }^{\circ} \mathrm{C}$ gefolgt von dem polymeren Träger Polystyrol, das erst ab einer Temperatur von $300{ }^{\circ} \mathrm{C}$ instabil wird.

Das Massenverhältnis von Polystyrol-Grundgerüst und gebundenem Polyvinylacetat kann aus der mittleren Molmasse des gebundenen Polymers $(1.8 \mathrm{~kg} / \mathrm{mol})$ und der Beladungsdichte des Xanthats $(0.20 \mathrm{mmol} / \mathrm{g}$ Harz $)$ berechnet werden:

$$
m_{\mathrm{PSty}}: m_{\mathrm{PVAc}} \approx 3: 1
$$

Da die Zerfälle von Polyvinylacetat und Polystyrol sich in der TGA-Kurve stark überlagern, ist eine eindeutige quantitative Bestimmung des Massenanteils vom gebundenen Polyvinylacetat nicht möglich. Qualitativ hingegen kann die TGA-Technik das Xanthat und das Polyvinylacetat, die an der Oberfläche von Wang-Harz gebunden sind, eindeutig nachweisen.

\footnotetext{
${ }^{3}$ Seine TGA-Kurve wird aus Gründen der Übersichtlichkeit in der Abbildung 5.13 nicht gezeigt.
} 


\subsection{Schlussfolgerung}

Die Synthese eines gebundenen Xanthats an hydroxyhaltigem Wang-Harz als Trägersubstanz wurde detailliert untersucht. Es wurde gefunden, dass das Wang-Harz als polymerer Träger für die heterogene RAFT-Polymerisation vor allem dann gut geeignet ist, wenn es in der Reaktionslösung gut aufquellen kann.

Die radikalische heterogene MADIX-Polymerisation von Vinylacetat wurde bei der Verwendung des an Wang-Harz gebundenen Xanthats bezüglich seiner Molmasse erfolgreich kontrolliert. Die Fixierung des RAFT-Agens an das feste Wang-Harz ermöglicht eine effektive Trennung des lebenden Polymers von dem toten Polymer, das während der RAFT-Polymerisation gebildet wird.

Die Methode der Immobilisierung des RAFT-Agens über die Z-Gruppe erlaubt die Herstellung von schwefelfreiem Polymermaterial mit kontrollierter Molmasse und bietet einen Weg zur Wiedergewinnung des gebundenen Xanthats. Mit überschüssigen Mengen von dem Initiator TAPA konnte dazu das gebundene Polyvinylacetat vom polymeren Träger abgespalten werden und das gebundene Xanthat wurde dadurch wiedergewonnen.

Zusätzlich zu dem gebundenen Xanthat kann zur effizienteren Kontrolle der polymeren Oberflächenmodifizierung des Wang-Harzes ein freies Xanthat in die Polymerisationsmischung zugesetzt werden, um engere und definiertere Molmassenverteilungen des polymeren Produktes zu erhalten.

Für die quantitative Bestimmung des Massenanteils des gebundenen Polyvinylacetats wurde ein neues Konzept entwickelt, in dem Monomerumsatz-Bestimmungen über Gravimetrie sowie NMR-Spektroskopie kombiniert wurden.

Für eine direkte Charakterisierung des modifizierten Wang-Harzes wurden die ATR-FTIR-Spektroskopie und die thermogravimetrische Analyse erfolgreich eingesetzt. Beide Methoden bestätigen qualitativ die erwartete Strukturen des an WangHarz gebundenen Xanthats und sowie des gebundenen Polyvinylacetats. 



\section{Teil B}

\section{Homogene}

\section{RAFT-Polymerisation}





\section{Kapitel 6}

\section{Polymerisation mit Dithiobenzoesäure}

In Kapitel 3 wurde gezeigt, dass die an der Silica-Oberfläche gebundene Dithiobenzoesäure die Polymerisation von Methylmethacrylat und Styrol kontrollieren kann. In diesem Kapitel wird ein detaillierter Mechanismus der Polymerisation von Methylmethacrylat mit Dithiobenzoesäure vorgestellt.

\subsection{Einführung}

Dithiosäuren bilden eine effektive Klasse von RAFT-Agenzien, die zuerst von Bai et al. [84, 118] am Beispiel von Dithiobenzoesäure (DTBA, dithiobenzoic acid, 1, siehe Abbildung 6.1) in der Literatur beschrieben wurde. Diese Substanz kontrolliert die radikalischen Polymerisationen von Styrol, Methylmethacrylat (MMA, 2) und Methylacrylat, welche zu Polymeren mit zunehmender Molmasse bei steigendem Monomerumsatz führen. Die erhaltenen Polymere haben kleine Polydispersitäten, der niedrigste berichtete PDI beträgt 1.08.

Die Dithiobenzoesäure ist als RAFT-Agens besonders attraktiv, weil sie sich leicht und kostengünstig herstellen lässt. Sie ist die Zwischenstufe für die Synthese vieler effektiver RAFT-Agenzien wie z. B. Cumyldithiobenzoat und 2-Cyanoisoprop2-yldithiobenzoat. Durch eine Verringerung der Anzahl an Reaktionsschritten in der Synthese kann die Ausbeute an RAFT-Agenzien stark erhöht und die Durchführung vereinfacht werden. 
Plummer et al. [119] zeigten, dass die Dithiobenzoesäure als Verunreinigung in dem oft benutzten RAFT-Agens Cumyldithiobenzoat ein Hauptgrund für die charakteristische Induktion und Retardierung der Polymerisation von Styrol, Methylacrylat und 2-Hydroxyethylmethacrylat ist. Über die Ursache und die kinetische Beschreibung der Induktion und Retardierung werden intensive Diskussionen in der aktuellen Literatur geführt. Die Erforschung des Mechanismus der RAFTPolymerisation mit Dithiobenzoesäure kann einen kleinen Teil zu dieser Debatte leisten.

Der genaue Mechanismus der kontrollierten radikalischen Polymerisation mit DTBA war in der Literatur umstritten. DTBA kann als ein gewöhnliches RAFTAgens mit einem Wasserstoff als Abgangsgruppe R missverstanden werden. Da ein Wasserstoffradikal sehr instabil ist, ist diese Erklärung nicht plausibel. Bai et al. [84] haben schon früh erkannt, dass das Wasserstoffatom keine geeignete Abgangsgruppe ist und dass der RAFT-Prozess nicht durch einen Addition-Fragmentierung-Schritt im Vorgleichgewicht abläuft. Sie schlugen einen Mechanismus vor, in dem sich ein Dithioester durch die Addition von DTBA an die Doppelbindung des Monomers in situ bildet. Der gebildete Dithioester trägt das Monomer als gute Abgangsgruppe und ist daher ein effektives RAFT-Agens. Diese Hypothese wird durch die Strukturanalyse des gebildeten Polymers bestätigt. Das Polymer trägt die gleiche DithioesterEndgruppe wie in einer klassischen RAFT-Polymerisation [120].

Solch eine in situ Bildung von RAFT-Agens funktioniert nur mit Doppelbindungen, die - wie im Fall des Styrols - elektronengebende Substituenten haben. Ein effektives RAFT-Agens wird demnach durch die Addition von DTBA an die Doppelbindung des Monomers nach einem Markovnikov-Mechanismus erzeugt [121]. Die Addition von DTBA zu der Doppelbindung mit einem elektronenziehenden Substituenten - wie bei Methylacrylat oder MMA - erfolgt aber nach dem MichaelMechanismus [122]. Der entstehende Dithioester hat einen primären Kohlenstoff als Abgangsgruppe (siehe Abbildung 6.1). Dieser Dithioester ist deshalb als RAFTAgens ungeeignet, da ein primärer Kohlenstoff ohne die Resonanzstabilisierungsmöglichkeit für das ungepaarte Elektron eine schlechte Abgangsgruppe ist. Dieses Prinzip ist von den Gruppen Duréault [123] und Monteiro [121] detailliert beschrieben und durch Experimente bestätigt worden. 
<smiles>O=C(S)c1ccccc1</smiles>

DTBA 1<smiles>C=C(C)C(=O)OC</smiles>

MMA 2<smiles>COC(=O)C(C)CSC(=S)c1ccccc1</smiles>

3

Abbildung 6.1: Bei der Additionsreaktion von DTBA zu MMA nach dem MichaelMechanismus ergibt sich ein inaktives RAFT-Agens, da die Abspaltung der Gruppe mit einer Radikalfunktionalität am primären Kohlenstoff energetisch ungünstig ist.

Duréault et al. [123] nutzten Phosphor(V)sulfid oder Davy-Reagenz ${ }^{1}$ in einer „One-pot“-Reaktion, um die Polymerisation von Styrol zu kontrollieren. Die Dithiobenzoesäure wird in diesem Fall als Zwischenstufe für die gebildeten RAFTAgenzien betrachtet. Weitere Hinweise für den Mechanismus der Polymerisation von Styrol und Acrylate mit DTBA lieferten Plummer et al. [119]. Sie beobachteten eine ausgeprägte Induktionsperiode von mehreren Stunden bei der Polymerisation bei $60{ }^{\circ} \mathrm{C}$. Die Induktionsperiode ist signifikant länger als die theoretische Zeit für eine einfache Inhibierungsreaktion. Diese Erkenntnis deutet auf einen komplexen Mechanismus in der Anfangsphase der Polymerisation hin. Ähnliche Induktionsperioden wurden von Xu et al. [124] beobachtet. Diese Forschungsgruppe studierte die MMAPolymerisation mit der Dithiobenzoesäure, welche durch thermische Zersetzung von Dithiobenzoat in situ gebildet wurde.

In einer detaillierten Studie von Goh et al. [121] wurde die Polymerisation von Methylacrylat und Styrol mit 2-Phenyldithioessigsäure untersucht. Die Autoren wiesen darauf hin, dass die Mechanismen der Bildung des RAFT-Agens sich für die einzelnen Monomere unterscheiden. Für Styrol sei eine Markovnikov-Addition von Phenyldithioessigsäure zu Styrol, die ein effektives RAFT-Agens hervorbringt, der Hauptgrund für die beobachtete Kontrolle der Molmasse. Für Methylacrylat, zu welchem die Phenyldithioessigsäure nach einem Michael-Mechanismus langsam ad-

\footnotetext{
${ }^{1}$ Der systematische Name von Davy-Reagenz ist (2,4-Bis(methylthio)-1,3-dithia-2,4-diphosphetane-2,4-disulfide).
} 
diert, wird ein anderer Mechanismus vorgeschlagen. Dieser Mechanismus enthält einen Transferschritt des propagierenden Radikals zu der Phenyldithioessigsäure. Das propagierende Radikal abstrahiert dabei das Wasserstoffatom von Phenyldithioessigsäure, welche sich zu Phenylmethylcarbothioylsulfanyl-Radikal $\left(\mathrm{Ph}-\mathrm{CH}_{2}-\right.$ $\left.C(=S) S^{\bullet}\right)$ umwandelt. Das neu gebildete Dithio-Radikal soll reinitiieren und anschließend mit einem anderen Radikal terminieren. Die Autoren vermuteten, dass das dabei entstehende Terminierungsprodukt ein effektives RAFT-Agens sei. Sie übersahen jedoch die Tatsache, dass das reinitierende Dithio-Radikal das Monomer an dem weniger substituierten Kohlenstoff der Doppelbindung attackiert. Dadurch hat das Terminierungsprodukt im Prinzip die gleiche Dithioestergruppe wie bei der Michael-Addition $\left(-\mathrm{S}-\mathrm{CH}_{2}-\mathrm{CH}(\mathrm{COOMe})-\right)$. Wegen dieser schlechten Abgangsgruppen sind alle Dithioester, die vom reinitiierenden Dithio-Radikal stammen, unreaktive RAFT-Agenzien.

Ausgehend von der ersten Entdeckung durch Bai et al. [84] und von dem Konzept der Gruppe um Monteiro [121] wird ein detailliertes kinetisches Bild der kontrollierten radikalischen Polymerisation mit Dithiobenzoesäure in diesem Kapitel präsentiert. Weiter wird ein vollständiger Mechanismus für die Bildung von RAFTAgens in der Induktionsphase aufgestellt, der den experimentellen Befund der Induktion und die Endgruppen des gebildeten Polymers plausibel erklärt. Der vorgeschlagene Mechanismus wird außerdem durch kinetische Simulationen getestet. Mit Hilfe dieses Mechanismus wird ein Syntheseprotokoll für die kontrollierte Polymerisation von MMA vorgestellt, welches die Induktionszeit drastisch verringert und die Konzentration des in situ erzeugten RAFT-Agens signifikant erhöht.

\subsection{Experiment}

\subsubsection{Chemikalien}

Dithiobenzoesäure wurde entsprechend der Literatur synthetisiert [76]. Zu einer 30\%igen Lösung von Natriummethanolat (18 g, $100 \mathrm{mmol}$ ) in Methanol werden elementarer Schwefel (3.2 g, $100 \mathrm{mmol}$ ) und über einen Zeitraum von einer Stunde tropfenweise Benzylchlorid (6.3 g, $50 \mathrm{mmol}$ ) hinzugegeben. Die erhaltene braune Lösung wurde über Nacht unter Rückfluss erhitzt. Nachdem die Lösung auf Raumtempera- 
tur abgekühlt war, wurde der weiße Feststoff (Natriumchlorid) abfiltriert und Methanol unter verminderten Druck entfernt. Der verbleibende braune Feststoff wurde in $50 \mathrm{~mL}$ destilliertem Wasser gelöst, und die Lösung wurde fünfmal mit jeweils $20 \mathrm{~mL}$ Diethylether extrahiert. Bei der Zugabe der letzten Volumina Diethylether wurde das Zwei-Phasen-Gemisch mit konzentrierter Salzsäure angesäuert. Die wässrige Phase verlor dabei ihre charakteristische braune Farbe, gleichzeitig färbte sich die etherische Phase violett. Die etherische Phase wurde dreimal mit destilliertem Wasser gewaschen und über Calciumchlorid getrocknet. Nach dem Entfernen des restlichen Ethers unter Vakuum erhielt man die Dithiobenzoesäure als dunkel purpurfarbenes Öl (3.4 g, 22.0 mmol, 44 \%). Die Reinheit von Dithiobenzoesäure wurde durch NMR-Spektroskopie geprüft (Reinheitsgrad $\geq 98 \%$ ).

${ }^{1} \mathrm{H}-\mathrm{NMR}\left(300 \mathrm{MHz}, \mathrm{CDCl}_{3}\right), \delta(\mathrm{ppm}): 5.6(\mathrm{~s}, 1 \mathrm{H},-\mathrm{C}(=\mathrm{S}) \mathrm{SH}), 7.2-8.1(\mathrm{~m}, 5 \mathrm{H}$, $\left.\mathrm{C}_{6} H_{5}\right)$.

${ }^{13} \mathrm{C}-\mathrm{NMR}\left(300 \mathrm{MHz}, \mathrm{CDCl}_{3}\right), \delta(\mathrm{ppm}): 225$ (-C(=S)S), 134 (C1), 133 (C4), 128 $(2 \times \mathrm{C} 2), 127(2 \times \mathrm{C} 3)$.

\subsubsection{Polymerisation}

MMA, Initiator (AIBN oder/und ACCN) mit einer Startkonzentration von ca. 5 $\mathrm{mmol} \cdot \mathrm{L}^{-1}$ und die Dithiobenzoesäure mit einer Startkonzentration zwischen 2.7 und $60 \mathrm{mmol} \cdot \mathrm{L}^{-1}$ wurden vorbereitet und mittels dreimaligem ,freeze-pump-andthaw" an einer Hochvakuumpumpe (Edwards, EXC 120) entgast. Die Polymerisationslösung wurde in eine mit Argon befüllte Handschuhbox (Braun, Lab Master 130) eingeschleust und in Schraubdeckelgläser aufgeteilt. Die Schraubdeckelgläser wurden mit den entsprechenden gasdichten Deckeln in der Handschuhbox verschlossen.

Die Polymerisation wurde bei Umgebungsdruck in einem Thermoblock (Rotilabo, Block Heater H 250) durchgeführt. Die Schraubdeckelgläser wurden in die Bohrungen des Thermoblocks gegeben und nach den angegebenen Zeiten aus dem Thermoblock entfernt und zum Reaktionsabbruch in ein Eisbad gestellt.

Nach der erfolgten Polymerisation wurde der Inhalt jeweils eines Schraubdeckelglases in eine Aluminiumschale zur gravimetrischen Monomerumsatzbestimmung gegeben. Die Polymerproben wurden unter Vakuum bei Zimmertemperatur bis zur Massenkonstanz getrocknet. Die Molmassenverteilung der Polymere erfolgte mittels 
der Gelpermeationschromatographie (GPC). Die Endgruppen der Polymere wurden durch ESI-Massenspektrometrie analysiert.

\subsection{Ergebnisse und Diskussion}

\subsubsection{Induktion und Retardierung}

Die erste Beobachtung bei der Polymerisation von Methylmethacrylat mit Dithiobenzoesäure ist die sehr lange Induktionsphase, in welcher kein merklicher Monomerumsatz stattfindet (siehe Abbildung 6.2). Sie beträgt z. B. bis zu sieben Stunden bei einer DTBA-Konzentration von $10.8 \cdot 10^{-3} \mathrm{~mol} \cdot \mathrm{L}^{-1}$ und AIBN-Konzentration von $4.9 \cdot 10^{-3} \mathrm{~mol} \cdot \mathrm{L}^{-1}$, obwohl eine hohe Initiator-Konzentration in der Größenordnung der DTBA-Konzentration gewählt wurde. Bei einem für die RAFT-Polymerisation typischen Konzentrationsverhältnis von RAFT-Agens : Initiator $=10: 1$ war die Induktionsphase so groß, dass die MMA-Polymerisation nach drei Tagen noch nicht startete.

Bei der RAFT-Polymerisation ist häufig zu beachten, dass am Anfang der Reaktion eine Induktion vorliegt, die durch das Vorgleichgewicht des RAFT-Mechanismus bedingt ist. Für Dithiobenzoesäure muss das Vorgleichgewicht anders ablaufen, weil der Wasserstoff ein sehr instabiles Radikal ist und nicht als eine Abgangsgruppe fungieren kann.

Die Induktionsphase nimmt nahezu proportional mit der DTBA-Konzentration in der Reaktionsmischung zu, wie man in Abbildung 6.3 und Tabelle 6.1 leicht erkennen kann. Die Induktionszeit $t_{\text {ind }}$ ist als Schnittpunkt der Zeit-Achse mit der linearen Anpassung für die Polymerisationsphase nach der Induktion in der UmsatzZeit-Auftragung definiert. Der absolute Wert für $t_{\text {ind }}$ kann nicht mit einer einfachen Inhibierungsreaktion erklärt werden, in der jedes aus dem Initiator gebildete Radikal von einem Inhibierungsmolekül eingefangen wird ${ }^{2}$.

Wie Abbildung 6.3 zeigt, ist die experimentell bestimmte Induktionszeit viel kleiner als die Zeit, die nach einem solchen klassischen Inhibierungsmodell berechnet

\footnotetext{
${ }^{2}$ Den technischen Monomeren (z. B. MMA) setzt man häufig Inhibitoren zu, damit vorzeitige Polymerisationen unterbleiben. Inhibitoren werden technisch vor der Polymerisation meist nicht entfernt. Ihre Wirkung wird vielmehr durch gezielte Überdosierung von Initiatoren aufgehoben.
} 


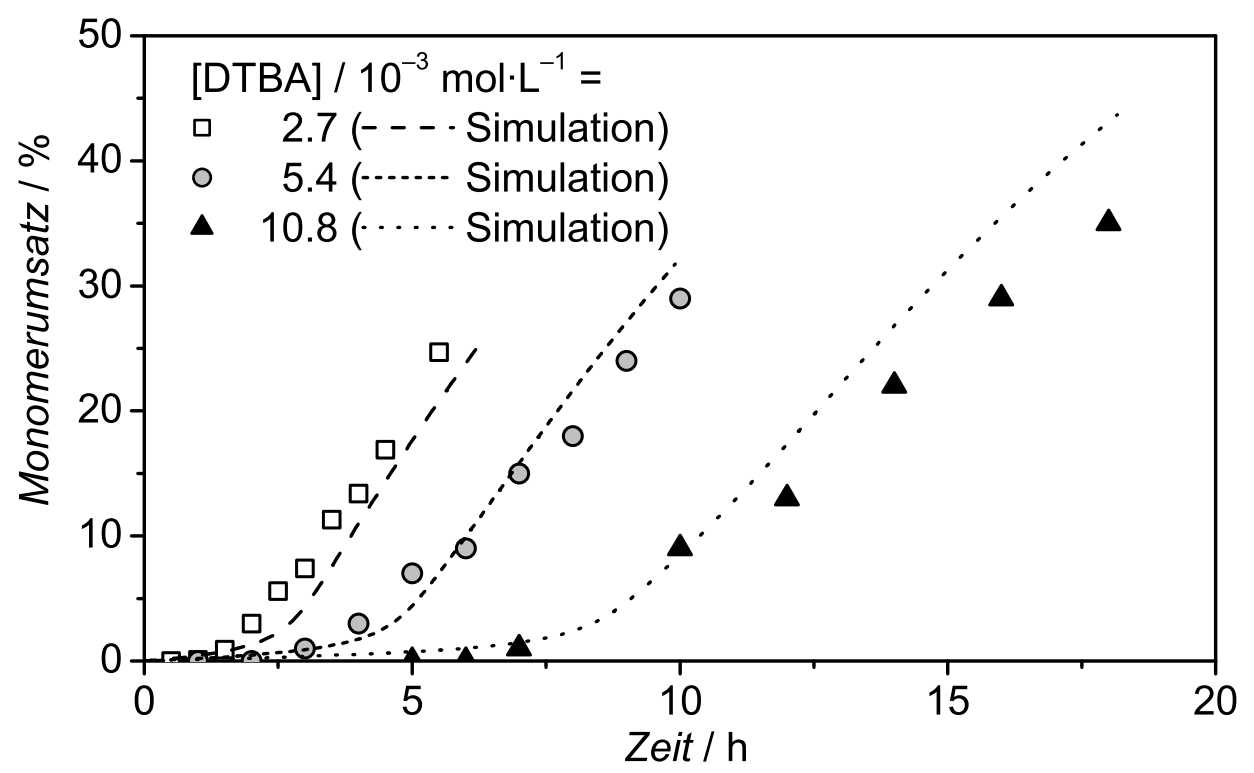

Abbildung 6.2: Monomerumsatz in Abhängigkeit von der Reaktionszeit für die MMAPolymerisation bei $60{ }^{\circ} \mathrm{C}$ mit verschiedenen DTBA-Konzentrationen und $4.9 \cdot 10^{-3} \mathrm{~mol} \cdot \mathrm{L}^{-1}$ AIBN als Initiator. Die unterbrochenen Linien stellen die Werte aus der Simulation dar.

wird. Diese Situation ist offensichtlich anhand der endlichen Induktionsphase von 7.7 Stunden für die DTBA-Konzentration von $10.8 \cdot 10^{-3} \mathrm{~mol} \cdot \mathrm{L}^{-1}$ und die AIBNKonzentration von $4.9 \cdot 10^{-3} \mathrm{~mol} \cdot \mathrm{L}^{-1}$. Für diesen Fall ist die Anzahl von DTBAMoleküle größer als die mögliche totale Anzahl der primären Radikale aus AIBN. Als Folge würde eine unendlich lange Induktionszeit erwartet, wenn DTBA als ein klassischer Inhibitor fungieren würde. Diese Erkenntnis lässt vermuten, dass ein einzelnes Radikal fähig ist, mehr als ein DTBA-Molekül zu verbrauchen. Dies ist ein wichtiger Befund für die Aufklärung des Mechanismus in der Induktionsphase.

\begin{tabular}{ccc}
\hline$[\mathrm{DTBA}] / 10^{-3} \mathrm{~mol} \cdot \mathrm{L}^{-1}$ & $t_{\text {ind }} / \mathrm{h}$ & $R_{\mathrm{P}} / \mathrm{mol} \cdot \mathrm{L}^{-1} \cdot \mathrm{h}^{-1}$ \\
\hline 2.7 & 1.7 & 0.57 \\
5.4 & 3.5 & 0.40 \\
10.8 & 7.7 & 0.32 \\
\hline
\end{tabular}

Tabelle 6.1: Induktionszeit $t_{\text {ind }}$ und Polymerisationsgeschwindigkeit $R_{\mathrm{P}}$ in Abhängigkeit von der DTBA-Konzentration. 


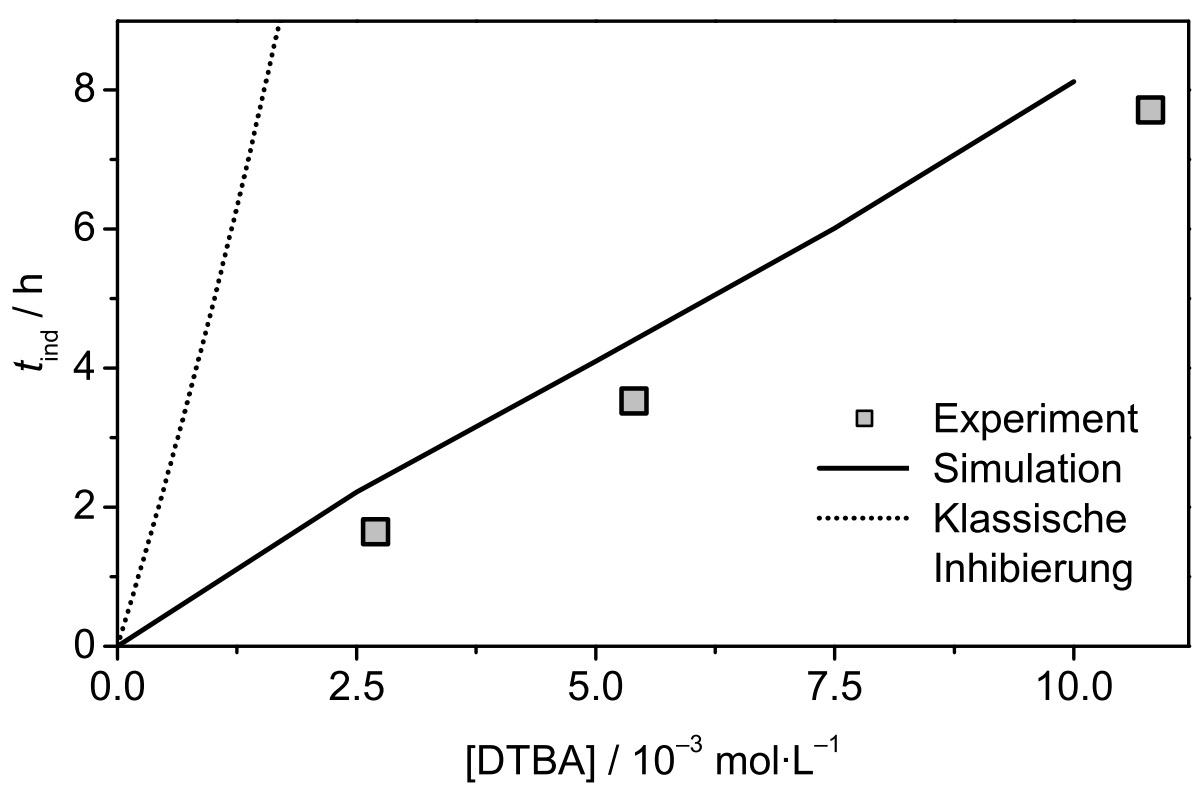

Abbildung 6.3: Induktionszeit $t_{\text {ind }}$ in Abhängigkeit von der DTBA-Konzentration für die Polymerisation von Methylmethacrylat bei $60{ }^{\circ} \mathrm{C}$. Die gestrichelte Linie zeigt die Induktionszeit, die nach der klassischen Inhibierung berechnet wurde.

Nachdem die Induktionszeit vorüber ist, zeigt die Polymerisationsgeschwindigkeit eine leichte Retardierung [125]. Sie ist beim Vergleich zu der Geschwindigkeit einer konventionellen radikalischen Polymerisation kleiner; und zwar nimmt sie bei einer steigenden Kontrollagens-Konzentration ab.

Die Polymerisationsgeschwindigkeit $R_{\mathrm{p}}$ berechnet sich aus der MonomerumsatzReaktionszet-Kurve wie folgt:

$$
R_{\mathrm{p}}=-\frac{\mathrm{d}[\mathrm{M}]}{\mathrm{d} t}=[\mathrm{M}]_{0} \cdot \frac{\mathrm{d} U_{\mathrm{M}}}{\mathrm{d} t}
$$

Dabei stellt $[\mathrm{M}]_{0}$ die Ausgangskonzentration des Monomers da, während $\frac{\mathrm{d} U_{\mathrm{M}}}{\mathrm{d} t}$ für die Steigung der aus der Monomerumsatz-Reaktionszeit-Auftragung erhaltenen Gerade steht. Die Polymerisationsgeschwindigkeit wird näherungsweise über den ausgewählten Umsatzbereich als konstant betrachtet. Effekte wie eine Verringerung der Monomer- und Initiatorkonzentration oder eine erhöhte Viskosität beim hohen Monomerumsatz werden vernachlässigt. 
Die Tabelle 6.1 zeigt, dass mit steigender DTBA-Konzentration die Polymerisationsgeschwindigkeit abnimmt. Bei Vergleich der drei Messreihen ist zu beachten, dass die Initiator-Startkonzentrationen für alle drei Messreihen zwar gleich sind, aber sich durch die unterschiedlich lange Induktionszeit die Initiatorkonzentration beim unterschiedlichen Beginn der Polymerisation geringfügig unterscheiden.

Die beobachtete Retardierung stimmt mit der Annahme überein, dass die Polymerisation mit DTBA im Wesentlichen eine RAFT-Polymerisation darstellt, weil die RAFT-Polymerisation von Methylmethacrylat mit Dithiobenzoaten wie beispielsweise Cumyldithiobenzoat auch eine Retardierung zeigt [126]. Bisher konnte noch nicht endgültig geklärt werden, was genau für Retardierung verantwortlich ist. Sicher scheint nur, dass das im RAFT-Gleichgewicht gebildete intermediäre RAFT-Radikal zu der Retardierung beiträgt. Eine detaillierte Studie dieser Effekt ist eine in dieser Arbeit nicht tiefer behandelte Frage und wurde in der Literatur ausführlich diskutiert [125].

\subsubsection{Kontrollierte radikalische Polymerisation}

Abbildung 6.4 zeigt den Verlauf der mittleren Molmasse des entstehenden Polymers mit dem Monomerumsatz. Man erkennt, dass die Molmasse stetig mit dem Umsatz zunimmt. Außerdem hängt die Molmasse des erhaltenen Polymers von der Anfangskonzentration des eingesetzten DTBA indirekt proportional ab. Die Polydispersität ist näherungsweise im Bereich von 1.2 bis 1.4. All diese Beobachtungen weisen darauf hin, dass dieser Prozess eine kontrollierte radikalische Polymerisation ist und dass DTBA ein effizientes kontrollierendes Agens für die Polymerisation von Methylmethacrylat ist.

Die Krümmungen in der Molmasse-Umsatz-Auftragung, welche besonders bei relativ kleiner DTBA-Konzentration auftritt, stimmen mit der theoretischen Berechnung für die Molmasse des Polymers überein, welches in einer kontrollierten Polymerisation mit kontinuierlicher Initiierung gebildet wird. Die theoretische Berechnung ist in Gleichung 6.2 beschrieben, in welcher die permanente Zunahme der Anzahl der Polymerketten in der Nenner berücksichtigt ist [33].

$$
M_{\mathrm{n}}^{\text {theo }}=\frac{[\mathrm{M}]_{0} \cdot M W_{\text {Monomer }} \cdot U_{\mathrm{M}}}{[\mathrm{CA}]_{0}+d \cdot \frac{1}{2} \cdot \int_{0}^{t} R_{i} \mathrm{~d} t}=\frac{[\mathrm{M}]_{0} \cdot M W_{\text {Monomer }} \cdot U_{\mathrm{M}}}{[\mathrm{CA}]_{0}+d \cdot f \cdot\left([\mathrm{I}]_{0}-[\mathrm{I}]_{U}\right)}
$$




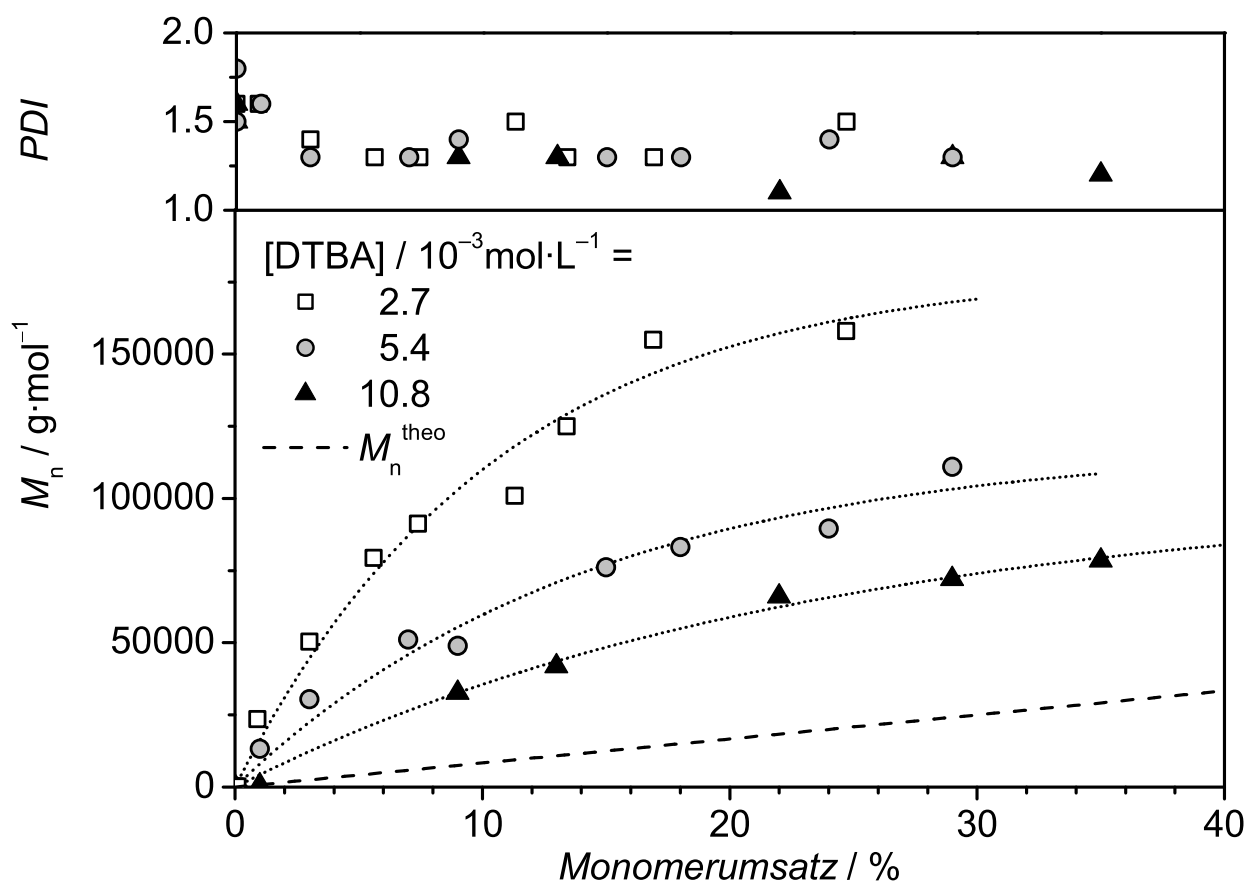

Abbildung 6.4: Auftragung des Zahlenmittels der Molmassen $M_{\mathrm{n}}$ und Polydispersität PDI gegen den Monomerumsatz für die Polymerisation von MMA bei $60{ }^{\circ} \mathrm{C}$ mit verschiedenen DTBA-Konzentrationen und $4.9 \cdot 10^{-3} \mathrm{~mol} \cdot \mathrm{L}^{-1}$ AIBN. Die gestrichene Linie stellt die theoretischen Molmasse für die Messreihe $[\mathrm{DTBA}]_{0}=10.8 \cdot 10^{-3} \mathrm{~mol} \cdot \mathrm{L}^{-1}$ dar. Aus Gründen der Übersichtlichkeit wurde nur diese von den drei Verläufen der theoretischen Molmasse aufgetragen. Die gepunkteten Linien sind Leitlinien.

In dieser Gleichung ist $M_{\mathrm{n}}^{\text {theo }}$ die theoretische Molmasse, $M W_{\text {Monomer }}$ die Molmasse vom Monomer, $[\mathrm{CA}]_{0}$ die Startkonzentration vom kontrollierenden Agens, $d$ hängt von Terminierungsmodus $\mathrm{ab}^{3}, R_{i}$ ist die Zerfallsgeschwindigkeit des Initiators, $f$ die Initiatoreffektivität, $[\mathrm{I}]_{0}$ die Startkonzentration vom Initiator und $[\mathrm{I}]_{U}$ die Initiatorkonzentration beim Monomerumsatz $U$. Die für die Berechnung benötigten Parameter sind in der Tabelle 6.5 angegeben.

Bei der Berechnung der theoretischen Molmasse wird angenommen, dass DTBA direkt als kontrollierendes Agens in der Polymerisation agiert, d. h. $[\mathrm{CA}]_{0}=[\mathrm{DTBA}]_{0}$.

\footnotetext{
${ }^{3} d$ ist die Anzahl der Polymerkette, die beim Terminierungsprozess gebildet wird: $d=1$ für Terminierung über Kombination und $d=2$ für Terminierung über Disproportionierung.
} 
Die theoretische Molmasse $M_{\mathrm{n}}^{\text {theo }}$ ist deutlich kleiner als die experimentellen Werte. Diesen Effekt kann man in Abbildung 6.4 erkennen, in der die theoretischen Molmassen für $[\mathrm{DTBA}]_{0}=10.8 \cdot 10^{-3} \mathrm{~mol} \cdot \mathrm{L}^{-1}$ zur direkten Vergleich mit den experimentellen Werten eingezeichnet sind. Die gleiche Beobachtung über die theoretische Molmasse wurde in der Literatur für die Polymerisation von Styrol und Methylacrylat mit Phenyldithioessigsäure erwähnt [121]. Dass die theoretische Molmasse viel höher ist als die experimentellen Werte, weist auf die schlechte Effektivität der Dithiobenzoesäure hin, d. h. nicht alle DTBA-Moleküle werden effektiv in ein kontrollierendes Agens umgewandelt.

\subsubsection{Effektive RAFT-Agens-Konzentration}

Die Konzentration an kontrollierenden Agens, das effektiv die Polymerisation von MMA kontrolliert, ist niedriger als die eingesetzte Dithiobenzoesäure. Um die Konzentration vom effektiven kontrollierenden Agens $[\text { RAFT }]_{\text {eff }}$ systematisch und quantitativ zu bestimmen, wird die Gleichung 6.2 nach $[\mathrm{CA}]_{0}$ umgeformt $^{4}$. Durch Ersetzen von $[\mathrm{CA}]_{0}$ mit $[\mathrm{RAFT}]_{\text {eff }}$ und $[\mathrm{I}]_{0}$ mit $[\mathrm{I}]_{\text {ind }}$ entsteht die Gleichung 6.3 .

$$
[\mathrm{RAFT}]_{\mathrm{eff}}=\frac{[\mathrm{M}]_{0} \cdot M W_{\mathrm{Monomer}} \cdot U_{\mathrm{M}}}{M_{\mathrm{n}}}-d \cdot f \cdot\left([\mathrm{I}]_{\mathrm{ind}}-[\mathrm{I}]_{U}\right)
$$

Diese Gleichung berücksichtigt den Umstand, dass die eigentliche Polymerisation erst nach der Induktionszeit startet, wenn die eigentliche Initiator-Konzentration $[\mathrm{I}]_{\text {ind }}$ beträgt. Die vom Initiator stammenden Radikale, die während der Induktionsphase gebildet werden, werden in Gleichung 6.3 nicht berücksichtigt, weil das in dieser Phase entstehende Material kein Polymer ist.

Die effektive RAFT-Agens-Konzentration $[\text { RAFT }]_{\text {eff }}$ wird aus den experimentellen Daten der Molmasse mit Hilfe der Gleichung 6.3 berechnet. In Abbildung 6.5 wird die effektive RAFT-Agens-Konzentration in der MMA-Polymerisation gegen die Gesamtmenge der Radikale, die aus dem Initiatorzerfall in das System abgegeben wurden, aufgetragen. Drei wichtige Aussagen können aufgrund dieser Abbildung gemacht werden:

\footnotetext{
${ }^{4}[\mathrm{RAFT}]_{\mathrm{eff}}$ ist mit der Konzentration des lebenden Polymers bzw. des Makro-RAFT-Agens gleichzusetzen.
} 


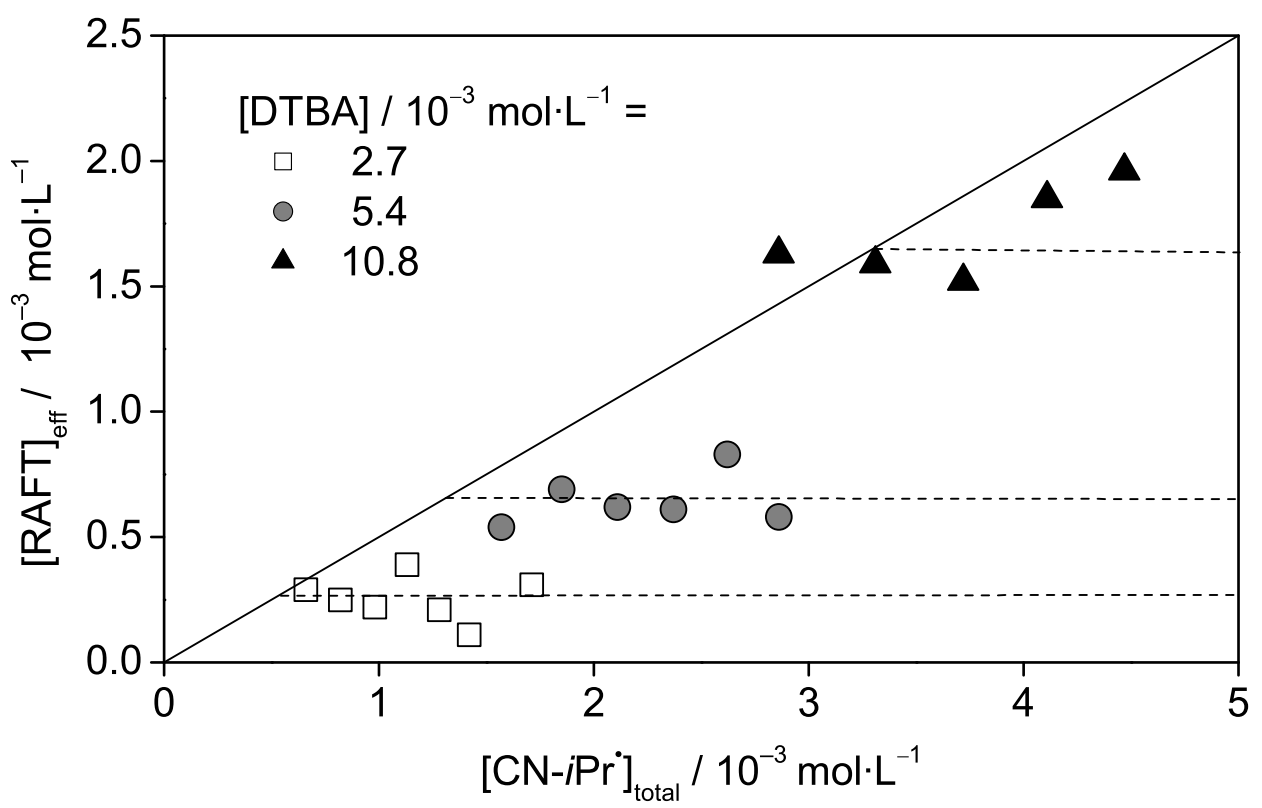

Abbildung 6.5: Effektive RAFT-Agens-Konzentration [RAFT $]_{\text {eff }}$ in Abhängigkeit von der Gesamtmenge der Cyanoisopropylradikale $[\mathrm{CN}-i \operatorname{Pr} \bullet$, die aus dem AIBN-Zerfall in die MMA-Polymerisation bei $60{ }^{\circ} \mathrm{C}$ mit verschiedenen DTBA-Konzentrationen abgegeben wurden. Die Parameter für die Berechnung sind in Tabelle 6.5 aufgelistet.

1. Nachdem die Induktionszeit vorbei ist, bleibt die effektive RAFT-Agens-Konzentration näherungsweise konstant. Die horizontalen gestrichelten Linien in der Abbildung 6.5 versuchen, dies zu veranschaulichen. Weitere Zufuhr von Radikalen führt nicht zu einer Erhöhung der RAFT-Agens-Konzentration. Da die Abszisse auch die Reaktionszeit widerspiegelt, ist es offensichtlich, dass die Bildung des RAFT-Agens während der Polymerisationsphase nicht mehr stattfindet.

2. Da die Polymerisation in der Induktionsphase noch nicht startet, gibt es in diese Phase keine Daten der Molmasse. Deshalb ist es nicht möglich die $\mathrm{Zu}$ nahme der effektiven RAFT-Agens-Konzentration während der Induktionszeit mit dieser Methode zu bestimmen. Eine detaillierte Analyse der Abbildung 6.5, die eine lineare Funktion mit einer Steigerung von 0.5 beinhaltet, lässt vermuten, dass zwei Radikale nötig sind, um ein effizientes RAFT-Agens zu bilden. 
Diese Beobachtung weist darauf hin, dass eine Terminierungsreaktion von zwei Radikalen für die Bildung eines RAFT-Agens verantwortlich ist und dass dieser Prozess nur in der Induktionsphase stattfindet.

3. Die effektive RAFT-Agens-Konzentration ist deutlich kleiner als die eingesetzte DTBA-Konzentration. Z. B. für den Fall $[\mathrm{DTBA}]=10.8 \cdot 10^{-3} \mathrm{~mol} \cdot \mathrm{L}^{-1}$ wird eine effektive RAFT-Agens-Konzentration von $1.6 \cdot 10^{-3} \mathrm{~mol} \cdot \mathrm{L}^{-1}$ bestimmt. Nur rund $15 \%$ der Dithiobenzoesäure wird in das effiziente RAFT-Agens umgewandelt. Für die anderen DTBA-Konzentrationen werden ähnliche Werte gefunden.

Die effektive RAFT-Agens-Konzentration ist nicht nur abhängig von der eingesetzten DTBA-Konzentration, sie ist also auch abhängig von der Gesamtmenge der vom Initiator stammenden Radikale. Um die Ausbeute von RAFT-Agens zu erhöhen, kann man die Radikal- bzw. die Initiator-Konzentration erhöhen.

\subsubsection{Endgruppenanalyse}

Die Annahme, dass die Polymerisation mit Dithiobenzoesäure im Wesentlichen eine RAFT-Polymerisation mit einem Dithiobenzoat darstellt, wurde von der Forschungsgruppe um Pan durch die Endgruppenanalyse mit Hilfe der NMR-Spektroskopie gestärkt [84]. Die Dithiobenzoat-Gruppe, die charakteristisch für Makro-RAFTAgens aus einer lebenden Polymerisation ist, wurde dadurch identifiziert. Dieser Befund wurde durch die Gruppe um Monteiro unterstützt, die eine für DithiocarbonylVerbindung typische UV-Absorption des Polymers aus der Polymerisation mit der Dithiosäure beobachtete [121].

Die Analyse der Endgruppen und damit die Bestimmung der Funktionalisierung vom gebildeten Polymer ist durch die geringe Konzentration der Endgruppen verglichen mit der Wiederholungseinheit schwierig. Daher ist diese Analyse besonders gut geeignet für Polymere mit niedrigen Polymerisationsgraden. Zur Bestimmung der Endgruppen des entstehenden Polymers werden in dieser Arbeit UV-Spektroskopie und ESI-Massenspektrometrie eingesetzt. Im Folgenden werden die Ergebnisse dieser zwei Analysenmethoden im Detail diskutiert. 
Endgruppenanalyse mit der UV-Spektroskopie

Der Nachweis einer Dithiobenzoat-Gruppe und deren Anzahl pro Polymerkette sind in Abbildung 6.6 illustriert. Zuerst wird die Molmassenverteilung des Polymers mittels GPC-Experiments mittels eines Brechungsindex-Detektors (RI-Detektor ${ }^{5}$ ) gemessen. Bei dem RI-Detektor ist die Intensität des Signals proportional zur MassenKonzentration des Polymers in der mobilen Phase. Das RI-Signal gibt deshalb die sogenannte Massenverteilung gegen der Molmasse in logarithmischer Skala wieder. Unter Verwendung zweier in Reihe geschalteter Detektoren wird gleichzeitig die Molmassenverteilung mit einem UV-Absorptions-Detektor analysiert. Der UV-Detektor detektiert bei einer Wellenlänge von $\lambda=254 \mathrm{~nm}$ ausschließlich die Thiolcarbonylthio-Gruppe, die wegen des Elektronenüberganges $\pi \rightarrow \pi^{*}$ Energie dieser Wellenlänge stark absorbiert [127]. Die Polymethylmethacrylat-Kette ist bei $\lambda=254 \mathrm{~nm}$ weitgehend unsichtbar [128]. Deshalb ist das UV-Absorptionssignal proportional zur Anzahl der Endgruppen des Polymers.

Die aus dem UV-Detektor erhaltene Zahlenverteilung wird folglich in eine Massenverteilung mathematisch nach Gleichung 6.4 umgewandelt. Dabei wird angenommen, dass jede Polymerkette eine absorbierende Endgruppe trägt.

$$
I_{\mathrm{RI}}=f \cdot I_{\mathrm{UV}} \cdot M_{\mathrm{P}}=w_{\log M}
$$

dabei stellt $I_{\mathrm{RI}}$ die RI-Detektorintensität, $f$ den angepassten Proportionalitätsfaktor, $I_{\mathrm{UV}}$ die UV-Detektorintensität, $M_{\mathrm{P}}$ die Molmasse des Polymers und $w_{\log M}$ die Massenverteilung gegen eine logarithmische Molmassenachse dar.

In Abbildung 6.6 sind das RI-Signal und das umgewandelte UV-Signal aufgetragen. Die Betrachtung der beiden Kurven ergibt eine gute Übereinstimmung der beiden Molmassenverteilung. Dieser Befund beweist eine stark absorbierende Gruppe im Polymer, das in der Anwesenheit von der Dithiobenzoesäure gebildet wird. Es bestätigt außerdem, dass jede Polymerkette eine absorbierende Gruppe trägt. Weiter weist es auf eine typische RAFT-Polymerisation mit dem Dithiobenzoat hin.

Die Molmassenverteilung in Abbildung 6.6 stammt vom Polymer, das kurz nach der Induktionsphase bei einem kleinen Monomerumsatz von $2.9 \%$ und in einem System mit einer relativ hohen DTBA-Konzentration gebildet wurde. Diese Reaktionsbedingungen führen zu einer relativ kleinen mittleren Molmasse, welche das

\footnotetext{
${ }^{5}$ Englisch: refractive index
} 


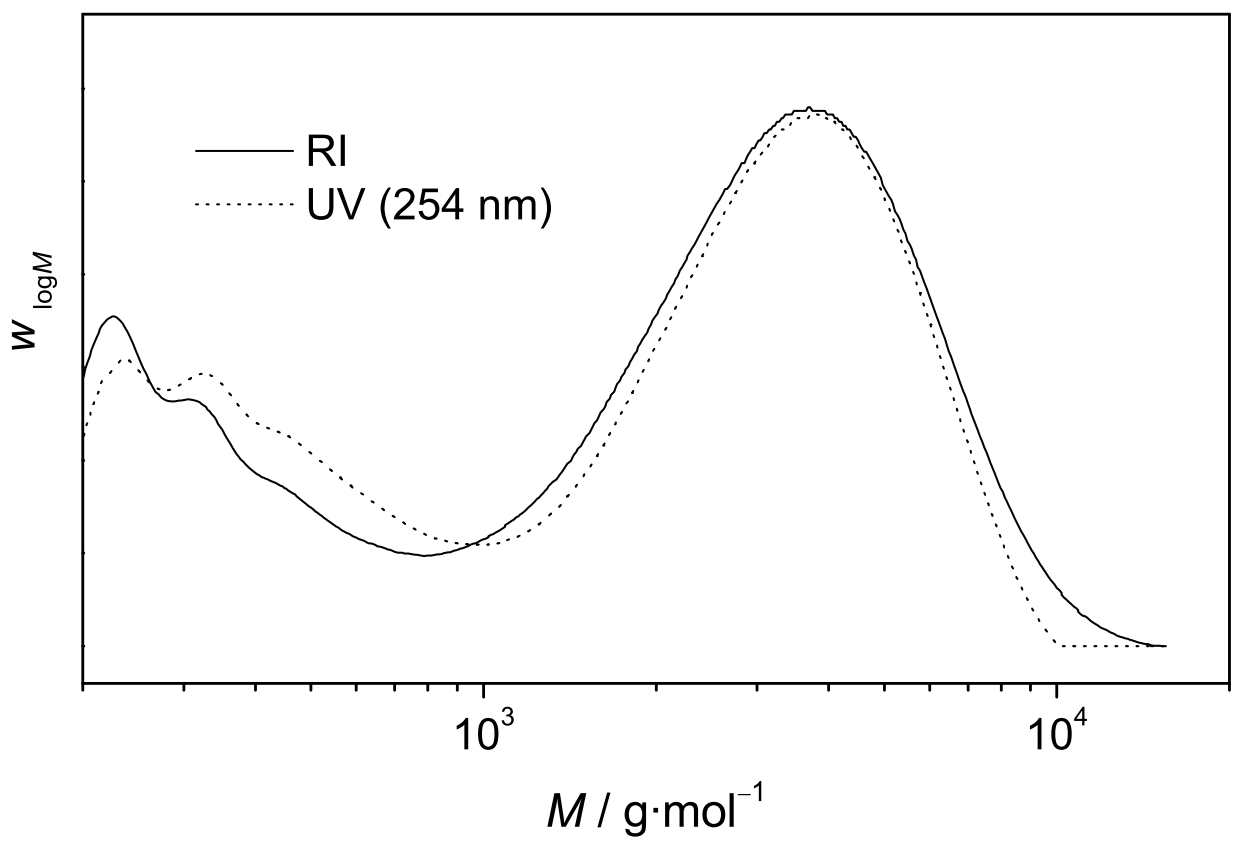

Abbildung 6.6: Molmassenverteilungen gegen die Molmasse in logarithmischer Skala, die aus dem RI-Signal und dem umgewandelten UV-Signal $(\lambda=254 \mathrm{~nm})$ stammen. Das Polymer wird aus der Reaktion von MMA mit DTBA $\left(51 \cdot 10^{-3} \mathrm{~mol} \cdot \mathrm{L}^{-1}\right)$ und AIBN $\left(5.0 \cdot 10^{-3} \mathrm{~mol} \cdot \mathrm{L}^{-1}\right)$ bei $100{ }^{\circ} \mathrm{C}$ gebildet. Der Monomerumsatz beträgt $2.9 \%$ bei einer Reaktionszeit von 4.5 Stunden. Das UV-Signal wurde in die GPC-Verteilung $w_{\log M}$ entsprechend Gleichung 6.4 umgewandelt.

Signal-Rausch-Verhältnis in der Endgruppenanalyse erhöht. Mit steigendem Monomerumsatz bzw. mit höheren Molmassen des Polymers wird die Verteilung enger, und durch die erhöhte Menge des Polymermaterials wird das niedermolekulare Material aus Abbildung 6.6 zunehmend unbedeutender.

\section{Endgruppenanalyse mit der ESI-Massenspektrometrie}

Elektrospray-Ionisation-Massenspektrometrie (ESI-MS) erlaubt eine sehr detaillierte Endgruppenanalyse des Polymers [129, 130, 131]. Diese Massenspektrometrietechnik ist besonders schonend im Hinblick auf den Inonisierungsprozess, sodass die relativ schwach gebundene Thiocarbonylthio-Gruppe während der Analyse komplett erhalten bleibt [120, 132]. 


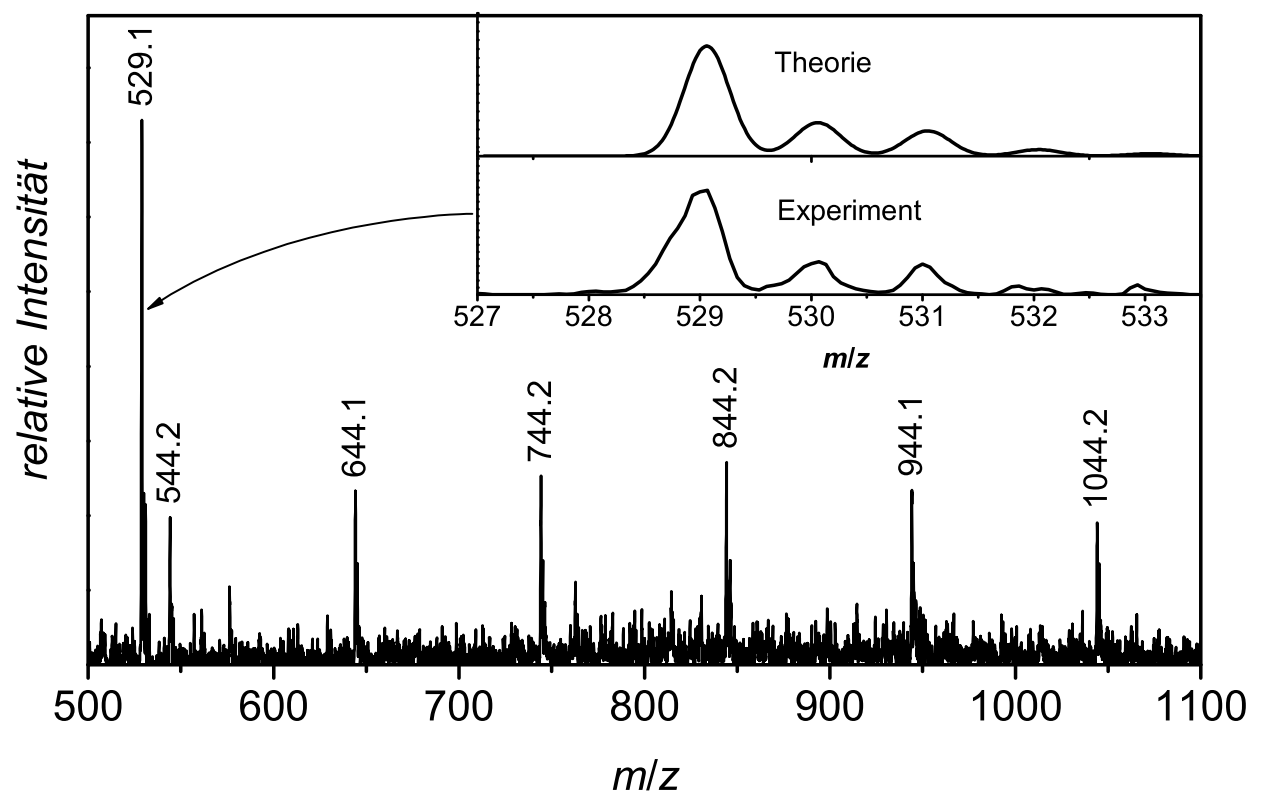

Abbildung 6.7: ESI-MS-Spektrum vom $\mathrm{Na}^{+}$-ionisierten Polymethylmethacrylat, das aus der Polymerisation mit DTBA $\left(10.8 \cdot 10^{-3} \mathrm{~mol} \cdot \mathrm{L}^{-1}\right)$ und AIBN $\left(4.9 \cdot 10^{-3} \mathrm{~mol} \cdot \mathrm{L}^{-1}\right)$ bei $60{ }^{\circ} \mathrm{C}$ gebildet wird. Der Monomerumsatz beträgt nach fünf Stunden Reaktionszeit $0.5 \%$. Die kleinen Diagramme stellen die Vergrößerung des Signals bei $m / z=529.1$ und die theoretische Isotopenverteilung für die Spezies $\mathbf{1 1}\left(\mathrm{C}_{24} \mathrm{H}_{26} \mathrm{O}_{4} \mathrm{~S}_{4}+\mathrm{Na}^{+}\right)$dar.

Die Abbildung 6.7 zeigt einen Ausschnitt des Massenspektrums vom Polymethylmethacrylat, das in einer Polymerisation mit DTBA bei $60{ }^{\circ} \mathrm{C}$ synthetisiert wurde. Das Spektrum stellt die Situation am Ende der Induktionsphase dar. Eine Serie von wiederholenden Signalen, die einen Abstand von ca. $100.1 \mathrm{amu}$ d. h. von der Molmasse einer MMA-Monomereinheit haben, ist deutlich sichtbar. Die $m / z$-Werte der Signalserie weisen auf MMA-Oligomere hin, die als Endgruppen eine Cyanopropylsowie eine Dithiobenzoat-Gruppe tragen (siehe Spezies 13 in Abbildung 6.10).

Bemerkenswert ist das starke Einzelsignal bei $m / z=529.1$, das möglicherweise zu der Spezies 11 in Abbildung 6.10 und Tabelle 6.3 gehört. Die Spezies 11 hat zwei Dithiobenzoat-Gruppe und zwei Monomereinheiten. Wahrscheinlich stammt sie aus der Dimerisationsreaktion zwei gleicher Teilchen. Beim Vergleich der experimentellen Isotopenstruktur des Signals bei $m / z 529.1$ mit der berechneten Isotopenvertei- 
lung für die molekulare Zusammensetzung $\mathrm{C}_{24} \mathrm{H}_{26} \mathrm{O}_{4} \mathrm{~S}_{4}+\mathrm{Na}^{+}$zeigt sich eine große Übereinstimmung, welche auf die richtige Interpretation dieses Signals hinweist.

Um andere in niedriger Konzentration vorliegende Spezies zu identifizieren, werden die Reaktionsbedingungen der MMA-Polymerisation mit DTBA verändert. Die Konzentration des primären Radikals aus AIBN wird durch die Steigerung der Polymerisationstemperatur auf $100{ }^{\circ} \mathrm{C}$ erhöht. Außerdem wird eine größere Einwaage von DTBA $\left(51 \cdot 10^{-1} \mathrm{~mol} \cdot \mathrm{L}^{-1}\right)$ verwendet. Abbildung 6.8 zeigt einen Ausschnitt des ESI-MS-Spektrums vom Oligomermaterial, das in der früheren Phase der MMAPolymerisation mit den veränderten Bedingungen entsteht.

Beim Vergleich mit dem Massenspektrum aus Abbildung 6.7 fällt auf, dass zusätzlich zu der Signalserie der Spezies 13 eine andere Signalserie des MMA-Oligomers aufgetreten ist. Die $m / z$-Werte dieser Signalserie weisen auf MMA-Oligomere hin, die als Endgruppen eine Cyanopropylgruppe und einen Wasserstoff tragen (siehe Spezies 6 in Abbildung 6.10). Da diese MMA-Oligomere keine Dithiobenzoat-Gruppe enthalten, sind sie offensichtlich keine lebenden, sondern toten Polymerketten. Das tote Oligomermaterial kann denkbarerweise durch folgende zwei Reaktionswege gebildet werden 6 .

1. Ein primäres Radikal addiert an ein Monomer und wird in ein propagierendes Oligomerradikal umgewandelt. Das Oligomerradikal abstrahiert anschließend einen am Schwefelatom gebundenen Wasserstoff von DTBA. Diese Reaktion ergibt ein MMA-Oligomer mit einer Cyanoisopropyl-Gruppe und einem Wasserstoff als Endgruppen.

2. Das Oligomerradikal geht mit einem anderen Radikal eine Terminierungsreaktion ein. Es entsteht dabei nach dem Disproportionierungsmechanismus ein MMA-Oligomer mit einer Cyanoisopropyl-Gruppe und einem Wasserstoff als Endgruppen. Dieser zweite Weg über die Terminierung kann aus zwei Gründen ausgeschlossen werden.

- Zum einen findet man kein Produkt aus der Terminierung des Oligomerradikals nach dem Kombinationsmechanimus, obwohl zwei MMA-Radikale

\footnotetext{
${ }^{6}$ Die Transferreaktion zum Polymer oder Monomer, welche auch zu einem Polymer mit einem Wasserstoff als Endgruppe führt, kann wegen der relativ langsamen Geschwindigkeit der Transferreaktion vernachlässigt werden.
} 


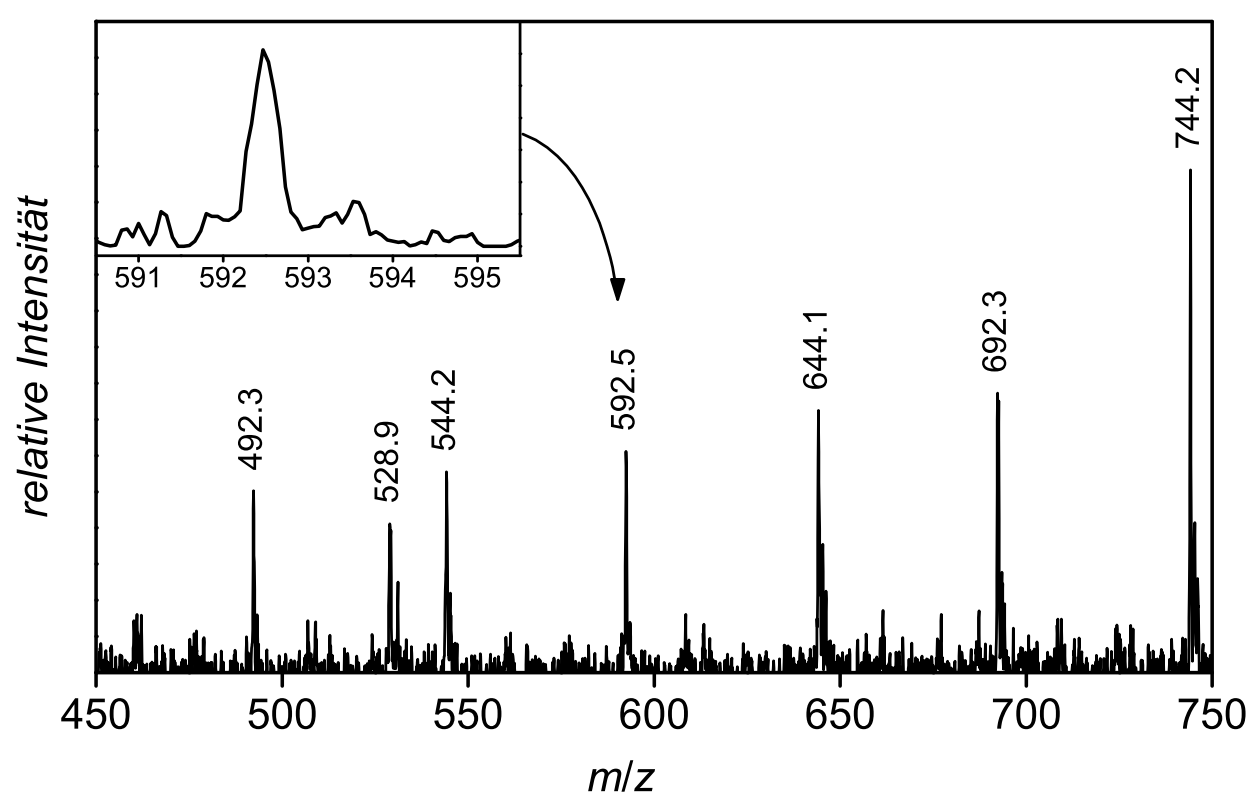

Abbildung 6.8: ESI-MS-Spektrum vom $\mathrm{Na}^{+}$-ionisierten Polymethylmethacrylat, das aus der Polymerisation mit DTBA $\left(51 \cdot 10^{-3} \mathrm{~mol} \cdot \mathrm{L}^{-1}\right)$ und AIBN $\left(5.0 \cdot 10^{-3} \mathrm{~mol} \cdot \mathrm{L}^{-1}\right)$ bei $100{ }^{\circ} \mathrm{C}$ gebildet wird. Der Monomerumsatz beträgt nach einer halbe Stunden Reaktionszeit $1.2 \%$. Das kleine Diagramm stellt die Vergrößerung des Signals bei $m / z 592.5$ dar.

durchaus miteinander kombinieren [133].

- Zum anderen ist es die Signalsstruktur, die im kleinen Diagramm in der Abbildung 6.8 vergrößert dargestellt ist. Sie macht deutlich, dass dieses Signal nicht vom Terminierungsprodukt zweier Oligomerradikale über die Disproportionierung stammt, denn solch eine Disproportionierungsreaktion ergibt durch die Wanderung von Wasserstoff gleichzeitig ein zweites Oligomer, das durch zwei Masseneinheiten vom ersten getrennt ist. Dadurch entsteht eine typische Zwilling-Signalstruktur in dem ESI-MSSpektrum. Die Abwesenheit dieses zweiten Signals weist darauf hin, dass das tote Oligomer nicht durch Disproportionierung bzw. Terminierung entstanden ist.

Es kann nun ohne Zweifel behauptet werden, dass das tote Polymer durch die Abstrahierung eines Wasserstoffs gebildet wurde. Wahrscheinlich stammt dieser Was- 
serstoff aus der Dithiobenzoesäure, die in diesem Fall als Transfer-Agens fungiert.

Ausgehend von dieser Annahme, soll nun nach der Antwort gesucht werden, warum das tote Polymer bei der MMA-Polymerisation bei $60{ }^{\circ} \mathrm{C}$ nicht auftritt. Da das tote Oligomer durch die Transferreaktion zu DTBA entsteht, soll die Temperaturabhängigkeit der Transferreaktion betrachtet werden. Leider gibt es in der Literatur noch keine Daten über die Transferreaktion von Dithiobenzoesäure. Jedoch kann, um Rückschlüsse zu gewinnen, die Transferkonstante einer ähnliche TransferagensFamilie, z. B. von einem Alkylthiol, herangezogen werden.

In Tabelle 6.2 sind die aus der Literatur entnommenen Transferkonstanten für den Temperaturbereich von $60-100{ }^{\circ} \mathrm{C}$ für MMA-Polymerisation mit 1-Dodecanethiol als Transferagens aufgelistet [134]. Man erkennt deutlich, dass die Transferkonstante mit steigender Temperatur abnimmt. Der Transferkonstante bei $60{ }^{\circ} \mathrm{C}$ ist fast dreimal so groß wie bei $100{ }^{\circ} \mathrm{C}$.

\begin{tabular}{cc}
\hline Temperatur & Transferkonstant \\
\hline $60{ }^{\circ} \mathrm{C}$ & 0.70 \\
$70{ }^{\circ} \mathrm{C}$ & 0.55 \\
$80{ }^{\circ} \mathrm{C}$ & 0.42 \\
$90{ }^{\circ} \mathrm{C}$ & 0.33 \\
$100{ }^{\circ} \mathrm{C}$ & 0.26 \\
\hline
\end{tabular}

Tabelle 6.2: Transferkonstante von 1-Dodecanthiol als Transferagens in der Polymerisation von MMA [134].

Wenn man annimmt, dass die Temperaturabhängigkeit der Transferkonstante für DTBA ähnlich ist wie die für 1-Dodecanethiol, kann man die Befunde aus den ESI-MS-Experimenten erklären. Bei der Polymerisationstemperatur von $60{ }^{\circ} \mathrm{C}$ ist die Wasserstoffabstrahierung bzw. die Transferreaktion eines propagierenden Radikals so groß, dass nur die Oligomere mit einer Molmasse von weniger als $500 \mathrm{~g} \cdot \mathrm{mol}^{-1}$ (z. B. mit $m / z$ von 192, 292, $392 \mathrm{~g} \cdot \mathrm{mol}^{-1}$ ) gebildet werden. Diese Oligomere treten dann nicht im ESI-MS-Spektrum auf, weil die ESI-MS-Technik die polymeren Spezies mit einer Molmasse niedriger als $500 \mathrm{~g} \cdot \mathrm{mol}^{-1}$ nicht gut detektiert. Bei der Polymerisationstemperatur von $100{ }^{\circ} \mathrm{C}$ ist die Wasserstoffabstrahierung im Vergleich zu der Propagierung relativ langsam, so dass auch Oligomere mit einer Molmasse von mehr als $500 \mathrm{~g} \cdot \mathrm{mol}^{-1}$ gebildet und mit der ESI-MS-Technik nachgewiesen werden können. 


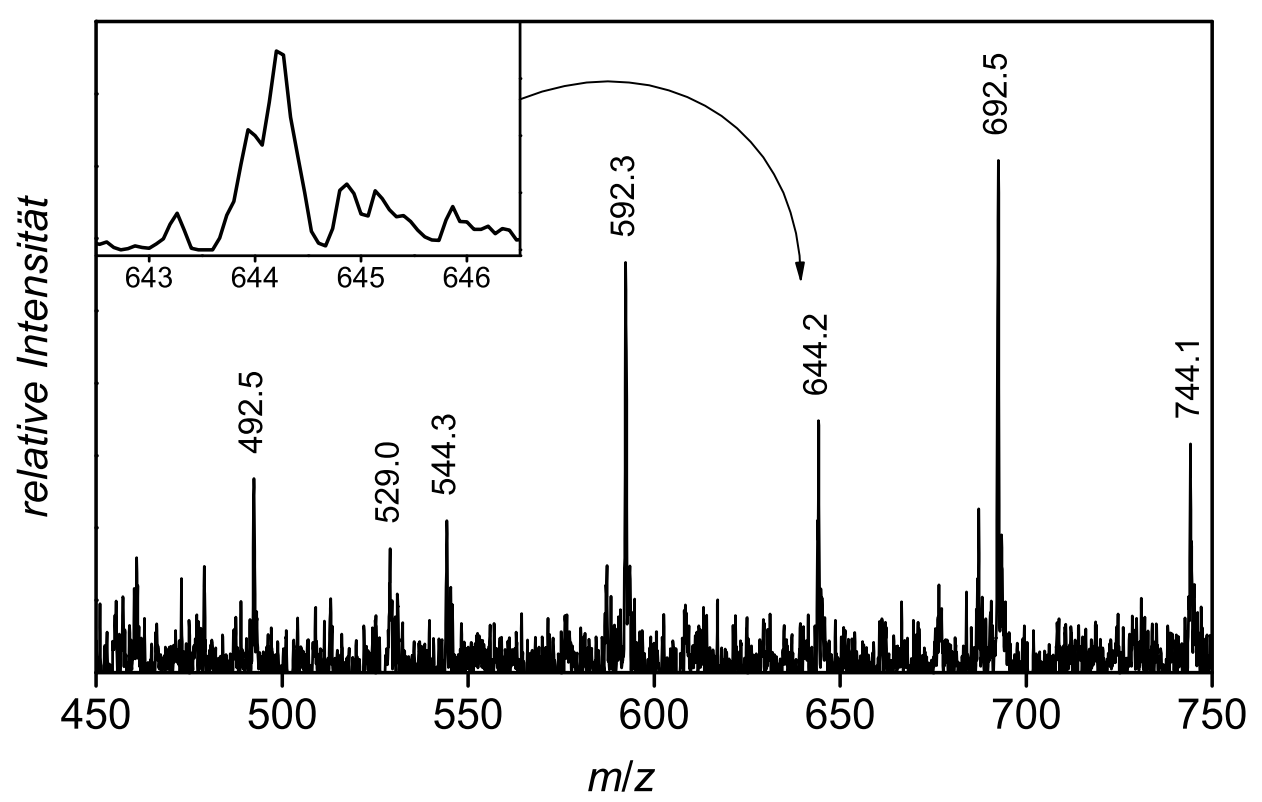

Abbildung 6.9: ESI-MS-Spektrum vom $\mathrm{Na}^{+}$-ionisierten Polymethylmethacrylat, das aus der Polymerisation mit DTBA $\left(51 \cdot 10^{-3} \mathrm{~mol} \cdot \mathrm{L}^{-1}\right)$ und AIBN $\left(5.0 \cdot 10^{-3} \mathrm{~mol} \cdot \mathrm{L}^{-1}\right)$ bei $100{ }^{\circ} \mathrm{C}$ entstanden wird. Der Monomerumsatz beträgt nach 4.5 Stunden Reaktionszeit 2.9\%. Das kleine Diagramm stellt die Vergrößerung des Signals bei $m / z 644.1$ dar.

Ein weiterer Hinweis, dass die MMA-Polymerisation mit DTBA ein lebenden Prozess ist, tritt beim Vergleich der Abbildungen 6.8 und 6.9 auf. Die Polymerisation in Abbildung 6.8 dauerte nur eine halbe Stunde und der Monomerumsatz betrug $1.2 \%$. Die Polymerisation in Abbildung 6.9 dauerte viereinhalb Stunde und der Umsatz betrug 2.9 \% . Das Verhältnis vom Signal des toten Oligomermaterials zu dem Signal des lebenden RAFT-Polymers ist mit der Zeit bzw. mit dem Umsatz größer.

Diese Beobachtung kann wie folgt erklärt werden. Sowohl die Anzahl des toten Oligomermaterials als auch die des lebenden RAFT-Polymers bleiben nach der Induktionszeit weitgehend konstant. Doch während die Molmassenverteilung des toten Oligomermaterials gleich bleibt, verschiebt sich die Molmassenverteilung des lebenden RAFT-Polymers mit dem steigenden Monomerumsatz zu höheren Molmassen. Die Menge an lebendem Polymer im Bereich $450 \mathrm{bis} 750 \mathrm{~g} \cdot \mathrm{mol}^{-1}$ wird wegen des 
Kettenwachstums kleiner. Deshalb wird das Verhältnis von Signal des toten Oligomermaterials zu dem Signal des lebenden Polymers in diesem Abschnitt des ESIMS-Spektrums mit steigendem Umsatz größer.

Die ESI-MS-Endgruppenanalyse liefert uns den Beweis für die Existenz der folgenden drei Spezies: das effektive RAFT-Polymer 13, die auffällig markante Spezies 11 und das tote Oligomermaterial 6. Tabelle 6.3 gibt die Ergebnisse aus den ESIMS-Spektren zusammenfassend wieder.

\begin{tabular}{cccc}
\hline Spezies & Polymerisationsgrad & experimentell $\mathrm{m} / \mathrm{z}$ & theoretisch $\mathrm{m} / \mathrm{z}$ \\
\hline $\mathbf{6}$ & 5 & 592.5 & 592.3 \\
& 6 & 692.3 & 692.4 \\
& 7 & 792.5 & 792.4 \\
\hline $\mathbf{1 3}$ & 3 & 544.2 & 544.2 \\
& 4 & 644.1 & 644.2 \\
& 5 & 744.3 & 744.3 \\
\hline $\mathbf{1 1}$ & - & 529.1 & 529.1 \\
\hline
\end{tabular}

Tabelle 6.3: Experimentelle und theoretische $m / z$-Werte der $\mathrm{Na}^{+}$-ionisierten Spezies, die bei der MMA-Polymerisation mit DTBA entstehen.

\subsubsection{Vorgeschlagener Mechanismus in der Induktionsphase}

Aufgrund der bisherigen experimentellen Hinweise wird ein Reaktionsmechanismus für die Bildung von RAFT-Agens aus Dithiobenzoesäure während der Induktionsphase postuliert. Die Mechanismus wird in Abbildung 6.10 mit AIBN als Initiator und MMA als Monomer illustriert.

Das aus dem Initiator stammende Radikal 4 addiert an ein Monomer und wird in ein propagierendes Oligomerradikal 5 umgewandelt. Das Oligomerradikal abstrahiert dann ein am Schwefelatom gebundenes Wasserstoffatom. Diese Reaktion ergibt ein MMA-Polymer 6 mit einer Cyanoisopropyl-Gruppe und einem Wasserstoffatom als die Endgruppen. Außerdem entsteht ein Phenylcarbothioylsulfanyl-Radikal 7. Natürlich kann die Abstrahierungsreaktion zu einem geringen Anteil auch mit einem Primärradikal 4 erfolgen. Wegen der Übersichtlichkeit wird diese Reaktion nicht im mechanistischen Schema gezeigt.

Wegen folgender Gründe verläuft die Wasserstoffabstrahierung von DTBA mit 
einer relativ hohen Reaktionsgeschwindigkeit. Die Thiolgruppe -SH, wie z. B. von einem Alkylthiol, kann die Transferreaktion mit einer hohen Geschwindigkeit $\left(k_{\mathrm{tr}} \approx\right.$ $k_{\mathrm{p}}$ ) eingehen [133]. Es bildet sich dann ein am Schwefel zentriertes Alkylsulfanylradikal als das metastabile Zwischenprodukt. Das Radikal 7 ist allerdings stabiler als das Alkylsulfanylradikal, weil sowohl die benachbarte Carbothioyl-Gruppe als auch der Phenylring eine Resonanzstabilisierung des ungepaarten Elektrons ermöglichen [135]. Die höhere thermodynamische Stabilität des Radikals 7 legt nahe, dass die zugehörige Wasserstoffabstrahierung schneller als die des Alkylthiols verläuft.

Das Radikal 7 kann nun folgende unterschiedliche Reaktionen eingehen:

1. Die Reinitiierung von $\mathbf{7}$, die die klassische Transferreaktion von Dithiobenzoesäure vollendet, ergibt das Radikal 8. Wie das Endprodukt der MichaelAddition von DTBA mit MMA weist das Radikal 8 die charakteristischen Merkmale einer primären Abgangsgruppe auf und ist deshalb nachteilig für einen potentiellen RAFT-Prozess. Dieser Reaktionsweg führt nicht zu einer Bildung eines effektiven RAFT-Agens.

Das Radikal 8 geht höchstwahrscheinlich eine schnelle „back-biting“-Reaktion ein und wandelt sich in ein ringförmiges Radikal 10 um [136]. Die Struktur des Radikals 10 gleicht dem Intermediat in einem RAFT-Gleichgewicht [137]. Dieser Reaktionsschritt wird von sterischen und entropischen Faktoren und von der sehr hohen Stabilität des gebildeten Radikals angetrieben. Das Radikal 10 kann unterschiedliche Resonanzstrukturen haben, weil das ungepaarte Elektron über die beiden Schwefelatome und den Phenylring delokalisiert ist. Diese Faktoren ermöglichen dem Radikal 10 eine lange Lebensdauer und verhindert vollständig einen weiteren Propagierungsschritt. Daher ist das Radikal 10 ein nicht propagierendes Radikal.

Die lange Lebensdauer des Radikals 10 verursacht eine Erhöhung seiner Konzentration, was die Terminierungsreaktion von 10 bedeutender macht. Die Selbstterminierung vom Radikal 10, das sich in verschiedenen Resonanzstrukturen befindet, ergibt die Klasse von möglichen Spezies 11. Zwei Vertreter von diesem Selbstterminierungsprodukt sind in Abbildung 6.10 dargestellt. Dieser selektive Reaktionsweg stimmt mit dem Auftreten des markanten ESI-MSEinzelsignal bei $m / z=529.1$ überein. 


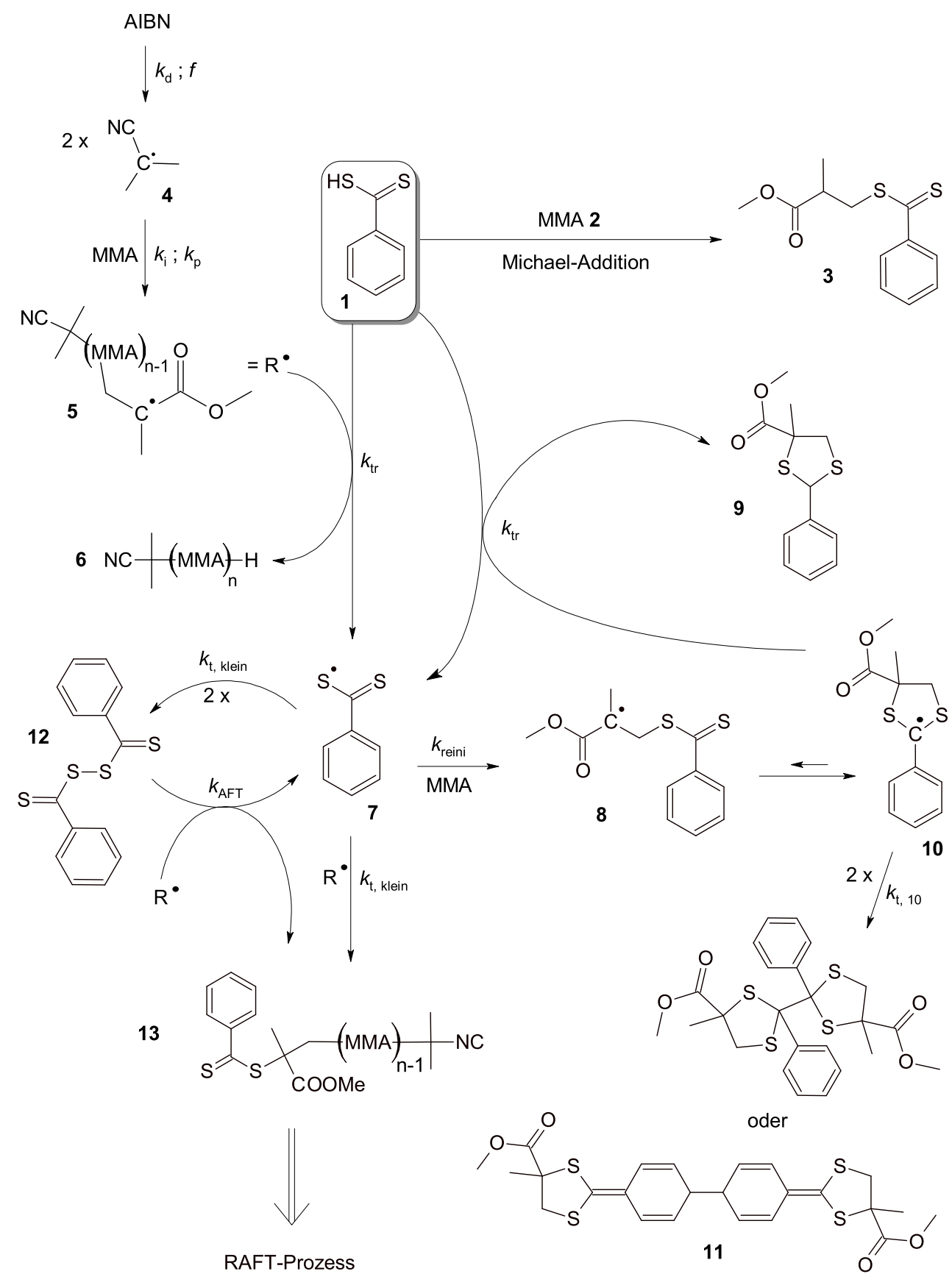

Abbildung 6.10: Schema von dem vorgeschlagenen Reaktionsmechanismus während der Induktion für die Bildung vom RAFT-Agens aus der Dithiobenzoesäure, mit AIBN als Initiator und MMA als Monomer. Nach der Induktionszeit beginnt die Polymerisation mit typischen Merkmalen der kontrollierten RAFT-Polymerisation. 
Zusätzlich zu der beschriebenen Selbstterminierung kann das Radikal 10 einen Wasserstoff von DTBA abstrahieren. Daraus entsteht wieder das Radikal 7 und die Spezies 9, die auch kein effektives RAFT-Agens ist. Dieser Reaktion ergibt aber den notwendigen Reaktionszyklus, bei dem mehr als ein DTBA-Molekül pro Radikal verbraucht wird. Damit kann die Beobachtung erklärt werden, dass die Induktionszeit kürzer ist als die berechnete Zeit nach dem Konzept der klassischen Inhibierung.

2. Das Radikal $\mathbf{7}$ kann mit einem primären oder propagierenden Radikal $\mathrm{R}^{\bullet}$ terminieren und sich in ein Dithiobenzoat mit einer tertiären Abgangsgruppe umwandeln. Dieser Reaktionsweg liefert ein effektives RAFT-Agens 13, das während der Induktionsperiode mit der Hilfe von ESI-MS-Technik nachgewiesen wurde.

3. Die Selbstterminierung des Radikals $\mathbf{7}$ ergibt das Bis(thiobenzoyl)disulfid 12. Diese Verbindung ist in der Literatur als Vorstufe für RAFT-Agens-Synthese bekannt [138]. Sie kann mit einem Radikal $\mathrm{R}^{\bullet}$ in einem Addition-Fragmentierung-Kettentransfer-Reaktionschritt reagieren. Daraus entsteht wieder das Radikal 7 und ein effektives RAFT-Agens 13. Der Nettoeffekt bei diesem Weg zur einem RAFT-Agens ist die Terminierung von 7 mit einem Radikal $R^{\bullet}$. Das Bis(thiobenzoyl)disulfid 12 stellt also ein Reservoir für Radikal 7 bereit. Die Selbstterminierung verhindert somit die unerwünschte Reintiierungsreaktion des Radikals $\mathbf{7}$, indem die Konzentration von $\mathbf{7}$ abgesenkt wird.

Der vorgeschlagene Reaktionsmechanismus stimmt mit allen bisherigen Befunde, wie z. B. die relativ kurze Induktionszeit, die geringe effektive RAFT-Agens-Konzentration und die in den ESI-MS-Spektren auftretenden Spezies, voll überein. Wie die exakten isomeren Strukturen von 9, 10 und 11 aussehen, ist eine in dieser Arbeit nicht tiefer behandelte Frage.

\subsubsection{Modellierung}

Um zu testen, ob der vorgeschlagene Mechanismus plausibel ist, wurde eine kinetische Modellierung der MMA-Polymerisation mit Dithiobenzoesäure bei $60{ }^{\circ} \mathrm{C}$ 
durchgeführt. Die Modellierung basiert auf den Reaktionen, die schematisch in der Abbildung 6.10 und detailliert in der Tabelle 6.4 dargestellt sind.

\begin{tabular}{|c|c|c|c|c|c|c|}
\hline \multicolumn{3}{|c|}{$\begin{array}{l}\text { Bildung von primärem Radikal } \\
\text { AIBN }\end{array}$} & $\longrightarrow$ & \multicolumn{2}{|l|}{$2 \mathrm{IP}^{\bullet}$} & $f \cdot k_{\mathrm{d}}$ \\
\hline $\begin{array}{l}\text { Initiierung } \\
\mathrm{IP}^{\bullet}\end{array}$ & + & MMA & $\longrightarrow$ & \multicolumn{2}{|l|}{ IP-MMA $\bullet$} & $k_{\mathrm{i}}$ \\
\hline \multicolumn{7}{|l|}{ Propagation } \\
\hline IP-MMA• & + & MMA & $\longrightarrow$ & \multicolumn{2}{|l|}{ Makroradikal• } & $k_{\mathrm{p}}$ \\
\hline Makroradikal & + & MMA & $\longrightarrow$ & \multicolumn{2}{|l|}{ Makroradikal ${ }^{\bullet}$} & $k_{\mathrm{p}}$ \\
\hline \multicolumn{7}{|c|}{ Transfer zu DTBA } \\
\hline DTBA & + & $\mathrm{IP}-\mathrm{MMA}^{\bullet}$ & $\longrightarrow$ & IP-MMA-H & $+\mathrm{DTBA}^{\bullet}$ & $k_{\mathrm{tr}}$ \\
\hline DTBA & + & Makroradikal• & $\longrightarrow$ & Polymer & $+\mathrm{DTBA}^{\bullet}$ & $k_{\mathrm{tr}}$ \\
\hline DTBA & + & DTBA-MMA • & $\longrightarrow$ & NonRAFT & $+\mathrm{DTBA}^{\bullet}$ & $k_{\mathrm{tr}}$ \\
\hline \multicolumn{7}{|c|}{ Reaktionen für die Bildung von RAFT-Agens } \\
\hline $\mathrm{DTBA}^{\bullet}$ & + & $\mathrm{IP}^{\bullet}$ & $\longrightarrow$ & RAFT & & $k_{\mathrm{t}, \mathrm{klein}}$ \\
\hline $\mathrm{DTBA}^{\bullet}$ & + & $\mathrm{IP}-\mathrm{MMA}^{\bullet}$ & $\longrightarrow$ & RAFT & & $k_{\mathrm{t}, \mathrm{klein}}$ \\
\hline $\mathrm{DTBA}^{\bullet}$ & + & Makroradikal & $\longrightarrow$ & RAFT & & $k_{\mathrm{t}, \mathrm{klein}}$ \\
\hline $\mathrm{DTBA}^{\bullet}$ & + & DTBA-MMA $\bullet$ & $\longrightarrow$ & RAFT & & $k_{\mathrm{t}, \mathrm{klein}}$ \\
\hline $\mathrm{DTBA}^{\bullet}$ & + & $\mathrm{DTBA}^{\bullet}$ & $\longrightarrow$ & DTBA-DTBA & & $k_{\mathrm{t}, \mathrm{klein}}$ \\
\hline DTBA-DTBA & + & $\mathrm{IP}^{\bullet}$ & $\longrightarrow$ & RAFT & $+\mathrm{DTBA}^{\bullet}$ & $k_{\mathrm{AFT}}$ \\
\hline DTBA-DTBA & + & IP-MMA & $\longrightarrow$ & RAFT & $+\mathrm{DTBA}^{\bullet}$ & $k_{\mathrm{AFT}}$ \\
\hline DTBA-DTBA & + & Makroradikal• & $\longrightarrow$ & RAFT & $+\mathrm{DTBA}^{\bullet}$ & $k_{\mathrm{AFT}}$ \\
\hline \multicolumn{7}{|c|}{ Reaktionen für die Bildung von anderen Dithioverbindungen } \\
\hline $\mathrm{DTBA}^{\bullet}$ & + & MMA & $\longrightarrow$ & DTBA-MMA $\bullet$ & & $k_{\text {reini }}$ \\
\hline DTBA-MMA $\bullet$ & + & DTBA-MMA $\bullet$ & $\longrightarrow$ & $(\mathrm{DTBA}-\mathrm{MMA})_{2}$ & & $k_{\mathrm{t}, 10}$ \\
\hline DTBA-MMA & + & $\mathrm{IP}^{\bullet}$ & $\longrightarrow$ & NonRAFT & & $k_{\mathrm{t}, 10}$ \\
\hline DTBA-MMA & + & IP-MMA $\bullet$ & $\longrightarrow$ & NonRAFT & & $k_{\mathrm{t}, 10}$ \\
\hline DTBA-MMA $\bullet$ & + & Makroradikal & $\longrightarrow$ & NonRAFT & & $k_{\mathrm{t}, 10}$ \\
\hline \multicolumn{7}{|c|}{ Konventionelle Terminierung } \\
\hline IP-MMA• & + & IP-MMA & $\longrightarrow$ & Polymer & & $k_{\mathrm{t}, \text { long }}$ \\
\hline IP-MMA• & + & Makroradikal• & $\longrightarrow$ & Polymer & & $k_{\mathrm{t}, \text { long }}$ \\
\hline Makroradikal & + & Makroradikal & $\longrightarrow$ & Polymer & & $k_{\mathrm{t}, \text { long }}$ \\
\hline
\end{tabular}

Tabelle 6.4: Reaktionsschema für die Modellierung der MMA-Polymerisation mit DTBA, mit IP• 4 , IP-MMA $5(n=1)$, Makroradikal $5(n \succ 1)$, DTBA $\mathbf{1}$, IP-MMAH 6, DTBA • 7, DTBA-MMA $\bullet$, NonRAFT 9, RAFT 13, DTBA-DTBA 12 und (DTBA-MMA) 11.

Diese Modellierung ist nicht entworfen, um die Molmassenverteilung des nach der Induktionszeit gebildeten Polymermaterials zu simulieren, sondern um die Konzentration der betreffenden Spezies zu berechnen. Die Simulation der Molmassenvertei- 
lung des Polymers, das aus einem RAFT-Prozess hervorgeht, wird in der Literatur detailliert beschrieben [34, 81, 139, 38].

Die Input-Parameter, die für die Modellierung der experimentellen Daten verwendet werden, sind in der Tabelle 6.5 angegeben. Zusammengefasst sind diese InputParameter:

- die Einwaagekonzentration von AIBN, DTBA und Monomer,

- die bekannten kinetischen Koeffizienten der konventionellen MMA-Polymerisation mit AIBN als Initiator,

- eine Abschätzung für den Geschwindigkeitskoeffizient der Terminierungsreaktion zwischen kleinen Radikale und

- eine Abschätzung für den Geschwindigkeitskoeffizient von der Addition-Fragmentierung-Kettentransfer-Reaktion der Spezies 12.

\begin{tabular}{lll}
\hline Parameter & Wert bei $60{ }^{\circ} \mathrm{C}$ & Ref. \\
\hline \multicolumn{3}{c}{ Input-Parameter } \\
\hline$[\mathrm{M}]_{0}$ & $9.35 \mathrm{~mol} \cdot \mathrm{L}^{-1}$ \\
$M W_{\text {Monomer }}$ & $100.1 \mathrm{~g} \cdot \mathrm{mol}^{-1}$ & \\
$d$ & 1.7 & {$[133]$} \\
$f$ & 0.8 & {$[140,141]$} \\
$k_{\text {AIBN }}$ & $1.103 \cdot 10^{-5} \mathrm{~s}^{-1}$ & {$[90]$} \\
$k_{\mathrm{i}}$ & $2.857 \cdot 10^{3} \mathrm{~L} \cdot \mathrm{mol}^{-1} \cdot \mathrm{s}^{-1}$ & {$[142]$} \\
$k_{\mathrm{p}}$ & $8.33 \cdot 10^{2} \mathrm{~L} \cdot \mathrm{mol}^{-1} \cdot \mathrm{s}^{-1}$ & {$[143]$} \\
$k_{\mathrm{t}, \mathrm{klein}}$ & $1.0 \cdot 10^{9} \mathrm{~L} \cdot \mathrm{mol}^{-1} \cdot \mathrm{s}^{-1}$ & {$[144]$} \\
$k_{\mathrm{t}, \text { lang }}{ }^{\mathrm{b}}$ & $1.0 \cdot 10^{8} \mathrm{~L} \cdot \mathrm{mol}^{-1} \cdot \mathrm{s}^{-1}$ & {$[145]$} \\
$k_{\mathrm{AFT}}$ & $8.33 \cdot 10^{3} \mathrm{~L} \cdot \mathrm{mol}^{-1} \cdot \mathrm{s}^{-1}$ & {$[146]$} \\
\hline \multicolumn{3}{c}{ angepaste $\operatorname{Ergebnise~}^{-1}$} \\
\hline$k_{\mathrm{tr}}$ & $3.0 \cdot 10^{4} \mathrm{~L} \cdot \mathrm{mol}^{-1} \cdot \mathrm{s}^{-1}$ \\
$k_{\mathrm{reini}}$ & $7.8 \mathrm{~L} \cdot \mathrm{mol}^{-1} \cdot \mathrm{s}^{-1}$ \\
$k_{\mathrm{t}, 10}$ & $\prec 5.0 \cdot 10^{6} \mathrm{~L} \cdot \mathrm{mol}^{-1} \cdot \mathrm{s}^{-1}$ \\
\hline
\end{tabular}

${ }^{a}$ mittlerer Wert für Kettenlängen von $10 \prec n \prec 100$

b abgeschätzt für ein relativ reaktives RAFT-Agens $(C=10)$

Tabelle 6.5: Kinetische Input-Parameter zur Berechnung der theoretischen Molmasse und zur Modellierung der MMA-Polymerisation mit DTBA und AIBN bei $60{ }^{\circ} \mathrm{C}$ sowie die angepassten Ergebnisse. 
Der experimentelle Monomerumsatz (siehe Abbildung 6.2) und die berechnete effektive RAFT-Agens-Konzentration (siehe Abbildung 6.5) in Abhängigkeit von der Reaktionszeit werden gleichzeitig an dieses Modell angepasst, um die Geschwindigkeitskoeffizienten $k_{\mathrm{tr}}, k_{\text {reini }}$ und $k_{\mathrm{t}, 10} \mathrm{zu}$ erhalten. Die angepassten Ergebnisse sind in der Tabelle 6.5 präsentiert.

Die kinetische Modellierung ergibt drei Geschwindigkeitskoeffizienten als angepasste Ergebnisse:

1. Der Geschwindigkeitskoeffizient $k_{\mathrm{tr}}$, der die Wasserstoffabstrahierung der Dithiobenzoesäure beschreibt, wird auf einem Wert von $3.0 \cdot 10^{4} \mathrm{~L} \cdot \mathrm{mol}^{-1} \cdot \mathrm{s}^{-1}$ optimiert. Daraus ergibt sich eine Transferkonstante von $C=k_{\mathrm{tr}} / k_{\mathrm{p}}=36$. Dieser relativ große Wert erscheint beim Vergleich mit der Transferkonstante des Alkylthiols absolut sinnvoll, wie bereits oben ausführlich diskutiert.

Beim Vergleich mit dem Ergebnis von Levesque et al. [136], die im Jahr 1975 für das System DTBA/Styrol eine Transferkonstante von $C=0.06$ bestimmt haben, ist die in dieser Arbeit simulierte Transferkonstante um drei Zehnergrößenordnungen größer. Dieser Unterschied kann nicht nur durch die unterschiedlichen Monomere erklärt werden. Er kann aber in der Bestimmungsmethode seine Begründung finden. Levesque et al. verwendeten die klassische Mayo-Methode, bei der die Molmasse mit der Annahme analysiert wird, dass der Transferprozess irreversibel verläuft. Aber aus der heutigen Sicht mit dem Wissen, dass ein reversibler Addition-Fragmentation-Kettentransfer in der Polymerisation mit DTBA stattfindet, gilt diese Annahme nicht mehr. Die MayoMethode ist deshalb für diesen Fall nicht anwendbar. Levesque et al. haben bereits erkannt, dass das Polymerisationssystem mit DTBA unerwartete kinetische Eigenschaften hatte. Sie haben daher vorsichtshalber von einer „scheinbaren Kettentransferkonstante" berichtet [136].

2. Die beste Anpassung für den Geschwindigkeitskoeffizient $k_{\text {reini }}$, der den Reinitiierungsprozess des Radikal 7 beschreibt, ist $7.8 \mathrm{~L} \cdot \mathrm{mol}^{-1} \cdot \mathrm{s}^{-1}$. Es gibt in der Literatur sehr wenige Daten, mit denen dieser Wert verglichen werden kann. Beim Vergleich mit dem Propagationskoeffizienten $\left(k_{\mathrm{p}}=833 \mathrm{~L} \cdot \mathrm{mol}^{-1} \cdot \mathrm{s}^{-1}\right)$ erkennt man, dass das Radikal 7 sehr langsam ein Monomer addiert. Dieser 
qualitative Befund stimmt gut mit der Beobachtung in „Iniferter"-Systemen überein, in welchem die gleiche Radikalart, ein Carbonylthioylsulfanyl-Radikal, vorkommt [135, 147, 148].

3. Der Geschwindigkeitskoeffizient $k_{\mathrm{t}, 10}$, der die Selbstterminierung des Radikals 10 beschreibt, wird auf einem Wert kleiner als $5.0 \cdot 10^{6} \mathrm{~L} \cdot \mathrm{mol}^{-1} \cdot \mathrm{s}^{-1}$ bestimmt. Kleinere Werte für $k_{\mathrm{t}, 10}$ ändern den Simulationsausgang nicht signifikant. Deshalb wird nur die obere Grenze für diesen Wert berichtet. Trotzdem ist er interessanterweise mindestens zwei Zehnerpotenzen kleiner als der typische Wert für die Terminierung von zwei kleinen Radikalen. Dieses Ergebnis stimmt mit der obigen Überlegung überein, dass das Radikal 8 sich sehr schnell durch die „back-biting“-Reaktion in die Spezies 10 umwandelt. Das relativ stabile Radikal 10 hat wegen der starken Delokalisierung des ungepaarten Elektrons eine relativ kleine Terminierungsaktivität.

Die simulierten Monomerumsatz-Reaktionszeit-Kurven in Abbildung 6.2 zeigen die gute Beschreibung der MMA-Polymerisation in der Induktionszeit. Die UmsatzZeit-Kurve weicht leicht von den experimentellen Punkten ab, weil dieses Modell den Retardierungseffekt während der Polymerisationsphase eines RAFT-Prozesses nicht berücksichtigt. Die simulierte Induktionszeit hat aber eine sehr gute Übereinstimmung mit den beobachteten Werten, wie die Abbildung 6.3 deutlich zeigt. Dieser Befund stärkt die Richtigkeit des vorgeschlagenen Mechanismus.

Abbildung 6.11 gibt die experimentelle und simulierte Konzentration des effektiven RAFT-Agens als eine Funktion der Reaktionszeit. Die Modellierung zeigt die Situation gut wieder, dass nur ein relativ kleiner Teil von ca. $15 \%$ der eingesetzten Dithiobenzoesäure ins effektive RAFT-Agens umgewandelt wurde.

Eine Abweichung der simulierten Kurve von den experimentellen Ergebnissen kann auf experimentelle Unsicherheiten, Fehler bei der Abschätzung der InputParameter oder ein nicht vollständiges Reaktionsmodell beruhen. Eine weitere Verfeinerung des Reaktionsmodells, z. B. unter der Einbeziehung von zusätzlichen Reaktionen, scheint aufgrund der momentan erhältlichen experimentellen Daten nicht sinnvoll. Zusätzliche zeitabhängige Konzentrationensverläufe der einzelnen Spezies wären nötig, um die komplexe Kinetik während der Induktionsperiode der MMAPolymerisation mit DTBA noch exakter zu beschreiben. 


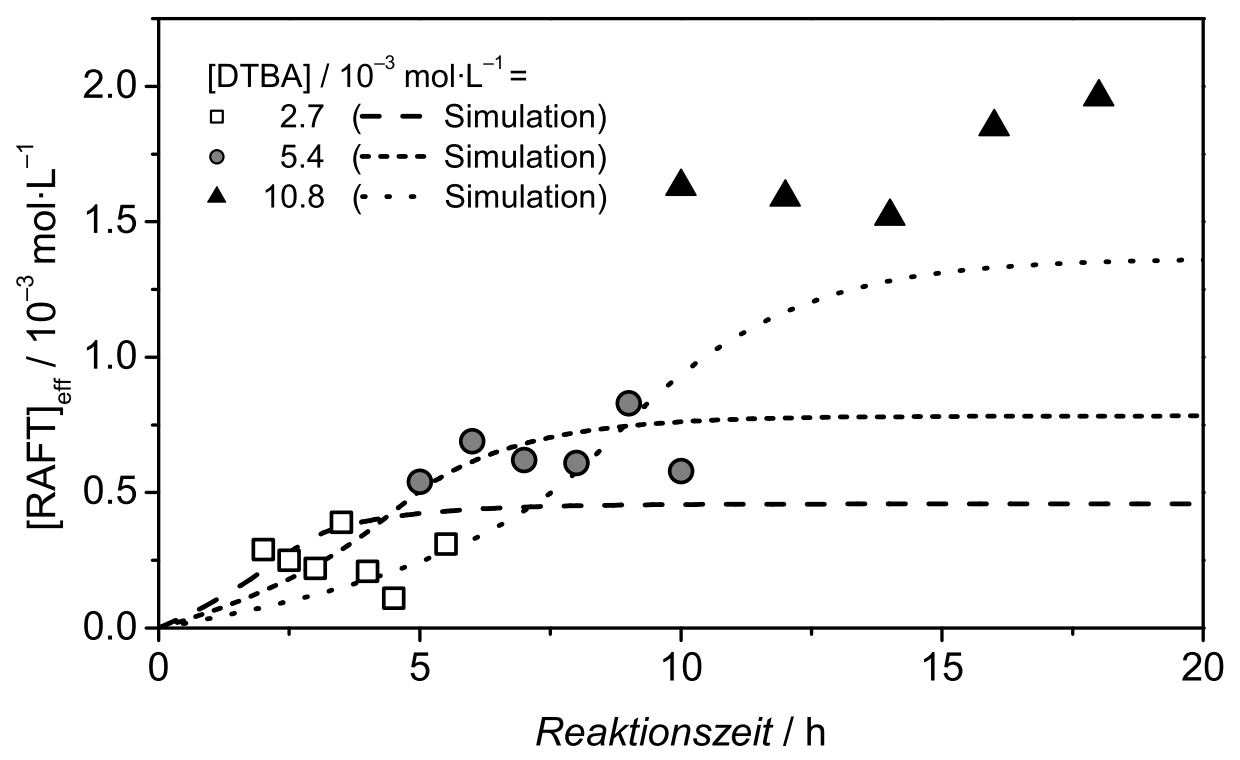

Abbildung 6.11: Experimentelle und simulierte Werte der effektiven RAFT-AgensKonzentration $[\mathrm{RAFT}]_{\mathrm{eff}}$ in Abhängigkeit von der Reaktionszeit. Die MMA-Polymerisation wurde bei $60{ }^{\circ} \mathrm{C}$ mit verschiedenen DTBA-Konzentrationen und $4.9 \cdot 10^{-3} \mathrm{~mol} \cdot \mathrm{L}^{-1}$ AIBN durchgeführt.

Um die Komplexität in der Induktionsperiode zu verdeutlichen, soll im Folgenden ein hypothetischer Mechanismus kurz diskutiert werden. In dieser Reaktionssequenz ergibt die Addition eines propagierenden Radikals 5 mit DTBA ein metastabiles Zwischenprodukt 14; dieser Reaktionstypus ist für seinen schnellen Verlauf bekannt [149]. Die Spezies 14 disproportioniert mit einem anderen propagierenden Radikal. Daraus entstehen das effektive RAFT-Agens 13 und das tote Polymer 6. Die gleichen Produkte sind in dem obigen Reaktionsschema in Abbildung $6.10 \mathrm{zu}$ finden, die aber einen anderen Mechanismus zeigt. Dieser Reaktionssequenz fehlt jedoch ein notwendiger Reaktionszyklus, bei welchem DTBA schneller als der klassische Inhibitor verbraucht wird. Außerdem kann sie die Entstehung der Spezies 11 nicht erklären. Diese Reaktionen können deshalb nicht der einzige Grund für die Bildung des effektiven RAFT-Agens bei der MMA-Polymerisation mit DTBA sein. 


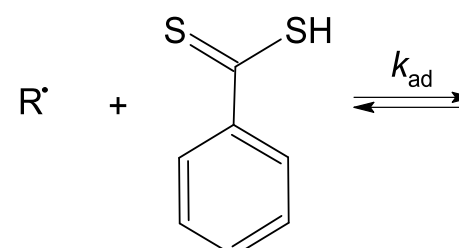

5

1<smiles>[R]S[C](S)c1ccccc1</smiles>

14

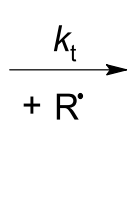<smiles>[R]SC(=S)c1ccccc1</smiles>

13

6

Abbildung 6.12: Eine hypothetische Reaktionssequenz, die zu das effektive RAFTAgens 13 führt.

\subsubsection{Initiator-Cocktail}

Auf der Grundlage des Reaktionsschemas und der Ergebnisse der Simulation wird ein Polymerisationsprotokoll entwickelt, mit dem die Induktionszeit stark verkürzt wird und die relative Menge des effektiven RAFT-Agens deutlich erhöht wird. Dazu wird ein Initiator-Cocktail eingesetzt, der einen schnell zerfallenden und einen langsam zerfallenden Initiator enthält.

Bei der Verwendung eines Initiator-Cocktails, in welchem ein Initiator schnell zerfällt, wird eine große Radikalmenge zu Beginn der Polymerisation in das System eingespeist. Diese große Menge an Radikalen verursacht eine schnelle Umwandlung von DTBA ins effektive RAFT-Agens. Nach der kurzen Umwandlungsperiode ist der schnell zerfallende Initiator komplett verbraucht. Ein zweiter, langsam zerfallender Initiator wird dann eingesetzt, um die anschließende RAFT-Polymerisation aufrecht $\mathrm{zu}$ erhalten.

Die Verwendung solch eines Initiator-Cocktails ist in Abbildung 6.13 demonstriert, in welcher der Monomerumsatz der MMA-Polymerisation mit DTBA bei $100{ }^{\circ} \mathrm{C}$ gegen die Reaktionszeit aufgetragen ist. Beim ausschließlichen Einsatz eines langsam zerfallenden Initiators wie ACCN, der eine Halbwertszeit von 1.9 Stunden bei $100{ }^{\circ} \mathrm{C}$ hat, beobachtet man eine typische Induktionsphase von zwei Stunden für die MMA-Polymerisation mit DTBA. Durch die Zugabe von AIBN, einem schnell zerfallenen Initiator mit einer Halbwertszeit von 0.11 Stunden bei $100{ }^{\circ} \mathrm{C}$, verschwindet die Induktionsphase. Durch die Reduzierung der Induktionszeit kann man bei gleicher Reaktionszeit einen viel höheren Monomerumsatz mit dem Initiator- 


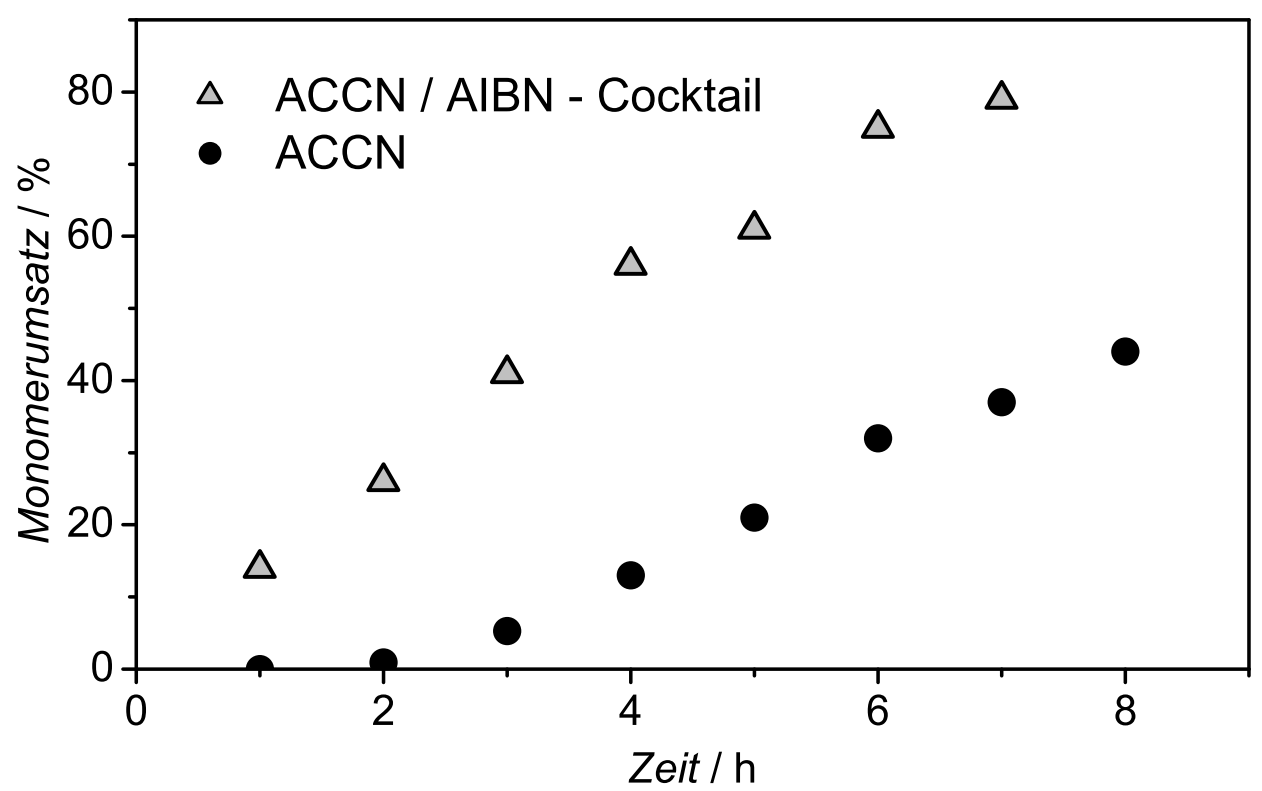

Abbildung 6.13: Monomerumsatz in Abhängigkeit von der Reaktionszeit für die MMAPolymerisation bei $100{ }^{\circ} \mathrm{C}$ mit DTBA $\left(33.0 \cdot 10^{-3} \mathrm{~mol} \cdot \mathrm{L}^{-1}\right)$, initiiert durch ACCN (5.0 $\left.10^{-3} \mathrm{~mol} \cdot \mathrm{L}^{-1}\right)$ und durch einen Initiator-Cocktail $\left(5.0 \cdot 10^{-3} \mathrm{~mol} \cdot \mathrm{L}^{-1}\right.$ ACCN und 5.2 . $\left.10^{-3} \mathrm{~mol} \cdot \mathrm{L}^{-1} \mathrm{AIBN}\right)$.

Cocktail als nur mit ACCN erreichen. Diese Beobachtungen können dem oben beschriebenen Simulationsmodell vorhergesagt werden.

Die Entwicklung der mittleren Molmasse mit dem Monomerumsatz und die Polydispersität des gebildeten Polymers sind ähnlich für das ACCN- und ACCN/AIBNSystem (siehe Abbildung 6.14). Diese Beobachtung weist darauf hin, dass die effektive Kontrolle der Molmasse durch die Verwendung eines Initiator-Cocktails nicht negativ beeinflusst wird. Der absolute Wert für die mittlere Molmasse ist bei dem ACCN/AIBN-Cocktail-System deutlich kleiner, was auf eine höhere Konzentration des in situ gebildeten effektiven RAFT-Agens hindeutet. Dies ist mit der Hilfe des kinetischen Modells vorhersehbar.

Das vorgestellte Protokoll ist im Wesentlichen eine in situ Synthese des RAFTAgens über eine Radikalreaktionssequenz, wonach die günstige Situation einer vernachlässigbaren Polymerisationsaktivität während der Induktionsphase ausgenutzt wird. Diese eigene Polymerisationsbremse wird dann losgelassen, nachdem die kom- 


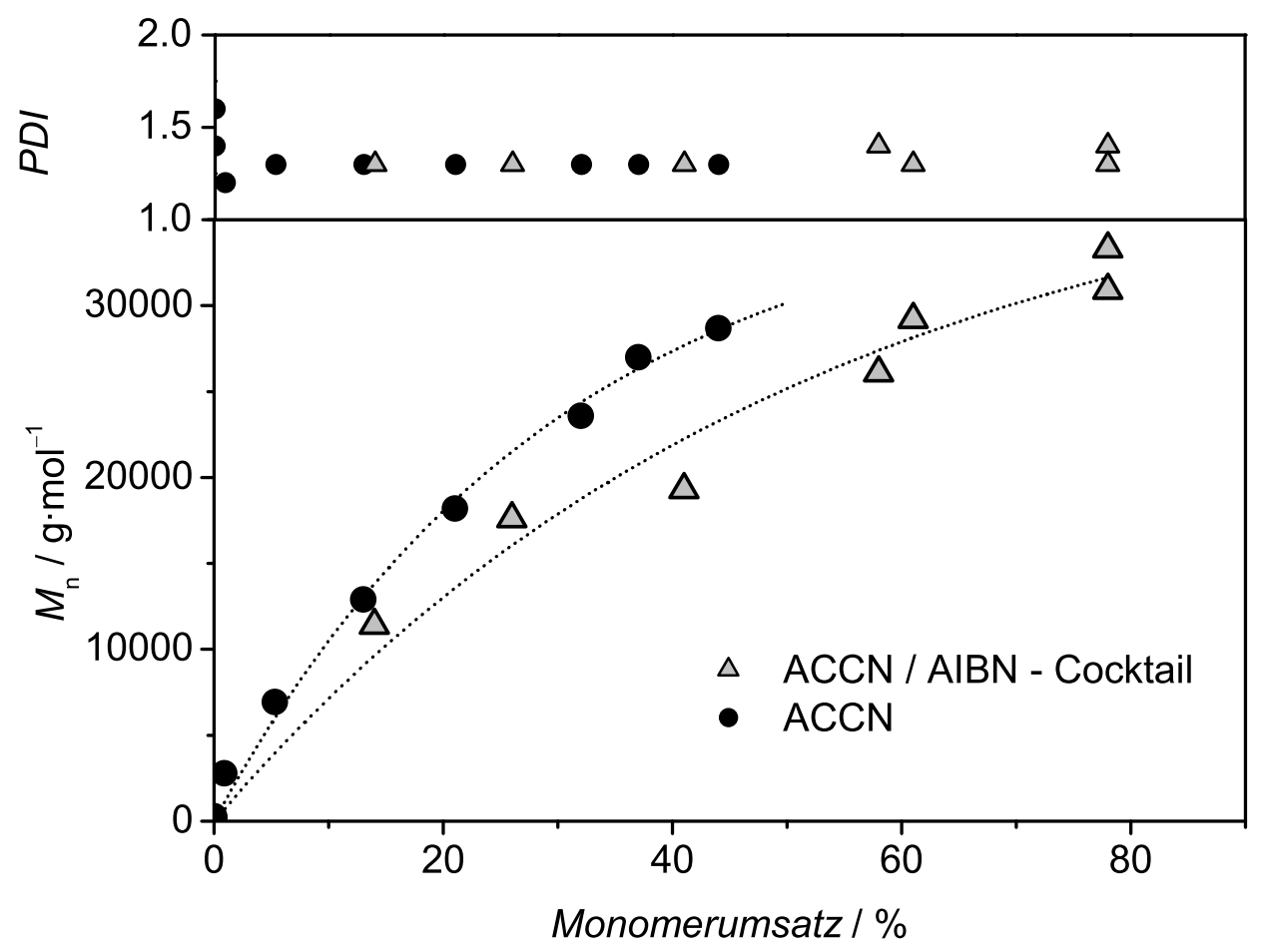

Abbildung 6.14: Auftragung des Zahlenmittels der Molmassen $M_{\mathrm{n}}$ und Polydispersität PDI gegen den Monomerumsatz für die Polymerisation von MMA bei $100{ }^{\circ} \mathrm{C}$ mit DTBA $\left(33.0 \cdot 10^{-3} \mathrm{~mol} \cdot \mathrm{L}^{-1}\right)$, initiiert durch $\operatorname{ACCN}\left(5.0 \cdot 10^{-3} \mathrm{~mol} \cdot \mathrm{L}^{-1}\right)$ und durch einen InitiatorCocktail $\left(5.0 \cdot 10^{-3} \mathrm{~mol} \cdot \mathrm{L}^{-1}\right.$ ACCN und $5.2 \cdot 10^{-3} \mathrm{~mol} \cdot \mathrm{L}^{-1}$ AIBN). Die gepunkteten Linien sind Leitlinien.

plette Menge von DTBA verbraucht ist. Dadurch entsteht ein relativ gut definierter Startpunkt der kontrollierten Polymerisation, und ein Polymer mit niedriger Polydispersität wird gebildet.

\subsubsection{Retardierungseffekt}

Wie in Kapitel 2 bereits diskutiert, wird die Retardierung der Polymerisationsgeschwindigkeit in vielen RAFT-Polymerisationen beobachtet. Der Retardierungseffekt nimmt mit einer steigenden Stabilität des intermediären RAFT-Radikals und mit steigender RAFT-Agens-Konzentration zu [125]. 


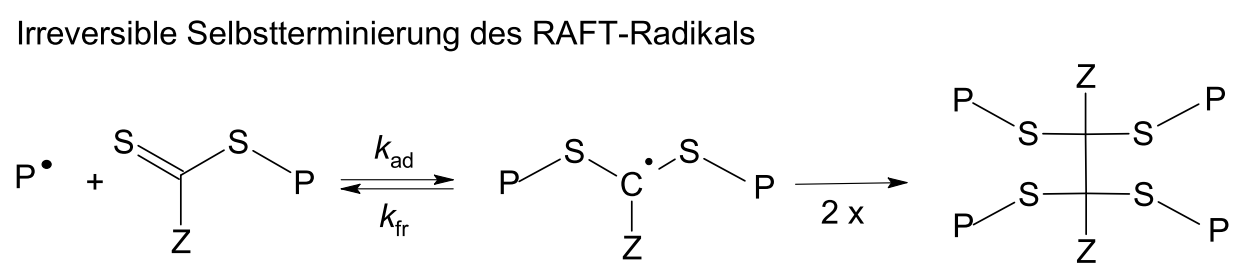

4-Arm-Sterne

Bildung der Spezies 11<smiles>COC(=O)[C](C)CSC(=S)c1ccccc1</smiles>

8

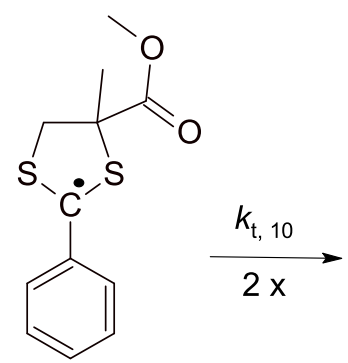

10

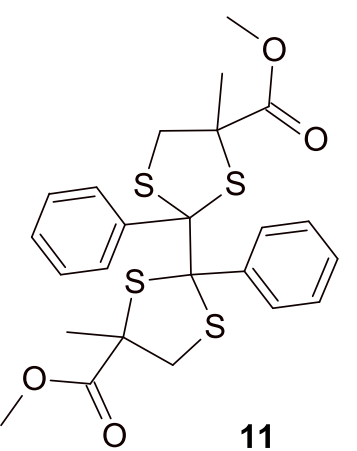

11

Abbildung 6.15: Vergleich der Bildungswege für Spezies 11 und für das hypothetische 4-Arm-Stern-Polymer.

Zurzeit gibt es zwei Erklärungsansätze, die den experimentellen Monomerumsatzverlauf mit der Reaktionszeit gut beschreiben können: die irreversible Terminierung und die langsame Fragmentierung des Intermediats. Viele experimentelle Daten sprechen für die irreversiblen Terminierung, doch ein Hauptargument gegen diese Theorie ist, dass bis heute bei gewöhnlichen RAFT-Polymerisationen keine aus der irreversiblen Terminierung stammende Produkte, z. B. Drei-Arm-Sterne oder Vier-Arm-Sterne ohne Zweifel nachgewiesen werden können [32, 39].

Wenn man den Mechanismus der irreversiblen Terminierung, die zur Bildung des 4-Arm-Sternpolymers führt, mit der Bildung der Spezies 11 in der MMAPolymerisation mit DTBA vergleicht, ist eine Analogie deutlich erkennbar (siehe Abbildung 6.15). Beide Wege verlaufen über das intermediäre Dithio-Radikal und über die Selbstterminierung zur Bildung eines 4-Arm-Sterns.

Bei der MMA-Polymerisation mit DTBA ist das entstehende Dithio-Radikal 10 wegen seiner extrem hohen Resonanzstabilisierung sehr stabil, sodass seine Konzentration sich stark erhöht und seine Selbstterminierung wahrscheinlicher wird. Als 
Folge steigt die Menge des Produkts 11 aus dieser Selbstterminierung an und kann durch die ESI-MS-Technik detektiert werden.

Auch wenn die Spezies 11 kein echtes 4-Arm-Sternpolymer ist, hat seine Existenz bewiesen, dass die Bildung des 4-Arm-Sternpolymers über die Selbstterminierung des intermediären RAFT-Radikals möglich ist. Das ist ein weiterer experimenteller Beweis für die irreversible Terminierung als die Hauptursache des Retardierungseffekts.

\subsection{Schlussfolgerung}

Die MMA-Polymerisation mit DTBA zeigt eine ausgeprägte Induktionsphase, in der das eingesetzte DTBA verbraucht wird und sich in ein effektives RAFT-Agens verwandelt. Dieses in situ gebildete RAFT-Agens bewirkt eine kontrollierte Polymerisation nach dem Ablauf der Induktionszeit. Die Umwandlung von DTBA in das effektive RAFT-Agens verläuft über eine Wasserstoffabstrahierung von DTBA und eine anschließende Terminierung des gebildeten Phenylcarbonothioylsulfanylradikal $(P h-C(=S) S \bullet)$ mit einem propagierenden Radikal, wobei ein Dithioester mit einer tertiären Abgangsgruppe entsteht. Die Reinitiierung des Carbothioylsulfanylradikals ergibt eine Spezies, die nicht als RAFT-Agens wirken kann. Eine weitere Addition eines Monomers ist wegen der Stabilität des gebildeten Radikals nicht mehr möglich, was die Induktion der Polymerisation verursacht. Die Induktion dauert so lange, bis die eingesetzte DTBA-Menge vollständig verbraucht ist. Die Selbstterminierung des gebildeten Dithio-Radikals liefert einen Beweis für die Theorie der irreversiblen Terminierung, die als die Hauptursache für die Retardierung in vielen RAFT-Polymerisationen gilt.

Der vorgeschlagene Mechanismus für die Umwandlung von DTBA wird durch eine computerunterstützte Simulation überprüft. Die kinetische Modellierung bietet Informationen für die Geschwindigkeitskoeffizienten der Wasserstoffabstrahierung von DTBA und der Reinitiierung des Phenylcarbonothioylsulfanylradikals. Das Modell stimmt (a) mit allen durch die ESI-MS-Technik nachgewiesenen Spezies, (b) mit der experimentellen Induktionszeit und (c) mit der experimentellen Konzentrationen des effektiven RAFT-Agens überein. Auf der Grundlage des Mechanismus und der 
Simulation wird ein Protokoll für die Verwendung eines Initiator-Cocktails entwickelt, um die Induktionszeit zu verkürzen und um die Menge des in situ gebildeten RAFT-Agens deutlich zu erhöhen. Dieses Protokoll ist eine attraktive Alternative zur klassischen RAFT-Polymerisation von MMA, weil DTBA mit erheblich weniger Mühe und Kosten als die konventionellen RAFT-Agenzien erhältlich ist. 


\section{Kapitel 7}

\section{Block-, Stern-, Kamm- und Netzwerk-Polymere}

In diesem Kapitel soll eine Brücke ausgehend von Homopolymeren über Block-, Stern- Kamm- und Netzwerk-Polymere bis hin zu gebundenen Polymeren gebaut werden. Mit einer einfachen, allgemein anwendbaren Technik werden all diese unterschiedlichen Zusammensetzungen, Topologien und Funktionalitäten der Polymere entwickelt.

\subsection{Einführung}

Die Entdeckung der kontrollierten Polymerisationstechnik hat die radikalische Polymerisation revolutioniert. Diese Technik erlaubt eine sehr effiziente Kontrolle der Polymerisation im Hinblick auf die Molmasse und die makromolekulare Architektur. Demzufolge ist die Herstellung von synthetischen Polymeren mit niedriger Polydispersität und komplexer Mikrostruktur für vielfältige Anwendungen relativ einfach durchzuführen [4]. Beispiele sind hochverzweigte, Stern-, Kamm- und Blockcopolymere mit unterschiedlichen Zusammensetzungen und Funktionalitäten (siehe Abbildung 2.2 in Kapitel 2).

Die RAFT-Polymerisation, die von Rizzardo et al [19, 27, 28] im Jahr 1999 entdeckt wurde, ist die neueste Entwicklung in diesem Forschungsfeld. Sie ist wohl die vielfältigste kontrollierte Polymerisationsmethode bezogen auf unterschiedliche Monomere und Reaktionsbedingungen. Deshalb kam es in der Literatur seit ihrer 
Entdeckung zu einer rasant ansteigenden Anzahl der Veröffentlichungen zum Thema RAFT-Polymerisation.

Ein Nachteil der RAFT-Polymerisationstechnik ist das RAFT-Agens selbst, welches noch nicht kommerziell zu erwerben ist. Zwar lässt sich der RAFT-Prozess auf eine große Auswahl an Monomere und an polymeren Architekturen anwenden, doch müssen die verwendeten RAFT-Agenzien auf das verwendete Monomer und auf die jeweilige gewünschte Struktur angepasst werden. Es existiert noch kein allgemein gültiges RAFT-Agens für alle Anwendungszwecke.

Das Ziel dieser Arbeit ist es, zahlreiche Eigenschaften von Polymeren kontrolliert beeinflussen zu können, um polymere Materialien gezielt an bestimmte Anwendungen anpassen zu können. In diesem Kapitel werden die homogenen RAFTPolymerisationen mit verschiedenen Trithiocarbonate dargestellt, die einen Beitrag zur Entwicklung neuartiger Polymermaterialen leisten. Unterschiedliche Strategien und Vorgehensweisen, die sich aus den Arbeiten am heterogenen RAFT-Polymerisationssystem hergeben haben, werden hier zusammengefasst.

\subsection{Experiment}

\subsubsection{Synthese von RAFT-Agenzien}

Die in diesem Kapitel diskutierten RAFT-Agenzien sind in Abbildungen 7.1 und 7.2 dargestellt. Ihre Synthese verläuft analog dem in Abbildung 7.4 gezeigten Verfahren mit den entsprechenden Edukten und Stöchiometrien.

Die Synthese der Trithiocarbonate ist relativ einfach durchzuführen, und die meisten Edukte sind kostengünstig zu erwerben. Die Selektivität der Reaktion ist hoch, was zu einer hohen Ausbeute der Produkte führt. Aber für die RAFT-Agenzien mit Methoxysilangruppen ist die Ausbeute relativ niedrig, da bei der Reinigung der Produkte durch Säulenchromatographie die reaktiven Methoxysilangruppen am Kieselgel haften bleiben können, was zum Verlust an Produkt führt. Deshalb muss die Reinigung der Produkte zügig durchgeführt werden, um möglichst hohe Produktausbeuten zu erhalten.

Abbildung 7.1 zeigt die chemischen Strukturen der monofunktionellen Trithiocarbonate. Sowohl die Abgangsgruppe $\mathrm{R}$ als auch die stabilisierende Gruppe Z des 

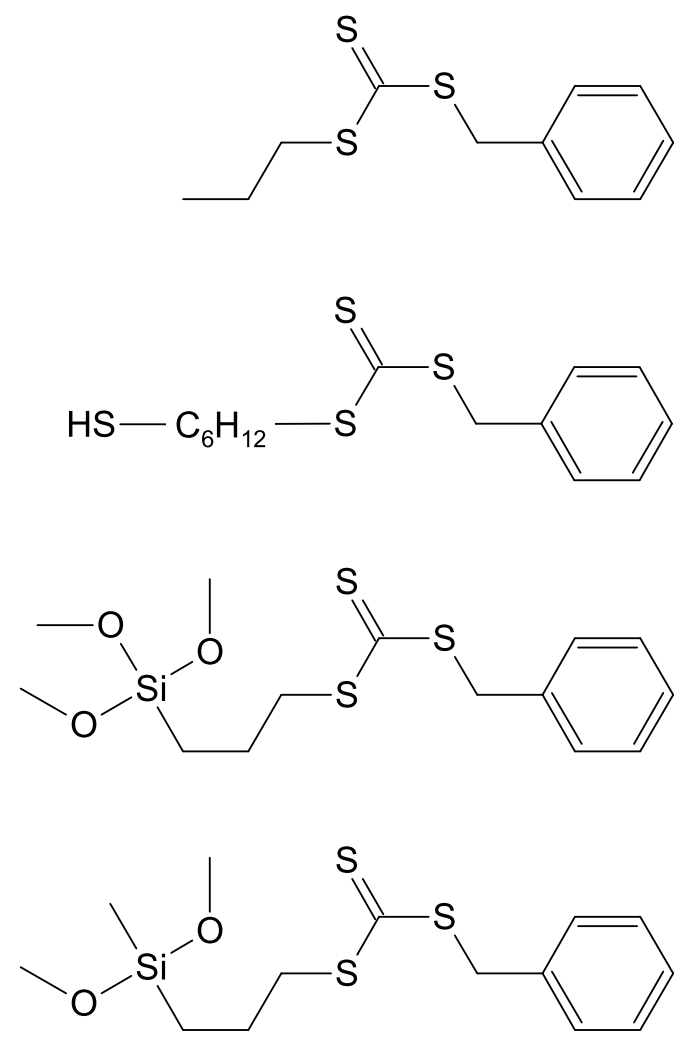

5
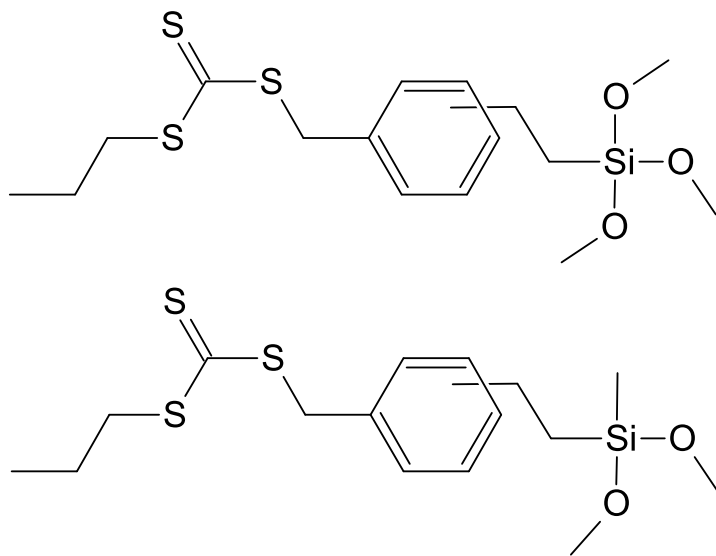

6

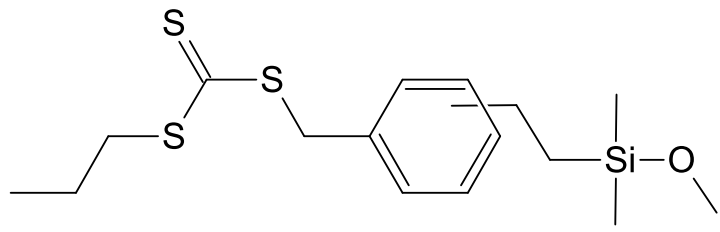

7

Abbildung 7.1: Synthetisierte monofunktionelle Trithiocarbonate. 


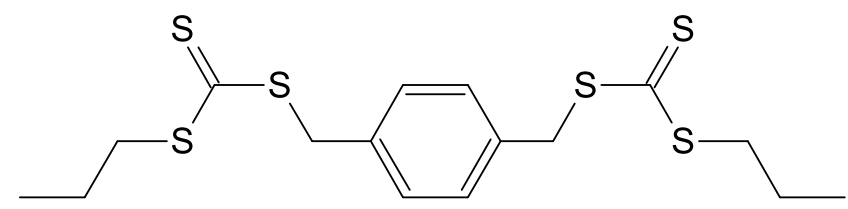

8

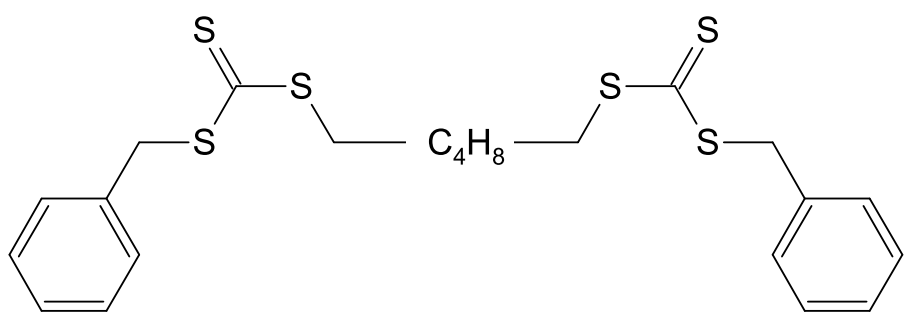

9<smiles>CO[Si](CCCSC(=S)SCc1ccc(CSC(=S)SCCC[Si](OC)(OC)OC)cc1)(OC)OC</smiles>

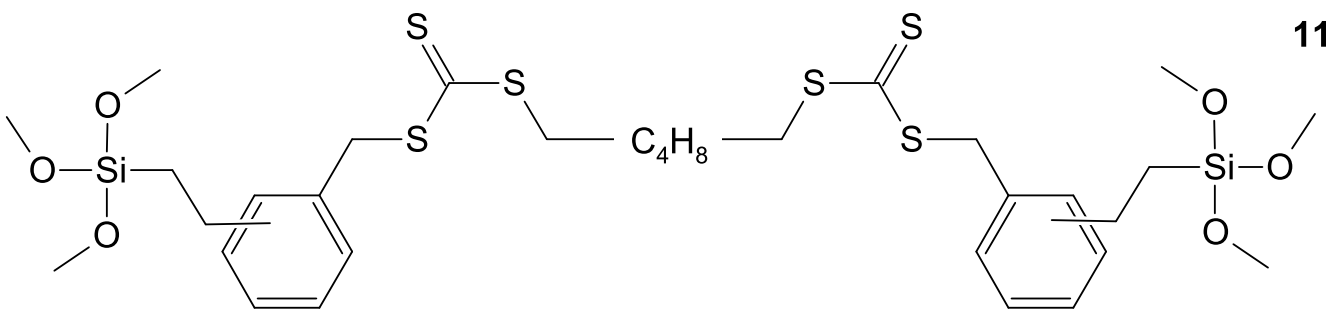

Abbildung 7.2: Synthetisierte bifunktionelle Trithiocarbonate.

RAFT-Agens können eine Methoxysilangruppe enthalten. Dabei kann die reaktive Methoxysilangruppe, die an der Oberfläche eines Substrats chemisch binden kann, eine bis drei Methoxygruppen tragen. In Abbildung 7.2 sind die korrespondierenden bifunktionellen Trithiocarbonate dargestellt.

Abbildung 7.3 zeigt exemplarisch das ${ }^{1} \mathrm{H}$ - und ${ }^{13} \mathrm{C}-\mathrm{NMR}-S p e k t r u m$ von dem isolierten, chemisch reinen Produkt 10, das ein bifunktionelles Trithiocarbonat mit zwei Trimethoxysilangruppen darstellt. Durch die symmetrische Struktur von 10 sind die Spektren trotz der Molekülgröße relativ unkompliziert, was eine einfache Interpretation ermöglicht. 

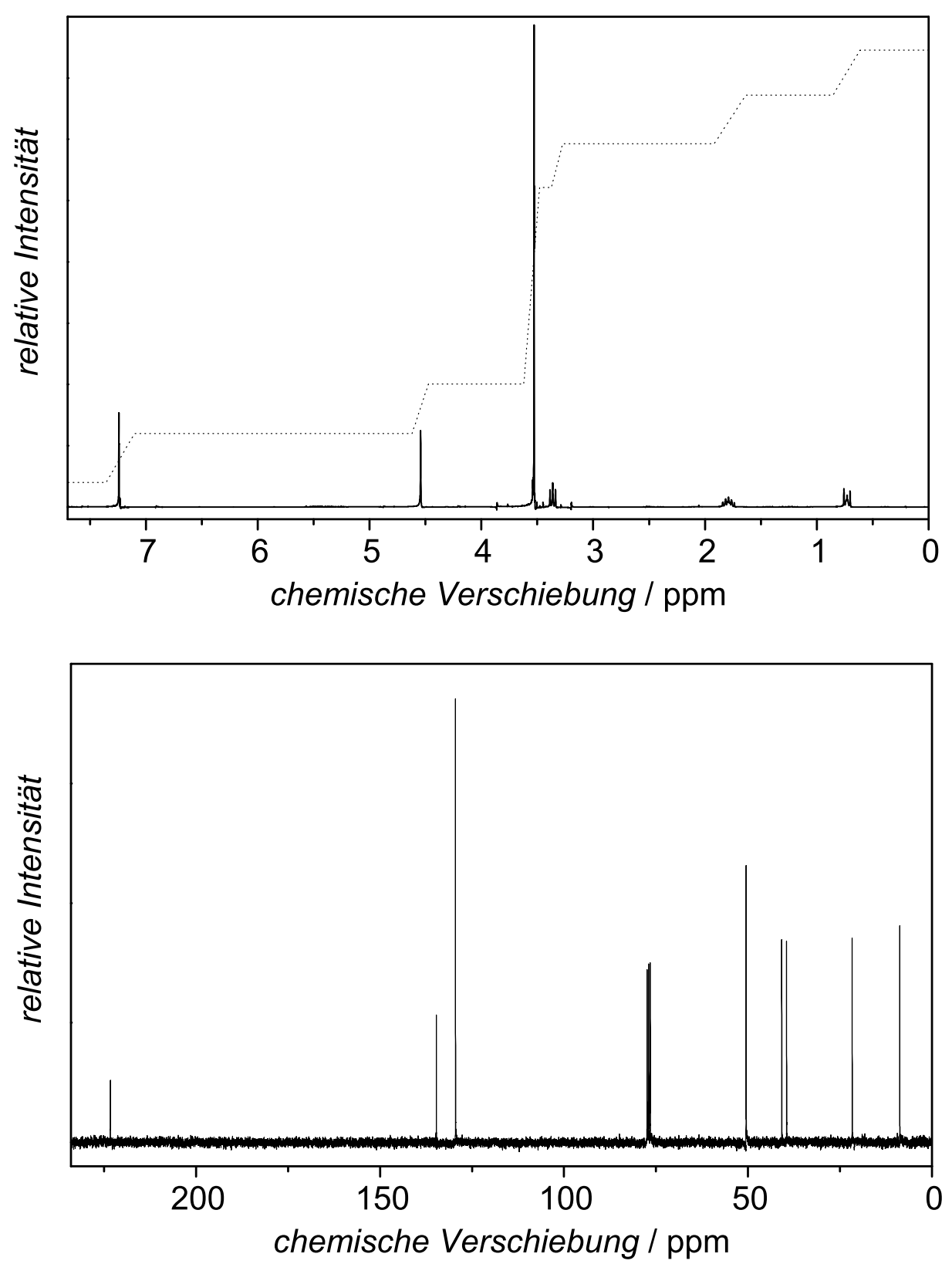

Abbildung 7.3: ${ }^{1} \mathrm{H}-$ und ${ }^{13} \mathrm{C}-\mathrm{NMR}-$ Spektrum von 10.

Propyl-(phenylmethyl)-trithiocarbonat $\mathbf{1}$

Zu einer 30 \%igen Lösung von Natriummethanolat (18.0 g, $0.10 \mathrm{~mol}$ ) in Methanol $(30 \mathrm{~mL})$ wurde tropfenweise Propanthiol (7.6 g, $0.10 \mathrm{~mol})$ unter einer StickstoffSchutzgasatmosphäre bei $0{ }^{\circ} \mathrm{C}$ hinzugegeben. Die Reaktionslösung wurde zwei Stunden bei Zimmertemperatur gerührt. Danach wurde Schwefelkohlenstoff (7.6 g, 0.10 


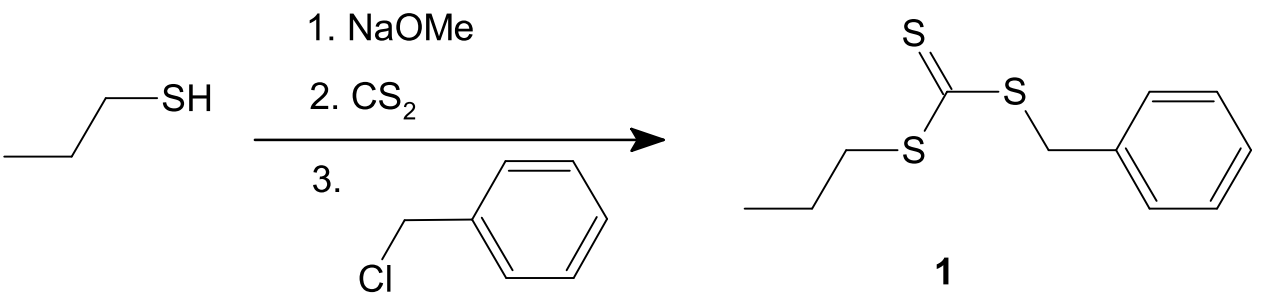

Abbildung 7.4: Schematische Darstellung der Synthese von Propyl-(phenylmethyl)trithiocarbonat 1 aus Propanthiol. Andere Trithiocarbonate wurden analog mit ähnlichen Edukten und verschiedenen Stöchiometrien synthetisiert.

mol) zu der Lösung hinzugetropft. Nach weiteren drei Stunden wurde Benzylchlorid (12.6 g, 0.10 mol) unter Rühren zu der Lösung dazugegeben. Die Reaktionslösung wurde dann für 12 Stunden bei Zimmertemperatur gerührt.

Nachdem das Lösungsmittel unter vermindertem Druck entfernt wurde, wurde das Produkt über Säulenchromatographisch gereinigt (Silicagel, Pentan; $R_{\mathrm{f}}=$ 0.17). Man erhält das Propyl-(phenylmethyl)trithiocarbonat 1 als leicht gelbliches Öl (19.0 g, $0.078 \mathrm{~mol}, 78 \%$ ).

${ }^{1} \mathrm{H}-\mathrm{NMR}\left(300 \mathrm{MHz}, \mathrm{CDCl}_{3}\right), \delta(\mathrm{ppm}): 1.0\left(\mathrm{t}, 3 \mathrm{H}, \mathrm{CH}{ }_{3}\right), 1.7$ (quintett, $2 \mathrm{H}, \mathrm{CH}_{2^{-}}$ $\left.\mathrm{CH}_{3}\right), 3.4\left(\mathrm{t}, 2 \mathrm{H}, \mathrm{CH}_{2}-\mathrm{CH}_{2}-\mathrm{CH}_{3}\right), 4.6\left(\mathrm{~s}, 2 \mathrm{H}, \mathrm{CH}_{2}\right), 7.2-7.4\left(\mathrm{~m}, 5 \mathrm{H}, \mathrm{C}_{6} \mathrm{H}_{5}\right)$.

${ }^{13} \mathrm{C}-\mathrm{NMR}\left(300 \mathrm{MHz}, \mathrm{CDCl}_{3}\right), \delta(\mathrm{ppm}): 13.4\left(\mathrm{CH}_{2}-\mathrm{CH}_{2}-\mathrm{CH}_{3}\right), 21.5\left(\mathrm{CH}_{2}-\mathrm{CH}_{2}-\right.$ $\left.\mathrm{CH}_{3}\right), 38.8\left(\mathrm{CH}_{2}-\mathrm{CH}_{2}-\mathrm{CH}_{3}\right), 41.3\left(\mathrm{C}_{6} \mathrm{H}_{5}-\mathrm{CH}_{2}-\mathrm{S}\right), 127.7,128,6,129,2,135.0\left(C_{6} \mathrm{H}_{5}\right)$, $223.7(C(=\mathrm{S}) \mathrm{S})$.

\section{6-Thiolhexyl-(phenylmethyl)-trithiocarbonat $\mathbf{2}$}

Die synthetische Prozedur von 2 ist identisch mit der von 1, außer dass anstatt von Propanthiol 1,6-Hexandithiol (15.0 g, $0.10 \mathrm{~mol}$ ) eingesetzt wurde. Das Produkt 2 wurde mit Hilfe der Säulenchromatographie (Silicagel, Dichlormethan, $R_{\mathrm{f}}=$ $0.67)$ vorgereinigt und in Heptan als gelblich leuchtender Kristall ausgefällt (7.1 g, $0.0224 \mathrm{~mol}, 22.4 \%)$.

${ }^{1} \mathrm{H}-\mathrm{NMR}\left(300 \mathrm{MHz}, \mathrm{CDCl}_{3}\right), \delta(\mathrm{ppm}): 1.2(\mathrm{~m}, 1 \mathrm{H}, \mathrm{SH}), 1.3\left(\mathrm{~m}, 4 \mathrm{H}, 2 \times \mathrm{CH}_{2}\right)$, $1.6\left(\mathrm{~m}, 4 \mathrm{H}, 2 \times \mathrm{CH}_{2}\right), 2.4\left(\mathrm{~m}, 2 \mathrm{H}, \mathrm{CH}_{2}-\mathrm{SH}\right), 3.3\left(\mathrm{~m}, 2 \mathrm{H}, \mathrm{CH}_{2}-\mathrm{S}-\mathrm{CS}_{2}\right), 4.6(\mathrm{~s}, 2 \mathrm{H}$, 
$\left.\mathrm{CH}_{2}\right), 7.2-7.4\left(\mathrm{~m}, 5 \mathrm{H}, \mathrm{C}_{6} H_{5}\right)$.

${ }^{13} \mathrm{C}-\mathrm{NMR}\left(300 \mathrm{MHz}, \mathrm{CDCl}_{3}\right), \delta(\mathrm{ppm}): 24.1,27.4,27.5,27.9\left(4-\times \mathrm{CH}_{2}\right), 33.4$, 36.4, $41.0\left(3-\times \mathrm{CH}_{2}-\mathrm{S}\right), 127.6,128.5,129.1,134.9\left(C_{6} \mathrm{H}_{5}\right), 223.5(C(=\mathrm{S}) \mathrm{S})$.

\section{3-(Trimethoxysilan)propyl-(phenylmethyl)-trithiocarbonat $\mathbf{3}$}

Die synthetische Prozedur von $\mathbf{3}$ ist identisch mit der von $\mathbf{1}$, außer dass anstatt von Propanthiol 3-Mercaptopropyltrimethoxysilan (19.6 g, $0.10 \mathrm{~mol}$ ) eingesetzt wurde. Das Produkt wurde mit Hilfe der Säulenchromatographie (Silicagel, Pentan : Dichlormethan $\left.=9: 1, R_{\mathrm{f}}=0.47\right)$ gereinigt. Man erhält das Produkt 3 als ein leicht gelbliches Öl (5.2 g, $0.014 \mathrm{~mol}, 14 \%)$.

${ }^{1} \mathrm{H}-\mathrm{NMR}\left(300 \mathrm{MHz}, \mathrm{CDCl}_{3}\right), \delta(\mathrm{ppm}): 0.8$ (t, 2H, $\left.\mathrm{CH}_{2}-\mathrm{Si}\right), 1.8$ (q, 2H, $\mathrm{CH}_{2}$ ), $3.4\left(\mathrm{t}, 2 \mathrm{H}, \mathrm{CH}_{2}-\mathrm{S}\right), 3.6\left(\mathrm{~s}, 9 \mathrm{H}, 3 \times \mathrm{CH}_{3}\right), 4.6\left(\mathrm{~s}, 2 \mathrm{H}, \mathrm{S}-\mathrm{CH}_{2}-\mathrm{Ph}\right), 7.2-7.4(\mathrm{~m}, 5 \mathrm{H}$, $\left.\mathrm{C}_{6} H_{5}\right)$.

${ }^{13} \mathrm{C}-\mathrm{NMR}\left(300 \mathrm{MHz}, \mathrm{CDCl}_{3}\right), \delta(\mathrm{ppm}): 8.7\left(\mathrm{CH}_{2}-\mathrm{Si}\right), 21.6\left(\mathrm{CH}_{2}\right), 39.3\left(\mathrm{CH}_{2}-\mathrm{S}\right)$, $41.3\left(\mathrm{~S}-C \mathrm{H}_{2}-\mathrm{Ph}\right), 50.5\left(\mathrm{O}-C \mathrm{H}_{3}\right), 127.6,128,6,129,1,134.9\left(C_{6} \mathrm{H}_{5}\right), 223.4(C(=\mathrm{S}) \mathrm{S})$.

Elementaranalyse (\%): Kohlenstoff (45.8; ber. 46.4), Wasserstoff (6.1; ber. 6.1), Schwefel (28.3; ber. 26.5).

\section{3-(Methyldimethoxysilan)propyl-(phenylmethyl)-trithiocarbonat 4}

Die synthetische Prozedur von 4 ist identisch mit der von 1, außer dass anstatt von Propanthiol 3-Mercaptopropylmethyldimethoxysilan (18.0 g, $0.10 \mathrm{~mol}$ ) eingesetzt wurde. Das Produkt wurde mit Hilfe der Säulenchromatographie (Silicagel, Pentan : Dichlormethan $\left.=9: 1, R_{\mathrm{f}}=0.52\right)$ gereinigt. Man erhält das Produkt 4 als ein leicht gelbliches Öl (6.2 g, $0.018 \mathrm{~mol}, 18 \%)$.

${ }^{1} \mathrm{H}-\mathrm{NMR}\left(300 \mathrm{MHz}, \mathrm{CDCl}_{3}\right.$ ), $\delta(\mathrm{ppm}): 0.4\left(\mathrm{~s}, 3 \mathrm{H}, \mathrm{CH}_{3}-\mathrm{Si}\right), 0.7$ (t, 2H, $\mathrm{CH}_{2}-\mathrm{Si}$ ), $1.8\left(\mathrm{q}, 2 \mathrm{H}, \mathrm{CH}_{2}\right), 3.4$ (t, 2H, $\left.\mathrm{CH}_{2}-\mathrm{S}\right), 3.6\left(\mathrm{~s}, 6 \mathrm{H}, 2 \times \mathrm{CH}_{3}\right), 4.6\left(\mathrm{~s}, 2 \mathrm{H}, \mathrm{S}-\mathrm{CH}_{2}-\mathrm{Ph}\right)$, 7.2-7.4 (m, 5H, $\left.\mathrm{C}_{6} H_{5}\right)$.

${ }^{13} \mathrm{C}-\mathrm{NMR}\left(300 \mathrm{MHz}, \mathrm{CDCl}_{3}\right), \delta(\mathrm{ppm}): 4.1\left(\mathrm{CH}_{3}-\mathrm{Si}\right), 6.7\left(\mathrm{CH}_{2}-\mathrm{Si}\right), 20.4\left(\mathrm{CH}_{2}\right)$, $38.2\left(\mathrm{CH}_{2}-\mathrm{S}\right), 41.1\left(\mathrm{~S}-\mathrm{CH}_{2}-\mathrm{Ph}\right), 50.2\left(\mathrm{O}-\mathrm{CH}_{3}\right), 127.2,128,1,128.7,133.7\left(C_{6} \mathrm{H}_{5}\right)$, $223.3(C(=\mathrm{S}) \mathrm{S})$.

\section{Propyl-((trimethoxysilan)ethyl-phenylmethyl)-trithiocarbonat $\mathbf{5}$}

Die synthetische Prozedur von $\mathbf{5}$ ist identisch mit der von $\mathbf{1}$, außer dass anstatt von Benzylchlorid ((Chlormethyl)phenylethyl)-trimethoxysilan (27.5 g, $0.10 \mathrm{~mol}$, 
Gemisch aus $m, p$-Isomere) eingesetzt wurde. Das Produkt wurde mit Hilfe der Säulenchromatographie (Silicagel, Pentan : Dichlormethan $=9: 1, R_{\mathrm{f}}=0.42$ ) gereinigt. Man erhält das Produkt 5 als ein leicht gelbliches Öl $(7.4$ g, 0.019 mol, $19 \%)$.

${ }^{1} \mathrm{H}-\mathrm{NMR}\left(300 \mathrm{MHz}, \mathrm{CDCl}_{3}\right), \delta(\mathrm{ppm}): 0.9$ (t, 2H, $\left.\mathrm{CH}_{2}-\mathrm{Si}\right), 1.0\left(\mathrm{t}, 3 \mathrm{H}, \mathrm{CH}_{3}\right), 1.8$ $\left(\mathrm{m}, 2 \mathrm{H}, \mathrm{CH}_{2}\right), 2.7\left(\mathrm{~m}, 2 \mathrm{H}, \mathrm{CH}_{2}-\mathrm{Ph}\right), 3.4\left(\mathrm{t}, 2 \mathrm{H}, \mathrm{CH}_{2}-\mathrm{S}\right), 3.6\left(\mathrm{~s}, 9 \mathrm{H}, 3 \times \mathrm{CH}_{3}\right), 4.6$ (s, 2H, S- $\left.\mathrm{CH}_{2}-\mathrm{Ph}\right), 7.1-7.3\left(\mathrm{~m}, 4 \mathrm{H}, \mathrm{C}_{6} H_{4}\right)$.

${ }^{13} \mathrm{C}-\mathrm{NMR}\left(300 \mathrm{MHz}, \mathrm{CDCl}_{3}\right), \delta(\mathrm{ppm}): 10.9\left(\mathrm{CH}_{2}-\mathrm{Si}\right), 13.2\left(\mathrm{CH}_{3}\right), 21.3\left(\mathrm{CH}_{2}\right)$, $28.3\left(\mathrm{CH}_{2}-\mathrm{Ph}\right), 38.6\left(\mathrm{CH}_{2}-\mathrm{S}\right), 41.3\left(\mathrm{~S}-\mathrm{CH}_{2}-\mathrm{Ph}\right), 50.4\left(\mathrm{O}-\mathrm{CH}_{3}\right), 126.7,127.3,128.2$, 128.8, 129.3, $135.0\left(C_{6} \mathrm{H}_{4}\right), 223.2(C(=\mathrm{S}) \mathrm{S})$.

Elementaranalyse (\%): Kohlenstoff (50.7; ber. 49.1), Wasserstoff (6.8; ber. 6.7), Schwefel (22.1; ber. 24.6).

\section{Propyl-((methyldimethoxysilan)ethyl-phenylmethyl)-trithiocarbonat 6}

Die synthetische Prozedur von $\mathbf{6}$ ist identisch mit der von 1, außer dass anstatt von Benzylchlorid ((Chlormethyl)phenylethyl)-methyldichlorsilan (26.7 g, 0.10 mol, Gemisch aus $m, p$-Isomere) eingesetzt wurde. Außerdem wurde die Menge des Methanolats auf $54.0 \mathrm{~g}$ (0.30 mol) erhöht, um die Dichlorsilangruppe in die Dimethoxygruppe umzuwandeln. Das Produkt wurde mit Hilfe der Säulenchromatographie (Silicagel, Pentan : Dichlormethan $\left.=9: 1, R_{\mathrm{f}}=0.49\right)$ gereinigt. Man erhält das Produkt 6 als ein leicht gelbliches Öl (7.8 g, $0.021 \mathrm{~mol}, 21 \%)$.

${ }^{1} \mathrm{H}-\mathrm{NMR}\left(300 \mathrm{MHz}, \mathrm{CDCl}_{3}\right), \delta(\mathrm{ppm}): 0.4$ (s, 3H, $\left.\mathrm{CH}_{3}-\mathrm{Si}\right), 0.9$ (t, 2H, $\left.\mathrm{CH}_{2}-\mathrm{Si}\right)$, 1.0 (t, 3H, $\left.\mathrm{CH}_{3}\right), 1.8\left(\mathrm{~m}, 2 \mathrm{H}, \mathrm{CH}_{2}\right), 2.7\left(\mathrm{~m}, 2 \mathrm{H}, \mathrm{CH}_{2}-\mathrm{Ph}\right), 3.4\left(\mathrm{t}, 2 \mathrm{H}, \mathrm{CH}_{2}-\mathrm{S}\right), 3.6$ $\left(\mathrm{s}, 6 \mathrm{H}, 2 \times \mathrm{CH}_{3}\right), 4.6\left(\mathrm{~s}, 2 \mathrm{H}, \mathrm{S}-\mathrm{CH}_{2}-\mathrm{Ph}\right), 7.1-7.3\left(\mathrm{~m}, 4 \mathrm{H}, \mathrm{C}_{6} H_{4}\right)$.

${ }^{13} \mathrm{C}-\mathrm{NMR}\left(300 \mathrm{MHz}, \mathrm{CDCl}_{3}\right), \delta(\mathrm{ppm}): 4.4\left(\mathrm{CH}_{3}-\mathrm{Si}\right), 11.3\left(\mathrm{CH}_{2}-\mathrm{Si}\right), 13.3\left(\mathrm{CH}_{3}\right)$, $21.4\left(\mathrm{CH}_{2}\right), 28.6\left(\mathrm{CH}_{2}-\mathrm{Ph}\right), 38.7\left(\mathrm{CH}_{2}-\mathrm{S}\right), 41.4\left(\mathrm{~S}-\mathrm{CH}_{2}-\mathrm{Ph}\right), 50.6\left(\mathrm{O}-\mathrm{CH}_{3}\right), 126.9$, $127.4,128.3,129.0,129.4,135.2\left(C_{6} \mathrm{H}_{4}\right), 223.3(C(=\mathrm{S}) \mathrm{S})$.

\section{Propyl-((dimethylmethoxysilan)ethyl-phenylmethyl)-trithiocarbonat $\mathbf{7}$}

Die synthetische Prozedur von $\mathbf{7}$ ist identisch mit der von 1, außer dass anstatt von Benzylchlorid ((Chlormethyl)phenylethyl)-dimethylchlorsilan (26.7 g, 0.10 mol, Gemisch aus $m, p$-Isomere) eingesetzt wurde. Außerdem wurde die Menge des Methanolat auf $36.0 \mathrm{~g}$ (0.20 mol) erhöht, um die Chlorsilangruppe in die Methoxygruppe umzuwandeln. Das Produkt wurde mit Hilfe der Säulenchromatographie (Silicagel, 
Pentan : Dichlormethan $\left.=9: 1, R_{\mathrm{f}}=0.52\right)$ gereinigt. Man erhält das Produkt 7 als ein leicht gelbliches Öl (8.8 g, $0.024 \mathrm{~mol}, 24 \%$ ).

${ }^{1} \mathrm{H}-\mathrm{NMR}\left(300 \mathrm{MHz}, \mathrm{CDCl}_{3}\right), \delta(\mathrm{ppm}): 0.3\left(\mathrm{~s}, 6 \mathrm{H}, 2 \times \mathrm{CH}_{3}-\mathrm{Si}\right), 0.8$ (t, 2H, $\mathrm{CH}_{2}{ }^{-}$ $\mathrm{Si}), 1.0\left(\mathrm{t}, 3 \mathrm{H}, \mathrm{CH}_{3}\right), 1.8\left(\mathrm{~m}, 2 \mathrm{H}, \mathrm{CH}_{2}\right), 2.7\left(\mathrm{~m}, 2 \mathrm{H}, \mathrm{CH}_{2}-\mathrm{Ph}\right), 3.4\left(\mathrm{t}, 2 \mathrm{H}, \mathrm{CH}_{2}-\mathrm{S}\right)$, $3.6\left(\mathrm{~s}, 3 \mathrm{H}, \mathrm{CH}_{3}\right), 4.6\left(\mathrm{~s}, 2 \mathrm{H}, \mathrm{S}-\mathrm{CH}_{2}-\mathrm{Ph}\right), 7.1-7.3\left(\mathrm{~m}, 4 \mathrm{H}, \mathrm{C}_{6} H_{4}\right)$.

${ }^{13} \mathrm{C}-\mathrm{NMR}\left(300 \mathrm{MHz}, \mathrm{CDCl}_{3}\right), \delta(\mathrm{ppm}): 3.4\left(\mathrm{CH}_{3}-\mathrm{Si}\right), 11.4\left(\mathrm{CH}_{2}-\mathrm{Si}\right), 13.5\left(\mathrm{CH}_{3}\right)$, $21.3\left(\mathrm{CH}_{2}\right), 28.5\left(\mathrm{CH}_{2}-\mathrm{Ph}\right), 38.7\left(\mathrm{CH}_{2}-\mathrm{S}\right), 41.3\left(\mathrm{~S}-\mathrm{CH}_{2}-\mathrm{Ph}\right), 50.5\left(\mathrm{O}-\mathrm{CH}_{3}\right), 126.8$, $127.5,128.4,129.1,129.2,135.1\left(C_{6} \mathrm{H}_{4}\right), 223.4(C(=\mathrm{S}) \mathrm{S})$.

\section{1,4-Phenylenebis(methylen)-dipropylentrithiocarbonate $\mathbf{8}$}

Die synthetische Prozedur von 8 ist identisch mit der von 1, außer dass anstatt von Benzylchlorid $\alpha, \alpha^{\prime}$-Dichlor- $p$-xylol ( $8.7 \mathrm{~g}, 0.05 \mathrm{~mol}$ ) eingesetzt wurde. Das Produkt 8 wurde mit Hilfe der Säulenchromatographie (Silicagel, Dichlormethan, $R_{\mathrm{f}}=$ 0.67) vorgereinigt und in Heptan als gelblich leuchtender Kristall ausgefällt (3.2 g, $0.0079 \mathrm{~mol}, 16 \%)$.

${ }^{1} \mathrm{H}-\mathrm{NMR}\left(300 \mathrm{MHz}, \mathrm{CDCl}_{3}\right), \delta(\mathrm{ppm}): 1.0\left(\mathrm{t}, 6 \mathrm{H}, 2 \times \mathrm{CH}_{3}\right), 1.7$ (quintett,4H, $\left.2 \times \mathrm{CH}_{2}\right), 3.4\left(\mathrm{t}, 4 \mathrm{H}, 2 \times \mathrm{CH}_{2}-\mathrm{S}\right), 4.6\left(\mathrm{~s}, 4 \mathrm{H}, 2 \times \mathrm{S}-\mathrm{CH}_{2}-\mathrm{Ph}\right), 7.3\left(\mathrm{~s}, 4 \mathrm{H}, \mathrm{C}_{6} H_{4}\right)$.

${ }^{13} \mathrm{C}-\mathrm{NMR}\left(300 \mathrm{MHz}, \mathrm{CDCl}_{3}\right), \delta(\mathrm{ppm}): 13.4\left(\mathrm{CH}_{3}\right), 21.5\left(\mathrm{CH}_{2}\right), 38.8\left(\mathrm{~S}-\mathrm{CH}_{2}\right)$, $40.8\left(\mathrm{~S}-C \mathrm{H}_{2}-\mathrm{Ph}\right), 129.5,134.7\left(C_{6} \mathrm{H}_{4}\right), 223.6(C(=\mathrm{S}) \mathrm{S})$.

\section{1,6-Hexyl-diphenylmethyltrithiocarbonate $\mathbf{9}$}

Die synthetische Prozedur von $\mathbf{9}$ ist identisch mit der von $\mathbf{1}$, außer dass anstatt von Propanthiol 1,6-Hexandithiol (7.5 g, 0.05 mol) eingesetzt wurde. Das Produkt 9 wurde mit Hilfe der Säulenchromatographie (Silicagel, Dichlormethan, $R_{\mathrm{f}}=0.62$ ) vorgereinigt und in Heptan als gelblich leuchtender Kristall ausgefällt (4.4 g, $0.0091 \mathrm{~mol}$, $18 \%)$.

${ }^{1} \mathrm{H}-\mathrm{NMR}\left(300 \mathrm{MHz}, \mathrm{CDCl}_{3}\right), \delta(\mathrm{ppm}): 1.3\left(\mathrm{~b}, 4 \mathrm{H}, 2 \times \mathrm{CH}_{2}\right), 1.7\left(\mathrm{~b}, 4 \mathrm{H}, 2 \times \mathrm{CH}_{2}\right)$, $3.3\left(\mathrm{t}, 4 \mathrm{H}, 2 \times \mathrm{CH}_{2}-\mathrm{S}\right), 4.6\left(\mathrm{~s}, 4 \mathrm{H}, 2 \times \mathrm{S}-\mathrm{CH}_{2}-\mathrm{Ph}\right), 7.3\left(\mathrm{~b}, 10 \mathrm{H}, 2 \times \mathrm{C}_{6} H_{5}\right)$.

${ }^{13} \mathrm{C}-\mathrm{NMR}\left(300 \mathrm{MHz}, \mathrm{CDCl}_{3}\right), \delta(\mathrm{ppm}): 27.7,28.1\left(C \mathrm{H}_{2}\right), 36.6\left(\mathrm{~S}-\mathrm{CH}_{2}\right), 41.2$ $\left(\mathrm{S}-\mathrm{CH}_{2}-\mathrm{Ph}\right), 127.8,128.8,129.3,135.1\left(C_{6} \mathrm{H}_{5}\right), 223.9(C(=\mathrm{S}) \mathrm{S})$.

\section{1,4-Phenylenbis-(methylen)-di(trimethoxysilanpropylentrithiocarbonate) $\mathbf{1 0}$}

Die synthetische Prozedur von 10 ist identisch mit der von 1, außer dass anstatt von Propanthiol 3-Mercaptopropyltrimethoxysilan (19.6 g, $0.10 \mathrm{~mol}$ ) und anstatt von 
Benzylchlorid $\alpha, \alpha^{\prime}$-Dichlor- $p$-xylol (8.7 g, $0.05 \mathrm{~mol}$ ) eingesetzt wurde. Das Produkt wurde mit Hilfe der Säulenchromatographie (Silicagel, Dichlormethan, $R_{\mathrm{f}}=0.58$ ) gereinigt. Man erhält das Produkt 10 als einen gelblichen Feststoff (1.2 g, 0.0018 mol, $3.7 \%)$.

${ }^{1} \mathrm{H}-\mathrm{NMR}\left(300 \mathrm{MHz}, \mathrm{CDCl}_{3}\right), \delta(\mathrm{ppm}): 0.8\left(\mathrm{t}, 4 \mathrm{H}, 2 \times \mathrm{Si}-\mathrm{CH}_{2}\right), 1.8(\mathrm{q}, 4 \mathrm{H}, 2 \times$ $\left.\mathrm{CH}_{2}\right), 3.4\left(\mathrm{t}, 4 \mathrm{H}, 2 \times \mathrm{CH}_{2}-\mathrm{S}\right), 3.6\left(\mathrm{~s}, 18 \mathrm{H}, 6 \times \mathrm{O}-\mathrm{CH}_{3}\right), 4.6\left(\mathrm{~s}, 4 \mathrm{H}, 2 \times \mathrm{S}-\mathrm{CH}_{2}-\mathrm{Ph}\right)$, $7.2\left(\mathrm{~s}, 4 \mathrm{H}, \mathrm{C}_{6} H_{4}\right)$.

${ }^{13} \mathrm{C}-\mathrm{NMR}\left(300 \mathrm{MHz}, \mathrm{CDCl}_{3}\right), \delta(\mathrm{ppm}): 8.7\left(\mathrm{Si}-\mathrm{CH}_{2}\right), 21.6\left(\mathrm{CH}_{2}\right), 39.5\left(\mathrm{~S}-\mathrm{CH}_{2}\right)$, $40.8\left(\mathrm{~S}-\mathrm{CH}_{2}-\mathrm{Ph}\right), 50.5\left(\mathrm{O}-\mathrm{CH}_{3}\right), 129.5,134.6\left(C_{6} \mathrm{H}_{4}\right), 223.3(C(=\mathrm{S}) \mathrm{S})$.

\section{1,6-Hexyl-di((trimethoxysilanethyl-phenylmethyl)-trithiocarbonate) $\mathbf{1 1}$}

Die synthetische Prozedur von 11 ist identisch mit der von 1, außer dass anstatt von Propanthiol 1,6-Hexandithiol (7.5 g, $0.05 \mathrm{~mol}$ ) und anstatt von Benzylchlorid ((Chlormethyl)phenylethyl)-trimethoxysilan (27.5 g, $0.10 \mathrm{~mol}$, Gemisch aus m,pIsomere)) eingesetzt wurde. Das Produkt 11 wurde mit Hilfe der Säulenchromatographie (Silicagel, Dichlormethan, $R_{\mathrm{f}}=0.57$ ) vorgereinigt und in Heptan als gelblich leuchtender Kristall ausgefällt (2.4 g, $0.0031 \mathrm{~mol}, 6.2 \%)$.

${ }^{1} \mathrm{H}-\mathrm{NMR}\left(300 \mathrm{MHz}, \mathrm{CDCl}_{3}\right), \delta(\mathrm{ppm}): 0.9$ (t, $\left.4 \mathrm{H}, 2 \times \mathrm{CH}_{2}-\mathrm{Si}\right), 1.0(\mathrm{t}, 4 \mathrm{H}, 2 \times$ $\left.\mathrm{CH}_{2}\right), 1.8\left(\mathrm{~m}, 4 \mathrm{H}, 2 \times \mathrm{CH}_{2}\right), 2.7\left(\mathrm{~m}, 4 \mathrm{H}, 2 \times \mathrm{CH}_{2}-\mathrm{Ph}\right), 3.4\left(\mathrm{t}, 4 \mathrm{H}, 2 \times \mathrm{CH}_{2}-\mathrm{S}\right), 3.6$ $\left(\mathrm{s}, 18 \mathrm{H}, 6 \times \mathrm{CH}_{3}\right), 4.6\left(\mathrm{~s}, 4 \mathrm{H}, 2 \times \mathrm{S}-\mathrm{CH}_{2}-\mathrm{Ph}\right), 7.1-7.3\left(\mathrm{~m}, 8 \mathrm{H}, 2 \times \mathrm{C}_{6} H_{4}\right)$.

${ }^{13} \mathrm{C}-\mathrm{NMR}\left(300 \mathrm{MHz}, \mathrm{CDCl}_{3}\right), \delta(\mathrm{ppm}): 10.8\left(\mathrm{CH}_{2}-\mathrm{Si}\right), 27.5\left(\mathrm{CH}_{2}\right), 28.1\left(\mathrm{CH}_{2}\right)$, $28.6\left(\mathrm{CH}_{2}-\mathrm{Ph}\right), 37.6\left(\mathrm{CH}_{2}-\mathrm{S}\right), 41.2\left(\mathrm{~S}-\mathrm{CH}_{2}-\mathrm{Ph}\right), 50.4\left(\mathrm{O}-\mathrm{CH}_{3}\right), 126.5,127.1,128.4$, 128.7, 129.6, $135.2\left(C_{6} \mathrm{H}_{4}\right), 223.5(C(=\mathrm{S}) \mathrm{S})$.

\subsubsection{Polymerisation}

Das Monomer (Methylacrylat MA, Methylmethacrylat MMA bzw. Styrol Sty) wurde mittels dreimaligem „freeze-pump-and-thaw" an einer Hochvakuumpumpe (Edwards, EXC 120) entgast. Die Lösung wurde in eine mit Argon befüllte Handschuhbox (Braun, Lab Master 130) eingeschleust und darin mit AIBN als Initiator und einem entsprechenden Trithiocarbonat als RAFT-Agens vermischt. Die Lösung wurde dann in Schraubdeckelgläser aufgeteilt, die anschließend mit den entsprechenden gasdichten Deckeln in der Handschuhbox verschlossen wurden.

Die Polymerisation wurde bei Umgebungsdruck in einem Thermoblock (Rotilabo, 
Block Heater H 250) bei $60{ }^{\circ} \mathrm{C}$ durchgeführt. Nach den angegebenen Zeiten wurden die Schraubdeckelgläser aus dem Thermoblock entfernt und zum Reaktionsabbruch in ein Eisbad gestellt.

Zur gravimetrischen Monomerumsatzbestimmung wurde nach erfolgter Polymerisation das Monomer bei Zimmertemperatur unter vermindertem Druck entfernt. Die Molmassenverteilung des toten Polymers wurde mittels Gelpermeationschromatographie (GPC) bestimmt.

\subsubsection{Blockcopolymerisation}

Das Makro-RAFT-Agens wurde in Methanol ausgefällt, um niedermolekulare Verunreinigungen zu entfernen. Nach Trocknen unter vermindertem Druck wurde das Makro-RAFT-Agens in die Handschuhbox geschleust. Eine Comonomerlösung wurde mittels dreimaligem "freeze-pump-and-thaw" entgast und in der Handschuhbox mit dem Makro-RAFT-Agens und AIBN vermischt. Die Polymerisationslösung wurde dann in Schraubdeckelgläser aufgeteilt. Der weitere Verlauf der Polymerisation und der Charakterisierung verliefen analog zu der oben beschriebenen Polymerisation.

\subsubsection{Kondensation}

Zuerst wurde eine gesättigte Lösung des Trimethoxysilan-haltigen Polymers in Toluol hergestellt. Nach dem Hinzufügen einer katalytischen Menge Wasser wurde ein Stickstoffstrom durch die Mischung geleitet, um Sauerstoff zu entfernen. Die Mischung wurde bei Zimmertemperatur gerührt. Nach einer bestimmten Zeit wurde die Molmassenverteilung des erhaltenen Polymers mittels GPC bestimmt.

\subsection{Ergebnisse und Diskussion}

\subsubsection{Homopolymer}

In diesem Abschnitt werden die RAFT-Polymerisationen von Styrol, Methylacrylat bzw. Methylmethacrylat mit dem RAFT-Agens Propyl(phenylmethyl)trithiocarbonat $(\mathrm{PPT}, \mathbf{1})$ beschrieben. Die Ergebnisse sind jedoch generell auch auf andere in Abbildung 7.1 und 7.2 gezeigte Trithiocarbonate übertragbar. 


\section{RAFT-Polymerisation von Styrol}

Die RAFT-Polymerisation von Styrol mit PPT ist in Abbildung 7.5 dargestellt. PPT ist als RAFT-Agens für die kontrollierte Polymerisation von Styrol mäßig gut geeignet. Es zeigt bei Styrol-Polymerisationen keinen Induktions- und keinen Retardierungseffekt. Die mittlere Molmasse des Polystyrols nimmt mit dem Monomerumsatz zu und stimmt mit der berechneten theoretischen Molmassen $M_{\mathrm{n}}^{\text {theo }}$ gut überein.

$$
M_{\mathrm{n}}^{\text {theo }}=\frac{[\mathrm{M}]_{0} \cdot M W_{\text {Monomer }} \cdot U_{\mathrm{M}}}{[\mathrm{RAFT}]_{0}+d \cdot f \cdot\left([\mathrm{I}]_{0}-[\mathrm{I}]_{U}\right)}
$$

In dieser Gleichung ist $M_{\mathrm{n}}^{\text {theo }}$ die theoretische Molmasse, $M W_{\text {Monomer }}$ die Molmasse des Monomers, $[\mathrm{RAFT}]_{0}$ die Startkonzentration des RAFT-Agens, $d$ spiegelt den Terminierungsmodus wider ${ }^{1}, f$ ist die Initiatoreffektivität, $[\mathrm{I}]_{0}$ die Startkonzentration des Initiators und $[\mathrm{I}]_{U}$ die Initiatorkonzentratration beim Monomerumsatz $U$.

Die Polydispersität ist für eine gut kontrollierte Polymerisation relativ groß in der Anfangsphase, bei hohem Monomerumsatz wird sie allerdings deutlich kleiner als 1.5. Wegen der für die Styrol-Polymerisation schlechten Abgangsgruppe läuft das Vorgleichgewicht des RAFT-Mechanismus relativ langsam ab. Daher zeigt die RAFT-Polymerisation von Styrol mit 1 ein Hybridverhalten, das sich durch einen sprunghaften Anstieg der Molmasse in der Anfangsphase, gefolgt von einer kontrollierten Polymerisation zeichnet. Das Hybridverhalten verursacht eine Verbreiterung der Molmassenverteilung des gebildeten Polystyrols, besonders in der Anfangsphase verläuft die Polymerisation weitgehend unkontrolliert. Das Hybridverhalten kann durch Variation der Abgangsgruppe unterdrückt werden, es ist jedoch weniger bedeutend bei hohem Monomerumsatz.

\section{RAFT-Polymerisation von Methylacrylat}

Für die Polymerisation von Methylacrylat ist PPT ein sehr gutes RAFT-Agens, wie man in der Abbildung 7.6 sehen kann. Die mittlere Molmasse nimmt linear mit dem Monomer-Umsatz zu. Die nach unten gerichtete Abweichung im Vergleich zu der theoretischen Molmasse $M_{\mathrm{n}}^{\text {theo }}$ weist auf eine Transferreaktion der sehr reaktiven Methylacrylat-Radikale bei höherem Monomerumsatz hin.

\footnotetext{
${ }^{1} d$ ist die Anzahl der Polymerkette, die beim Terminierungsprozess gebildet wird: $d=1$ für Terminierung durch Kombination und $d=2$ für Terminierung durch Disproportionierung.
} 


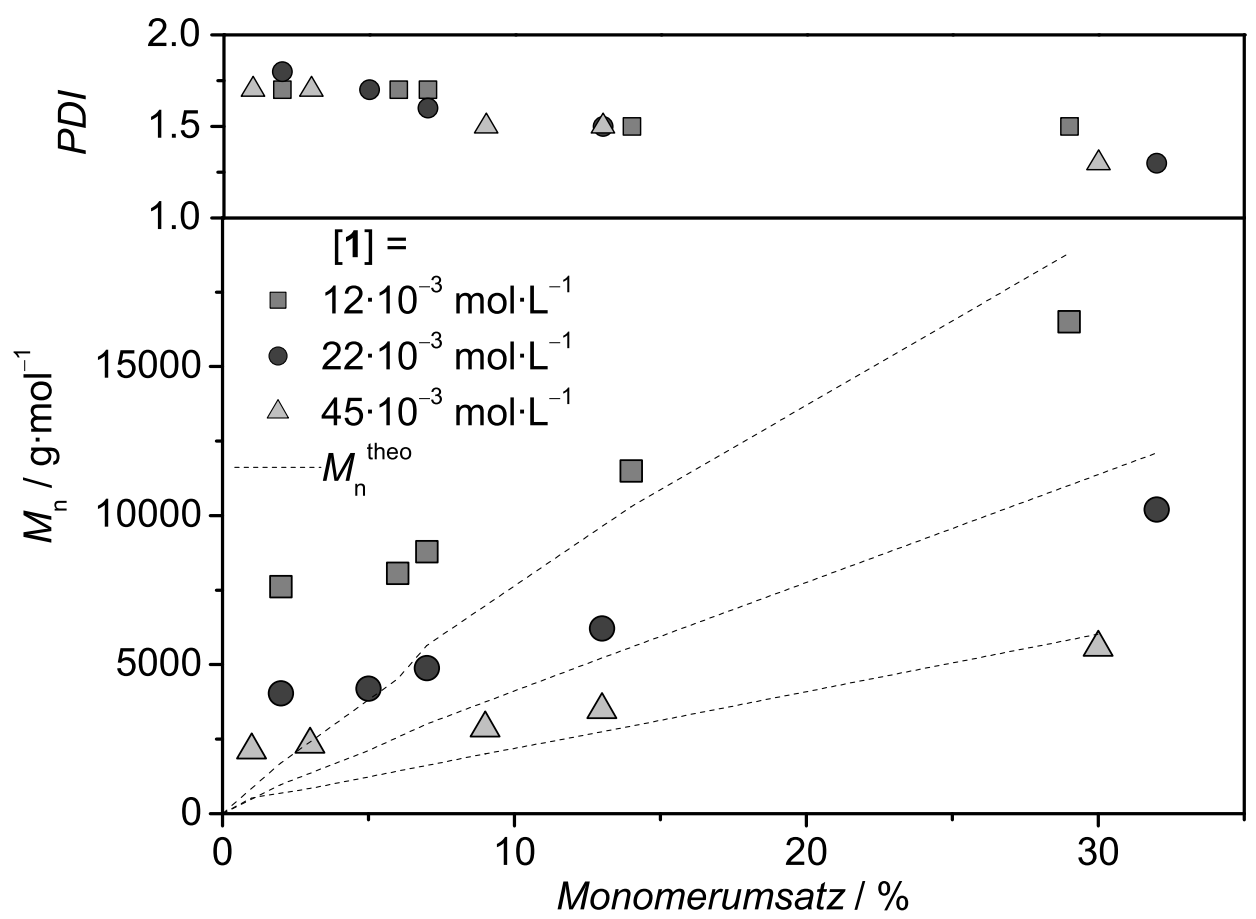

Abbildung 7.5: Auftragung des Zahlenmittels der Molmassen $M_{\mathrm{n}}$ und Polydispersität PDI gegen den Monomerumsatz für die RAFT-Polymerisation von Styrol bei $60{ }^{\circ} \mathrm{C}$ mit verschiedenen Konzentrationen von 1 und $4.5 \cdot 10^{-3} \mathrm{~mol} \cdot \mathrm{L}^{-1}$ AIBN. Die gepunkteten Linien stellen die theoretischen Molmasse $M_{\mathrm{n}}^{\text {theo }}$ dar.

Das aus der RAFT-Polymerisation erhaltene Polymethylacrylat hat eine Polydispersität kleiner als 1.1, d. h. eine extrem enge Molmassenverteilung. Die kleine Polydispersität bleibt bis zu vollständigem Monomerumsatz weitgehend unverändert.

Beim kinetischen Vergleich mit einer konventionellen radikalischen Polymerisation von Methylacrylat hat die RAFT-Polymerisation mit PPT eine etwas geringere Propagierungsgeschwindigkeit, die mit steigender Konzentration des RAFT-Agens abnimmt. Der Retardierungseffekt ist bei der Handhabung der Experimente sogar von Vorteil, da die Polymerisationsgeschwindigkeit von Methylacrylat ansonsten sehr hoch ist. 


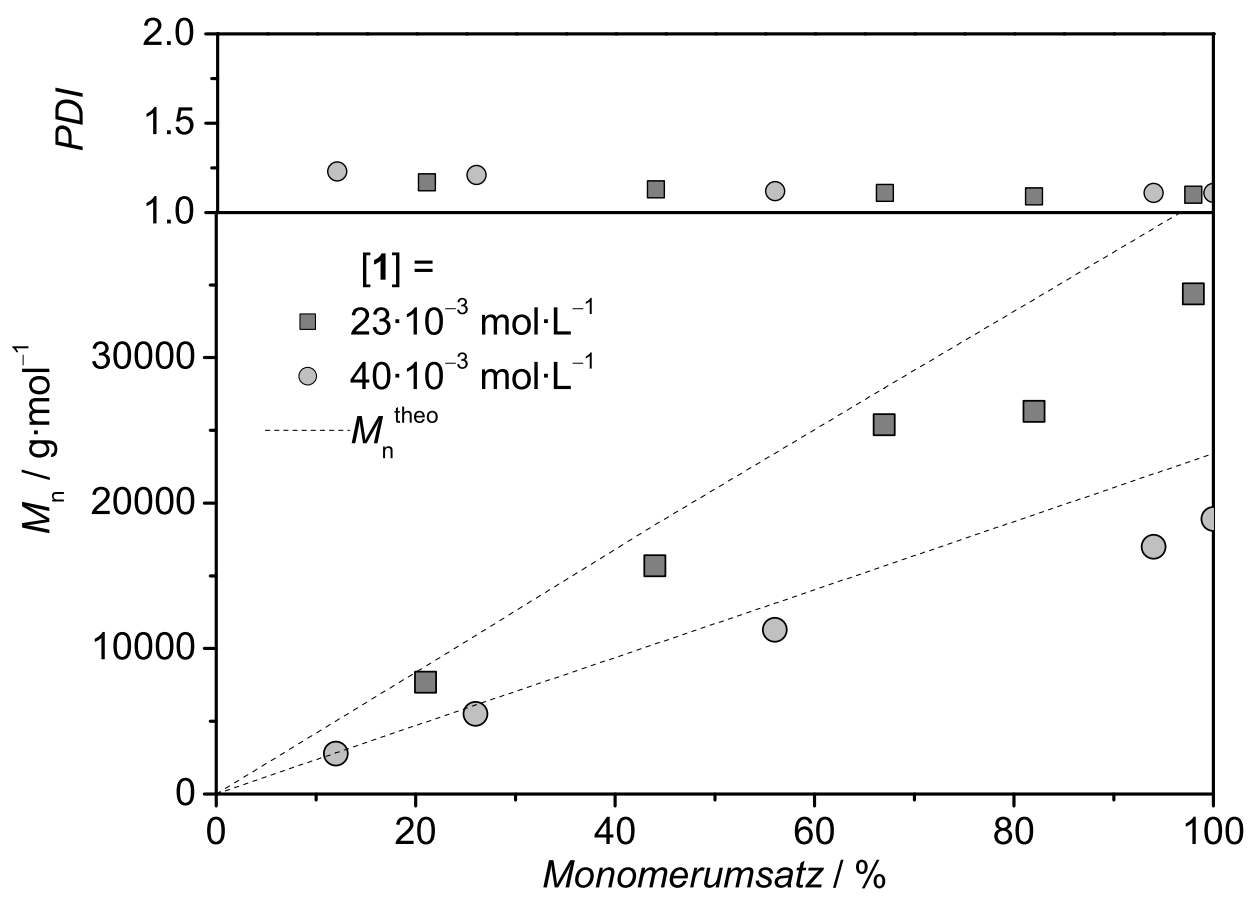

Abbildung 7.6: Auftragung des Zahlenmittels der Molmassen $M_{\mathrm{n}}$ und Polydispersität PDI gegen den Monomerumsatz für die RAFT-Polymerisation von Methylacrylat bei $60{ }^{\circ} \mathrm{C}$ mit verschiedenen Konzentrationen von 1 und $4.7 \cdot 10^{-3} \mathrm{~mol} \cdot \mathrm{L}^{-1}$ AIBN. Die gepunkteten Linien stellen die theoretischen Molmasse $M_{\mathrm{n}}^{\text {theo }}$ dar.

\section{RAFT-Polymerisation von Methylmethacrylat}

Das RAFT-Agens PPT hat überhaupt keinen erkennbaren Einfluss auf die MMAPolymerisation, wie man in der Abbildung 7.7 erkennen kann. Die mittlere Molmasse bleibt konstant über einen weiten Bereich des Monomerumsatzes und bei verschiedenen Konzentrationen von PPT. Außerdem ist sie beim Vergleich mit der theoretischen Molmasse sehr hoch. Die Polydispersität ist sehr groß und beträgt zwischen 2.5 und 3.0. Das System zeigt kein Hybridverhalten, keinen Induktions- und Retardierungseffekt. Dies weist darauf hin, dass es sich um eine konventionelle radikalische Polymerisation von MMA ohne jeglichen Einfluss von PPT handelt.

Der Grund für die fehlende Kontrolle ist, dass PPT eine schlechte Abgangsgruppe für MMA-Polymerisation hat. Das Benzylradikal ist weniger stabil als ein MMA-Radikal. Außerdem ist im intermediären RAFT-Radikal die chemische Bin- 


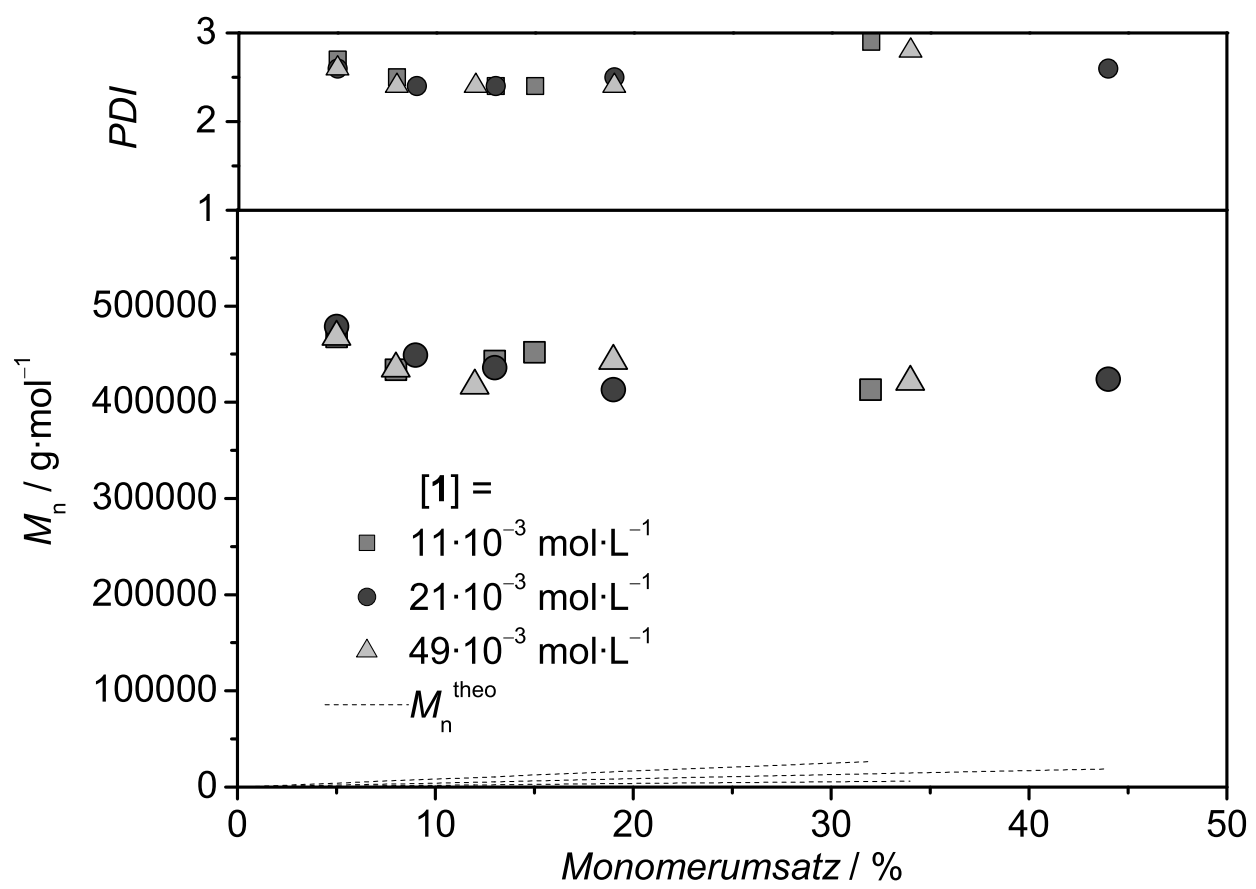

Abbildung 7.7: Auftragung des Zahlenmittels der Molmassen $M_{\mathrm{n}}$ und Polydispersität PDI gegen den Monomerumsatz für die RAFT-Polymerisation von MMA bei $60{ }^{\circ} \mathrm{C}$ mit verschiedenen Konzentrationen von 1 und $4.3 \cdot 10^{-3} \mathrm{~mol} \cdot \mathrm{L}^{-1}$ AIBN. Die gepunkteten Linien stellen die theoretischen Molmasse $M_{\mathrm{n}}^{\text {theo }}$ dar.

dung zwischen dem Schwefelatom und der Benzylgruppe stabiler als die Bindung zwischen dem Schwefelatom und der MMA-Gruppe. Das Vorgleichgewicht des RAFTMechanismus wird dadurch behindert und kann nicht ablaufen. Für eine erfolgreiche RAFT-Polymerisation von MMA müsste das Trithiocarbonat eine andere, weniger stabile Abgangsgruppe besitzen. Mögliche Abgangsgruppen für die MMAPolymerisation werden in Abbildung 2.13 in Kapitel 2 gezeigt.

Andere Strategien, MMA-Einheiten in die Polymerkette ohne Änderung der schlechten Abgangsgruppe des RAFT-Agens einzubauen, werden u. a. im nächsten Abschnitt vorgestellt. 


\subsubsection{Copolymer}

Bei einer Copolymerisation werden mehrere strukturell uneinheitliche Monomere als Comonomere in eine Polymerkette eingebaut. Die entstehenden Copolymere enthalten definitionsgemäß zwei oder mehr unterschiedliche Struktureinheiten. Die Anordnung der Comonomere, z. B. der von zwei Monomeren A und B, in die wachsende Copolymerkette kann unterschiedlich erfolgen und zu

- statistischen -ABAABABBB-,

- alternierenden -ABABABAB-,

- oder Block-Copolymeren -AAABBBAAA-

führen. Über die Wahl von Art und Menge der bei der Copolymerisation eingesetzten Monomere und deren Anordnung im Copolymere lassen sich die PolymerEigenschaften gezielt beeinflussen.

\section{Blockcopolymer aus einem Makro-RAFT-Agens}

Die Herstellung des Blockcopolymers aus Styrol bzw. Methylacrylat gestaltet sich relativ einfach. Dabei ist es gleichgültig, ob das Styrol oder das Methylacrylat zuerst polymerisiert wird ${ }^{2}$. Das erhaltene Blockcopolymer hat eine kontrollierte Molmasse und eine kleine Polydispersität unterhalb von 1.2.

Die Synthese eines zweiten MMA-Blocks aus einem Makro-RAFT-Agens, das aus der RAFT-Polymerisation von Styrol bzw. Methylacrylat stammt, ist nicht geglückt. Abbildung 7.8 zeigt die Molmassenverteilung des erhaltenen polymeren Produktes.

Die polymere Mischung enthält zwei Fraktionen. Die Fraktion mit der kleineren Molmasse ist das eingesetzte Makro-RAFT-Agens, dessen Trithiocarbonatgruppe mit dem UV/vis-Detektor nachgewiesen wird. Die andere Fraktion ist UV-inaktiv und gehört zu Polymethylmethacrylat, das aus der konventionellen radikalischen Polymerisation entstanden ist. Auch hier ist die schlechte Abgangsgruppe, die das Polystyrol bzw. das Polymethylacrylat darstellt, für die unkontrollierte Polymerisation von MMA verantwortlich.

\footnotetext{
${ }^{2}$ RAFT ist eine hervorragende Methode zur Herstellung von Blockcopolymer. Es muss jedoch bei der Herstellung beachtet werden, welcher Block zuerst synthetisiert wird. Das Monomer, das als Radikal stabiler ist, muss zuerst polymerisiert werden.
} 


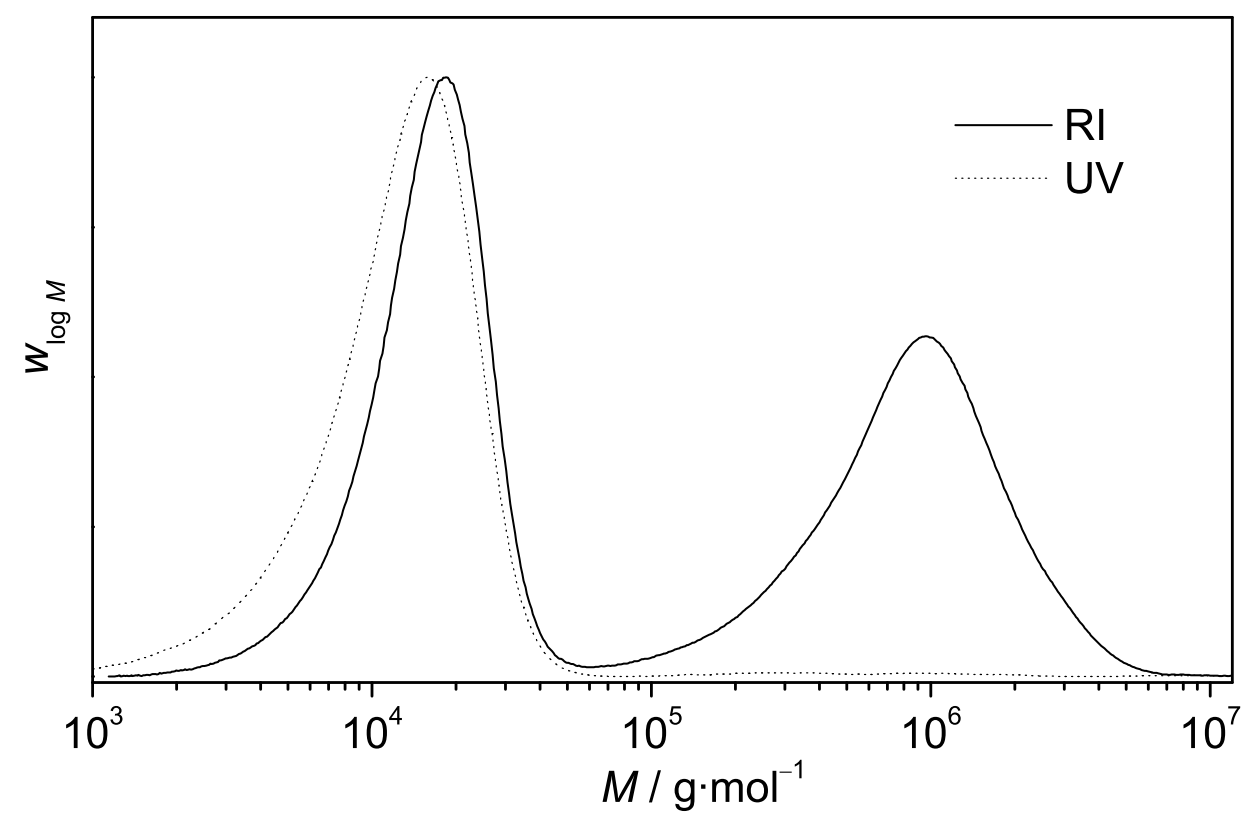

Abbildung 7.8: Molmassenverteilung des polymeren Produktes, die aus dem RI-Signal und dem umgewandelten UV-Signal $(\lambda=310 \mathrm{~nm})$ stammen, gegen der Molmasse auf logarithmischer Skala. Das polymere Produkt wurde aus einer Reaktion von MMA mit dem Makro-RAFT-Agens $\left(4.3 \cdot 10^{-3} \mathrm{~mol} \cdot \mathrm{L}^{-1}\right.$, Polystyrol, $\left.M_{\mathrm{n}}=12500 \mathrm{~g} / \mathrm{mol}, P D I=1.3\right)$ und $\operatorname{AIBN}\left(1.0 \cdot 10^{-3} \mathrm{~mol} \cdot \mathrm{L}^{-1}\right)$ bei $60{ }^{\circ} \mathrm{C}$ nach einer Reaktionszeit von eine Stunde gebildet.

\section{Statistisches Copolymer}

Das System Styrol/MMA hat die Copolymerisationsparameter von $r_{1}=0.52$ und $r_{2}=0.46$ [150]. Es eignet sich deshalb gut für die Herstellung von statistischen Copolymerketten über einen weiten Umsatzbereich. Während die Polymerisation von MMA, wie oben bereits beschrieben, mit PPT bzw. Makro-RAFT-Agens nicht kontrolliert verläuft, bietet das Styrol/MMA-System eine Möglichkeit, MMA-Einheiten in die Polymerkette während der kontrollierten RAFT-Polymerisation von Styrol einzubauen.

Die Copolymerisation von MMA/Styrol-Gemischen mit PPT 1 verläuft kontrolliert, auch wenn die Polydispersitäten etwas größer als 1.5 sind (siehe Abbildung 7.9). Die mittlere Molmasse steigt mit dem Monomerumsatz und stimmt mit der berechneten theoretischen Molmasse überein. Das UV-Signal des Copolymers 


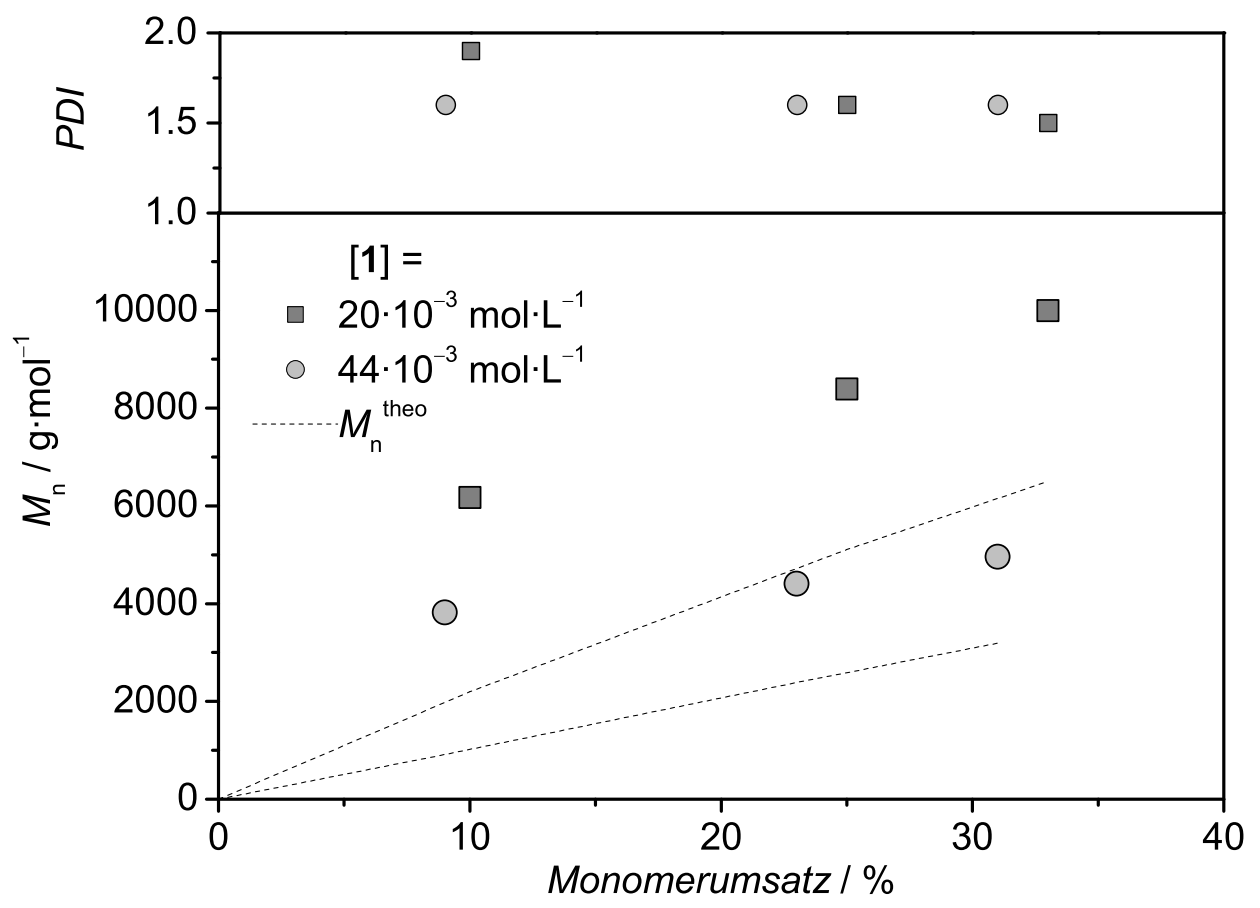

Abbildung 7.9: Auftragung des Zahlenmittels der Molmassen $M_{\mathrm{n}}$ und Polydispersität PDI gegen den Monomerumsatz für die RAFT-Copolymerisation von MMA und Styrol (Massenverhältnis $m_{\mathrm{MMA}}: m_{\mathrm{Sty}}=30: 70$ ) bei $60{ }^{\circ} \mathrm{C}$ mit verschiedenen Konzentrationen von 1 und $5 \cdot 0 \cdot 10^{-3} \mathrm{~mol} \cdot \mathrm{L}^{-1}$ AIBN. Die gepunkteten Linien stellen die theoretischen Molmassen $M_{\mathrm{n}}^{\text {theo }}$ dar.

aus den GPC-Messungen bewies, dass jede Polymerkette eine Trithiocarbonatgruppe enthält.

Die gute Kontrolle des Styrol/MMA-Systems kann bis zu einem MMA-Massenanteil von $30 \%$ gehalten werden. Wenn der MMA-Massenanteil auf $50 \%$ erhöht wird, bleibt die Molmasse über einen weiten Bereich des Monomerumsatzes konstant. Außerdem ist sie im Vergleich mit der theoretischen Molmasse sehr groß.

\section{Blockcopolymer über einmalige Transferreaktion}

Das RAFT-Agens 2 enthält außer der Trithiocarbonatgruppe eine Thiolgruppe, die eine vielseitige Funktionalität für organische Chemie darstellt. Thiolverbindungen spielen in der Natur eine wichtige Rolle wie z. B. bei der Proteinstruktur und der 
Signaltransduktion $[151,152]$. Außerdem haben Thiolverbindungen eine starke Affinität zu Metallsubstraten wie Gold oder Silber. Deshalb werden thiolfunktionalisierte Polymere oft dazu verwendet, um Gold-Nanopartikel zu stabilisieren [153, 154]. Das RAFT-Agens 2 könnte deshalb für die Untersuchung der heterogenen RAFTPolymerisation an Goldoberflächen eingesetzt werden.

In dieser Arbeit wurde das RAFT-Agens 2 verwendet, um Blockcopolymere mit einem MMA-Block zu synthetisieren, was mit PPT 1 nicht geglückt war. Dazu wurde zuerst die radikalische Polymerisation von MMA mit 2 durchgeführt. Die Trithiocarbonatgruppe hatte, wie oben bereits beschrieben, keinen Einfluss auf die MMAPolymerisation. Das RAFT-Agens 2 fungierte hier also nicht als ein kontrollierendes RAFT-Agens, sondern über die Thiolgruppe als Kettenüberträger bzw. als Regler. Abbildung 7.10 stellt den Verlauf der neuen semi-kontrollierten Polymerisationsmethode bildlich dar.

Das Produkt aus der MMA-Polymerisation enthielt das Polymethylmethacrylat (PMMA) und das restliche RAFT-Agens 2 als Verunreinigung. Das Polymer, das entsprechend der konventionellen radikalischen Polymerisation eine breite Molmassenverteilung hat, enthielt eine Trithiocarbonatgruppe, die mittels UV-Detektor des GPC-Experiments nachgewiesen wurde. Der Anteil des toten Polymers wurde durch die Verwendung eines sehr kleinen Initiator-Transferagens-Verhältnisses minimiert. Die niedermolekulare Verunreinigung wurde durch Ausfällen in Methanol vollständig aus der polymeren Masse entfernt. Da das PMMA mit einer Molmasse von bis zu $10 \mathrm{~kg} / \mathrm{mol}$ in Methanol etwas löslich ist, ging ein Teil von PMMA durch das Ausfällen verloren, was die Molmassenverteilung des erhaltenen PMMA enger werden ließ (siehe Abbildung 7.10).

Anschließend wurde das erhaltene PMMA als Makro-RAFT-Agens in der Polymerisation von Styrol bzw. Methylacrylat eingesetzt. Das entstehende Blockcopolymer besitzt eine enge unimodale Molmassenverteilung und enthält eine Trithiocarbonatgruppe, die sich zwischen beiden Polymerblöcken befindet. Die Ergebnisse der Polymerisationen von Methylacrylat mit dem anfallenden Makro-RAFT-Agens sind in Abbildung 7.11 dargestellt.

Die Molmassen-Monomerumsatz-Auftragung in Abbildung 7.11 zeigt eine sehr gute Kontrolle des Makro-RAFT-Agens in der MA-Polymerisation. Die mittlere 


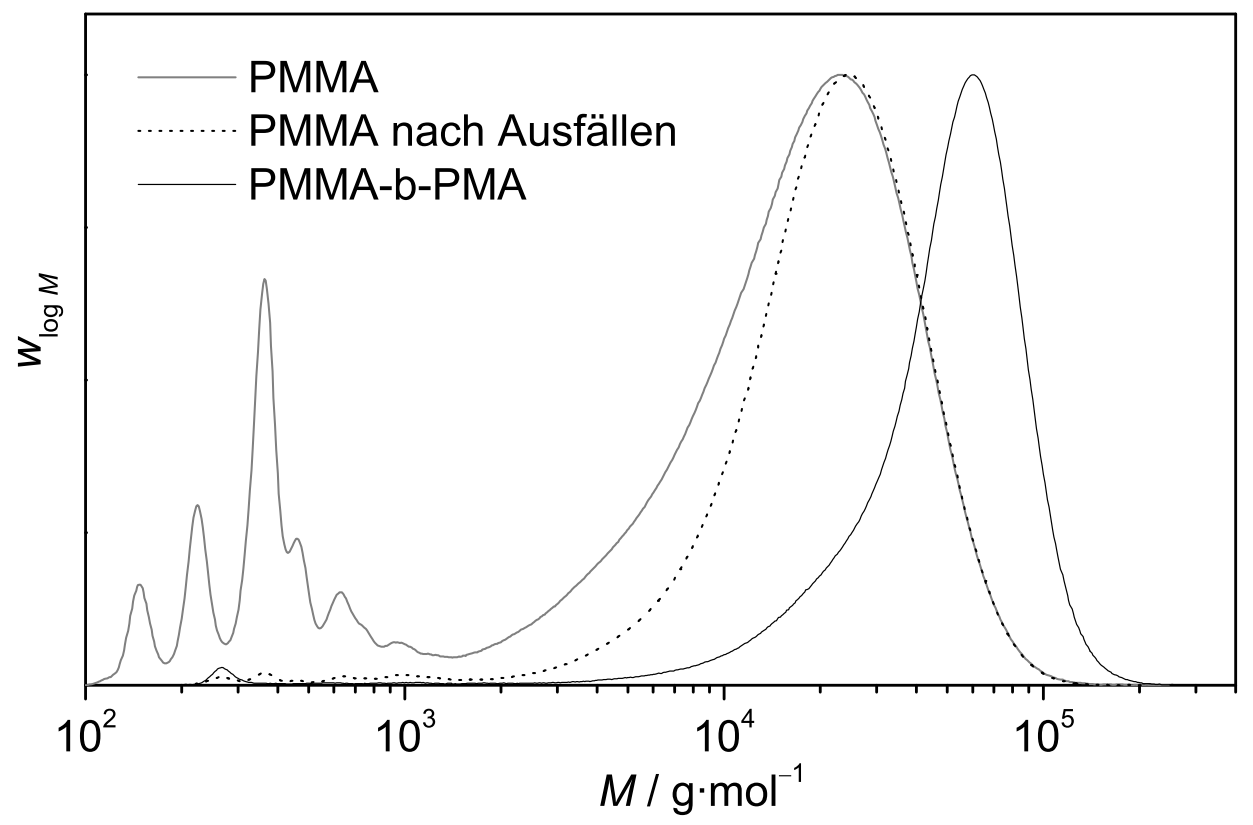

Abbildung 7.10: Molmassenverteilung des polymeren Produktes gegen die Molmasse in logarithmischer Skala. Das Polymethylmethacrylat PMMA wurde aus einer Reaktion von MMA mit $2\left(127 \cdot 10^{-3} \mathrm{~mol} \cdot \mathrm{L}^{-1}\right)$ und AIBN $\left(0.4 \cdot 10^{-3} \mathrm{~mol} \cdot \mathrm{L}^{-1}\right)$ bei $60{ }^{\circ} \mathrm{C}$ nach einer Reaktionszeit von 70 Stunden gebildet. Nach dem Ausfällen des PMMA in Methanol wurde die Polymerisation von MA mit dem erhaltenen Makro-RAFT-Agens $\left(7.5 \cdot 10^{-3} \mathrm{~mol} \cdot \mathrm{L}^{-1}\right)$ durchgeführt.

Molmasse steigt linear mit dem Monomerumsatz an und zeigt eine gute Übereinstimmung mit dem berechneten theoretischen Wert. Auch die Polydispersität mit den Werten zwischen 1.4 und 1.6 ist für diese semi-kontrollierte Polymerisationsmethode relativ niedrig.

\section{Triblockcopolymer}

Im Prinzip ist das mittels RAFT-Technik hergestellte Diblockcopolymer auch wieder ein Makro-RAFT-Agens, mit dem man einen neuen dritten Block aufbauen kann. Doch sowohl Reinheit als auch die Qualität des Blockpolymers nehmen mit der Anzahl der Polyreaktionen stark ab, während der experimentelle Aufwand sich erhöht. Eine Variation der RAFT-Polymerisation, die diese Nachteile minimiert, ist die Verwendung eines bifunktionellen RAFT-Agens. 


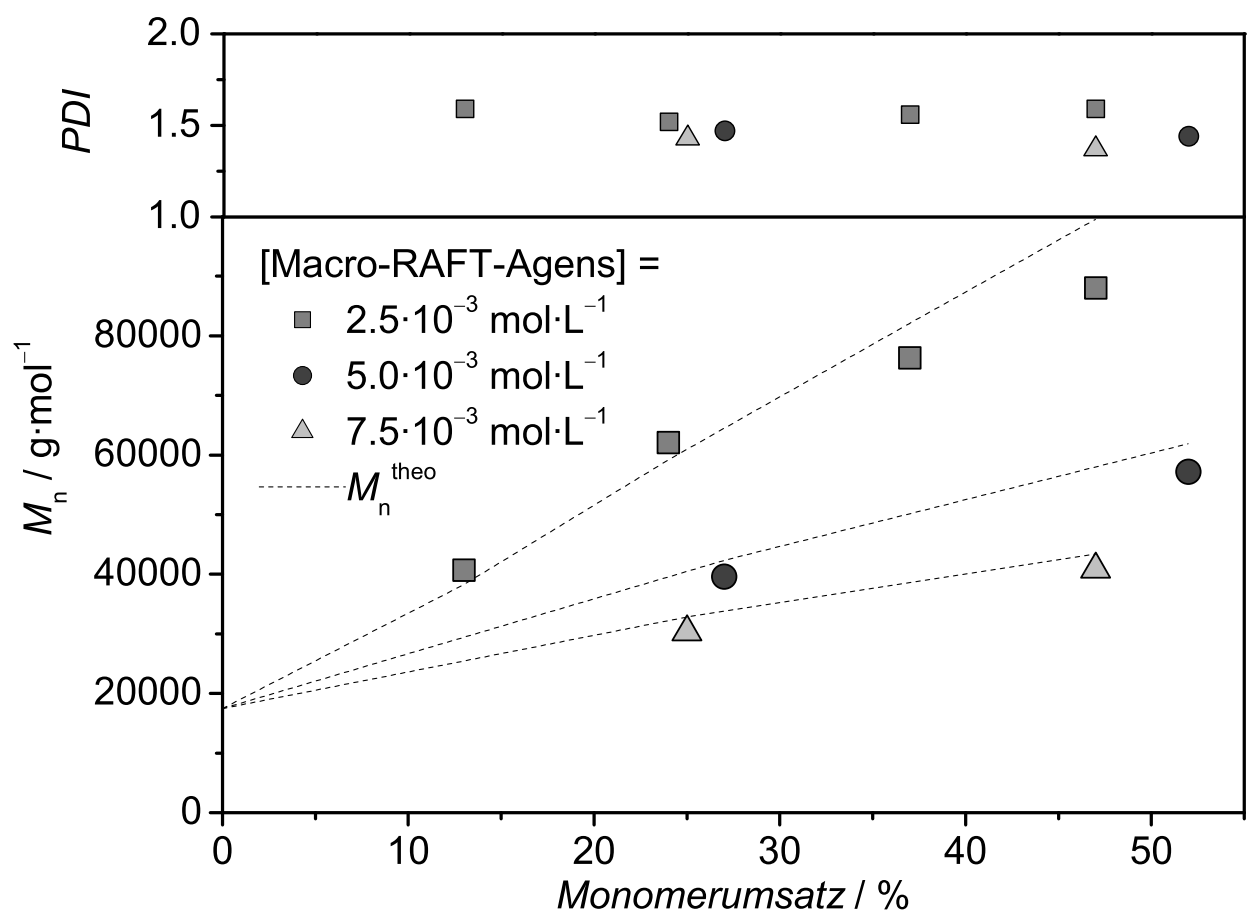

Abbildung 7.11: Auftragung des Zahlenmittels der Molmassen $M_{\mathrm{n}}$ und Polydispersität PDI gegen den Monomerumsatz für die RAFT-Polymerisation von MA bei $60{ }^{\circ} \mathrm{C}$ mit verschiedenen Konzentrationen von Makro-RAFT-Agens (PMMA, $M_{\mathrm{n}}=17.5 \mathrm{~g} / \mathrm{mol}, P D I$ $=1.5$ ) und $0.2 \cdot 10^{-3} \mathrm{~mol} \cdot \mathrm{L}^{-1}$ AIBN. Die gepunkteten Linien stellen die theoretischen Molmassen $M_{\mathrm{n}}^{\text {theo }}$ dar.

Das bifunktionelle RAFT-Agens 8 kann für die einfache Synthese eines Triblockcopolymers beispielsweise PMA-b-PSty-b-PMA eingesetzt werden. Dabei wurde zuerst die RAFT-Polymerisation von Styrol mit 8 durchgeführt (siehe Abbildung 7.13). Im zweiten Schritt wurde die Polymerisation von MA mit dem erhalten MakroRAFT-Agens durchgeführt, um die beiden PMA-Blöcke gleichzeitig hinzuzufügen. Das RAFT-Agens 9 ist genauso geeignet, das Triblockcopolymer PMA-b-PSty-bPMA herzustellen. Dabei wurden die beiden PMA-Blöcke zuerst synthetisiert. Der PSt-Block wurde erst im zweiten Polymerisationsschritt eingebaut. Beide Methoden führten zu den gewünschten Triblockcopolymeren mit kontrollierten Molmassen und niedrigen Polydispersitäten. 


\subsubsection{Unsterbliche RAFT-Polymerisation}

Ein Schwachpunkt der RAFT-Polymerisation, bzw. der kontrollierten radikalischen Polymerisation im Allgemeinen, ist die unvermeidliche Terminierungsreaktion des propagierenden Radikals, was immer zu einer bestimmten Menge an totem Polymer führt. Bei einer idealen RAFT-Polymerisation ist die Radikal-Konzentration genauso groß wie bei einer konventionellen radikalischen Polymerisation. Abhängig vom Stoffmengeverhältnis des primären Radikals zu RAFT-Agens kann die Menge des toten Polymers so beträchtlich sein, dass eine breitere Molmassenverteilung entsteht, und dass eine weitere chemische Modifikation bezüglich Endgruppen oder Blockcopolymerisation nicht mehr sinnvoll ist [155].

Um diesen Schwachpunkt zu umgehen, wurde eine Variation der RAFT-Polymerisation untersucht, die kein totes Polymer in der polymeren Mischung erzeugt. Wir nennen diese Methode „unsterbliche RAFT-Polymerisation“, um die zusätzliche vorteilhafte Eigenschaft dieser Variation hervorzuheben. Dazu wird das bifunktionelle Trithiocarbonat 8 bzw. 10 als RAFT-Agens eingesetzt, bei welchem die gemeinsame Abgangsgruppe sich in der Mitte des RAFT-Agens-Moleküls befindet.
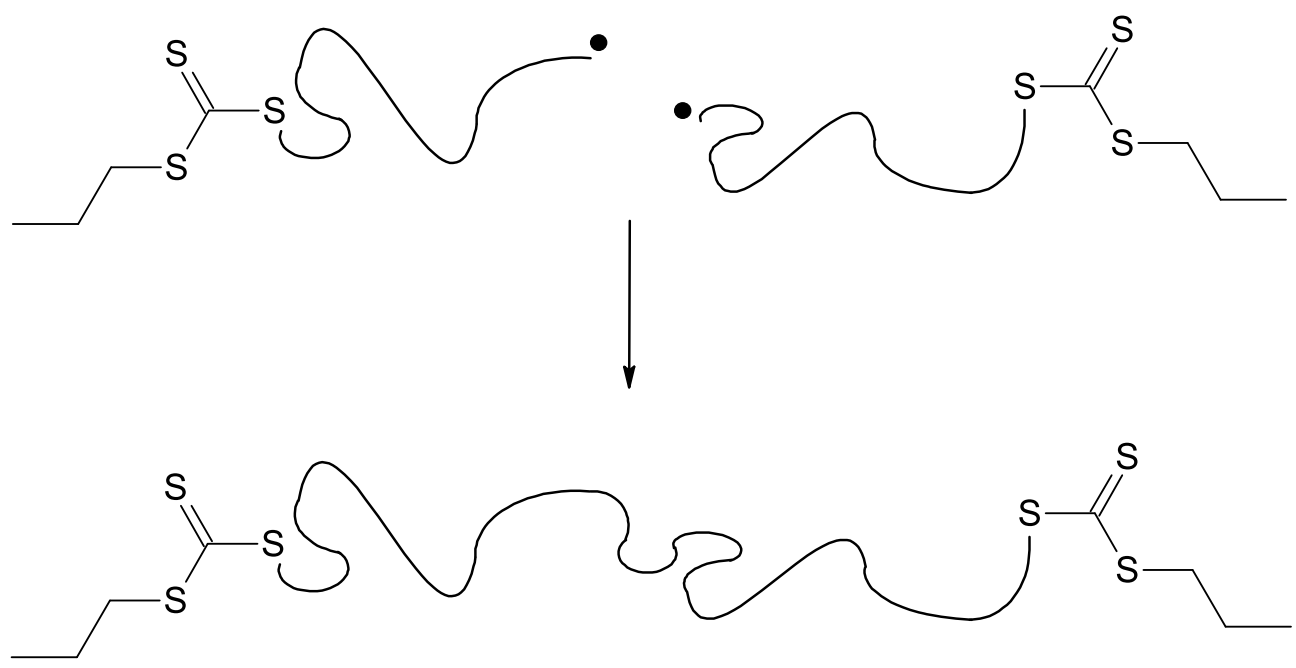

Abbildung 7.12: Grundprinzip der unsterblichen RAFT-Polymerisation. Aus der irreversiblen Terminierungsreaktion zweier propagierender Radikale geht wieder ein lebendes Makro-RAFT-Agens hervor. 
Das Grundprinzip der unsterblichen RAFT-Polymerisation mit 8 ist in Abbildung 7.12 dargestellt. Dabei wird die Terminierungsreaktion zwar nicht unterdrückt, aber durch die Verwendung des bifunktionellen RAFT-Agens 8 ist das Terminierungsprodukt selbst ein Makro-RAFT-Agens und keine tote Polymerkette.

Die positive Auswirkung der unsterblichen RAFT-Polymerisation auf das polymere Produkt kann man anhand von PREDICI ${ }^{\circledR}$-Simulationen verdeutlichen. In Tabelle 7.1 sind die Konzentrationen der beteiligten Spezies und der polymeren Produkte in der Polymerisation mit mono- und bifunktionellem RAFT-Agens zum Vergleich aufgelistet. Dabei steht TT für die lebende Trithiocarbonat-Endgruppe, $I$ für die inaktive Endgruppe und $P$ für die Polymerkette. Man erkennt, dass die Konzentration des toten inaktiven Polymers $[I-P-I]$ bei der Verwendung des bifunktionellen RAFT-Agens verschwindend klein ist. Der Grund dafür ist, dass die Konzentration des Radikals $\left[I-P^{\bullet}\right]$, dessen Terminierung zu totem Polymer führt, um vier Größenordnungen kleiner ist als bei einer normalen RAFT-Polymerisation mit einem monofunktionellen RAFT-Agens.

\begin{tabular}{l|ll}
\hline & Monofunktionell & Bifunktionell \\
\hline$[T T-I]_{0} \mathbf{1}$ & 10.0 & - \\
{$[T T-I-T T]_{0} \mathbf{8}$} & - & 10.0 \\
{$[T T-P-I]$} & 10.0 & 2.86 \\
{$[T T-P-T T]$} & - & 8.57 \\
{$[I-P-I]$} & 1.78 & $2.29 \cdot 10^{-8}$ \\
\hline$\left[T T-P^{\bullet}\right]$ & - & $3.0 \cdot 10^{-5}$ \\
{$\left[I-P^{\bullet}\right]$} & $3.0 \cdot 10^{-5}$ & $2.5 \cdot 10^{-9}$ \\
\hline
\end{tabular}

Tabelle 7.1: Vergleich der Konzentrationen der beteiligten Spezies und der polymeren Produkte in der Polymerisation von Styrol bei Verwendung des monofunktionellen bzw. bifunktionellen RAFT-Agens. Polymerisationsbedingungen für die PREDICI ${ }^{\circledR}$-Simulation sind: Reaktionstemperatur $=$ $60{ }^{\circ} \mathrm{C}$, Reaktionszeit $=10$ Stunden, $[\mathrm{AIBN}]_{0}=5.0 \mathrm{mmol} / \mathrm{L}$.

Abbildung 7.13 zeigt die experimentellen Ergebnisse der unsterblichen RAFTPolymerisation von Styrol mit dem bifunktionellen RAFT-Agens 8. Die mittlere Molmasse steigt, wie erwartet, linear mit dem Monomerumsatz und stimmt sehr gut mit der theoretischen Molmasse überein. Die Polydispersität ist mit Werten um 


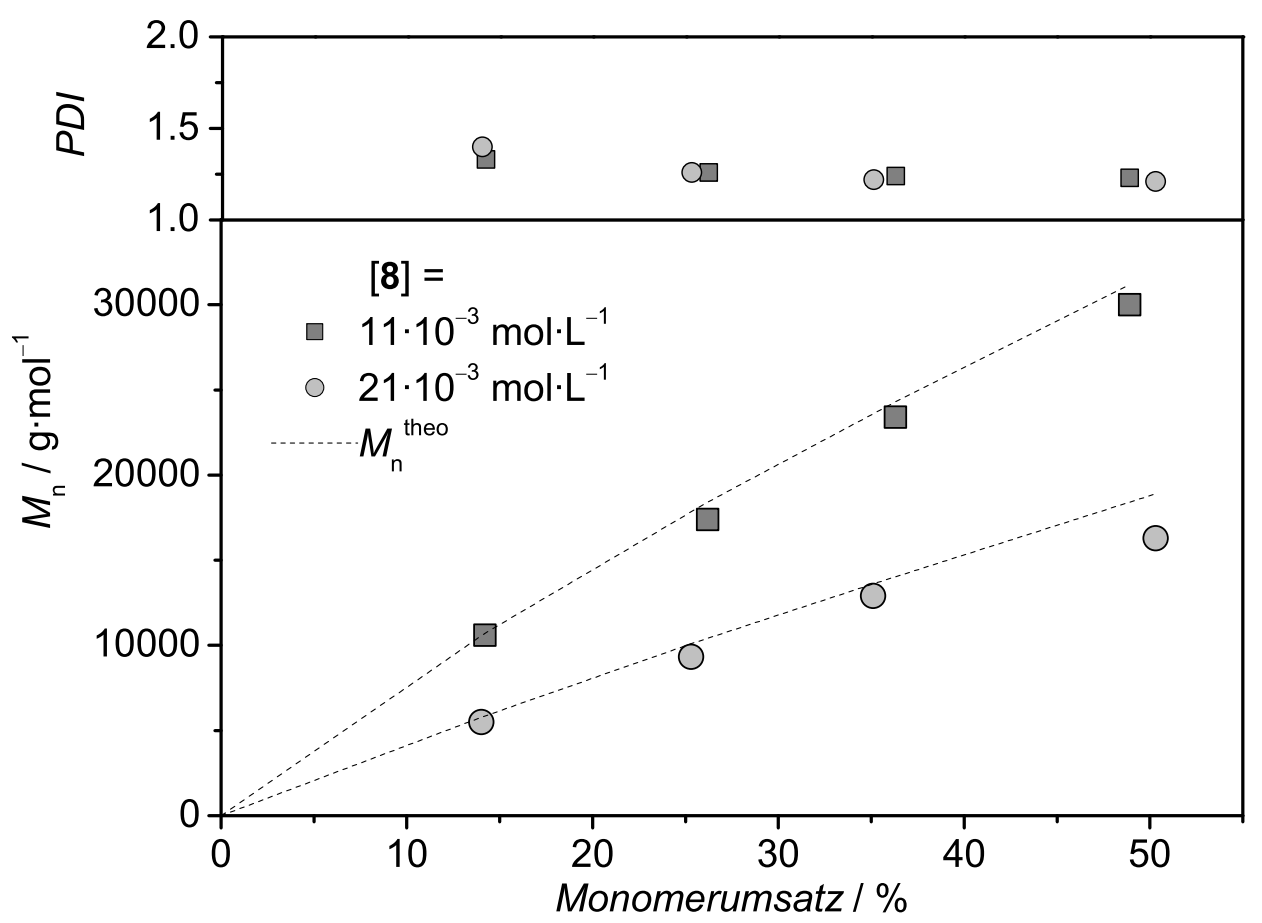

Abbildung 7.13: Auftragung des Zahlenmittels der Molmassen $M_{\mathrm{n}}$ und Polydispersität PDI gegen den Monomerumsatz für die RAFT-Polymerisation von Styrol bei $60{ }^{\circ} \mathrm{C}$ mit verschiedenen Konzentrationen von 8 und $4.5 \cdot 10^{-3} \mathrm{~mol} \cdot \mathrm{L}^{-1}$ AIBN. Die gepunkteten Linien stellen die theoretischen Molmassen $M_{\mathrm{n}}^{\text {theo }}$ dar.

1.2 niedrig. Die Polydispersität des Polymers, das aus der Polymerisation mit dem monofunktionellen RAFT-Agens entsteht, ist mit den Werten 1.4 signifikant größer (vergleiche mit Abbildung 7.5). Durch die unsterbliche RAFT-Polymerisation hat das polymere Produkt eine engere Molmassenverteilung, weil es kein totes Polymermaterial enthält.

\subsubsection{Stern- und Kammpolymere}

In diesem Abschnitt wird ein einfacher Weg zur Herstellung von Stern- und Kammpolymere durch Kondensation der Polymerketten mit einer aktiven Endgruppe vorgestellt. 


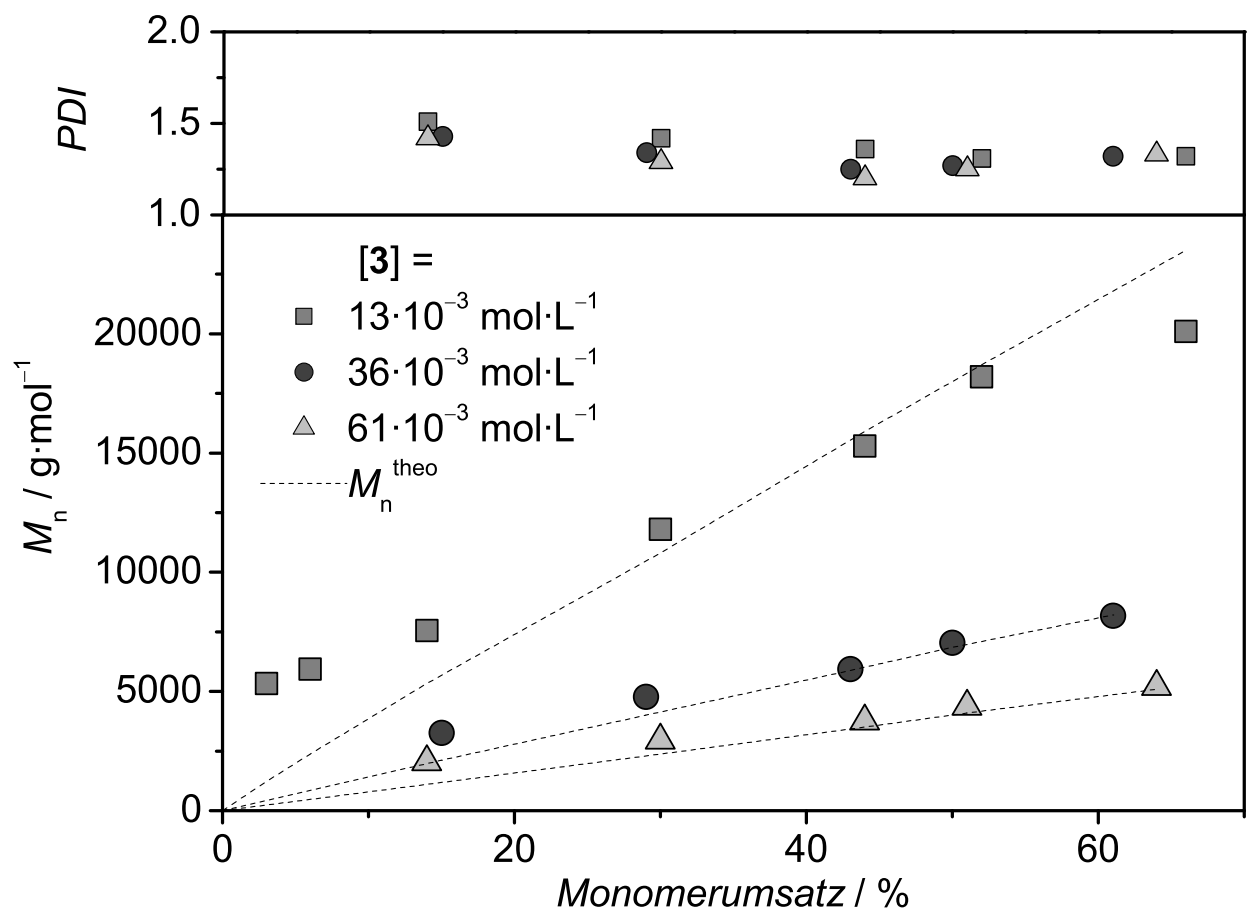

Abbildung 7.14: Auftragung des Zahlenmittels der Molmassen $M_{\mathrm{n}}$ und Polydispersität PDI gegen den Monomerumsatz für die RAFT-Polymerisation von Styrol bei $60{ }^{\circ} \mathrm{C}$ mit verschiedenen Konzentrationen von 3 und 1.9.10 $0^{-3} \mathrm{~mol} \cdot \mathrm{L}^{-1}$ AIBN. Die gepunkteten Linien stellen die theoretischen Molmassen $M_{\mathrm{n}}^{\text {theo }}$ dar.

\section{Synthese des Polymers mit einer aktiven Endgruppe}

Die kontrollierte Polymerisation von Styrol mit dem RAFT-Agens 3 ist in der Abbildung 7.14 dargestellt. Sie zeigt ein Hybridverhalten in der Anfangsphase und ein lineares Wachstum der mittleren Molmasse, die gut mit der theoretischen Molmasse übereinstimmt. Die Polydispersität ist beim Einsetzen einer hohen RAFT-AgensKonzentration sowie einer niedrigen AIBN-Konzentration relativ klein.

Abbildung 7.15 zeigt die kontrollierte Polymerisation von Methylacrylat mit dem RAFT-Agens 3. Die mittlere Molmasse steigt mit dem Monomerumsatz an. Beim Vergleich mit der theoretischen Molmasse sind die experimentellen Werte signifikant größer, was auf eine Kondensation der Polymerkette hinweist. Die Polydispersität ist etwas größer als die Werte aus der Polymerisationsreihe mit dem RAFT-Agens 


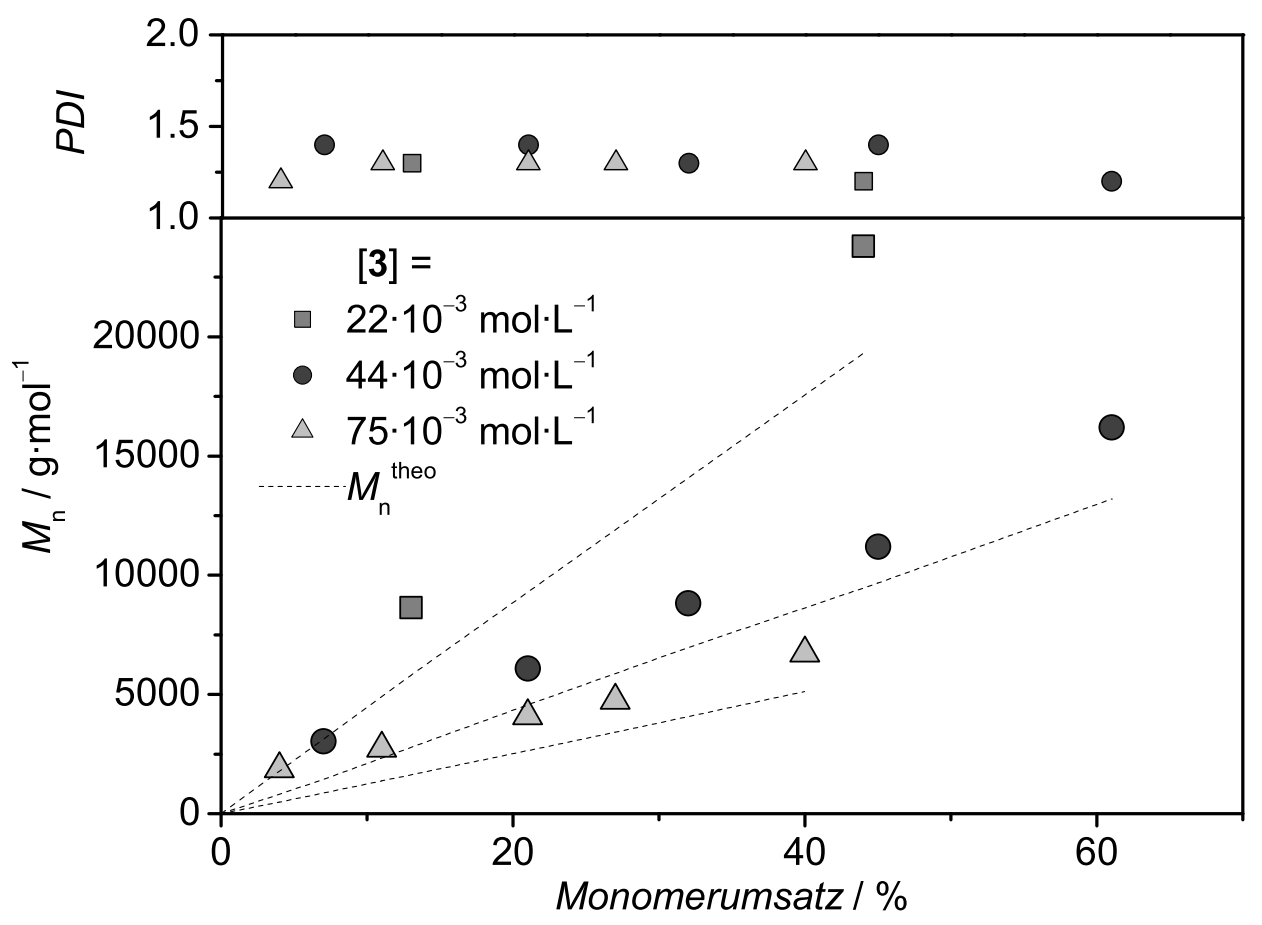

Abbildung 7.15: Auftragung des Zahlenmittels der Molmassen $M_{\mathrm{n}}$ und Polydispersität PDI gegen den Monomerumsatz für die RAFT-Polymerisation von Methylacrylat bei $60{ }^{\circ} \mathrm{C}$ mit verschiedenen Konzentrationen von 3 und $3.4 \cdot 10^{-3} \mathrm{~mol} \cdot \mathrm{L}^{-1}$ AIBN. Die gepunkteten Linien stellen die theoretischen Molmassen $M_{\mathrm{n}}^{\text {theo }}$ dar.

ohne die Trimethoxysilangruppe (vergleiche Abbildung 7.6). Auch dies scheint eine Folge der unkontrollierten Kondensation der reaktiven Polymerketten zu sein.

„Elektrospray-Ionisation“-Massenspektrometrie (ESI-MS) erlaubt eine detaillierte Endgruppenanalyse des Polymers [129, 130]. Diese Massenspektrometrietechnik ist besonders schonend im Hinblick auf den Inonisierungsprozess, sodass die relativ schwach gebundenen Endgruppen wie z. B. Thiocarbonylthio-Gruppen während der Analyse erhalten bleiben [120].

Die Abbildung 7.16 zeigt einen Ausschnitt des Massenspektrums von Polymethylacrylat, das bei der Polymerisation von Methylacrylat mit 3 gebildet wird. Eine Serie von sich wiederholenden Signalen, die einen Abstand von 86.0 Masseneinheiten, d. h. von der Molmasse eines MA-Monomers, haben, ist deutlich sichtbar 


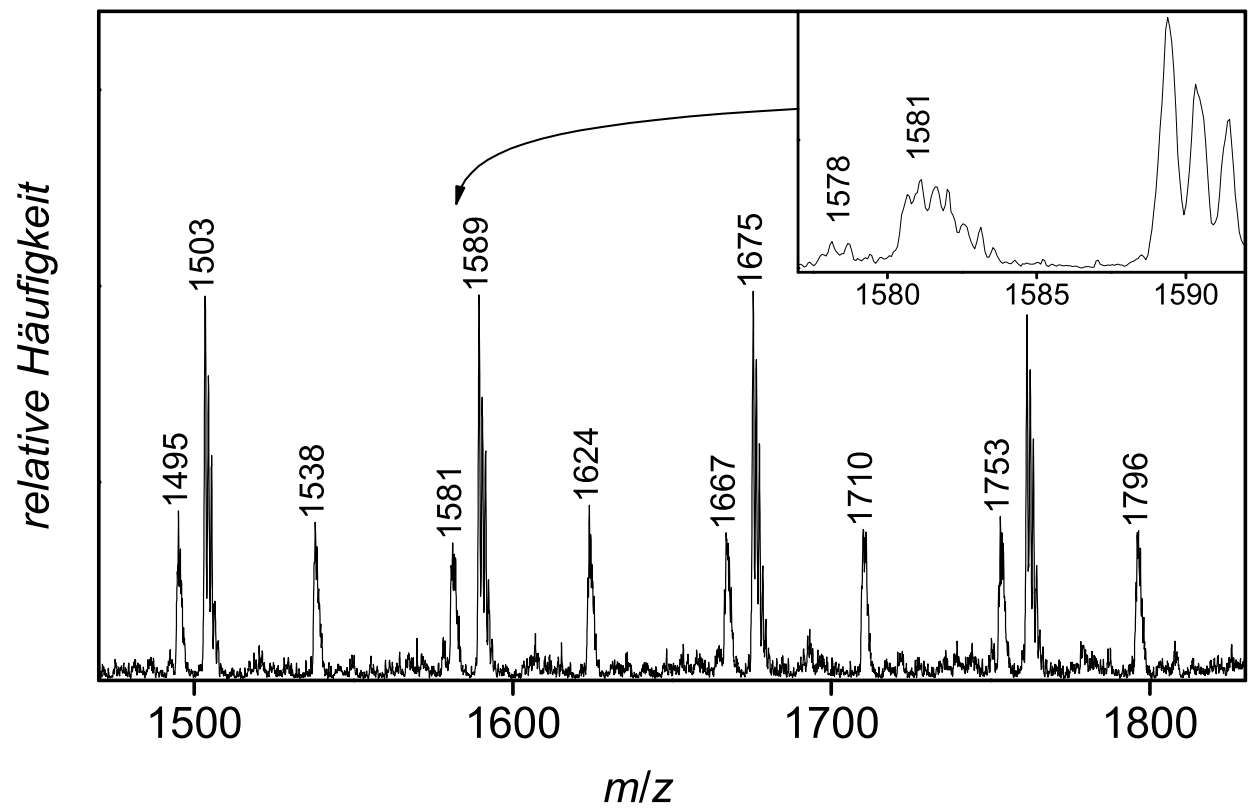

Abbildung 7.16: ESI-MS-Spektrum von $\mathrm{Na}^{+}$-ionisiertem Polymethylacrylat, das aus der Polymerisation von Methylacrylat mit $3\left(44 \cdot 10^{-3} \mathrm{~mol} \cdot \mathrm{L}^{-1}\right)$ und AIBN $\left(3.4 \cdot 10^{-3} \mathrm{~mol} \cdot \mathrm{L}^{-1}\right)$ bei $60{ }^{\circ} \mathrm{C}$ gebildet wird. Der Monomerumsatz betrug nach 20 Minuten Reaktionszeit $6.6 \%$. Das kleine Diagramm stellt die Vergrößerung der Signale um $m / z 1580$ dar.

$(m / z=1503,1589,1675, \ldots)$. Die $m / z$-Werte dieser signalserie weisen auf das Polymethylacrylat, das das RAFT-Agens $\mathbf{3}$ als Endgruppe trägt.

Auch die zweite signalserie, die eine Abstand von 43.0 Masseneinheiten bzw. die Hälfte der Molmasse eines MA-Monomers haben, stammt von Polymethylacrylat, das das RAFT-Agens 3 als Endgruppe trägt. Das Polymer enthielt bei der Ionisierung zwei Natriumkationen, und wurde dadurch zweifach positiv geladen $(z=2)$. Daher halbiert sich der Signalabstand der polymeren Spezies. Auch die Signalabstände der Polymerisotope halbieren sich und betragen anstatt einer Masseneinheit nur noch 0.5 Masseneinheiten (vergleiche die Signalstruktur im kleinen Diagramm in Abbildung 7.16). Dass die Polymere zwei Natriumkationen enthielt, ist ungewöhnlich, da es sonst nicht beobachtet wird. Vermutlich haben die Trimethoxysilangruppen der Polymeren eine hohe Affinität zu Natriumkationen.

Das Polymethylacrylat hat eindeutig definierte Endgruppen. Die Kondensation 
der Polymerkette ist während der RAFT-Polymerisation ausgeblieben. Das kleine Signal bei $m / z=1578$ gehört zu dem Dimer, das aus der Kondensation zweier Polymere entstanden ist. Wie man sieht, ist die Fläche dieses Signals verschwindend klein. Die Strukturen des Polymers und des Dimers sind in der Abbildung 7.18 dargestellt.

\section{Sternpolymer}

Das Polymer mit einer Trimethoxysilangruppe kann miteinander reagieren und zu einem Polymer höherer Molmasse kondensieren. Zuerst werden die Methoxysilangruppen durch die Hydrolyse mit Wasser in Silanol umgewandelt. Die silanolhaltigen Spezies können unter Abspaltung eines Wassermoleküls kondensieren.

Abbildung 7.17 zeigt den Verlauf der Kondensation des linearen Polymers bis hin zum Sternpolymer. Am Anfang enthielt das vorgefertigte Polymer nur wenig Kondensationsprodukte. Nach einem Tag war das Polymer zum Dimer kondensiert, was an der Verschiebung des Maximums der Molmassenverteilung von 16.3 zu $30.8 \mathrm{~kg} / \mathrm{mol}$ ersichtlich ist.

Nach dem dritten Tag hatte das polymere Material durch weitere Kondensation eine höhere Molmasse. Das Maximum der Molmassenverteilung betrug $71.3 \mathrm{~kg} / \mathrm{mol}$, was auf eine Bildung von vielarmigen Sternpolymeren hinweist. Das gebildete Sternpolymer behielt seine Struktur für längere Zeit; weitere Kondensationsschritte fanden nicht mehr statt. Hohe Polymerisationsgrade werden bei der Polykondensation erst bei sehr hohen Monomerumsätzen erreicht, was für Makromonomere wegen der sterischen Abschirmung schwierig zu erreichen scheint (siehe Abbildung 2.5 in Kapitel 2).

Die Frage nach Anzahl der Arme erweist sich als schwierig. Da Sternpolymere ein geringeres hydrodynamisches Volumen als lineare Polymere haben, ist ein quantitativer Vergleich der Molmassenverteilungen, die durch die gleiche Eichung der GPC-Kurven entstanden sind, nicht möglich. Das scheinbare Maximum des Sternpolymers bei $71.3 \mathrm{~kg} / \mathrm{mol}$ hat in Wirklichkeit einen höheren Wert. Die Anzahl des gebildeten Sternpolymers kann daher zwischen 4 und 6 geschätzt werden.

\section{Vorgeschlagener Mechanismus der Kondensation}

Der vorgeschlagene Mechanismus der Kondensation von Polymeren mit einer Trimethoxysilangruppe wird in Abbildung 7.18 schematisch dargestellt. Zuerst konden- 


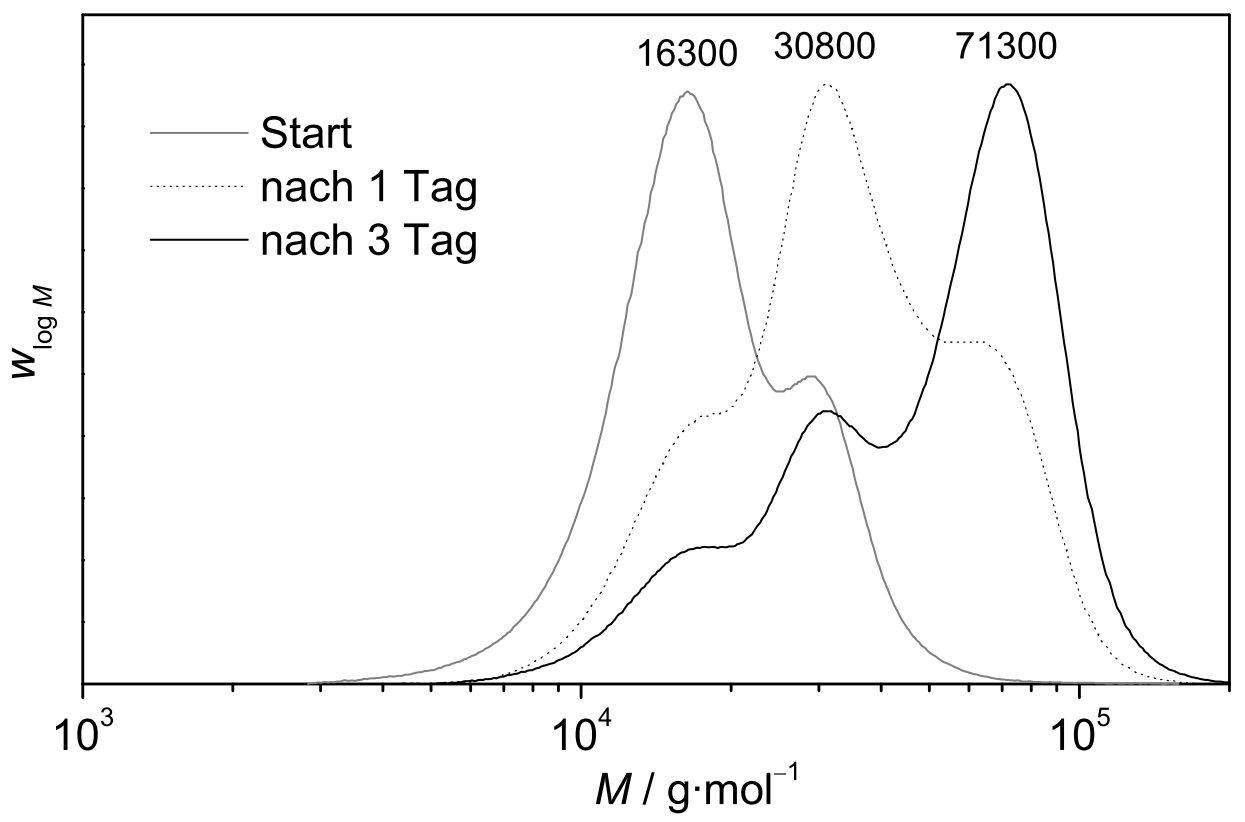

Abbildung 7.17: Molmasseverteilungen gegen der Molmasse auf logarithmischer Skala zur Verfolgung der Sternpolymer-Bildung durch Kondensation. Das Polymer wurde aus einer Reaktion von Methylacrylat mit $3\left(44 \cdot 10^{-3} \mathrm{~mol} \cdot \mathrm{L}^{-1}\right)$ und $\operatorname{AIBN}\left(3.4 \cdot 10^{-3} \mathrm{~mol} \cdot \mathrm{L}^{-1}\right)$ bei $60{ }^{\circ} \mathrm{C}$ gebildet. Vor der Kondensation betrug die mittlere Molmasse des gebildeten Polystyrols $13.0 \mathrm{~kg} / \mathrm{mol}$, während das Maximum seiner engen Molmassenverteilung bei $16.3 \mathrm{~kg} / \mathrm{mol}$ lag. Nach der eintägigen Kondensation lag das Maximum seiner multimodalen Molmassenverteilung bei $30.8 \mathrm{~kg} / \mathrm{mol}$, und nach dem dritten Tag lag es bei $71.3 \mathrm{~kg} / \mathrm{mol}$.

sieren zwei vorgefertigte reaktive Polymere zu einem Dimer, dessen Struktur durch ESI-MS nachgewiesen wurde (siehe Abbildung 7.16).

Zwei Dimere bilden bei einem weiteren Kondensationsschritt ein 4-Arm-Sternpolymer. Die Stabilität des 4-Arm-Sternpolymers kann vor allem durch die sterische Abschirmung der aktiven Silanolgruppen erklärt werden. So können die langen Polymerketten effektiv eine Annäherung der noch vorhandenen Silanolgruppen verhindern, welche sich im Zentrum des polymeren Sterns befinden. Außerdem kann das Zentrum des 4-Arm-Sternpolymer eine stabile Inselsilicat-Struktur bilden, die keine Silanolgruppe mehr enthält (siehe Abbildung 7.18).

Die Kondensation von vorgefertigten reaktiven Polymeren bzw. Dimeren können 


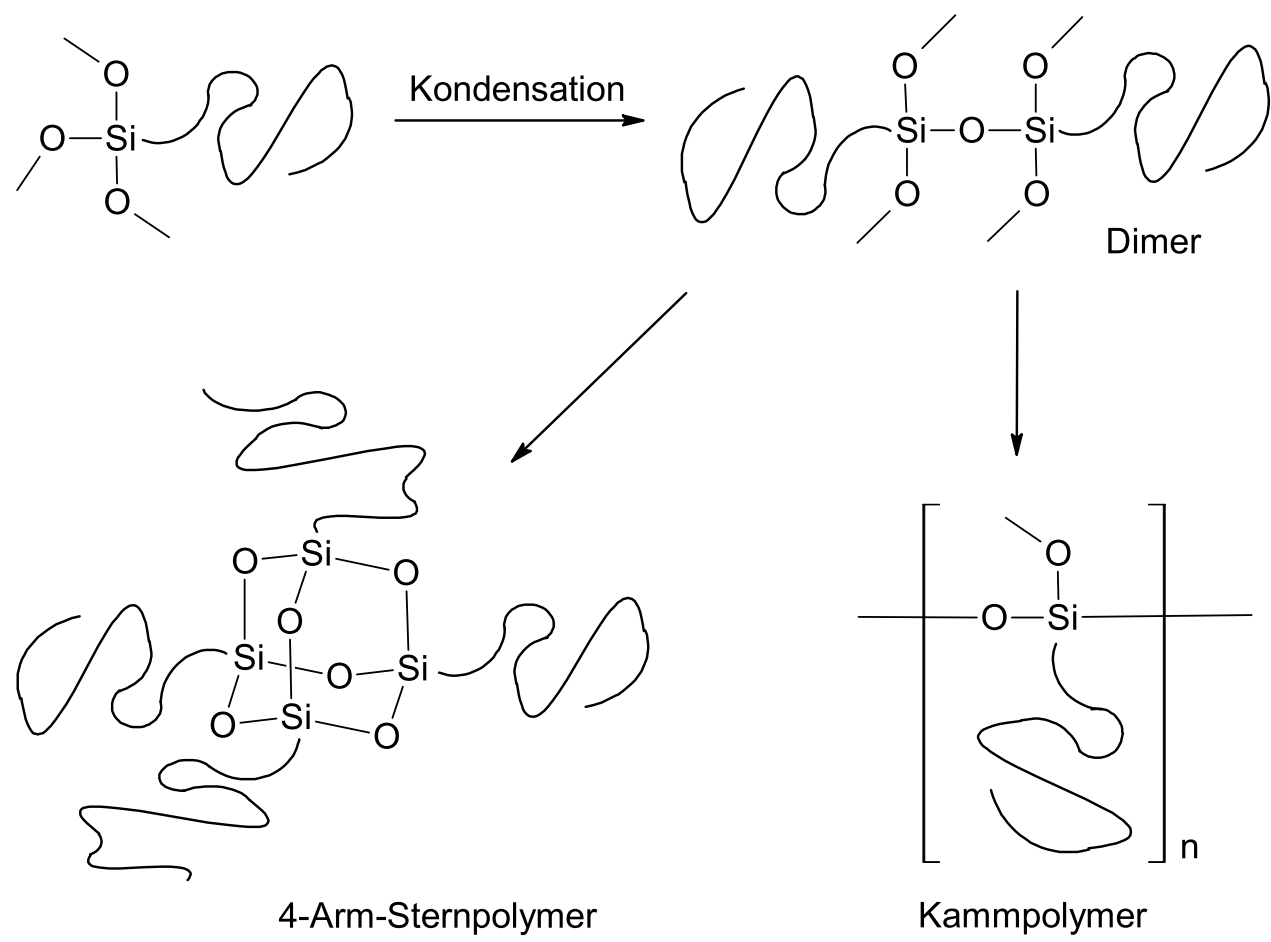

Abbildung 7.18: Vorgeschlagene Mechanismus zur Bildung des Stern- und Kammpolymers aus den einheitlichen Polymerketten mit einer reaktiven Trimethoxysilan-Endgruppe.

zu 5-, 6-Arm-Sternpolymere und Kammpolymere bei höheren Kondensationsgraden führen. Dieser Kondensationsweg hängt sicherlich stark von der Kettenlänge des vorgefertigten Polymers ab. Bei einer kürzeren Kettenlänge ist die Bildung des Kammpolymers wegen der geringen Abschirmung der Polymerketten wesentlich einfacher.

Die vorgestellten Polymere sind aus der Polymerisation mit dem RAFT-Agens 3 synthetisiert. Die gleichen Ergebnisse wurden für das Polymer aus der Polymerisation mit dem RAFT-Agens 5 gefunden ${ }^{3}$.

Die Silangruppe der RAFT-Agenzien 4 und 6 hat nur zwei Methoxygruppen, mit denen man Kammpolymere mit einer linearen Hauptkette synthetisieren kann. Die Aktivität der Endgruppen wird durch die Reduzierung der Kopplungsgruppen

\footnotetext{
${ }^{3}$ Die RAFT-Agenzien 5, 6 und $\mathbf{7}$ können über die Abgangsgruppe $\mathrm{R}$ an einem Substrat wie
} Silica immobilisiert werden. Damit kann man ultradünne Polymerfilme mit einer sehr dichten Oberflächenbeladung herstellen. 


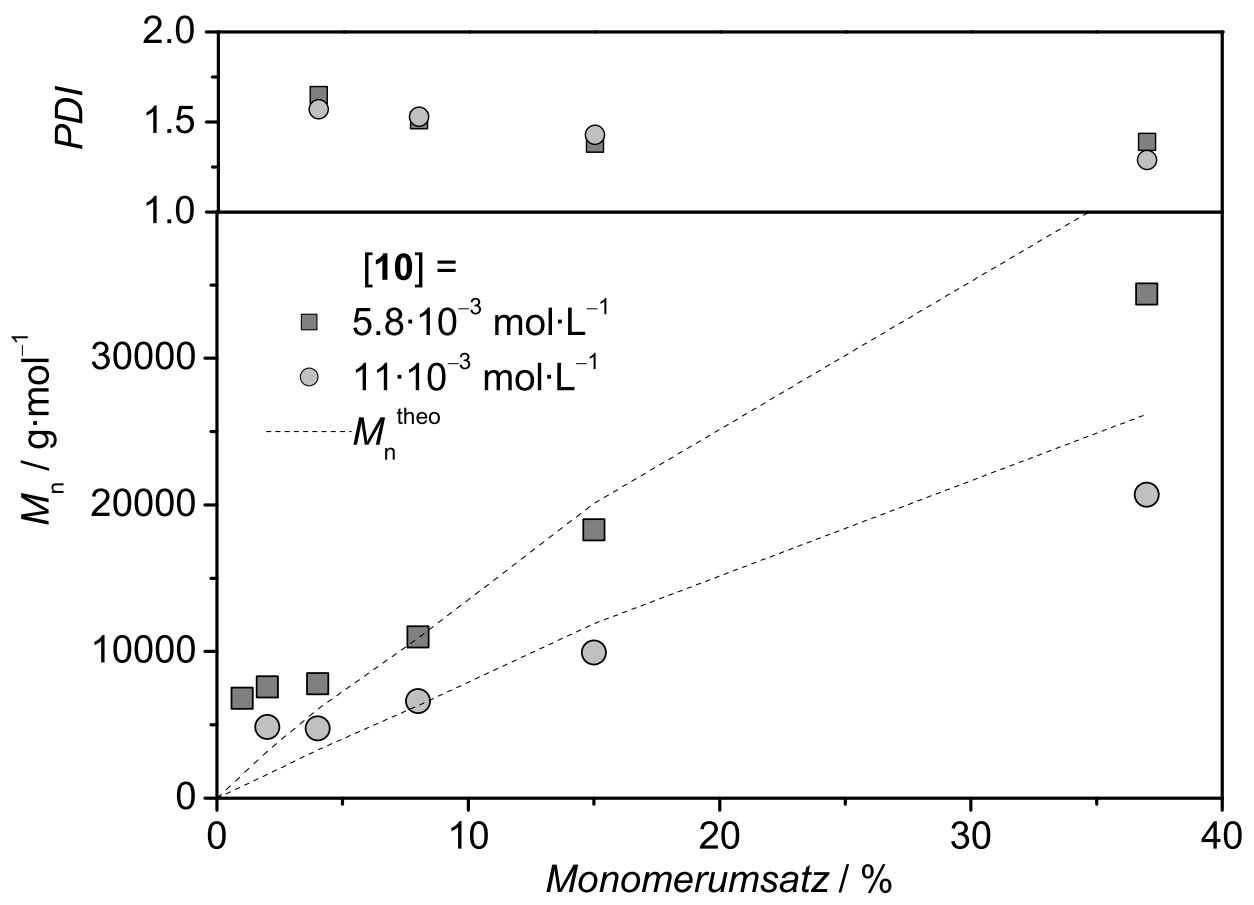

Abbildung 7.19: Auftragung des Zahlenmittels der Molmassen $M_{\mathrm{n}}$ und Polydispersität PDI gegen den Monomerumsatz für die RAFT-Polymerisation von Styrol bei $60{ }^{\circ} \mathrm{C}$ mit verschiedenen Konzentrationen von 10 und 5.0·10 $0^{-3} \mathrm{~mol} \cdot \mathrm{L}^{-1}$ AIBN. Die gepunkteten Linien stellen die theoretischen Molmassen $M_{\mathrm{n}}^{\text {theo }}$ dar.

gesenkt. Weitere Untersuchung und Optimierung der Kondensation der vorgefertigten Polymere wurden nicht weiter verfolgt, da der Schwerpunkt dieser Arbeit auf der radikalischen Polymerisation lag.

\subsubsection{Polymernetzwerk}

Die Ergebnisse der kontrollierten radikalischen Polymerisation von Styrol mit dem bifunktionellen RAFT-Agens 10 sind in der Abbildung 7.19 dargestellt. Die RAFTPolymerisation zeigt für das Styrol das typische Hybridverhalten in der Anfangsphase. Die mittlere Molmasse steigt linear mit dem Monomerumsatz an und stimmt mit der theoretischen Molmasse gut überein. Die Polydispersität ist niedrig, besonders bei hohem Monomerumsatz. 

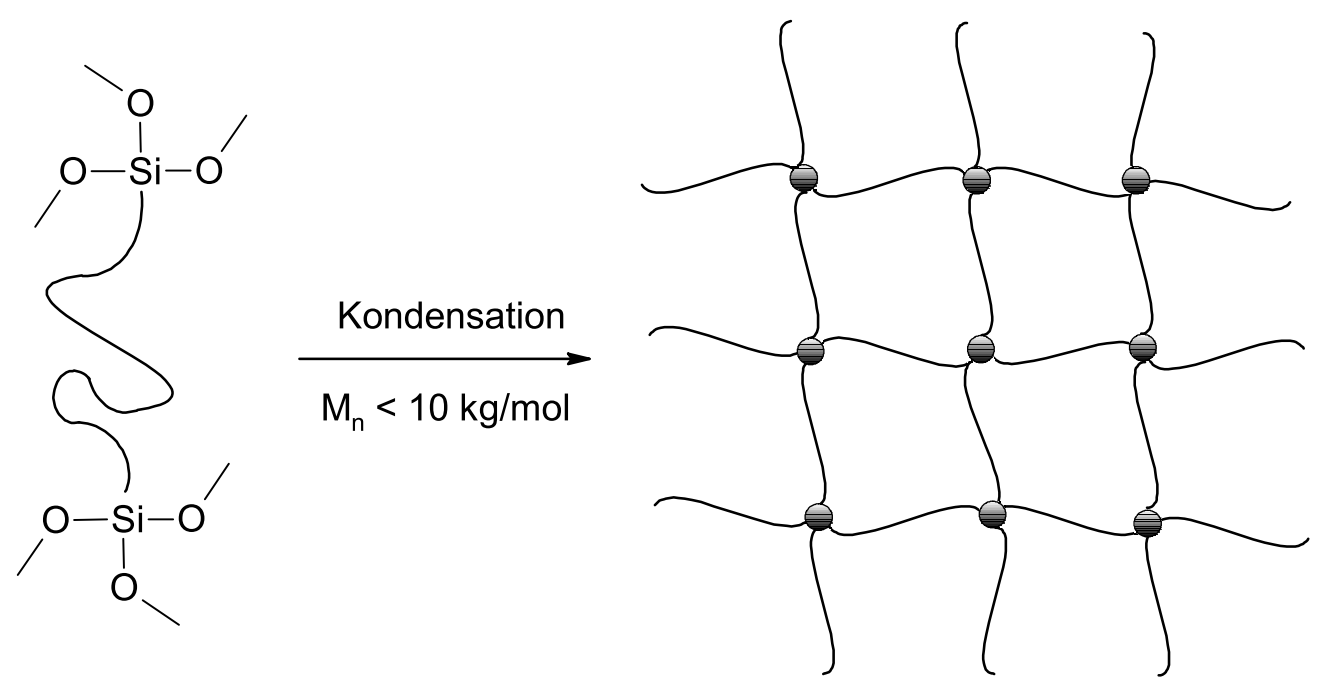

Abbildung 7.20: Bildung des polymeren Netzwerkes aus einheitlichen Polymerketten mit zwei reaktiven Trimethoxysilan-Endgruppen.

Eine ungewöhnliche Eigenschaft des hergestellten Polystyrols ist seine Löslichkeit in Tetrahydrofuran (THF), das normalerweise ein gutes Lösungsmittel für Polystyrol ist. Das Polymer mit einer hohen Molmasse oberhalb von $10 \mathrm{~kg} / \mathrm{mol}$ ist vollständig löslich in THF, während das Polystyrol mit einer mittleren Molmasse unterhalb von $10 \mathrm{~kg} / \mathrm{mol}$ nur unvollständig löslich ist.

Die anormale Unlöslichkeit der kürzeren Polymerketten in THF kann durch die Bildung eines polymeren Netzwerkes erklärt werden (siehe Abbildung 7.20). Wie das RAFT-Agens 10 hat das Polymer zwei reaktive Trimethoxysilangruppe an beiden Kettenenden, die untereinander reagieren und kondensieren können. Die Kondensation der Endgruppen verläuft besonders gut, wenn die Polymerketten kurz sind, denn die lange Polymerkette kann die Kopplungsgruppe effektiv abschirmen und so die Kondensation verhindern. Die kurzen reaktiven Polymerketten bilden ein hochverzweigtes Polymer-Netzwerk, welches nicht mehr löslich ist. Das erhaltene polymere Netzwerk enthält stabile Siloxane als Verknüpfungen, während die einheitlichen Polymerketten gleichmäßige Maschen bilden.

Wie das bifunktionelle RAFT-Agens 10 hat das RAFT-Agens 11 zwei reaktive Trimethoxysilangruppen. Das Makro-RAFT-Agens, das aus der Polymerisation mit dem RAFT-Agens 11 entsteht, kann daher entsprechend kondensieren und ein 
polymeres Netzwerk bilden. Beide RAFT-Agenzien 10 und 11 können an der Oberfläche eines Substrates wie Silica zur Herstellung gebundener polymerer Schleifen immobilisiert werden.

\subsubsection{Immobilisierung des Polymers über "grafting-to“"}

Reaktive Polymerketten können chemisch an Festkörperoberflächen gekoppelt werden, indem über die endständigen reaktiven Gruppen der Polymermoleküle eine kovalente chemische Bindung mit dem Festkörper ausgebildet wird. Die vorgefertigten Polymere mit einer oder zwei Methoxysilangruppen können an der Oberfläche eines Substrates wie Silica chemisch verankert werden. Der Mechanismus der Immobilisierung mit der methoxysilan-haltigen Substanzen wurde bereits in Abbildung 4.3 in Kapitel 4 gezeigt.

Dieser „grafting to“-Weg der Oberflächenmodifikation ist einfach, hat aber den Nachteil, dass die Ausbeuten derartiger Oberflächenreaktionen und somit die erzielbare Oberflächenbeladung der Polymerketten auf der Oberfläche im Allgemeinen nicht sehr hoch sind, denn bereits angebundene Polymermoleküle behindern die Annäherung weiterer Polymerketten an die Oberfläche [156, 157]. Des Weiteren ist das Verfahren auf Polymere mit relativ niedriger Molmasse beschränkt, weil nur bei kürzeren Polymerketten eine genügend hohe Wahrscheinlichkeit dafür besteht, dass sich die Methoxysilangruppe in Reichweite der Anknüpfungsstellen an der Festkörperoberfläche befindet und so eine chemische Reaktion zwischen beiden möglich ist. Für manche Anwendungen wie Stabilisierung eines Nanopartikels ist dieser direkte „arm-first-“-Weg durchaus vorteilhaft.

\subsection{Schlussfolgerung}

Die kontrollierten RAFT-Polymerisationen von Styrol, Methylacrylat bzw. Methylmethacrylat mit den verschiedenen Trithiocarbonat-Verbindungen wurden detailliert untersucht. Die unsterbliche RAFT-Polymerisation mit bifunktionellem RAFTAgens wurde erfolgreich entwickelt, um die Bildung des toten Polymers zu unterdrücken.

Unterschiedliche Strategien zur Herstellung von Copolymeren wurden verfolgt. 
So wurden verschiedene Diblockcopolymere und alternierende Copolymere mit der RAFT-Polymerisationstechnik synthetisiert. Triblockcopolymere ABA wurden mit bifunktionellen RAFT-Agenzien hergestellt. Die Kombination von RAFT-Polymerisation und einmaliger Transferreaktion mit dem thiol-haltigen RAFT-Agens ermöglicht den Einbau von Polymerblöcken, die durch die RAFT-Technik nicht zugänglich sind.

Verschiedene polymere Architekturen wie Stern, Kamm und Netzwerk konnten durch die Kondensation der vorgefertigten reaktiven Polymere synthetisiert werden. Dabei spielten die Kettenlänge der vorgefertigten Polymere und die Anzahl der Kopplungsgruppen beim Verlauf der Kondensation eine entscheidende Rolle.

Mit den vorgestellten Ergebnissen und Diskussionen wurde eine Brücke ausgehend von Homopolymere über Block-, Stern-, Kamm- und Netzwerk-Polymere bis hin zu gebundenen Polymere mit der kontrollierten RAFT-Polymerisationstechnik geschlagen. 


\section{Kapitel 8}

\section{Ausblick}

Das in dieser Arbeit vorgestellte Verfahren der heterogenen RAFT-Polymerisation an festen Oberflächen durch Immobilisierung des RAFT-Agens über die stabilisierende Z-Gruppe sollte weiter optimiert werden. Einflüsse wichtiger Reaktionsparamter wie Lösungsmittel, Oberflächenbeladungsdichte und Konzentrationen der beteiligten aktiven Spezies sollten weiter studiert werden, um an weitere detaillierte Kenntnisse der Dynamik von Polymerketten an festen Oberflächen zu gelangen.

In dieser Arbeit konnten die heterogenen RAFT-Polymerisationen von Styrol, Methylacrylat, Methylmethacrylat und Vinylacetat studiert werden. Die beschriebenen immobilisierten RAFT-Agenzien sollten darüber hinaus auch in den Polymerisation von anderen technisch bedeutsamen Monomeren erprobt werden. Geeignete und viel versprechend erscheinen dafür z. B. Derivate des Styrols, weitere Acrylate und Methacrylate, Acrylamide und Methacrylamide.

Die hergestellten RAFT-Agenzien mit Silan-basierten Verankerungsgruppen können nicht nur an Silica gebunden werden. Die Verwendung anderer Substrate wie Silizium, Metalloxide oder Gold scheinen für weiterführende Anwendungen sehr interessant $\mathrm{zu}$ sein.

Zur Charakterisierung der gebundenen Polymerketten sowie für die Aufklärung der Dynamik der Ketten an der Oberfläche scheint die intensivere Verwendung der Festkörper-NMR-Spektroskopie viel versprechend. Außerdem können physikalische Techniken wie Scanning Elektron Microscopy (SEM), Transmission Elektron Microscopy (TEM) oder die mechanische Spektroskopie einen Beitrag zur Charakterisierung der modifizierten Oberfläche und der Nanopartikel leisten. Es wäre darüber 
hinaus sinnvoll, die einfach durchführbare ATR-FTIR-Spektroskopie für eine schnelle quantitative Analyse der immobilisierten Polymerketten weiterzuentwickeln.

Die hergestellten RAFT-Agenzien mit zwei Trimethoxysilangruppen, die beide an einem Substrat verankert werden können, eignen sich zur Herstellung von Polymerschleifen an der Oberfläche. Gebundene Polymerschleifen konnten bisher nicht hergestellt werden und zeigen womöglich andere Eigenschaften als einfache Polymerketten.

Zur Verwirklichung einer RAFT-Polymerisation, die ausschließlich an der Oberfläche abläuft, erscheint es interessant, nicht nur das RAFT-Agens sondern auch den Initiator an der Oberfläche chemisch zu fixieren. Dazu soll ein geeigneter Initiator mit zwei Verankerungsgruppen synthetisiert werden.

Vom wissenschaftlichen Standpunkt aus betrachtet ist es wichtig, die kinetischen Geschwindigkeitskoeffizienten für die Propagierung und Terminierung der am Substrat gebundenen Radikale zu bestimmen. Das wäre die Voraussetzung für eine richtige Simulation der heterogenen RAFT-Polymerisation.

Es stellt eine Herausforderung dar, geeignete Anwendung der hergestellten polymeren Hybridmaterialen zu finden. Dazu müssen deren Eigenschaften weiter und detaillierter untersucht werden.

Es erscheint außerdem wichtig, die Kondensation der Methoxysilangruppen der vorgefertigten Polymerketten weiter zu studieren. Hinter dieser Methode scheint ein großes Potenzial zur Herstellung neuartiger polymerer Materialien zu stecken. 


\section{Anhang A}

\section{Experimenteller Teil}

In diesem Kapitel werden allgemeine Informationen zur verwendeten Chemikalien und zur Charakterisierung der erhaltenen Produkte dargestellt. Spezielle Synthesetechniken wurden bereits in den einzelnen Kapiteln detailliert beschrieben.

\section{A.1 Chemikalien}

Alle Chemikalien sind - soweit nicht anders angegeben - kommerziell erhältlich (Fluka, Acros, Merck, ABCR) und werden so wie erhaltend und ohne weitere Reinigung verwendet. Die verwendeten Lösungsmittel werden nach den für die Substanzen üblichen Methoden gereinigt und getrocknet.

Bei allen präparativen säulenchromatographischen Trennungen wird Kieselgel 60 (70-230 mesh, 60 A) der Fa. Aldrich eingesetzt. Dünnschichtchromatogramme werden auf Fertigplatten vom Typ „Kieselgel $\mathrm{F}_{254}$ “ der Fa. Merck angefertigt.

\section{A.1.1 Substrate}

\section{Pyrogenes Silica}

Das in diese Arbeit verwendete Silica ist kommerziell erhältlich (fumed Silica, Aldrich). Es wird bei hohen Temperaturen $\left(1000{ }^{\circ} \mathrm{C}\right)$ in einer $\mathrm{H}_{2} / \mathrm{O}_{2}$-Knallgasflamme aus Tetrachlorsilan hergestellt. Das pyrogene Silica zeichnet sich durch kleine und einheitlich geformte Primärpartikel aus. Es besitzt eine nahezu porenfreie Oberfläche. Die Primärpartikel (Größe bis $30 \mathrm{~nm}$ ) der gebildeten Silicate liegen im Partikelbil- 
dungsprozess anfangs separiert vor. Im Verlauf des Partikelwachstums treffen jedoch Primärpartikel aufeinander und werden beim weiteren Wachstum durch chemische Bindung fest miteinander verbunden. Die daraus resultierenden kleinsten frei vorliegenden Partikel werden Aggregat genannt. Die Konzentration und Größe (bis zu $100 \mathrm{~nm}$ ) nehmen mit der Zeit zu, bis durch Zusammenstöße der Aggregate schließlich Agglomerate (Cluster) gebildet werden. Die relativ schwache Wechselwirkung der Aggregate untereinander ermöglicht ein mechanisches Zerstören der Agglomerate. Der Durchmesser der Aggregate bzw. Agglomerate ergibt sich aus der Größe und Anzahl der enthaltenen Primärpartikel. Die Agglomeration ermöglicht eine Trennung der Silica-Nanopartikel vom Lösungsmittel durch einfaches Filtern.

Die spezifische Oberfläche des Silica, gemessen nach der BET-Methode, beträgt nach den Angaben des Herstellers $390 \pm 30 \mathrm{~m}^{2} \cdot \mathrm{g}^{-1}$ für die Primärpartikelgröße von $7 \mathrm{~nm}$. Das Silica weist eine Gesamtsilanolgruppendichte von ca. 2.5 pro $\mathrm{nm}^{2}$ auf.

\section{Polymerer Träger}

Da sich Polystyrol in vielen organischen Solvenzien löst, dienen meist PolystyrolHarze als feste polymere Phase, aber auch Polyethylen und Hexamethylendiaminpolyacryl kommen zum Einsatz. Der polymere Träger besteht aus vernetzten Polymeren, bei denen die Zahl der Vernetzungsstellen so hoch ist, dass alle Polymerketten untereinander zu einem Riesen-Molekül verbunden sind. Kennzeichnend für polymere Netzwerke sind Unlöslichkeit in allen Lösungsmittel, aber reversible Quellbarkeit, deren Maß u. a. abhängig ist von der Vernetzungsdichte, d. h. der Anzahl der Vernetzungsstellen, dem Abstand zwischen den Vernetzungsstellen und der Länge der Vernetzungsbrücken.

Der polymere Träger enthält sogenannte Linker, welche die Anknüpfung von organischen Verbindungen ermöglichen. Neben dem chlormethylierten MerrifielfdHarz kommt besonders das Wang-Harz mit einer terminalen Hydroxy-Gruppe zum Einsatz. Die Konzentration der Anknüpfungsstellen und damit die Beladungsdichte gebundener Verbindungen variiert zwischen 0.1 und $1.2 \mathrm{mmol}$ pro Gramm Harz.

Das in dieser Arbeit verwendete Wang-Harz (Fluka), das ein mit 1 \% Divinylbenzol vernetztes Polystyrol ist, hat eine Partikelgröße von 100 - 200 mesh und eine Konzentration der gebundenen OH-Gruppen von $1.1 \mathrm{mmol} / \mathrm{g}$ Wang-Harz. 


\section{A.1.2 RAFT-Agens}

Die Synthese der RAFT-Agenzien und seine Immobilisierung an einem festen Substrat wurden bereits in den jeweiligen Kapiteln detailliert beschrieben.

\section{A.1.3 Monomere}

Styrol, Methylacrylat, Methylmethacrylat und Vinylacetat werden direkt vor der Polymerisation durch Säulenchromatographie über basisches Aluminiumoxid (Fa. Fluka, aktiviert, Typ 5016 A basisch) vom Inhibitor und Stabilisator gereinigt.

\section{A.1.4 Initiator}

AIBN wird vor der Verwendung aus Methanol umkristallisiert und anschließend im Hochvakuum getrocknet. Man erhält nadelförmige Kristalle. AIBN hat eine Halbwertszeit von fast 18 Stunden bei einer Temperatur von $60{ }^{\circ} \mathrm{C}$. Der langsame Zerfall von AIBN ermöglicht eine gleichbleibende Lieferung des primären Radikals während der Polymerisation.

Alle Initiatoren wurden im Zuge einer Kooperation mit der Firma AKZO NOBEL zur Verfügung gestellt.

\section{A.2 Charakterisierung und Analytik}

\section{A.2.1 GPC-Analyse}

Die Gel-Permations- $C$ hromatographie (GPC) oder Größenausschluß-Chromatographie ( $S$ ize Exclusion $C$ hromatography, SEC) ist die am häufigsten angewandte Methode zur Bestimmung von Molekulargewichten und Molmassenverteilungen von Polymeren. Hierbei erfolgt die Trennung von Polymeren in THF-Lösung nach dem Ausschlussprinzip.

Als Detektionssysteme werden ein Differentialrefraktometer (RI) zur Bestimmung des Brechungsindex und ein UV-Spektrometer eingesetzt. Letzterer setzt eine UV-aktive Gruppe in dem Molekül voraus. Bei dem RI-Detektor ist die Intensität des Signals proportional zur Massen-Konzentration des Polymers in der mobilen Phase. 
Bei der UV-Detektion kann die Absorption in zwei verschiedenen Modi erfolgen. Im Falle eines UV-aktiven Monomers wird ein massenproportionales Signal (z. B. für Polystyrol bei $\lambda=255 \mathrm{~nm}$ ) erhalten; liegt dagegen nur eine UV-aktive Endgruppe vor, ist das Absorptionssignal proportional zur Anzahl der Polymerketten.

Bei der Bestimmung der Molmassenverteilung mittels GPC ist eine Eichung erforderlich, weil es sich um eine Relativmethode handelt. Die Eichung erfolgt mittels engverteilter Polymerstandards, deren mittleren Molmassen durch die Absolutmethoden (Lichtstreuung, Osmometrie) bestimmt wurden.

Wenn ein Polymerstandard nicht zur Verfügung steht, kann die Eichung mittels universeller Kalibrierung erstellt werden. Die Eichkurven zweier Homopolymere können bei Kenntnis der Mark-Houwink-Koeffizienten, die für eine große Anzahl von verschiedenen Monomeren tabelliert sind, ineinander überführt werden. Eine Auswahl für die in dieser Arbeit hergestellten Polymere sind in Tabelle A.1 aufgelistet.

\begin{tabular}{llll}
\hline Polymer & $K / \mathrm{mL} \cdot \mathrm{g}^{-1}$ & $a$ & Ref. \\
\hline Polystyrol & $1.41 \cdot 10^{-2}$ & 0.700 & {$[158]$} \\
Polymethylacrylat & $1.68 \cdot 10^{-2}$ & 0.696 & {$[159]$} \\
Polymethylmethacrylat & $1.29 \cdot 10^{-2}$ & 0.688 & {$[160]$} \\
Polyvinylacetat & $2.24 \cdot 10^{-2}$ & 0.674 & {$[161]$} \\
\hline
\end{tabular}

Tabelle A.1: Mark-Houwink-Koeffizienten $K$ und $a$ für die in dieser Arbeit untersuchten Polymere in THF.

Bei der Bestimmung der Molmassenverteilung von Copolymeren dagegen werden meist nur scheinbare Werte ermittelt, denn eine Bestimmung der Molmasse in Abhängigkeit vom Eluationsvolumen ist nicht eindeutig, da das hydrodynamische Volumen nicht unbedingt linear mit der Copolymerzusammensetzung zusammenhängt.

Die GPC-Analysen werden mit einer Anlage, bestehend aus einer Waters 515 HPLC-Pumpe, einem JASCO AS-2055 Autosampler, einer Säulenkolonne und einem Detektorensystem, durchgeführt. Die Trennung der Proben erfolgt durch eine Vorsäule und drei Trennsäulen (PSS SDV, 8 x 50 mm, $5 \mu$; PSS SDV, 8 x 300 mm, $5 \mu, 10^{5}$ A; PSS SDV, 8 x 300 mm, $5 \mu, 100$ A). Die Detektion der Fraktionen erfolgt an einem RI- (Waters 2410) und einem UV/vis-Detektor (Viskotek VE3210). Die Molmassenverteilungen werden bei $35{ }^{\circ} \mathrm{C}$ mit Tetrahydrofuran als Eluationsmittel 
bei einer Flussgeschwindigkeit von $1.0 \mathrm{~mL} / \mathrm{min}$ und einer Polymerkonzentration von $3 \mathrm{mg} / \mathrm{mL}$ gemessen. Als interner Standard wird Toluol verwendet. Zur Kalibrierung werden eng verteilte Polystyrol- bzw. Polymethylmethacrylatstandards $\left(M_{\mathrm{n}}=400\right.$ bis $3 \cdot 10^{6} \mathrm{~g} \cdot \mathrm{mol}^{-1}$ ) der Firma PSS verwendet. Die erhaltenen Daten werden mit dem Programm PSS WINGPC 6.20 weiter verarbeitet.

\section{A.2.2 FTIR-Spektroskopie}

Die IR-Spektren werden mit dem Spektrometer Bruker IFS 88 erstellt. Das Spektrometer ist mit einem siliciumbeschichteten Calciumfluorid-Strahlteiler, einem InSbund einem DTGS-Detektor, sowie einem Globar und einer Wolfram-Halogenlampe als Lichtquellen ausgestattet, wodurch die Spektrenaufnahme in einem Wellenzahl bereich von 1000 bis $12000 \mathrm{~cm}^{-1}$ möglich ist.

Für die Oberflächenanalyse steht eine ATR-Einheit (MVP 2 Star $^{\text {TM }}$ von Harrick) zur Verfügung, womit die Methode der ATR-IR-Spektroskopie (Attenuated Total Reflection $=$ abgeschwächte Totalreflexion) angewandt werden kann. Als Träger für die Substanzen dient ein Diamantkristall.

Die Registrierung, Fourier-Transformation und Bearbeitung der Spektren erfolgen mit Hilfe der Software Opus von Bruker. Für die Interferogramme werden mindestens 300 Scans zum Erhalt eines hohen Signal-Rausch-Verhältnisses coaddiert. Die Auflösung beträgt hierbei eine oder zwei Wellenzahlen, was mit einem Zerofilling von 2 einen Datenpunktabstand von einer halben bzw. einer Wellenzahl ergibt.

\section{A.2.3 Raman-Spektroskopie}

Die Raman-Spektren werden mit einer Anlage, bestehend aus einem Bruker IFS 66/S und einem Ramanmodull FRA 106/S, durchgeführt. Die Ramananregung erfolgt durch einem Nd:YAG Laser, der mit max. 1.5 Watt betrieben werden kann. Die Wellenzahl des Lasers beträgt $9294.0 \mathrm{~cm}^{-1}$ und die Laserleistung wird bei 1.0 Watt für die im Rahmen dieser Arbeit durchgeführten Messungen eingestellt. Ferner werden ein siliciumbeschichteter Calciumdiflourid-Strahlteiler und ein Ge-Detektor verwendet. Wie beim IR-Spektrometer erfolgen die Registrierung, Fourier-Transformation und weitere Bearbeitung der Spektren über einen integrierten Rechner mit Hilfe des 
Softwareprogrammes Bruker Opus NT. Es werden mindestens 300 Scans bei einer Aufnahmegeschwindigkeit von $5.0 \mathrm{kHz}$ coaddiert.

\section{A.2.4 NMR-Spektroskopie}

Die Lösung-NMR-Spektren werden an einem Varian Mercury 200 aufgenommen. Als Lösungsmittel wird $\mathrm{CDCl}_{3}$ bei einer Probeneinwaage von ca. 1 Gew.\%. verwendet. Die Datenaufnahmezeit liegt im Bereich von Minuten für ${ }^{1} \mathrm{H}-\mathrm{NMR}-$ Spektren und von Stunden für ${ }^{13} \mathrm{C}-\mathrm{NMR}-S p e k t r e n$. Eine Signalreferenzierung erfolgt auf das Signal des zur Probe gegebenen Tetramethylsilan bei 0.0 ppm. Die Spezifizierung der Aufspaltung der Signale erfolgt nach s (singulet), d (dublett), t (triplett), q (quartett), m (multiplett) und b (broad).

Die Festkörper-NMR-Spektren werden am Max-Planck-Institut für Biophysikalische Chemie an einem Wide-Bore Bruker Avance $400 \mathrm{MHz}$ mit „triple-resonance"-MAS-Probenkopf für 2.5 mm-Rotoren aufgenommen. Zum Erhalt eines hohen Signal-Rausch-Verhältnisses werden 76800 Scans coaddiert, was eine Messzeit von zwei vollen Tagen bedeutet.

\section{A.2.5 Massenspektrometrie}

Elektrospray Massenspektrometrie (ESI-MS) wird an einem Finnigan LCQ Ionenfallen-Gerät durchgeführt. Alle Spektren werden in einem Bereich von $m / z=150$ bis 2000 mit einer Sprühspannung von $4.5 \mathrm{kV}$ und einer Kapillartemperatur von $200{ }^{\circ} \mathrm{C}$ aufgenommen. Die Polymerproben werden in einer Mischung von Dichlorethan und Methanol in einer Konzentration von etwa $100 \mu \mathrm{g} \cdot \mathrm{mL}^{-1}$ gelöst. Etwa 200 Spektren werden zum Erhalt eines hohen Signal-Rausch-Verhältnisses coaddiert.

\section{A.2.6 Thermische Analyse}

Die DSC (Differential Scanning Calorimetry) gehört wie die TGA (Thermogravimetrie) zu den thermischen Analysemethoden. Hierbei werden physikalische und chemische Eigenschaften einer Substanz als Funktion der Temperatur gemessen.

Die DSC wird mit einem Gerät (DSC 820) des Herstellers Mettler-Toledo mit angeschlossenem Kryomat RUK 90 (Lauda) bestimmt. Die Messzelle wird mit gas- 
förmigem Stickstoff der Reinheit 4.6 (Linde) bei einem Fluss von $50 \mathrm{~mL} / \mathrm{min}$ gespült. Es werden jeweils Probenmengen zwischen 15 - $20 \mathrm{mg}$ in $40 \mu \mathrm{L}$-Aluminiumtiegeln (Mettler-Toledo) vermessen. Als Referenz dient ein leerer Tiegel. Der Tiegeldeckel wird vor dem Verschließen mit einer Nadel durchstochen, um so einen Gasaustausch mit der Messzellenatmosphäre zu ermöglichen. Es wird ein Messprogramm erstellt, welches zunächst eine Heizphase von 20 bis $150{ }^{\circ} \mathrm{C}$ mit einer Heizrate von $10{ }^{\circ} \mathrm{C} / \mathrm{min}$ durchläuft um die thermische Historie der jeweiligen Proben zu eliminieren. Dem Heizschritt folgt eine Erniedrigung der Temperatur von 150 auf $0{ }^{\circ} \mathrm{C}\left(10^{\circ} \mathrm{C} / \mathrm{min}\right)$. Im letzten Schritt des Programms wird eine erneute Aufheizphase von 0 auf $150{ }^{\circ} \mathrm{C}$ mit einer Heizrate von $10{ }^{\circ} \mathrm{C} / \mathrm{min}$ durchlaufen. Die Glastemperatur wird aus der zweiten Aufheizkurve festgelegt.

Die Thermogravimetrie (TGA) wird mit einem Gerät der Firma TA Instrument vom Typ TGA 2050 untersucht. Die Proben werden mit einer Aufheizrate von $10 \mathrm{~K} / \mathrm{min}$, beginnend bei Raumtemperatur bis $500{ }^{\circ} \mathrm{C}$, gemessen. Hierbei wird die Probenkammer permanent mit einem Stickstoff-Strom von $60 \mathrm{~mL} / \mathrm{min}$ umspült. Dies bedeutet einen pyrolytischen Abbau des organischen Materials unter inerter Stickstoffatmosphäre.

\section{A.2.7 Elementaranalyse}

Die Bestimmungen von Kohlenstoff, Wasserstoff und Stickstoff erfolgen am anorganischen Institut der Universität Göttingen mit einem CHN-O-Rapid Analyzer von Heraeus und von METROHM 662 Photometer mit 636 Tiroprocessor.

Der Schwefelgehalt wird entsprechend der Schöniger-Bestimmung durch das Verbrennen der Substanzen in der „Sauerstoff-Flasche“ und durch die anschließende Titration bestimmt.

\section{A.3 Simulation}

Im Rahmen dieser Arbeit wird die Modellierung der Polymerisation mit Hilfe des Programmpakets PREDICI ${ }^{\circledR}$ (Polyreaction Distribution by Countable System Integration, Version 6.4.2.) durchgeführt. Der Vorteil dieses Programms ist, dass nicht nur die Konzentrationsverläufe, sondern auch die Molmassenverteilungen des entste- 
henden Polymers berechnet werden können. Das Programm nutzt einen diskreten hp-Algorithmus, um komplexe Systeme von Differentialgleichungen zu lösen [80, 162]. Diese effiziente Lösungsmethode ermöglicht eine Durchführung der komplexen Modellierung auf Personalcomputern mit einem vergleichbar geringen Zeitaufwand.

Als Eingabedaten benötigt das Programm ein vollständiges kinetisches Reaktionsschema inklusiv der Geschwindigkeitskoeffizienten sowie die Konzentration der eingesetzten niedermolekularen Verbindungen (Monomer, Initiator, RAFT-Agens etc.). Ein integrierter Interpreter erlaubt die mathematische Beschreibung individueller Zusammenhänge von Geschwindigkeitskonstanten, Konzentrationen und Umsätzen der beteiligten Spezies. Die Ergebnisse der Simulation sind unter anderem die Molmassenverteilungen der polymeren Spezies. Mit dem zusätzlichen Parameterbestimmungsmodull können unbekannte Parameter mit Hilfe der experimentellen Daten angepasst und bestimmt werden. 


\section{Verzeichnis der Abkürzungen}

\begin{tabular}{|c|c|}
\hline $\mathrm{ACCN}$ & $N, N^{\prime}$-Azobiscyclohexancarbonitril \\
\hline AIBN & $N, N^{\prime}$-Azobisisobutyronitril \\
\hline ATR & Abgeschwächte Totalreflexion (eng.: Attenuated Total Reflection) \\
\hline ATRP & Atom Transfer Radical Polymerization \\
\hline$C_{\mathrm{tr}}$ & Übertragungskonstant \\
\hline$c_{\mathrm{x}} ;[\mathrm{X}]$ & Konzentration von $\mathrm{X}$ \\
\hline$c_{\mathrm{x}}^{0} ;[\mathrm{X}]_{0}$ & Einwaagekonzentration von $\mathrm{X}$ \\
\hline CDB & Cumyldithiobenzoat \\
\hline$\delta$ & chemische Verschiebung \\
\hline$d$ & Terminierungsmodus \\
\hline $\mathrm{DSC}$ & Differential Scanning Calorimetry \\
\hline DTBA & Dithiobezoesäure (eng.: Dithiobenzoic acid) \\
\hline ESI-MS & Elektrospray-Ionisation-Massenspektrometrie \\
\hline$f$ & Initiatoreffektivität \\
\hline FTIR & Fourier-Transformation-Infarotspektroskopie \\
\hline GPC & Gel-Permeations-Chromatographie \\
\hline$h$ & Stunde \\
\hline $\mathrm{I}^{\bullet}$ & Primäres Radikal \\
\hline $\mathrm{I}_{2}$ & Initiatormolekül \\
\hline$k_{\mathrm{ad}}$ & Geschwindigkeitskoeffizient der Additionsreaktion \\
\hline$k_{\mathrm{d}}$ & Geschwindigkeitskoeffizient des Initiatorszerfalls \\
\hline$k_{\mathrm{fr}}$ & Geschwindigkeitskoeffizient der Fragmentierungsreaktion \\
\hline$k_{\mathrm{p}}$ & Geschwindigkeitskoeffizient der Propagation \\
\hline$k_{\mathrm{t}}$ & Geschwindigkeitskoeffizient der Terminierung \\
\hline$k_{\mathrm{t}, \mathrm{d}}$ & Geschwindigkeitskoeffizient der Diproportionierung \\
\hline$k_{\mathrm{t}, \mathrm{k}}$ & Geschwindigkeitskoeffizient der Kombination \\
\hline$k_{\mathrm{tr}}$ & Geschwindigkeitskoeffizient der Übertragung \\
\hline
\end{tabular}


$\begin{array}{ll}m & \text { Masse } \\ \text { M } & \text { Monomer } \\ \text { MA } & \text { Methylacrylat }\end{array}$

MADIX Macromolecular Design via the Interchange of Xanthates

$M_{\mathrm{n}} \quad$ Zahlenmittelwert der Molmasse (kurz: mittlere Molmasse)

$M_{\mathrm{n}}^{\text {theo }} \quad$ Theoretische Molmasse

$M_{\mathrm{w}} \quad$ Gewichtsmittelwert der Molmasse

MMA Methylmethacrylat

MW Molekulargewicht (eng.: Molecular Weight)

NMR Kernmagnetische Resonanzspektroskopie

NMP Nitroxide Mediated Polymerization

$\bar{P}_{\mathrm{n}} \quad$ Polymerisationsgrad

$\mathrm{P}_{\mathrm{n}}^{\bullet} \quad$ Propagierendes Polymerradikal

$\mathrm{P}_{\mathrm{n}}-\mathrm{X} \quad$ Schlafende Spezies der kontrollierten Polymerisation

PDI Polydispersitätsindex (kurz: Polydispersität)

PS Polystyrol

PMA Polymethylacrylat

PMMA Polymethylmethacrylat

PPT Propyl(phenylmethyl)trithiocarbonat

PVAc Polyvinylacetat

PVC Polyvinylchlorid

$R_{\mathrm{f}} \quad$ Retention Factor

R-Gruppe Abgangsgruppe eines RAFT-Agens

RAFT Reversible Addition Fragmentation Chain Transfer

RI Brechungsindex (eng.: Refractive Index)

Sty $\quad$ Styrol

$t \quad$ Reaktionszeit

T Temperatur

$T_{\mathrm{g}} \quad$ Glastemperatur

$R_{\mathrm{d}} \quad$ Radikalbildungsgeschwindigkeit

TAPA tert-Amylperoxyacetat

TGA Thermogravimetrische Analyse

THF Tetrahydrofuran

$U_{\mathrm{M}} \quad$ Monomer-Umsatz

VAc Vinylacetat

X Transferagens

Z-Gruppe Stabilisierende Gruppe eines RAFT-Agens 


\section{Literaturverzeichnis}

[1] H. G. Elias, Makromoleküle, wiley-VCH, 1999.

[2] J. Emsley, Parfum, Portwein, PVC ..., Wiley-VCH, 1997.

[3] Amtliche Stastistik, Statistisches Bundesamt, 2007.

[4] K. Matyjaszewski und J. H. Xia, Chemical Reviews, 2001, 2921-2990.

[5] K. Ziegler, Angewandte Chemie, 1936, 499.

[6] M. Szwarc, M. Levy und R. Milkovich, Journal of the American Chemical Society, 1956, 2656-2657.

[7] O. W. Webster, Science, 1991, 887-893.

[8] J. Smid, Journal of Polymer Science Part A - Polymer Chemistry, 2002, 21012107.

[9] J. P. Kennedy, Journal of Polymer Science Part A - Polymer Chemistry, 1999, 2285-2293.

[10] V. C. Gibson und E. L. Marshall, Comprehensive Coordination Chemistry, 2004.

[11] R. R. Schrock, Accounts of Chemical Research, 1990, 158-165.

[12] M. Buback, Makromolekulare Chemie-Macromolecular Chemistry and Physics, 1990, 1575-1587.

[13] J. Qui und K. Matyjaszewski, Acta Polymer, 1997, 169. 
[14] K. Matyjaszewski und A. H. E. Müller, Macromoleculare Nomenclature Note No. 12, 2000.

[15] D. H. Solomon, E. Rizzardo und P. Cacioli, New polymerization process and polymers produced thereby, 1985.

[16] M. K. Georges, R. P. N. Veregin, P. M. Kazmaier und G. K. Hamer, Macromolecules, 1993, 2987-2988.

[17] C. J. Hawker, A. W. Bosman und E. Harth, Chemical Reviews, 2001, 36613688.

[18] J. S. Wang und K. Matyjaszewski, Journal of the American Chemical Society, $1995,5614-5615$.

[19] J. Chiefari, Y. K. Chong, F. Ercole, J. Krstina, J. Jeffery, T. P. T. Le, R. T. A. Mayadunne, G. F. Meijs, C. L. Moad, G. Moad, E. Rizzardo und S. H. Thang, Macromolecules, 1998, 5559-5562.

[20] G. Moad, E. Rizzardo und S. H. Thang, Australian Journal of Chemistry, 2005, 379-410.

[21] H. Fischer, Chemical Reviews, 2001, 3581-3610.

[22] H. Fischer, Macromolecules, 1997, 5666-5672.

[23] Y. Q. Shen, S. P. Zhu, F. Q. Zeng und R. H. Pelton, Macromolecules, 2000, $5427-5431$.

[24] G. Kickelbick, H. J. Paik und K. Matyjaszewski, Macromolecules, 1999, 29412947.

[25] W. Jakubowski und K. Matyjaszewski, Macromolecules, 2005, 4139-4146.

[26] W. Jakubowski, K. Min und K. Matyjaszewski, Macromolecules, 2006, 39-45.

[27] E. Rizzardo, J. Chiefari, B. Y. K. Chong, F. Ercole, J. Krstina, J. Jeffery, T. P. T. Le, R. T. A. Mayadunne, G. F. Meijs, C. L. Moad, G. Moad und S. H. Thang, Macromolecular Symposia, 1999, 291-307. 
[28] R. T. A. Mayadunne, E. Rizzardo, J. Chiefari, Y. K. Chong, G. Moad und S. H. Thang, Macromolecules, 1999, 6977-6980.

[29] C. Barner-Kowollik, M. L. Coote, T. P. Davis, L. Radom und P. Vana, Journal of Polymer Science Part A - Polymer Chemistry, 2003, 2828-2832.

[30] Y. Kwak, A. Goto, Y. Tsujii, Y. Murata, K. Komatsu und T. Fukuda, Macromolecules, 2002, 3026-3029.

[31] M. J. Monteiro und H. de Brouwer, Macromolecules, 2001, 349-352.

[32] Y. Kwak, A. Goto und T. Fukuda, Macromolecules, 2004, 1219-1225.

[33] T. Arita, M. Buback und P. Vana, Macromolecules, 2005, 7935-7943.

[34] C. Barner-Kowollik, J. F. Quinn, D. R. Morsley und T. P. Davis, Journal of Polymer Science Part A - Polymer Chemistry, 2001, 1353-1365.

[35] A. Feldermann, M. L. Coote, M. H. Stenzel, T. P. Davis und C. BarnerKowollik, Journal of the American Chemical Society, 2004, 15915-15923.

[36] C. Barner-Kowollik, P. Vana, J. F. Quinn und T. P. Davis, Journal of Polymer Science Part A - Polymer Chemistry, 2002, 1058-1063.

[37] A. A. Toy, P. Vana, T. P. Davis und C. Barner-Kowollik, Macromolecules, 2004, 744-751.

[38] A. R. Wang und S. P. Zhu, Journal of Polymer Science Part A - Polymer Chemistry, 2003, 1553-1566.

[39] A. Feldermann, A. A. Toy, T. P. Davis, M. H. Stenzel und C. Bamer-Kowollik, Polymer, 2005, 8448-8457.

[40] M. Buback und P. Vana, Macromolecular Rapid Communications, 2006, 12991305.

[41] Y. Kwak, A. Goto, K. Komatsu, Y. Sugiura und T. Fukuda, Macromolecules, 2004, 4434-4440. 
[42] M. Buback, O. Janssen, R. Oswald, S. Schmatz und P. Vana, Macromolecular Symposia, 2007, 158-167.

[43] Y. K. Chong, J. Krstina, T. P. T. Le, G. Moad, A. Postma, E. Rizzardo und S. H. Thang, Macromolecules, 2003, 2256-2272.

[44] J. Chiefari, R. T. A. Mayadunne, C. L. Moad, G. Moad, E. Rizzardo, A. Postma, M. A. Skidmore und S. H. Thang, Macromolecules, 2003, 2273-2283.

[45] M. Destarac, D. Taton, S. Z. Zard, T. Saleh und Y. Six, Advances in Controlled/Living Radical Polymerization, 2003, 536-550.

[46] C. Barner-Kowollik, J. F. Quinn, T. L. U. Nguyen, J. P. A. Heuts und T. P. Davis, Macromolecules, 2001, 7849-7857.

[47] M. Husseman, E. E. Malmstrom, M. McNamara, M. Mate, D. Mecerreyes, D. G. Benoit, J. L. Hedrick, P. Mansky, E. Huang, T. P. Russell und C. J. Hawker, Macromolecules, 1999, 1424-1431.

[48] K. J. Bian und M. F. Cunningham, Journal of Polymer Science Part A Polymer Chemistry, 2005, 2145-2154.

[49] C. Bartholome, E. Beyou, E. Bourgeat-Lami, P. Chaumont, F. Lefebvre und N. Zydowicz, Macromolecules, 2005, 1099-1106.

[50] S. C. Hong, J. F. Lutz, Y. Inoue, C. Strissel, O. Nuyken und K. Matyjaszewski, Macromolecules, 2003, 1075-1082.

[51] J. Yang, S. J. Ding, M. Radosz und Y. Q. Shen, Macromolecules, 2004, 17281734 .

[52] J. V. Nguyen und C. W. Jones, Journal of Catalysis, 2005, 276-294.

[53] S. Edmondson, V. L. Osborne und W. T. S. Huck, Chemical Society Reviews, 2004, 14-22.

[54] S. G. Boyes, A. M. Granville, M. Baum, B. Akgun, B. K. Mirous und W. J. Brittain, Surface Science, 2004, 1-12. 
[55] Y. Tsujii, M. Ejaz, K. Sato, A. Goto und T. Fukuda, Macromolecules, 2001, 8872-8878.

[56] G. Q. Zhai, W. H. Yu, E. T. Kang, K. G. Neoh, C. C. Huang und D. J. Liaw, Industrial und Engineering Chemistry Research, 2004, 1673-1680.

[57] F. Garbassi, M. Morra und E. Occhiello, Polymer Surfaces, Wiley-VCH, 1998.

[58] C.-M. Chan, Polymer Surface Modification and Characterization, Hanser Verlag, 1999.

[59] H. U. Krebs, „Pulsed laser deposition of metals“, in Pulsed Laser Deposition of Thin Films: Applications-Led Growth of Functional Materials, Hg. R. Eason, Wiley-Interscience, 2007 S. 363-382.

[60] H. U. Krebs, M. Weisheit, J. Faupel, E. Süske, T. Scharf, C. Fuhse, M. Störmer, K. Sturm, M. Seibt, H. Kijewski, D. Nelke, E. Panchenko und M. Buback, Adv. Solid State Phys., 2003, 505.

[61] E. Suske, T. Scharf, P. Schaaf, E. Panchenko, D. Nelke, M. Buback, H. Kijewski und H. U. Krebs, Applied Physics A - Materials Science and Processing, 2004, 1295-1297.

[62] E. Suske, T. Scharf, H. U. Krebs, E. Panchenko, T. Junkers, M. Egorov, M. Buback und H. Kijewski, Journal of Applied Physics, 2005, 63501-1-4.

[63] E. Suske, T. Scharf, H. U. Krebs, T. Junkers und M. Buback, Journal of Applied Physics, 2006, 014906-1-4.

[64] S. Perrier und P. Takolpuckdee, Journal of Polymer Science Part A - Polymer Chemistry, 2005, 5347-5393.

[65] M. H. Stenzel, C. Barner-Kowollik, T. P. Davis und H. M. Dalton, Macromolecular Bioscience, 2004, 445-453.

[66] P. Vana, T. R. Davis und C. Barner-Kowollik, Macromolecular Rapid Communications, 2002, 952-956.

[67] M. Baum und W. J. Brittain, Macromolecules, 2002, 610-615. 
[68] L. Barner, J. F. Quinn, C. Barner-Kowollik, P. Vana und T. P. Davis, European Polymer Journal, 2003, 449-459.

[69] C. Yoshikawa, A. Goto, Y. Tsujii, T. Fukuda, K. Yamamoto und A. Kishida, Macromolecules, 2005, 4604-4610.

[70] C. Z. Li und B. C. Benicewicz, Macromolecules, 2005, 5929-5936.

[71] S. Perrier, P. Takolpuckdee und C. A. Mars, Macromolecules, 2005, 6770-6774.

[72] Y. L. Zhao und S. Perrier, Macromolecules, 2006, 8603-8608.

[73] D. Boschmann und P. Vana, Polymer Bulletin, 2005, 231-242.

[74] L. Barner, T. P. Davis, M. H. Stenzel und C. Barner-Kowollik, Macromolecular Rapid Communications, 2007, 539-559.

[75] D. Boschmann und P. Vana, Macromolecules, 2007, 2683-2693.

[76] S. Perrier, C. Barner-Kowollik, J. F. Quinn, P. Vana und T. P. Davis, Macromolecules, 2002, 8300-8306.

[77] J. J. Pesek und M. T. Matyska, Interface Science, 1997, 103-117.

[78] J. V. Nguyen und C. W. Jones, Journal of Polymer Science Part A - Polymer Chemistry, 2004, 1384-1399.

[79] M. Hesse, H. Meier und B. Zeeh, Spektroskopische Methode in der organischen Chemie, 2005.

[80] M. Wulkow, Macromolecular Theory and Simulations, 1996, 393-416.

[81] P. Vana, T. P. Davis und C. Barner-Kowollik, Macromolecular Theory and Simulations, 2002, 823-835.

[82] E. F. Vansant, P. Van Der Voort und K. C. Vrancken, Characterization and Chemical Modification of the Silica Surface, 1995.

[83] M. D. Zammit, T. P. Davis, D. M. Haddleton und K. G. Suddaby, Macromolecules, 1997, 1915-1920. 
[84] R. K. Bai, Y. Z. You und C. Y. Pan, Polymer International, 2000, 898-902.

[85] D. H. Nguyen und P. Vana, Australian Journal of Chemistry, 2006, 549-559.

[86] P. Takolpuckdee, C. A. Mars und S. Perrier, Organic Letters, 2005, 3449-3452.

[87] B. Arkles, CHEMTECH, 1977, 766-778.

[88] J. Steinmetz und H. Mottola, Chemically Modified Surfaces, Elsevier, 1992.

[89] J. Blitz und C. Little, Fundamental and Applied Aspects of Chemically Modified Surfaces, Royal Society of Chemistry, 1999.

[90] E. A. Grulke, J. Brandrup und E. H. Immergut, Polymer Handbook, 1999.

[91] M. A. Gallop und W. L. Fitch, Current Opinion in Chemical Biology, 1997, 94-100.

[92] M. Baldus, Angewandte Chemie - International Edition, 2006, 1186-1188.

[93] C. E. Hughes und M. Baldus, Annual Reports on NMR Spectroscopy, Vol 55, 2005, 121-158.

[94] T. G. Fox und P. G. Flory, Journal of Applied Physic, 1950, 581.

[95] B. J. Ash, R. W. Siegel und L. S. Schadler, Journal of Polymer Science Part B - Polymer Physics, 2004, 4371-4383.

[96] A. Bansal, H. C. Yang, C. Z. Li, R. C. Benicewicz, S. K. Kumar und L. S. Schadler, Journal of Polymer Science Part B - Polymer Physics, 2006, 29442950.

[97] E. Dalnoki-Veress, J. A. Forrest, C. Murray, C. Gigault und J. R. Dutcher, Physical Review E, 2001,--.

[98] C. Becker, H. Krug und H. Schmidt, Journal of Sol - Gel Science and Technology, 1997, 625-627.

[99] W. D. Hergeth, U. J. Steinau, H. J. Bittrich, G. Simon und K. Schmutzler, Polymer, 1989, 254-258. 
[100] F. K. Liu, S. Y. Hsieh, F. H. Ko und T. C. Chu, Colloids and Surfaces A Physicochemical and Engineering Aspects, 2003, 31-38.

[101] V. Arrighi, I. J. McEwen, H. Qian und M. B. S. Prieto, Polymer, 2003, 62596266.

[102] J. A. Forrest, K. DalnokiVeress und J. R. Dutcher, Physical Review E, 1997, $5705-5716$.

[103] B. J. Ash, L. S. Schadler und R. W. Siegel, Materials Letters, 2002, 83-87.

[104] A. Bansal, H. C. Yang, C. Z. Li, K. W. Cho, B. C. Benicewicz, S. K. Kumar und L. S. Schadler, Nature Materials, 2005, 693-698.

[105] P. Corpart, D. Charmot, T. Biadatti, S. Z. Zard und D. Michelet, Int. Pat. Appl., 1998, WO 9858974.

[106] D. Charmot, P. Corpart, H. Adam, S. Z. Zard, T. Biadatti und G. Bouhadir, Macromolecular Symposia, 2000, 23-32.

[107] M. C. Iovu und K. Matyjaszewski, Macromolecules, 2003, 9346-9354.

[108] M. Destarac, D. Charmot, X. Franck und S. Z. Zard, Macromolecular Rapid Communications, 2000, 1035-1039.

[109] M. H. Stenzel, L. Cummins, G. E. Roberts, T. R. Davis, P. Vana und C. Barner-Kowollik, Macromolecular Chemistry and Physics, 2003, 1160-1168.

[110] D. H. Nguyen und P. Vana, Polymers for Advanced Technologies, 2006, 625633.

[111] A. Favier und M. T. Charreyre, Macromolecular Rapid Communications, 2006, 653-692.

[112] R. B. Merrifield, Journal of the American Chemical Society, 1963, 2149-2153.

[113] C. Pinilla, J. Appel, S. Blondelle, C. Dooley, B. Dorner, J. Eichler, J. Ostresh und R. A. Houghten, Biopolymers, 1995, 221-240. 
[114] L. Gold, B. Polisky, O. Uhlenbeck und M. Yarus, Annual Review of Biochemistry, 1995, 763-797.

[115] M. R. Wood, D. J. Duncalf, S. P. Rannard und S. Perrier, Organic Letters, 2006, 553-556.

[116] S. Hinrichs, Messung und Modellierung der Initiatoreffektivität organischer Peroxide in der Ethen-Hochdruckpolymerisation, Doktorarbeit, 2005.

[117] T. M. Legge, A. T. Slark und S. Perrier, Journal of Polymer Science Part A - Polymer Chemistry, 2006, 6980-6987.

[118] R. K. Bai, Y. Z. You, P. Zhong und C. Y. Pan, Macromolecular Chemistry and Physics, 2001, 1970-1973.

[119] R. Plummer, Y. K. Goh, A. K. Whittaker und M. J. Monteiro, Macromolecules, $2005,5352-5355$.

[120] P. Vana, L. Albertin, L. Barner, T. P. Davis und C. Barner-Kowollik, Journal of Polymer Science Part A - Polymer Chemistry, 2002, 4032-4037.

[121] Y. K. Goh, M. R. Whittaker und M. J. Monteiro, Journal of Polymer Science Part A - Polymer Chemistry, 2005, 5232-5245.

[122] S. Oae, T. Okabe und T. Yagihara, Tetrahedron, 1972, 3203.

[123] A. Dureault, Y. Gnanou, D. Taton, M. Destarac und F. Leising, Angewandte Chemie - International Edition, 2003, 2869-2872.

[124] J. T. Xu, J. P. He, D. Q. Fan, W. Tang und Y. L. Yang, Macromolecules, 2006, $3753-3759$.

[125] C. Barner-Kowollik, M. Buback, B. Charleux, M. L. Coote, M. Drache, T. Fukuda, A. Goto, B. Klumperman, A. B. Lowe, J. B. Mcleary, G. Moad, M. J. Monteiro, R. D. Sanderson, M. P. Tonge und P. Vana, Journal of Polymer Science Part A - Polymer Chemistry, 2006, 5809-5831.

[126] M. Benaglia, E. Rizzardo, A. Alberti und M. Guerra, Macromolecules, 2005, 3129-3140. 
[127] M. Buback, T. Junkers und P. Vana, Macromolecular Rapid Communications, 2005, 796-802.

[128] X. S. Chai, F. J. Schork und E. M. Oliver, Journal of Applied Polymer Science, 2006, 1471-1475.

[129] J. B. Fenn, Angewandte Chemie - International Edition, 2003, 3871-3894.

[130] M. Buback, H. Frauendorf und P. Vana, Journal of Polymer Science Part A Polymer Chemistry, 2004, 4266-4275.

[131] P. Vana, T. P. Davis und C. Barner-Kowollik, Australian Journal of Chemistry, $2002,315-318$.

[132] X. L. Jiang, P. J. Schoenmakers, J. L. J. van Dongen, X. W. Lou, V. Lima und J. Brokken-Zijp, Analytical Chemistry, 2003, 5517-5524.

[133] P. Vana, C. Barner-Kowollik, T. P. Davis und K. Matyjaszewski, Radical Polymerization, Bd. 11 von Encyclopedia of Polymer Science, 2004.

[134] F. Jahanzad, M. Kazemi, S. Sajjadi und F. A. Taromi, Polymer, 1993, 35423544 .

[135] P. M. Kazmaier, K. A. Moffat, M. K. Georges, R. P. N. Veregin und G. K. Hamer, Macromolecules, 1995, 1841-1846.

[136] G. Levesque, G. Tabak und F. Outurquin, Comptes Rendus Hebdomadaires Des Seances De L Academie Des Sciences Serie C, 1975, 1289-1292.

[137] D. G. Hawthorne, G. Moad, E. Rizzardo und S. H. Thang, Macromolecules, $1999,5457-5459$.

[138] S. H. Thang, Y. K. Chong, R. T. A. Mayadunne, G. Moad und E. Rizzardo, Tetrahedron Letters, 1999, 2435-2438.

[139] M. Drache, G. Schmidt-Naake, M. Buback und P. Vana, Polymer, 2005, 84838493.

[140] W. Vogt und L. Dulog, Makromolekulare Chemie, 1969, 223. 
[141] J. P. Vanhook und A. V. Tobolsky, Journal of Polymer Science, 1958, 429-445.

[142] H. Fischer und L. Radom, Angewandte Chemie - International Edition, 2001, $1340-1371$.

[143] S. Beuermann, M. Buback, T. P. Davis, R. G. Gilbert, R. A. Hutchinson, O. F. Olaj, G. T. Russell, J. Schweer und A. M. vanHerk, Macromolecular Chemistry and Physics, 1997, 1545-1560.

[144] H. Fischer und H. Paul, Accounts of Chemical Research, 1987, 200-206.

[145] G. Johnston-Hall, A. Theis, M. J. Monteiro, T. P. Davis, M. H. Stenzel und C. Barner-Kowollik, Macromolecular Chemistry and Physics, 2005, 2047-2053.

[146] G. Moad, J. Chiefari, Y. K. Chong, J. Krstina, R. T. A. Mayadunne, A. Postma, E. Rizzardo und S. H. Thang, Polymer International, 2000, 993-1001.

[147] A. R. Kannurpatti, S. X. Lu, G. M. Bunker und C. N. Bowman, Macromolecules, 1996, 7310-7315.

[148] T. Otsu, Journal of Polymer Science Part A - Polymer Chemistry, 2000, 21212136.

[149] M. L. Coote, G. P. F. Wood und L. Radom, Journal of Physical Chemistry A, 2002, 12124-12138.

[150] T. Otsu, T. Ito und M. Imoto, Journal of Polymer Science Part A - Polymer Chemistry, 1966, 733 .

[151] P. C. Jocelyn, Biochemistry of the SH Group, Academic Press: London, 1972.

[152] J. Liu, V. Bulmus, C. Barner-Kowollik, M. H. Stenzel und T. P. Davis, Macromolecular Rapid Communications, 2007, 305-314.

[153] N. C. Kalarickal, S. Rimmer, P. Sarker und J. C. Leroux, Macromolecules, 2007, 1874-1880.

[154] B. J. Kim, S. Given-Beck, J. Bang, C. J. HawkerO und E. J. Kramer, Macromolecules, 2007, 1796-1798. 
[155] Y. K. Goh und M. J. Monteiro, Macromolecules, 2006, 4966-4974.

[156] H. Q. Huang, S. E. Rankin, A. Cammers und L. S. Penn, Abstracts of Papers of the American Chemical Society, 2005, U4133-U4134.

[157] H. Q. Huang, A. Cammers und L. S. Penn, Macromolecules, 2006, 7064-7070.

[158] C. Strazielle, H. Benoit und O. Vogl, European Polymer Journal, 1978, 331334.

[159] M. Buback, A. Feldermann, C. Barner-Kowollik und I. Lacik, Macromolecules, 2001, 5439-5448.

[160] M. Stickler, D. Panke und W. Wunderlich, Makromolekulare Chemie - Macromolecular Chemistry and Physics, 1987, 2651-2664.

[161] S. Beuermann, M. Buback und D. Nelke, Macromolecules, 2001, 6637-6640.

[162] R. Tegmann und M. Wulkow, Benutzerhandbuch Predici, 1996. 


\section{Danksagung}

An dieser Stelle möchte ich Prof. Dr. M. Buback für die Überlassung des interessanten Themas und die immerwährende Unterstützung dieser Arbeit meinen tiefen Dank aussprechen.

Ganz besonders bedanken möchte ich mich bei meinem Betreuer, Dr. P. Vana, für die großartige Unterstützung. Während der Durchführung dieser Arbeit ist Dr. P. Vana immer mit detailliertem Wissen, großem Interesse und viel Geduld auf meine zahlreichen Fragen eingegangen.

Bei Prof. Dr. H. U. Krebs bedanke ich mich für seine Unterstützung bei Charakterisierung von Oberflächen und für die Übernahme des Korreferats.

Ferner danke ich Prof. Dr. M. Suhm, stellvertretend für das durch die Deutsche Forschungsgemeinschaft (DFG) geförderte Graduiertenkolleg (GRK 782, „Spektroskopie und Dynamik molekularer Knäuel und Aggregate“), das mich finanziell unterstützt hat.

Besonders bedanken möchte ich bei Herrn Dr. H. P. Vögele, der mir stets bei apparativen Schwierigkeiten und Rechnerfragen geholfen hat.

Mein besonderer Dank gilt auch Dr. S. Perrier und und seiner Arbeitsgruppe für die schöne und erfolgreiche Zeit in Leeds, England.

Für die Festkörper-NMR-Messungen möchte ich Dr. Baldus und R. Schneider vom Max-Planck-Institut für Biophysikalische Chemie danken.

Für die Hilfe bei der physikalischen Oberflächenanalyse und die schöne Projektwoche 
danke ich T. Scharf und A. Meschede vom Institut für Materialphysik.

Bei Herrn Dr. H. Frauendorf möchte ich mich für die freundliche Hilfe bei ESI-MSMessungen bedanken.

D. Boschmann, F. Günzler, O. Janßen, M. Mänz und P. Vana möchte ich für das aufmerksame Korrekturlesen dieser Arbeit herzlich danken.

Mein Dank gilt H. Rohmann für die freundliche Unterstützung bei der Durchführung der Experimente.

Allen Mitgliedern der Arbeitsgruppe Buback danke ich für die freundschaftliche Zusammenarbeit und stete Hilfsbereitschaft. Spezieller Dank gilt meinen aktuellen und ehemaligen Bürokollegen M. Gadermann, F. Günzler, P. Hesse, M. Imran-Ulhag, O. Janßen, S. Kukuckova, B. Springer und T. Theis dafür, dass wir gute und schlechte Zeiten zusammen erlebt haben.

Für die schnelle und zuverlässige Arbeit durch die Glasbläserei und die Werkstätten des Institutes möchte ich stellvertretend Herrn V. Meyer und Herrn H.-J. Schlette danken.

Große Dank gilt der NMR-Abteilung und der Chemikalienausgabe des Institutes für Organische und Biomolekulare Chemie sowie der Analytischen Abteilungen des Anorganischen Institutes für die stetige Unterstützung.

Ferner danke ich allen Mitarbeitern des Institutes für Physikalische Chemie der Universität Göttingen, die zum Gelingen dieser Arbeit beigetragen haben.

Allen meinen Freunden danke ich für die gelegentliche Ablenkung vom Studium, ohne euch wäre alles schwieriger und nur halb so schön gewesen.

Bei meiner Familie, besonders meinen Eltern möchte ich mich für ihre große Unterstützung während des gesamten Studiums ganz herzlich bedanken. 


\section{Lebenslauf}

\author{
Name \\ Geburtsdatum \\ Geburtsort \\ Familienstand \\ Nationalität
}

\section{Schulausbildung}

\author{
$1982-1989$ \\ 1989 - 1990 \\ $1990-1991$ \\ $1991-1998$ \\ Juli 1998
}

\author{
Duc Hung Nguyen \\ 14.April.1976 \\ Saigon/Vietnam \\ ledig \\ vietnamesisch und deutsch
}

\section{Hochschulstudium}

Oktober 1998

Oktober 2000

05/02 - 08/02

$12 / 02-05 / 03$

04/03-07/03

Juli 2003

seit Juli 2003

seit Januar 2004

seit Juli 2005

02/06-03/06
Besuch der 1. bis 7. Klasse in Saigon/Vietnam

Teilnahme am Unterricht der Vorbereitungsklasse für ausländische Schüler, Norden/Deutschland

Orientierungsstufe, Norden

Gymnasium Josephinum, Hildesheim

Erwerb der Allgemeinen Hochschulreife

Beginn des Chemiestudiums an der Georg-August-Universität Göttingen

Vordiplom in Chemie mit der Gesamtnote „Sehr gut“

Praktikum bei Bayer AG in Leverkusen zum Thema „Bestimmung von SADT (Self Accelerating Decomposition Temperature) mit Hilfe der Differentialthermoanalyse“

Diplomarbeit unter Anleitung von Prof. Dr. M. Buback zum Thema „Fluidphasen-Umsetzungen bei schneller Aufheizung mit überkritischem $\mathrm{CO}_{2}$ "

Studentische Hilfskraft am Institut für Physikalische Chemie der Universität Göttingen

Diplom in Chemie mit der Gesamtnote „Sehr gut"

Wissenschaftlicher Mitarbeiter des Instituts für Physikalische Chemie der Universität Göttingen

Promotion im Arbeitskreis von Prof. Dr. M. Buback Lehrstuhl am Institut für Technische und Makromolekulare Chemie der Universität Göttingen - zum Thema „RAFT-Polymerisation an Oberflächen“ unter Betreuung von Dr. P. Vana

Stipendiat des Graduiertenkollegs (GRK 782) „Spektroskopie und Dynamik molekularer Knäuel und Aggregate“ Forschungsaufenthalt an der Universität Leeds, England 